EPA/NMED/LANL 1998 Water Quality

Results: Statistical Analysis and

Comparison to Regulatory Standards

B. Gallaher

T. Mercier

P. Black

K. Mullen 


\section{DISCLAIMER}

This report was prepared as an account of work sponsored by an agency of the United States Government. Neither the United States Government nor any agency thereof, nor any of their employees, make any warranty, express or implied, or assumes any legal liability or responsibility for the accuracy, completeness, or usefulness of any information, apparatus, product, or process disclosed, or represents that its use would not infringe privately owned rights. Reference herein to any specific commercial product, process, or service by trade name, trademark, manufacturer, or otherwise does not necessarily constitute or imply its endorsement, recommendation, or favoring by the United States Government or any agency thereof. The views and opinions of authors expressed herein do not necessarily state or reflect those of the United States Government or any agency thereof. 


\section{DISCLAIMER}

Portions of this document may be illegible in electronic image products. Images are produced from the best available original document. 


\section{CONTENTS}

ABSTRACT.

1. INTRODUCTION

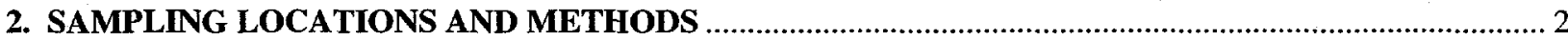

GROUNDWATER

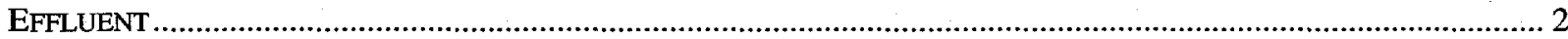

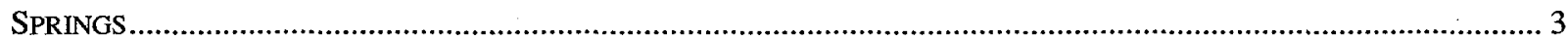

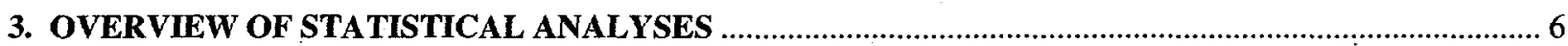

4. SUMMARY OF STATISTICAL TEST RESULTS

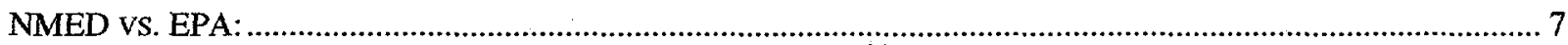

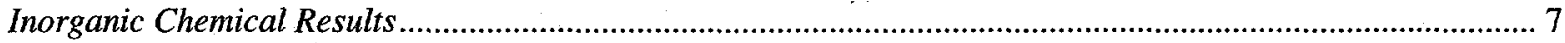

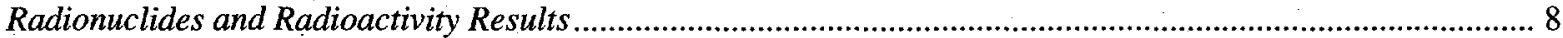

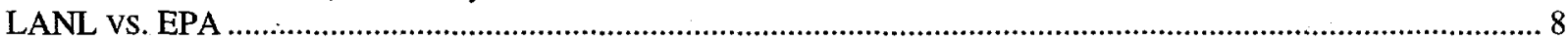

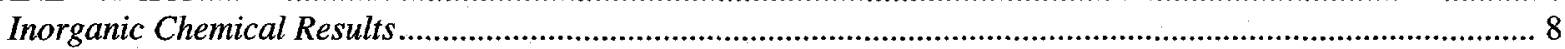

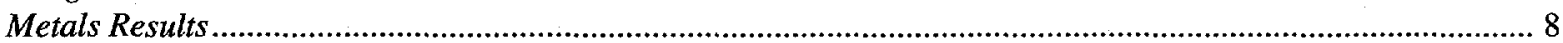

Radionuclides and Radioactivity Results ............................................................................................ 9

5. AGREEMENT IN COMPARISONS OF DATA FROM DIFFERENT SOURCES TO REGULATORY LIMITS

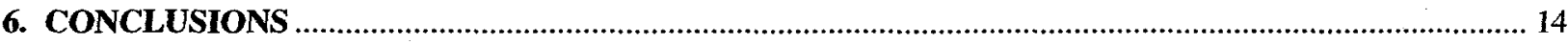

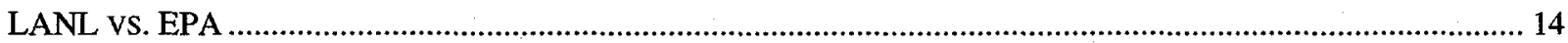

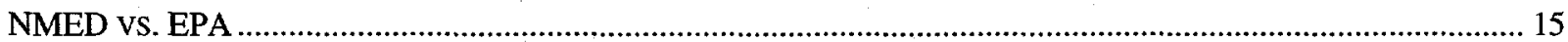

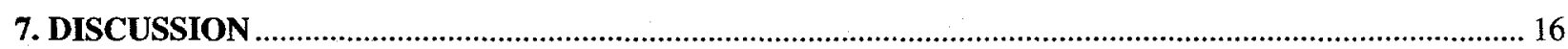

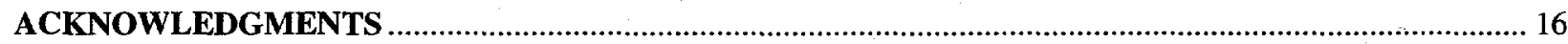

\section{APPENDIXES}

APPENDIX A - ASSUMPTIONS AND DATA SET CONVENTIONS USED IN REPORT ............................17

APPENDIX B - DATA AND RELATIVE PERCENT DIFFERENCES (RPDs) .............................................21

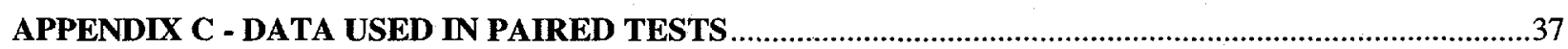

APPENDIX D - PLOTS OF DATA USED IN REGRESSION ANALYSES ...................................................47

APPENDIX E - GROUNDWATER QUALITY STANDARDS AND GUIDELINES …..................................57

APPENDIX F - COMPARISON OF DATA PAIRS AGAINST STANDARDS ……....................................61

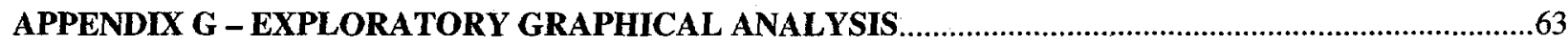




\title{
EPA/NMED/LANL 1998 Water Quality Results: Statistical Analysis and Comparison to Regulatory Standards
}

by

\author{
B. Gallaher, T. Mercier, P. Black, and K. Mullen
}

\begin{abstract}
Four governmental agencies conducted a round of groundwater, surface water, and spring water sampling at the Los Alamos National Laboratory during 1998. Samples were split among the four parties and sent to independent analytical laboratories. Results from three of the agencies were available for this study. Comparisons of analytical results that were paired by location and date were made between the various analytical laboratories. The results for over 50 split samples analyzed for inorganic chemicals, metals, and radionuclides were compared. Statistical analyses included non-parametric (sign test and signed-ranks test) and parametric (paired t-test and linear regression) methods. The data pairs were tested for statistically significant differences, defined by an observed significance level, or p-value, less than 0.05 . The main conclusion is that the laboratories' performances are similar across most of the analytes that were measured. In some $95 \%$ of the laboratory measurements there was agreement on whether contaminant levels exceeded regulatory limits. The most significant differences in performance were noted for the radioactive suite, particularly for gross alpha particle activity and Sr-90.
\end{abstract}

\section{INTRODUCTION}

During the autumn of 1998, the U.S. Environmental Protection Agency (EPA) led an extensive sampling of groundwater located within or adjacent to the Los Alamos National Laboratory (Laboratory, or LANL). In addition to the EPA, three other organizations participated in the sampling effort for added quality control purposes: the New Mexico Environment Department (NMED), the U.S. Geological Survey (USGS), and the Laboratory's Water Quality and Hydrology Group (ESH-18). Samples were split among the four parties and sent to independent laboratories. Samples of groundwater, effluent streams, and springs were collected and analyzed for a broad suite of potential contaminants, including radionuclides, metals, and synthetic organic chemicals. The EPA will use the sampling data to provide a "snapshot" of groundwater quality over a large portion of the watersheds draining the Laboratory and to evaluate the quality of the effluent discharges that may impact the groundwater quality within these watersheds.

The purpose of this study is to compare the analytical results from the various organizations participating in the sampling effort. [Results from the USGS were not available for inclusion in this statistical study.] Intra-laboratory comparisons of analytical results are an integral part of many organizations' quality control programs. Through such programs the general performance of an analytical laboratory may be assessed relative to other laboratories. Occasionally, an unrecognized analytical bias in a particular analytical laboratory's procedure may come to light when compared with results from other laboratories. 
For this study, over 1200 analytical results obtained from the sampling effort have been assembled and paired by sampling location and date. The pairs of results are then subject to a variety of statistical comparisons to determine if the laboratories' results are statistically different. For example, EPA's reported mercury concentration for a particular sampling location and date is paired and compared with NMED's or the Laboratory's mercury result obtained for the same sampling location and date. This process is repeated for all other related data pairs, resulting in multiple comparisons for mercury. The statistical tests provide an indication if there is an overall agreement between the various parties' results for a given chemical.

The key findings and study design are presented in the body of this report. Section 2 of this report discusses the field sampling protocols. Sampling locations and methods are summarized. Section 3 of this report presents an overview of the statistical analyses of the analytical results. Section 4 summarizes the results from the statistical analyses. Section 5 evaluates whether there is agreement between each organization's results when compared against regulatory limits. Section 6 of this report summarizes key conclusions of this investigation and discusses the significance of the study findings. The Appendices provide assumptions and conventions used in the data set preparation (Appendix A) and

- a tabulation of the data and relative percent differences between pairs (Appendix B),

- a tabulation of paired data statistical analyses (Appendix C),

- scatter plots of data used in regression analysis (Appendix D),

- groundwater standards and guidelines and comparisons to these guidelines (Appendixes $\mathrm{E}$ and F), and

- exploratory data presentations (Appendix G).

\section{SAMPLING LOCATIONS AND METHODS}

Sampling began in the middle of August and concluded toward the end of September 1998. The EPA and the NMED selected the sample locations, shown in Figures 1 and 2. Sampling was conducted both on the Laboratory and on adjacent San Ildefonso Pueblo lands. As a group, considerable emphasis was on sampling the shallow perched groundwater found in the floors of Los Alamos and Mortandad Canyons. A review of prior annual sampling results from the Laboratory's Environmental Surveillance Program helped the EPA and NMED target these sampling locations. A summary of the sample locations follows:

\begin{tabular}{ll}
\multicolumn{1}{c}{ Groundwater } \\
- & Mortandad Canyon (12 locations) \\
- & Los Alamos Canyon (11 locations) \\
- & Pueblo Canyon (2 locations) \\
San Ildefonso Pueblo Land (1 location) \\
$\quad$ Effluent \\
TA-50 Radioactive Liquid Waste Treatment Facility \\
- Outfall
\end{tabular}


- Bayo Canyon Sanitary Wastewater Treatment Plant

- Outfall

- Intersection between stream channel and State Route 502

- DP Canyon

\section{Springs}

- DP Spring

- $\quad$ San Ildefonso Pueblo Land

- Basalt Spring

- LA Spring

- La Mesita Spring

- Otowi Spring

- Sacred Spring

EPA contractor (TechLaw) personnel sampled all of the above locations. After an initial inspection of the condition of a well, water level and well depth measurements were taken. All but three of the wells were sampled using dedicated pumps (Teflon ${ }^{\mathrm{TM}}$ bladder sampling pumps for shallow wells or impeller pumps for wells deeper than 100 feet). Portable peristaltic pumps were used with new sampling tubing at the remainder of the wells. Each well usually was pumped for 15 to 30 minutes before sampling commenced to remove any stagnant water in the well casing. (The equivalent of 3 well casing volumes of water was targeted for purging from a well before sampling.) During the purge cycle, field measurements of turbidity, $\mathrm{pH}$, temperature, and specific electrical conductance were performed to assess the general water quality conditions at the well site.

Springs and effluent streams were usually sampled by lowering the intake of the peristaltic pump tubing into the streamflow and pumping the sample into appropriate pre-cleaned sample containers. Filtered samples were collected in pre-cleaned containers and then transferred to the appropriate sample containers using a peristaltic pump with a disposable in-line 0.45 -micron filter. All sample containers were labeled with unique blind alphanumeric identification codes and transferred to the appropriate analytical laboratory using full chain-of-custody procedures. EPA and NMED samples were shipped to contract commercial analytical laboratories. USGS samples were shipped to its internal National Water Quality Center. Samples collected for the Laboratory by ESH-18 personnel were submitted to internal analytical laboratory groups within the Chemical Science and Technology (CST) Division. 


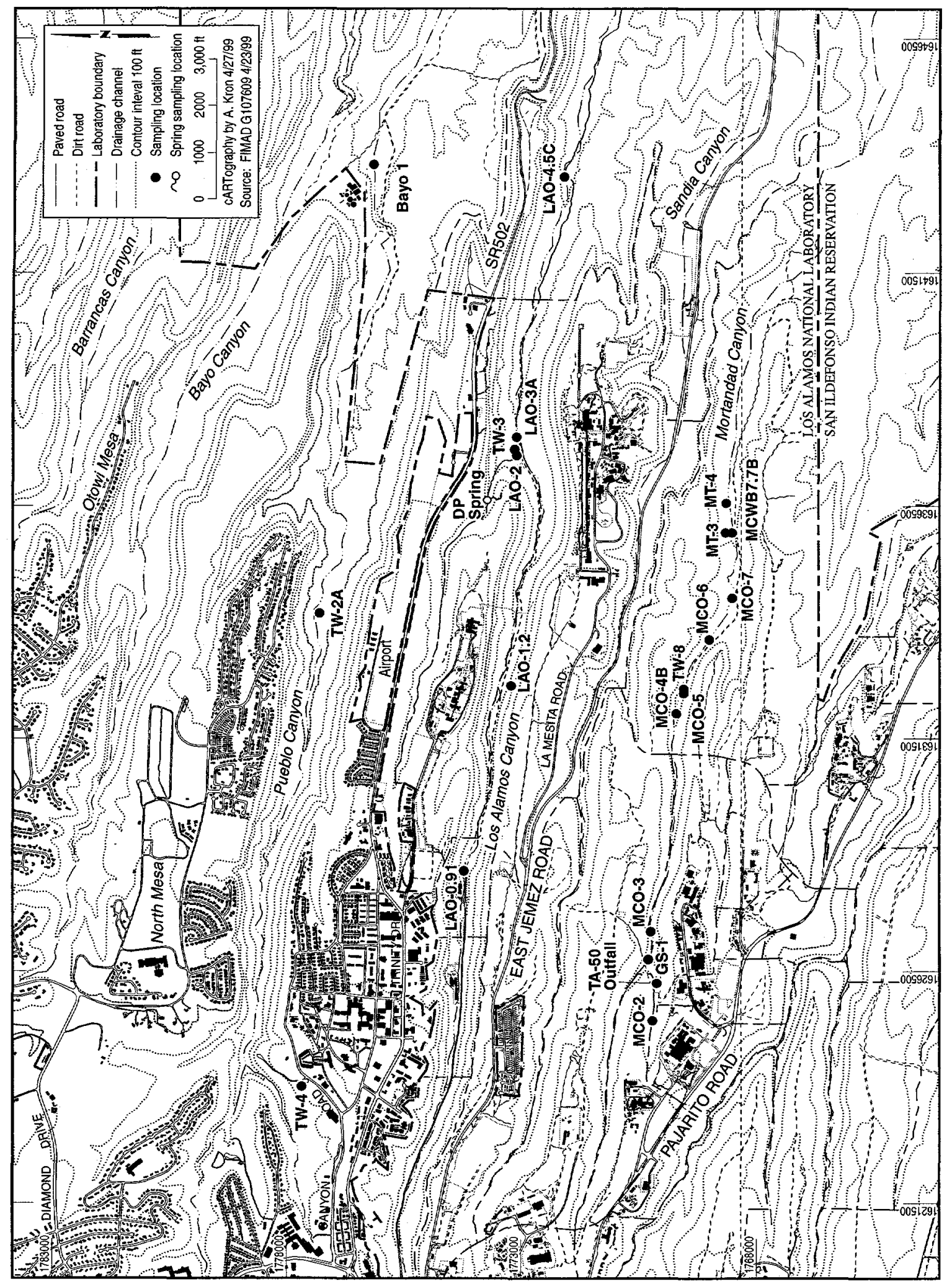

Figure 1. Western locations of sampling sites. 


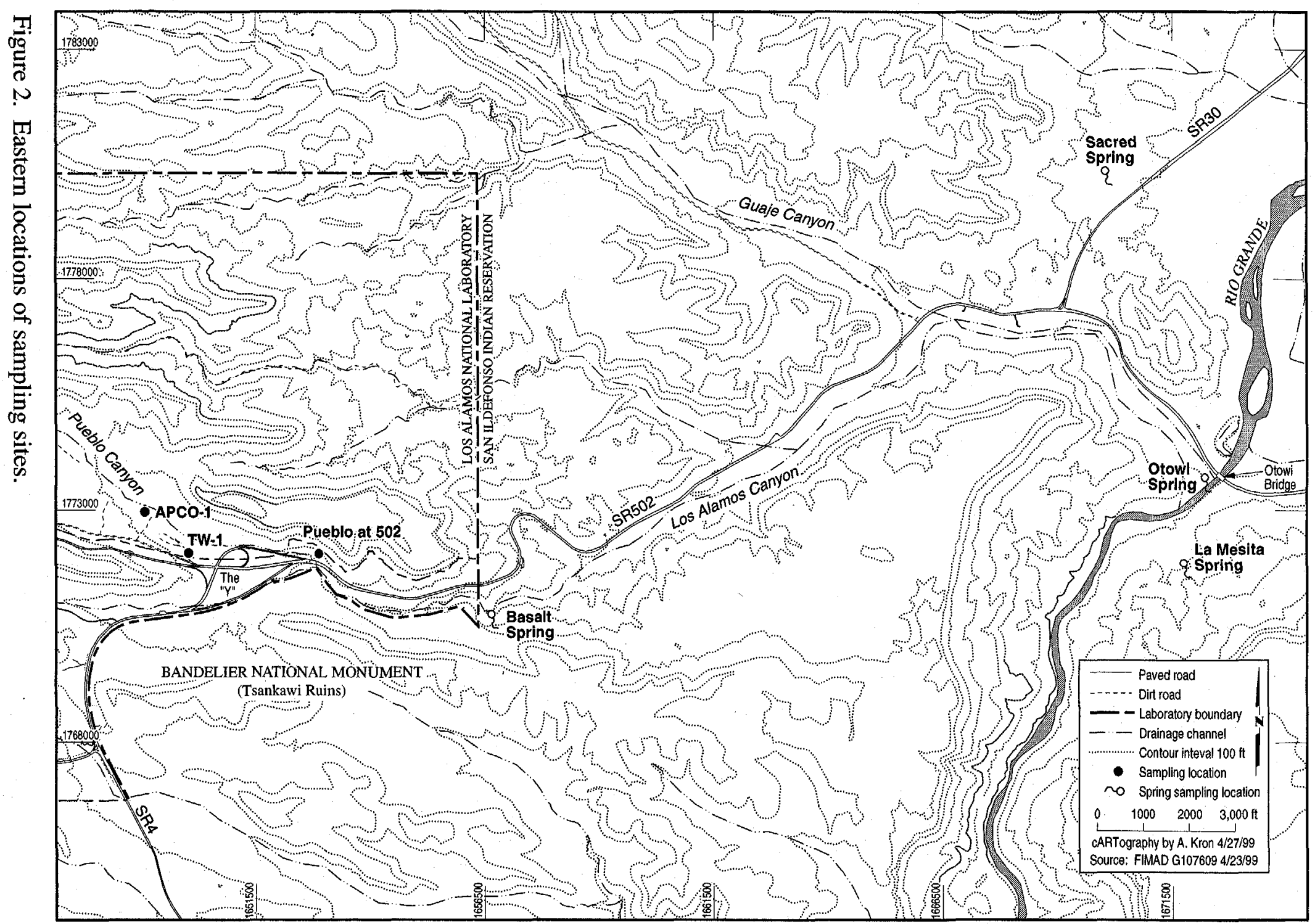




\section{OVERVIEW OF STATISTICAL ANALYSES}

Comparisons of analytical results were made between NMED and EPA, LANL and EPA, and LANL and NMED. Results were paired by location and sampling date'. (If two parties sampled a location on different days, the results were still paired ${ }^{2}$.) Prior to performing statistical tests, data tables and summaries of the data were prepared to gain an understanding of, and insights into, the data. The data summaries, including the calculations of relative percent differences, provided the first indications of potential differences in laboratory analytical performance.

These summaries are presented in Appendix B. Statistical analyses included non-parametric (sign test and signed-ranks test) and parametric (paired t-test and linear regression) statistical tests, which were used to verify differences that were observed in the data and data summaries. The phrase "significant" is equivalent to "statistically significant" in the following presentation. For all tests, "statistically significant" is defined by an observed significance level, or p-value, less than 0.05 .

The different statistical tests performed use different information contained in the data. The sign test considers only the sign of the differences between paired results (i.e., Does Lab 1 generally report higher results than Lab 2?). This test involves calculation of the ordered differences between paired data (Lab 1 minus Lab 2), followed by a count of the number of positive differences, and a statistical test to determine if this count is reasonable by random chance alone. Typically, the sign test will indicate a significant difference if there are substantially more positive differences than negative differences, or vice versa. The sign test also statistically evaluates the median difference.

Paired difference tests (paired t-test and signed-ranks test) consider the direction and magnitude of the differences between paired results (i.e., Is the average or median paired difference between Lab 1's results and Lab 2's results significantly different from zero?). The paired t-test involves calculation of the differences between paired data (Lab 1 minus Lab 2), calculation of the average paired difference, and a statistical test to determine if this average is large enough to suggest different performance of one laboratory compared to the other. The signed-ranks test involves ranking the absolute values of the differences between paired results and counting the number of ranks with positive signs. A statistical test determines if this sum is small enough to indicate a median difference unequal to zero. A paired t-test is run unless the assumption of normality is violated, in which case, a signed-ranks test is run. Data used in the paired difference tests are presented in Appendix C.

Regression analysis provides estimates of parameters describing the nature of the relationship between paired results. The regression evaluates the correlation between the paired data sets. The regression results are interpreted by considering the y-intercept and slope of the line fitted to plotted pairs of results. If paired labs produced identical results, the y-intercept would be indistinguishable from the origin $(x=0, y=0)$ and the slope would be indistinguishable from one. Plots of paired data used in the regression analysis are presented in Appendix D.

\footnotetext{
1 Note that data could not be grouped effectively across all three organizations because many samples were analyzed by only two organizations.

2 The statistical tests performed require paired data and certain independence assumptions that are violated when including paired samples collected on different dates. The effect of this violation is expected to be small because sampling protocols were similar and the sample data are expected to be reasonably homogeneous. However, an evaluation of the effect of this violation of underlying assumptions has not been performed.
} 
Analyses were not run unless there were at least five pairs of detected results. Thus, analytes that were rarely detected are not considered in this study. These include ammonia, total Kjeldahl nitrogen, arsenic, beryllium, chromium, copper, mercury, lead, and selenium. Analytes not included in the analyses due to lack of sufficient paired data include calcium, potassium, magnesium, sodium, strontium, uranium, organics, $\mathrm{Am}-241, \mathrm{Pu}-238$, and $\mathrm{Pu}-239$. There were insufficient paired results for any comparisons to be made between NMED and LANL. Paired comparisons were possible for NMED vs. EPA and LANL vs. EPA. To date, results from the USGS have not been received and so the following assessment involves data from the remaining three parties only.

\section{SUMMARY OF STATISTICAL TEST RESULTS}

Statistical test results are tabulated in Table 1. Shown are the results of the various statistical comparisons for those analytes with 5 or more pairs of detected results. For the NMED vs. EPA comparisons, 10 analytes were evaluated. For the LANL vs. EPA comparisons, 23 analytes were evaluated.

Test results indicating statistically significant differences are noted in bold in Table 1 . Tests that indicated no statistical difference are noted as "NSD" (no statistical difference). Discussion of the results for each analyte evaluated follows.

\section{NMED vs. EPA:}

\section{Inorganic Chemical Results}

$\mathrm{Cl}$ :

The sign test and paired t-test indicate that the median and average differences are not significantly different from zero. In addition the regression analysis indicates a significant relationship between paired results. The slope of the regression line is statistically less than one $(0.89)$, indicating that as EPA's results increase, NMED's increase at a lower rate.

F: $\quad$ The sign test and paired t-test indicate that the median and average differences are not significantly different from zero. Also, the regression analysis indicates a significant relationship between paired results. The intercept of the regression line is statistically greater than zero, indicating a slight high bias in the NMED results relative to EPA's.

Nitrate/Nitrite: The sign test indicates that the median difference is not significantly different from zero. The regression analysis indicates a significant relationship between paired results. The slope of the regression line is statistically less than one (0.81), indicating that as EPA's results increase, NMED's increase at a lower rate. This observation is supported by the result of the paired t-test that indicates that on average the difference (the NMED result minus the EPA result) is significantly less than zero.

SO4: The regression analysis indicates a significant relationship between paired results. The intercept of the regression line is statistically greater than zero and the slope is statistically less than one. This is supported by the sign test and the paired difference test, which indicate median differences not significantly different from zero.

Total Dissolved All tests indicate good agreement between NMED and EPA results. 

Radionuclides and Radioactivity Results
H-3:
All tests indicate good agreement between NMED and EPA results.
Sr-90:
While the sign test and paired t-test indicate that median and average differences are not significantly different from zero, the regression analysis
U-234: does not indicate a significant relationship between NMED and EPA results.
$\mathrm{U}-235$ : All tests indicate good agreement between NMED and EPA results.
While the sign test and paired $t$-test indicate that median and average differences are not significantly different from zero, the regression analysis does not indicate that there is a significant relationship between NMED and EPA results.
U-238: $\quad$ All tests indicate good agreement between NMED and EPA results.

\section{LANL vs. EPA}

\section{Inorganic Chemical Results}

Alkalinity:

$\mathrm{Cl}$ :

F:

Nitrate/Nitrite:

SO4:

TDS:

Total Suspended Solids (TSS):

Metals Results $\mathrm{Al}$ (filtered):

Al (unfiltered):

B (filtered):

B (unfiltered):
All tests indicate good agreement between LANL and EPA results. The sign test and the paired difference test indicate that the median differences are not significantly different from zero. Also, the regression analysis indicates a significant relationship between paired results. The slope of the regression line is statistically less than one $(0.84)$, indicating that as EPA's results increase, LANL's increase at a lower rate.

All tests indicate good agreement between LANL and EPA results. All tests indicate good agreement between LANL and EPA results. All tests indicate good agreement between LANL and EPA results. Although the sign test and paired t-test indicate that median and average differences are not significantly different from zero, the regression analysis does not indicate a significant relationship between LANL and EPA results. All tests indicate good agreement between LANL and EPA results.

Although the sign test and paired t-test indicate that median and average differences are not significantly different from zero, the regression analysis does not indicate that there is a significant relationship between LANL and EPA results.

Although the sign test and paired t-test indicate that median and average differences are not significantly different from zero, the regression analysis does not indicate that there is a significant relationship between LANL and EPA results. (Aluminum results can vary depending on the amount of suspended material in a sample and therefore the regression analysis result may be an indicator of split sample heterogeneity).

Although the regression analysis indicates good agreement between LANL and EPA results, the sign test and paired t-test indicate median and average differences that are significantly less than zero. This indicates that on average EPA is reporting higher values than LANL. This is reflected in the slightly negative $y$-intercept.

Although the regression analysis indicates good agreement between LANL and EPA results, the sign test and paired t-test indicate median and average 
$\mathrm{Ba}$ (filtered): $\quad$ All tests indicate good agreement between LANL and EPA results.

$\mathrm{Ba}$ (unfiltered): Although the regression analysis indicates good agreement between LANL and EPA results, the sign test and paired t-test indicate median and average differences that are significantly greater than zero. This indicates that on average LANL is reporting higher values than EPA. This is reflected in the slightly positive y-intercept.

Fe (filtered): $\quad$ Although the sign test and paired t-test do not indicate that median and average differences are significantly different from zero, the regression analysis does not indicate that there is a significant relationship between LANL and EPA results.

Fe (unfiltered): All tests indicate good agreement between LANL and EPA results. Mn (filtered): The sign test and paired t-test indicate that the median and average differences are not significantly different from zero. Also, the regression analysis indicates a significant relationship between paired results. The slope of the regression line is statistically greater than one (1.02), indicating that as EPA's results increase, LANL's increase at a slightly higher rate.

Mn (unfiltered): The sign test and paired difference test indicate that the median differences are not significantly different from zero. Also, the regression analysis indicates a significant relationship between paired results. The slope of the regression line is statistically greater than one (1.08), indicating that as EPA's results increase, LANL's increase at a slightly higher rate.

Mo (filtered): $\quad$ All tests indicate good agreement between LANL and EPA results.

Mo (unfiltered): All tests indicate good agreement between LANL and EPA results.

\section{Radionuclides and Radioactivity Results}

Gross Alpha: All tests indicate poor agreement between LANL and EPA results. The sign test and paired difference test indicate that EPA generally reports higher values.

Gross Beta: Although the sign test indicates that the median difference is significantly less than zero (EPA generally reports higher values), the paired difference test indicates that the median difference is not significantly different from zero. Also, the regression analysis indicates a significant relationship between paired results with estimates of the y-intercept and slope not statistically different from zero and one, respectively.

H-3: $\quad$ Although the regression analysis indicates good agreement between LANL and EPA results, the sign test and paired difference test indicate a median difference that is significantly greater than zero. This indicates that on average LANL is reporting higher values than EPA. This is reflected in the slightly positive $y$-intercept.

Sr-90: $\quad$ The sign test and paired difference test indicate median difference that is significantly greater than zero (LANL generally reports higher values than EPA). The regression analysis indicates that there is a significant relationship between LANL and EPA results. The estimate of the slope is statistically greater than one (1.64) indicating that as EPA's results increase, LANL's increase at a higher rate. This supports the conclusions of the sign test and paired t-test. 
Table 1. Summary of Statistical Test Results

\begin{tabular}{|c|c|c|c|c|c|c|c|c|c|c|c|}
\hline \multirow[t]{3}{*}{ ANALYTE* } & \multirow[t]{3}{*}{$\mathbf{F} / \mathbf{T}$} & \multicolumn{5}{|c|}{ NMED vs. EPA } & \multicolumn{5}{|c|}{ LANL vs. EPA } \\
\hline & & \multirow{2}{*}{$\begin{array}{l}\text { Sign Test } \\
\text { Median } \\
\text { Difference = } \\
\text { 0? }\end{array}$} & \multirow{2}{*}{$\begin{array}{l}\begin{array}{l}\text { Paired } \\
\text { Difference } \\
\text { Test }\end{array} \\
\text { Average or } \\
\text { Median } \\
\text { Difference } \\
=0 \text { ? }\end{array}$} & \multicolumn{3}{|c|}{ Regression } & \multirow{2}{*}{$\begin{array}{l}\text { Sign Test } \\
\text { Median } \\
\text { Difference = } \\
0 ?\end{array}$} & \multirow{2}{*}{\begin{tabular}{|l|}
$\begin{array}{l}\text { Paired } \\
\text { Diff. Test }\end{array}$ \\
Average or \\
Median \\
Difference \\
$=0 ?$ \\
\end{tabular}} & \multicolumn{3}{|c|}{ Regression } \\
\hline & & & & Significant? & $\begin{array}{l}\text { Intercept }= \\
0 \text { ? }\end{array}$ & $\begin{array}{l}\text { Slope }= \\
1 ?\end{array}$ & & & Significant? & $\begin{array}{l}\text { Intercept = } \\
0 ?\end{array}$ & $\begin{array}{l}\text { Slope }= \\
1 ?\end{array}$ \\
\hline \multicolumn{12}{|l|}{ Inorganics } \\
\hline Alkalinity & & & & & & & NSD & NSD & Yes & \begin{tabular}{|l|} 
NSD \\
4856 \\
\end{tabular} & \begin{tabular}{|l|} 
NSD \\
0.84
\end{tabular} \\
\hline $\mathrm{Cl}$ & & NSD & NSD & Yes & $\begin{array}{l}\text { NSD } \\
1305 \\
\end{array}$ & $\begin{array}{l}\text { No } \\
0.89\end{array}$ & NSD & NSD & Yes & \begin{tabular}{|l|} 
NSD \\
1771 \\
\end{tabular} & \begin{tabular}{|l|} 
No \\
0.84 \\
\end{tabular} \\
\hline $\bar{F}$ & & NSD & NSD & Yes & $\begin{array}{l}\text { No } \\
553 \\
\end{array}$ & $\begin{array}{l}\text { NSD } \\
0.69 \\
\end{array}$ & NSD & NSD & Yes & $\begin{array}{l}\text { NSD } \\
-64.9\end{array}$ & \begin{tabular}{|l|} 
NSD \\
1.28 \\
\end{tabular} \\
\hline Nitrate/Nitrite & & NSD & $\begin{array}{l}\text { No } \\
\text { EPA> } \\
\text { NMED } \\
\end{array}$ & Yes & \begin{tabular}{|l|} 
NSD \\
189
\end{tabular} & $\begin{array}{l}\text { No } \\
0.81\end{array}$ & NSD & NSD & Yes & \begin{tabular}{|l|} 
NSD \\
-18.6
\end{tabular} & $\begin{array}{l}\text { NSD } \\
0.95\end{array}$ \\
\hline $\mathrm{SO} 4$ & & NSD & NSD & Yes & \begin{tabular}{|l|} 
No \\
$\mathbf{3 0 3 4}$ \\
\end{tabular} & $\begin{array}{l}\text { No } \\
0.76\end{array}$ & NSD & NSD & Yes & \begin{tabular}{|l|} 
NSD \\
855 \\
\end{tabular} & $\begin{array}{l}\text { NSD } \\
0.89 \\
\end{array}$ \\
\hline TDS & & NSD & NSD & Yes & $\begin{array}{l}\text { NSD } \\
-96361\end{array}$ & $\begin{array}{l}\text { NSD } \\
1.21 \\
\end{array}$ & NSD & NSD & No & & \\
\hline TSS & & & & & & & NSD & NSD & Yes & $\begin{array}{l}\text { NSD } \\
-459\end{array}$ & $\begin{array}{l}\text { NSD } \\
0.84\end{array}$ \\
\hline
\end{tabular}


Table 1 (cont'd)

\begin{tabular}{|c|c|c|c|c|c|c|c|c|c|c|c|}
\hline \multirow[t]{3}{*}{ ANALYTE } & \multirow[t]{3}{*}{$\mathbf{F} / \mathbf{T}$} & \multicolumn{5}{|c|}{ NMED vs. EPA } & \multicolumn{5}{|c|}{ LANL vs. EPA } \\
\hline & & \multirow{2}{*}{$\begin{array}{l}\text { Sign Test } \\
\text { Median } \\
\text { Difference = } \\
0 ?\end{array}$} & \multirow{2}{*}{$\begin{array}{l}\text { Paired } \\
\text { Difference } \\
\text { Test } \\
\text { Average or } \\
\text { Median } \\
\text { Difference } \\
=0 ?\end{array}$} & \multicolumn{3}{|c|}{ Regression } & \multirow{2}{*}{$\begin{array}{l}\text { Sign Test } \\
\text { Median } \\
\text { Difference = } \\
0 ?\end{array}$} & \multirow{2}{*}{$\begin{array}{l}\text { Paired } \\
\text { Difference } \\
\text { Test } \\
\text { Average or } \\
\text { Median } \\
\text { Difference } \\
=0 ?\end{array}$} & \multicolumn{3}{|c|}{ Regression } \\
\hline & & & & Significant? & $\begin{array}{l}\text { Intercept = } \\
0 ?\end{array}$ & $\begin{array}{l}\text { Slope = } \\
1 ?\end{array}$ & & & Significant? & $\begin{array}{l}\text { Intercept }= \\
0 ?\end{array}$ & $\begin{array}{l}\text { Slope = } \\
1 ?\end{array}$ \\
\hline Metals & & & & & & & & & & & \\
\hline $\mathrm{Al}$ & $\mathrm{F}$ & & & & & & NSD & NSD & No & & \\
\hline & $\mathrm{T}$ & & & & & & NSD & NSD & No & & \\
\hline $\bar{B}$ & $\mathrm{~F}$ & & & $\therefore$ & & & $\begin{array}{l}\text { No } \\
\text { EPA }>\text { LANL }\end{array}$ & $\begin{array}{l}\text { No } \\
\text { EPA }>\text { LANL }\end{array}$ & Yes & $\begin{array}{l}\text { NSD } \\
-24.0 \\
\end{array}$ & $\begin{array}{l}\text { NSD } \\
0.92\end{array}$ \\
\hline & $\mathrm{T}$ & & & & & & $\begin{array}{l}\text { No } \\
\text { EPA }>\text { LANL }\end{array}$ & $\begin{array}{l}\text { No } \\
\text { EPA }>\text { LANL }\end{array}$ & Yes & $\begin{array}{l}\text { NSD } \\
-9.83 \\
\end{array}$ & $\begin{array}{l}\text { NSD } \\
0.89 \\
\end{array}$ \\
\hline$\overline{\mathrm{Ba}}$ & F & & & & & & NSD & NSD & Yes & $\begin{array}{l}\text { NSD } \\
0.88 \\
\end{array}$ & $\begin{array}{l}\text { NSD } \\
1.01\end{array}$ \\
\hline & $\mathrm{T}$ & & & & & & $\begin{array}{l}\text { No } \\
\text { LANL }>\text { EPA }\end{array}$ & $\begin{array}{l}\text { No } \\
\text { LANL }>\text { EPA }\end{array}$ & Yes & $\begin{array}{l}\text { NSD } \\
3.97 \\
\end{array}$ & $\begin{array}{l}\text { NSD } \\
1.00 \\
\end{array}$ \\
\hline $\mathrm{Fe}$ & $\mathrm{F}$ & & & & & & NSD & NSD & No & & \\
\hline 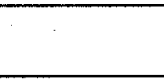 & $\mathrm{T}$ & & & & & & NSD & NSD & Yes & $\begin{array}{l}\text { NSD } \\
279 \\
\end{array}$ & $\begin{array}{l}\text { NSD } \\
0.99 \\
\end{array}$ \\
\hline $\mathrm{Mn}$ & $\mathrm{F}$ & & & & & & NSD & NSD & Yes & $\begin{array}{l}\text { NSD } \\
2.63\end{array}$ & $\begin{array}{l}\text { No } \\
1.02\end{array}$ \\
\hline & $T$ & & & & & & NSD & NSD & Yes & $\begin{array}{l}\text { NSD } \\
-0.07 \\
\end{array}$ & $\begin{array}{l}\text { No } \\
1.08 \\
\end{array}$ \\
\hline Mo & $F$ & & & & & & NSD & NSD & Yes & $\begin{array}{l}\text { NSD } \\
5.75 \\
\end{array}$ & $\begin{array}{l}\text { NSD } \\
1.01 \\
\end{array}$ \\
\hline & $\mathrm{T}$ & & & & & & NSD & NSD & Yes & $\begin{array}{l}\text { NSD } \\
-1.26 \\
\end{array}$ & $\begin{array}{l}\text { NSD } \\
1.07 \\
\end{array}$ \\
\hline
\end{tabular}


Table 1 (cont'd)

\begin{tabular}{|c|c|c|c|c|c|c|c|c|c|c|c|}
\hline \multirow[t]{3}{*}{ ANALYTE } & \multirow{3}{*}{$\mathbf{F} / \mathbf{T}$} & \multicolumn{5}{|c|}{ NMED vs. EPA } & \multicolumn{5}{|c|}{ LANL vs. EPA } \\
\hline & & \multirow{2}{*}{$\begin{array}{l}\text { Sign Test } \\
\text { Median } \\
\text { Difference = } \\
\text { 0? }\end{array}$} & \multirow{2}{*}{$\begin{array}{l}\text { Paired } \\
\text { Difference } \\
\text { Test } \\
\text { Average or } \\
\text { Median } \\
\text { Difference } \\
=0 ?\end{array}$} & \multicolumn{3}{|c|}{ Regression } & \multirow{2}{*}{$\begin{array}{l}\text { Sign Test } \\
\text { Median } \\
\text { Difference = } \\
\text { 0? }\end{array}$} & \multirow{2}{*}{$\begin{array}{l}\text { Paired } \\
\text { Difference } \\
\text { Test } \\
\text { Average or } \\
\text { Median } \\
\text { Difference } \\
=0 ?\end{array}$} & \multicolumn{3}{|c|}{ Regression } \\
\hline & & & & Significant? & $\begin{array}{l}\text { Intercept }= \\
0 ?\end{array}$ & $\begin{array}{l}\text { Slope = } \\
1 \text { ? }\end{array}$ & & & Significant? & $\begin{array}{l}\text { Intercept }= \\
0 ?\end{array}$ & $\begin{array}{l}\text { Slope = } \\
1 ?\end{array}$ \\
\hline \multicolumn{12}{|l|}{ Rads } \\
\hline Gross Alpha & & & & & & & $\begin{array}{l}\text { No } \\
\text { EPA }>\text { LANL }\end{array}$ & $\begin{array}{l}\text { No } \\
\text { EPA }>\text { LANL }\end{array}$ & No & & \\
\hline Gross Beta & & & . & & & & $\begin{array}{l}\text { No } \\
\text { EPA }>\text { LANL } \\
\end{array}$ & NSD & Yes & \begin{tabular}{|l|} 
NSD \\
-6.16 \\
\end{tabular} & $\begin{array}{l}\text { NSD } \\
1.29 \\
\end{array}$ \\
\hline $\mathrm{H}-3$ & & NSD & NSD & Yes & $\begin{array}{l}\text { NSD } \\
-90.8\end{array}$ & $\begin{array}{l}\text { NSD } \\
1.02\end{array}$ & $\begin{array}{l}\text { No } \\
\text { LANL>EPA }\end{array}$ & $\begin{array}{l}\text { No } \\
\text { LANL>EPA }\end{array}$ & Yes & $\begin{array}{l}\text { NSD } \\
483\end{array}$ & $\begin{array}{l}\text { NSD } \\
1.01\end{array}$ \\
\hline $\mathrm{Sr}-90$ & & NSD & NSD & No & & & $\begin{array}{l}\text { No } \\
\text { LANL>EPA }\end{array}$ & $\begin{array}{l}\text { No } \\
\text { LANL>EPA }\end{array}$ & Yes & $\begin{array}{l}\text { NSD } \\
0.81\end{array}$ & $\begin{array}{l}\text { No } \\
1.64\end{array}$ \\
\hline U-234 & & NSD & NSD & Yes & $\begin{array}{l}\text { NSD } \\
0.06 \\
\end{array}$ & $\begin{array}{l}\text { NSD } \\
0.85 \\
\end{array}$ & & & & & \\
\hline U-235 & & NSD & NSD & No & & & & & & & \\
\hline U-238 & & NSD & NSD & Yes & $\begin{array}{l}\text { NSD } \\
-0.08 \\
\end{array}$ & $\begin{array}{l}\text { NSD } \\
1.07 \\
\end{array}$ & & & & & \\
\hline
\end{tabular}

Analyte:

Blank entry:

F/T:

NMED vs. EPA

LANL vs. EPA:

Sign Test:

Median Difference $=0$ ?

Paired Difference Test:

Regression:

\section{Significant?}

Intercept $=0$ ?

Slope $=1$ ?
*Explanation of Column Headers:

Analyte under consideration

Insufficient number of data pairs to statistically evaluate (i.e., $<5$ data pairs)

$\mathrm{F}=$ Filtered; $\mathrm{T}=$ Unfiltered

Comparison of NMED result (Lab 1) and EPA result (Lab 2)

Comparison of LANL result (Lab 1) and EPA result (Lab 2)

Is the median difference (Lab 1 minus Lab 2$)$ statistically significantly different from zero? $(p=0.05)$

Is the median difference (Lab 1 minus Lab 2) statistically

NSD: Not statistically significantly differ
Statistically significant results bolded

Is the average or median difference (Lab 1 minus Lab 2$)$ statistically significantly different from zero? $(p=0.05)$

NSD: Not statistically significantly different from zero

Statistically significant results bolded

Summary of regression analysis

Is the relationship between paired Lab 1 and Lab 2 results statistically significant?

Is the intercept term statistically different from 0 ?

NSD: Not statistically significantly different from zero

Statistically significant results bolded

Is the slope term statistically different from 1 ?

NSD: Not statistically significantly different from one

Statistically significant results bolded 


\section{AGREEMENT IN COMPARISONS OF DATA FROM DIFFERENT SOURCES TO REGULATORY LIMITS}

An additional gauge of the analytical laboratories' performance is whether the same conclusion is reached about meeting regulatory limits, regardless of which laboratory performed the test. The issue concerns one of laboratory consistency in whether groundwater contamination is indicated (defined in this study as exceeding water quality standards).

Pairs of analytical results from NMED and EPA, LANL and EPA, and LANL and NMED were compared. Results were paired by location and sampling date. Each data pair was then compared against the most stringent groundwater quality standard or guideline for that particular analyte. (Does the Lab 1 result agree with Lab 2? Are the results concordant? That is, are both results above [or below] the regulatory limit?) Results for unfiltered and filtered water samples were pooled for this analysis. NMED's results for beryllium are not included in the comparisons because their laboratory's minimum analytical detection limit for this analyte is greater than the regulatory limit for that analyte. Selenium results are excluded because only LANL has a minimum analytical detection limit at or below the regulatory limit, and, thus, there were no results for comparison. Groundwater quality standards and guidelines set by the U.S. EPA, the State of New Mexico, and the U.S. Department of Energy are tabulated in Appendix E.

Table 2 summarizes the outcome of this comparison. There was total agreement between the NMED and LANL results and high agreement between NMED and EPA (96\%) and between LANL and EPA (94\%). In total, of the 597 pairs of data compared, approximately 95 percent of the results were concordant when compared against the regulatory standards. The table is a summation of comparisons of data pairs against the minimum groundwater quality standards and guidelines listed in Appendix E. Concordant pairs occur when both results are either above or below the respective regulatory standards.

Table 2. Agreement between Analytical Laboratories' Results when Compared Against Regulatory Limits.

\begin{tabular}{|l|c|c|c|}
\hline & NMED vs. EPA & NMED vs. LANL & LANL vs. EPA \\
\hline Number of data pairs evaluated & 143 & 70 & 387 \\
\hline $\begin{array}{l}\text { Number of concordant pairs above } \\
\text { or below regulatory limit }\end{array}$ & 139 & 68 & 363 \\
& $(96 \%)$ & $(100 \%)$ & $(94 \%)$ \\
\hline
\end{tabular}

Of the 24 analytes evaluated, the laboratories agreed uniformly on all but eight (Appendix F). Table 3 lists the eight analytes with discordant data pairs not uniformly matching the other laboratory's result.

Of the 570 concordant pairs, $321(56 \%)$ are for analytes whose values never exceed the regulatory standard: chloride, sulfate, arsenic, boron, barium, beryllium, chromium, copper, mercury, molybdenum, and tritium. In most cases, the regulatory standard far exceeds the observed value. For example, referring to the LANL vs. EPA scatter plot for barium (see Appendix D), the maximum value plotted is $147 \mu \mathrm{g} / \mathrm{L}$ while the regulatory standard is almost an order of magnitude higher at $1000 \mu \mathrm{g} / \mathrm{L}$. 
Table 3. Analytes with Discordant Data Pairs.*

\begin{tabular}{|c|c|c|c|}
\hline & NMED vs. EPA & $\begin{array}{c}\text { NMED vs. } \\
\text { LANL }\end{array}$ & LANL vs. EPA \\
\hline $\mathrm{F}$ & $1 / 7$ & & \\
\hline $\mathrm{TDS}$ & & & $2 / 5$ \\
\hline $\mathrm{Al}$ & $1 / 10$ & $\mathbf{1 / 4}$ & $7 / 26$ \\
\hline $\mathrm{Fe}$ & $1 / 10$ & $1 / 5$ & $6 / 26$ \\
\hline $\mathrm{Mn}$ & & & $2 / 26$ \\
\hline $\mathrm{Pb}$ & $1 / 10$ & & $4 / 28$ \\
\hline Gross alpha & & & $2 / 14$ \\
\hline Sr-90 & $1 / 10$ & & $1 / 14$ \\
\hline
\end{tabular}

*Ratio shown = Number of data pairs not matching when compared against regulatory standards/Total number of data pairs evaluated for that analyte.

For all other analytes, those for which at least one result exceeds the regulatory standard, $89 \%$ of the pairs are concordant ( 249 of 279 ). The decrease in the percent of agreement between paired values is an indication that, as reported values approach the regulatory standard, more "disagreements" occur. Most of the discordant data pairs are aluminum, iron, and lead results. Examination of selected scatter plots in Appendix D helps to visualize the differences between these data pairs. Plots with vertical and horizontal reference lines are divided into four quadrants. Points falling into the lower left and upper right quadrants are concordant pairs, i.e., both values would lead to the same conclusion. Points plotted in the upper right quadrant indicate that both results would lead to the decision that a standard was exceeded, while those plotted in the lower left quadrant would indicate that both results were less than the standard. Points falling in the upper left and lower right quadrants indicate disagreement between results. Points plotted in the upper left quadrant indicate that while results reported by LANL would lead to the conclusion that a standard was exceeded, the paired results reported by EPA would lead to the opposite conclusion. The reverse situation occurs for points plotted in the lower right quadrant. For example, the plot comparing aluminum values reported by LANL and EPA reveals that there is poor agreement between paired results and that all discordant data pairs fall in the upper left quadrant, i.e., LANL reported values that exceeded the standard, while those reported by EPA did not.

\section{CONCLUSIONS}

The main conclusion is that the laboratories' performances are similar across most of the analytes that were measured. However, there are a few exceptions that warrant further investigation to understand why the differences occurred.

\section{LANL vs. EPA}

Overall, results of inorganics and metals analyses from LANL and EPA samples correspond nicely, but some differences should be noted.

- There is no statistically significant correlation between filtered aluminum results, unfiltered aluminum, or filtered iron results. An investigation of associated total dissolved solids and total suspended solids measurements may reveal that this discrepancy is due to a sampling 
problem resulting in sample split heterogeneity caused by differing amounts of suspended and dissolved solids.

- Sign test and paired t-test results for both filtered and unfiltered boron results indicate a slight high relative bias in EPA's results. The mean differences for filtered and unfiltered results are 35 and $23 \mu \mathrm{g} / \mathrm{L}$, respectively.

- Results of both the sign test and paired t-tests point to a tendency for LANL to report higher results for unfiltered barium. The mean difference is $3.9 \mu \mathrm{g} / \mathrm{L}$.

Generally, there is less agreement between LANL and EPA radionuclide results.

- All tests indicate that there is disagreement between LANL and EPA gross alpha measurements. The median difference between $\mathrm{LANL}$ and $\mathrm{EPA}$ is $-4.1 \mathrm{pCi} / \mathrm{L}$, indicating the EPA generally reports higher results.

- Statistical tests also consistently point to a disparity between LANL and EPA Sr-90 results. The median difference between LANL and EPA is $0.63 \mathrm{pCi} / \mathrm{L}$, indicating that LANL generally reports higher results.

- A similar situation occurs for tritium. Generally, LANL reports higher results than EPA. The median difference is $240 \mathrm{pCi} / \mathrm{L}$.

\section{NMED vS. EPA}

Results of inorganic analysis from NMED and EPA generally seem to agree.

There seems to be less agreement between rad results reported by NMED and EPA. This is similar to the results reported above for LANL and EPA.

- There is no statistically significant correlation between paired Sr-90 results or between paired U-235 results reported from these two organizations. Lack of a statistical relationship between paired Sr-90 results is due in part to an outlier data point at the high end of the reported range of values.

Although there are statistically significant correlations between paired U-234 and paired U-238 results reported by NMED and EPA, both seem to be generated by one extreme value at the high range of reported results. This may mean that there is agreement between the labs at high activity levels, but less agreement at lower levels. U-234, U-235, and U-238 usually exist in equilibrium. Therefore, if a relationship between results exists for one isotope, there should be relationships between results for all isotopes. Since none exists for U-235, the relationships for U-234 and U-238 are called into question.

Some other statistical differences were observed, but those reported in this section appear to be the most significant, and exhibit general agreement across the statistical tests. The remaining sporadic statistically significant results do not provide evidence of a difference between paired results as strong as those presented above, and some such significant results can be expected considering the large number of statistical tests performed on the same data set. Approximately 100 tests were performed, of which $5 \%$ or so can be expected to indicate a significant result based on chance alone. 
There is generally good agreement between paired results in the context of meeting regulatory standards. The regulatory standards for analytes with many of these concordant pairs far exceed the maximum reported value. For those analytes where the values bracket the standard, the rate of agreement is somewhat lower. The analytes with the poorest rate of agreement are those that tend to be highly influenced by sample collection procedures.

\section{DISCUSSION}

This study was intended to quantitatively compare the results from the various analytical laboratories. A variety of statistical tools were selected to efficiently diagnose significant differences in the analytical results. Although the differences noted in some of the data results are statistically significant, the differences are not likely to be important from a water quality assessment perspective, the intended purpose of EPA's survey. In other words, the same general conclusions regarding contamination probably would be indicated by each of the laboratories' results. At locations where water quality standards or limits are exceeded or approached, the data from any of the analytical laboratories would allow for identification of the contamination problem.

A caveat to the above paragraph comes in the area of water quality detection monitoring, where investigators are looking for subtle, gradual upward trends in contaminant concentrations. In such cases, the investigators should be keenly aware of the inherent uncertainties in analytical results at low concentrations. To minimize the uncertainty, routine use of the same analytical laboratory may be helpful, assuming that its quality control performance is acceptable.

\section{ACKNOWLEDGMENTS}

Funding for the Laboratory's split sampling and statistical analyses came from the Environmental Surveillance Program. We would like to thank Max Maes and Penny Gomez of the Laboratory's Water Quality and Hydrology Group (ESH-18) for their expert field assistance during the sampling phase. Bruce Gallaher and Ken Mullen (both ESH-18) provided the technical liaison with the other organizations and the comparison with regulatory limits. Terre Mercier and Paul Black of Neptune and Co., Inc., performed the statistical analyses for ESH-18. 


\section{APPENDIX A ASSUMPTIONS AND DATA SET CONVENTIONS USED IN REPORT}

To allow for the computerized analyses performed in this study, some standardization of the data set was performed. For example, in several cases, the names of the sample stations or analytes differed slightly from those used by the other participating organizations. The following tabulation presents the assumed set of equivalents and standardized descriptors used in the analyses. It is presented to allow the EPA and NMED to track any changes to their original data records.

\section{ASSUMED LOCATION EQUIVALENTS}

A. BAYO-1 (OUTFALL):

1. BAYO SEWAGE OUTFALL

2. BAYO-1

3. BAYO-1(OUTFALL)

B. MORTANDAD AT GS-1

1. MORTANDAD @ GS-1

2. MORTANDAD @ GS-1 (GAGE E200)

3. MORTANDAD AT GS-1

C. PUEBLO AT SR-502

1. PUEBLO @ SR502 (GAGE 060)

2. PUEBLO AT SR-502

D. TA-50 OUTFALL

1. TA-50 OUTFALL

2. TA-502A

$3 . \quad$ TA-50-2A

E. TW-1

1. TEST WELL 1

2. TW-1

F. TW-2A

1. TEST WELL 2A

2. TW-2A

G. TW-3

1. TEST WELL 3

2. TW-3

H. TW -4

1. TEST WELL 4

2. TW-4

I. TW-8

1. TEST WELL 8

2. TW-8

J. LA MESITA SPRING

1. LA MESITA SP.

2. LA MESITA SPRING 


\section{ASSUMED ANALYTE EQUIVALENTS}

A. $\quad 540-59-0$

1. 1,2-DICHLOROETHENE

2. 540-59-0

B. ALKALINITY

1. TALK

2. ALKALINITY

C. GROSS ALPHA

1. ALPHA

2. GROSS ALPHA

D. GROSS BETA

1. BETA

2. GROSS BETA

E. $\quad \mathrm{CR}$

1. CHROMIUM

2. CR

F. $\quad \mathrm{CL}$

1. CL(-1)

2. CL

G. $\mathrm{F}$

1. $\quad F(-1)$

2. $\mathrm{F}$

H. NITRATE/NITRITE

1. $\mathrm{NO} 2 / \mathrm{NO} 3$ AS N

2. NITRATE/NITRITE

$3 . \quad \mathrm{NO} 3-\mathrm{N}$

I. PU-239

1. PU-239+240

2. PU-239

J. $\quad \mathrm{SO} 4$

1. $\mathrm{SO} 4(-2)$

2. $\mathrm{SO} 4$

K. TOT. KJELDAHL N

1. TKN

2. TOT. KJELDAHL N

L. U-235

1. URANIUM-235/236

2. U-235

M. URANIUM

1. U

2. URANIUM 


\section{GENERAL CONVENTIONS}

$<\#$ and \#U converted to

Symbol $=$ ' $<$ '

Result = \#

Results reported in $\mathrm{mg} / \mathrm{L}$ converted to $\mu \mathrm{g} / \mathrm{L}$.

Units: Radioactivity: $\quad \mathrm{pCi} / \mathrm{L}$

All other analytes: $\mu \mathrm{g} / \mathrm{L}$

Records uniquely identified (and matched) by:

Location

Sample type: Filtered/Unfiltered

Analyte

NOTE:Some sampling dates are different for some matched records.

\section{DATA SET SPECIFIC CONVENTIONS}

\section{NMED}

If there is no indication of sample type (Filtered/Unfiltered), it was assumed that the sample was unfiltered.

We used data even if it was qualified:

B:

Analyte found in blank.

LT:

Result is less than requested MDC, greater than sample specific MDC.

D: $\quad$ Compounds identified in an analysis at a secondary dilution factor.

§: $\quad$ Holding Times Broken.

\section{EPA}

If there is no indication of sample type (Filtered/Unfiltered), it was assumed that the sample was unfiltered.

It was assumed that sample type = 'UF' meant 'Unfiltered'

There are 23 records with no "date sampled" recorded. They were all associated with LAO-0.91. Some records for this station had "date sampled" recorded as 09/03/98. We have used all data for this station in the analysis, regardless of whether "date sampled" was recorded.

We used data even if it was qualified:

B or J 


\section{LANL}

We only used data with:

1. Date greater than or equal to $01 / 01 / 98$

2. Matrix not equal to:

a. 'QC' or

b. 'SED'

3. QC Type equal to:
a. 'CLIENT SAMPLE'
b. 'CUSTOMER SAMPLE'
c. 'SAMPLE'

Deleted "duplicates" as defined by records with identical location /analyte / $\mathrm{p}$ combinations:

1. 23 records for Am-241 where Techcode $=$ 'GENERIC GAMMA'. The records where Techcode = 'AM RAS ENV' were retained.

2. 139 records were deleted because they looked like lab duplicates. The Sample IDs differed by 1 character:
a. MM98051G5CM
MM98052G5CM
b. MF98051G5CM
MF98052G5CM
c. MF98081G3TM
MM98081G3TM
d. MM98081G8WT
MM98082G8WT

We have assumed that:

1. F: filtered

2. UF, UK (bolded and italicized in RPD printout), UUF and IF: unfiltered

We used data even if it was qualified:

B 


\section{APPENDIX B \\ DATA AND RELATIVE PERCENT DIFFERENCES (RPDs)}

This appendix tabulates all the data pairs assembled for this study and the calculated relative percent differences (RPDs). The RPDs are shown under the columns headers "NMED vs. EPA," "NMED vs. LANL," and "LANL vs. EPA." The RPDs are calculated according to the formula:

$$
\text { RPD }=((\text { Result from Lab } 1-\text { Result from Lab 2)/(Result from Lab } 1+\text { Result from Lab 2)/2) }) \times 100
$$

RPDs calculated are for all paired results, regardless of detection status.

RPDs calculated for pairs of detected results are noted by '*'.

Chemical concentrations are presented in the unit of $\mu \mathrm{g} / \mathrm{L}$ (micrograms per liter). Radionuclides or radioactivity are presented in the unit of $\mathrm{pCi} / \mathrm{L}$ (picocuries per liter).

The Table is organized by:
A. Sample Type:
1. Unfiltered
2. Filtered
B. Analytical suite
1. Inorganics
2. Metals
3. Organics
4. Rads (Radionuclides and Radioactivity)
C. Analyte
D. Location

We have assumed the following correspondence from the data files:

1. F:

2. UF, UK (bolded and italicized in RPD table), UUF and IF: unfiltered

\section{Explanation of Column Headers:}

Location:

NM Date:

EPA Date:

LANL Date:

NMED Result:

EPA Result:

LANL Result:

NMED vs. EPA:

NMED vs. LANL:

LANL vs. EPA:
Sampling location

NMED sampling date

EPA sampling date

ESH-18 sampling date

Result reported by NMED

Result reported by EPA

Result reported to ESH- 18 by LANL lab

Calculated RPD where NMED $=\mathrm{Lab} 1$ and $\mathrm{EPA}=\mathrm{Lab} 2$

Calculated RPD where NMED $=\mathrm{Lab} 1$ and $\mathrm{LANL}=\mathrm{Lab} 2$

Calculated RPD where $\mathrm{LANL}=\mathrm{Lab} 1$ and $\mathrm{EPA}=\mathrm{Lab} 2$ 


\begin{tabular}{|c|c|c|c|c|c|c|}
\hline Location & NM Date & EPA Date & $\begin{array}{l}\text { LANL } \\
\text { Date }\end{array}$ & $\begin{array}{l}\text { NMED } \\
\text { Result }\end{array}$ & $\begin{array}{l}\text { EPA } \\
\text { Result }\end{array}$ & $\begin{array}{l}\text { LANL } \\
\text { Result }\end{array}$ \\
\hline TW-1 & . & $09 / 01 / 98$ & $05 / 28 / 98$ & . & 111000.00 & 109000 \\
\hline TW-2A & . & $09 / 01 / 98$ & $09 / 01 / 98$ & . & 56600.00 & 57000 \\
\hline TW-3 & . & $09 / 01 / 98$ & $09 / 01 / 98$ & . & 103000.00 & 80000 \\
\hline TW-4 & . & $09 / 01 / 98$ & $09 / 01 / 98$ & . & 72700.00 & 64000 \\
\hline TW-8 & & $09 / 02 / 98$ & $09 / 02 / 98$ & & 74700.00 & 65000 \\
\hline
\end{tabular}

$\begin{array}{lll}\text { NMED vs } & \text { NMED vs } & \text { LANL vs } \\ \text { EPA } & \text { LANL } & \text { EPA } \\ . & . & -1.8182^{*} \\ . & . & 0.7042^{*} \\ . & . & -25.1366^{*} \\ . & . & -12.7286^{*} \\ . & . & -13.8869^{*}\end{array}$

SUITE $=$ Inorganics Analyte $=$ AMMONLA Unit $=\mu \mathrm{G} / \mathrm{L}$

\begin{tabular}{|c|c|c|c|c|c|c|c|c|c|}
\hline Location & NM Date & EPA Date & $\begin{array}{l}\text { LANL } \\
\text { Date }\end{array}$ & $\begin{array}{l}\text { NMED } \\
\text { Result }\end{array}$ & $\begin{array}{l}\text { EPA } \\
\text { Result }\end{array}$ & $\begin{array}{l}\text { LANL } \\
\text { Result }\end{array}$ & $\begin{array}{l}\text { NMED vs } \\
\text { EPA }\end{array}$ & $\begin{array}{l}\text { NMED vs } \\
\text { LANL }\end{array}$ & $\begin{array}{l}\text { LANL vs } \\
\text { EPA }\end{array}$ \\
\hline BAYO-1 (OUTFALL) & 09/01/98 & $09 / 01 / 98$ & . & 11000 & 11500 & . & $-4.444^{*}$ & . & . \\
\hline DP SPRING & 09/02/98 & $09 / 02 / 98$ & . & 60 & $<50$ & . & 18.182 & . & . \\
\hline $\mathrm{MCO}-5$ & $08 / 20 / 98$ & $08 / 20 / 98$ & . & $<500$ & $<50$ & . & 163.636 & . & . \\
\hline MCO-6 & $08 / 21 / 98$ & $08 / 21 / 98$ & . & $<500$ & $<50$ & . & 163.636 & . & . \\
\hline $\mathrm{MCO}-7$ & $08 / 21 / 98$ & $08 / 21 / 98$ & . & $<500$ & $<50$ & . & 163.636 & . & . \\
\hline TW-1 & $09 / 01 / 98$ & $09 / 01 / 98$ & . & 340 & $<50$ & . & 148.718 & . & . \\
\hline TW-8 & $09 / 02 / 98$ & $09 / 02 / 98$ & . & $<500$ & $<50$ & . & 163.636 & . & . \\
\hline
\end{tabular}

\begin{tabular}{|c|c|c|c|c|c|c|c|c|c|}
\hline Location & NM Date & EPA Date & $\begin{array}{l}\text { LANL } \\
\text { Date }\end{array}$ & $\begin{array}{l}\text { NMED } \\
\text { Result }\end{array}$ & $\begin{array}{l}\text { EPA } \\
\text { Result }\end{array}$ & $\begin{array}{l}\text { LANL } \\
\text { Result }\end{array}$ & $\begin{array}{l}\text { NMED vs } \\
\text { EPA }\end{array}$ & $\begin{array}{l}\text { NMED vs } \\
\text { LANL }\end{array}$ & $\begin{array}{l}\text { LANL vs } \\
\text { EPA }\end{array}$ \\
\hline BAYO-1 (OUTFALL) & $09 / 01 / 98$ & $09 / 01 / 98$ & . & 36400 & 39800.00 & . & $-8.9239 *$ & . & . \\
\hline DP SPRING & $09 / 02 / 98$ & $09 / 02 / 98$ & . & 25700 & 28600.00 & . & $-10.6814^{*}$ & . & . \\
\hline $\mathrm{MCO}-5$ & $08 / 20 / 98$ & $08 / 20 / 98$ & . & 20000 & 18800.00 & . & $6.1856^{*}$ & . & . \\
\hline MCO-6 & $08 / 21 / 98$ & $08 / 21 / 98$ & . & 20000 & 21000.00 & . & $-4.8780^{*}$ & . & . \\
\hline $\mathrm{MCO}-7$ & $08 / 21 / 98$ & $08 / 21 / 98$ & . & 18000 & 18700.00 & . & $-3.8147^{*}$ & . & . \\
\hline TA-50 OUTFALL & $08 / 28 / 98$ & $08 / 28 / 98$ & . & 20000 & 20800.00 & . & $-3.9216^{*}$ & . & . \\
\hline TW-1 & . & $09 / 01 / 98$ & $05 / 28 / 98$ & . & 36300.00 & 34000.00 & . & . & $-6.5434^{*}$ \\
\hline$T W-2 A$ & . & $09 / 01 / 98$ & $09 / 01 / 98$ & . & 69900.00 & 60000.00 & . & . & $-15.2425^{*}$ \\
\hline TW-3 & . & $09 / 01 / 98$ & $09 / 01 / 98$ & . & 3150.00 & 3900.00 & . & . & $21.2766^{*}$ \\
\hline$T W-4$ & & $09 / 01 / 98$ & $09 / 01 / 98$ & & 1980.00 & 3100.00 & . & . & $44.0945^{*}$ \\
\hline TW-8 & $09 / 02 / 98$ & $09 / 02 / 98$ & $09 / 02 / 98$ & 2200 & 2070.00 & 3600.00 & $6.0890^{*}$ & $-48.2759^{*}$ & $53.9683 *$ \\
\hline
\end{tabular}

SUITE $=$ Inorganics Analyte $=F$ Unit $=\mu \mathrm{G} / \mathrm{L}$

\begin{tabular}{|c|c|c|c|c|c|c|c|c|c|}
\hline Location & NM Date & EPA Date & $\begin{array}{l}\text { LANL } \\
\text { Date }\end{array}$ & $\begin{array}{l}\text { NMED } \\
\text { Result }\end{array}$ & $\begin{array}{l}\text { EPA } \\
\text { Result }\end{array}$ & $\begin{array}{l}\text { LANL } \\
\text { Result }\end{array}$ & $\begin{array}{l}\text { NMED vs } \\
\text { EPA }\end{array}$ & $\begin{array}{l}\text { NMED vs } \\
\text { LANL }\end{array}$ & $\begin{array}{l}\text { LANL vs } \\
\text { EPA }\end{array}$ \\
\hline BAYO-1 (OUTFALL) & $09 / 01 / 98$ & $09 / 01 / 98$ & 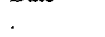 & 1080 & 350.00 & . & $102.098^{*}$ & . & . \\
\hline DP SPRING & 09/02/98 & $09 / 02 / 98$ & . & 1530 & 1060.00 & . & $36.293^{*}$ & . & . \\
\hline $\mathrm{MCO}-5$ & $08 / 20 / 98$ & $08 / 20 / 98$ & . & 1400 & 1270.00 & . & $9.738^{*}$ & . & . \\
\hline $\mathrm{MCO}-6$ & $08 / 21 / 98$ & $08 / 21 / 98$ & . & 1600 & 1460.00 & . & $9.150^{*}$ & . & . \\
\hline $\mathrm{MCO}-7$ & $08 / 21 / 98$ & $08 / 21 / 98$ & . & 1800 & 1560.00 & . & $14.286^{*}$ & . & . \\
\hline TA-50 OUTFALL & $08 / 28 / 98$ & $08 / 28 / 98$ & . & 2500 & 3090.00 & . & $-21.109^{*}$ & . & . \\
\hline TW-1 & & $09 / 01 / 98$ & $05 / 28 / 98$ & . & 350.00 & 420.000 & . & . & $18.1818^{*}$ \\
\hline TW-2A & . & $09 / 01 / 98$ & $09 / 01 / 98$ & . & 240.00 & 200.000 & . & . & $-18.1818^{*}$ \\
\hline TW-3 & . & $09 / 01 / 98$ & $09 / 01 / 98$ & . & 350.00 & 360.000 & . & . & $2.8169^{*}$ \\
\hline TW-4 & & $09 / 01 / 98$ & $09 / 01 / 98$ & . & 180.00 & 180.000 & . & . & $0.0000^{*}$ \\
\hline TW-8 & $09 / 02 / 98$ & $09 / 02 / 98$ & $09 / 02 / 98$ & 130 & 160.00 & 150.000 & $-20.690^{*}$ & $-14.2857^{*}$ & $-6.4516^{*}$ \\
\hline
\end{tabular}

SUTE $=$ Inorganics Analyte $=$ NITRATE $/$ NITRITE Unit $=\mu \mathrm{G} / \mathrm{L}$

\begin{tabular}{|c|c|c|c|c|c|c|c|c|c|}
\hline Location & NM Date & EPA Date & $\begin{array}{l}\text { LANL } \\
\text { Date }\end{array}$ & $\begin{array}{l}\text { NMED } \\
\text { Result }\end{array}$ & $\begin{array}{l}\text { EPA } \\
\text { Result }\end{array}$ & $\begin{array}{l}\text { LANL } \\
\text { Result }\end{array}$ & $\begin{array}{l}\text { NMED vs } \\
\text { EPA }\end{array}$ & $\begin{array}{l}\text { NMED vs } \\
\text { LANL }\end{array}$ & $\begin{array}{l}\text { LANL vs } \\
\text { EPA }\end{array}$ \\
\hline BAYO-1 (OUTFALL) & $09 / 01 / 98$ & $09 / 01 / 98$ & . & 2600 & 3010 & . & $-14.6168^{*}$ & . & . \\
\hline DP SPRING & $09 / 02 / 98$ & $09 / 02 / 98$ & . & 360 & 275 & . & $26.7717 *$ & . & . \\
\hline MCO-5 & $08 / 20 / 98$ & $08 / 20 / 98$ & . & 13000 & 15400 & . & $-16.9014^{*}$ & . & . \\
\hline MCO-6 & $08 / 21 / 98$ & $08 / 21 / 98$ & . & 14000 & 17500 & . & $-22.2222 *$ & . & . \\
\hline $\mathrm{MCO}-7$ & $08 / 21 / 98$ & $08 / 21 / 98$ & - & 15000 & 18200 & - & $-19.2771^{*}$ & . & . \\
\hline TW-1 & $09 / 01 / 98$ & $09 / 01 / 98$ & $05 / 28 / 98$ & 4900 & 5570 & 5270.00 & $-12.7985^{*}$ & $-7.27630^{*}$ & $-5.5351^{*}$ \\
\hline TW-2A & - & $09 / 01 / 98$ & $09 / 01 / 98$ & . & 767 & 400.00 & . & . & $-62.8963 *$ \\
\hline TW-3 & . & $09 / 01 / 98$ & $09 / 01 / 98$ & . & 569 & 650.00 & . & . & $13.2896^{*}$ \\
\hline TW-4 & & $09 / 01 / 98$ & $09 / 01 / 98$ & & 285 & 320.00 & . & . & $11.5702^{*}$ \\
\hline TW-8 & $09 / 02 / 98$ & $09 / 02 / 98$ & $09 / 02 / 98$ & 280 & 226 & 280.00 & $21.3439^{*}$ & $-0.00000^{*}$ & $21.3439 *$ \\
\hline
\end{tabular}

- SUITE $=$ Inorganics Analyte $=$ SO4 Unit $=\mu \mathrm{G} / \mathrm{L}$

\begin{tabular}{|c|c|c|c|c|c|c|c|c|c|}
\hline Location & NM Date & EPA Date & $\begin{array}{l}\text { LANL } \\
\text { Date }\end{array}$ & $\begin{array}{l}\text { NMED } \\
\text { Result }\end{array}$ & $\begin{array}{l}\text { EPA } \\
\text { Result }\end{array}$ & $\begin{array}{l}\text { LANL } \\
\text { Result }\end{array}$ & $\begin{array}{l}\text { NMED vs } \\
\text { EPA }\end{array}$ & $\begin{array}{l}\text { NMED vs } \\
\text { LANL. }\end{array}$ & $\begin{array}{l}\text { LANL vs } \\
\text { EPA }\end{array}$ \\
\hline BAYO-1 (OUTFALL) & $09 / 01 / 98$ & $09 / 01 / 98$ & 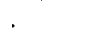 & 24100 & 24600.00 & . & $-2.0534 *$ & 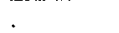 & . \\
\hline DP SPRING & $09 / 02 / 98$ & $09 / 02 / 98$ & . & 6850 & 5770.00 & . & $17.1157^{*}$ & . & . \\
\hline MCO-6 & $08 / 21 / 98$ & $08 / 21 / 98$ & . & 17000 & 17700.00 & . & $-4.0346^{*}$ & . & . \\
\hline $\mathrm{MCO}-7$ & $08 / 21 / 98$ & $08 / 21 / 98$ & . & 16000 & 16300.00 & . & $-1.8576^{*}$ & . & . \\
\hline TA-50 OUTFALL & $08 / 28 / 98$ & $08 / 28 / 98$ & & 98000 & 125000.00 & & $-24.2152 *$ & . & 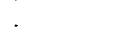 \\
\hline TW-1 & . & $09 / 01 / 98$ & $05 / 28 / 98$ & . & 23200.00 & 22000 & . & . & $-5.3097^{*}$ \\
\hline TW-3 & . & $09 / 01 / 98$ & $09 / 01 / 98$ & . & 2920.00 & 4000 & . & . & $31.2139 \%$ \\
\hline TW-4 & . & $09 / 01 / 98$ & $09 / 01 / 98$ & . & 2090.00 & 3000 & . & . & $35.7564 *$ \\
\hline TW-8 & $09 / 02 / 98$ & $09 / 02 / 98$ & $09 / 02 / 98$ & 2100 & 2030.00 & 3000 & $3.3898 *$ & $-35.2941 *$ & $38.5686^{*}$ \\
\hline
\end{tabular}


SUITE $=$ Inorganics Analyte $=$ TDS Unit $=\mu \mathrm{G} /$

\begin{tabular}{|c|c|c|c|c|c|c|c|c|c|}
\hline Location & NM Date & EPA Date & $\begin{array}{l}\text { LANL } \\
\text { Date }\end{array}$ & $\begin{array}{l}\text { NMED } \\
\text { Result }\end{array}$ & $\begin{array}{l}\text { EPA } \\
\text { Result }\end{array}$ & $\begin{array}{l}\text { LANL } \\
\text { Result }\end{array}$ & $\begin{array}{l}\text { NMED vs } \\
\text { EPA }\end{array}$ & $\begin{array}{l}\text { NMED vs } \\
\text { LANL }\end{array}$ & $\begin{array}{l}\text { LANL vs } \\
\text { EPA }\end{array}$ \\
\hline $\mathrm{MCO}-2$ & $08 / 19 / 98$ & $08 / 19 / 98$ & . & 400000 & 405000 & . & $-1.2422 *$ & . & . \\
\hline $\mathrm{MCO}-3$ & $08 / 20 / 98$ & $08 / 20 / 98$ & . & 560000 & 538000 & $4.0073^{*}$ & . & . & \\
\hline MCO-5 & $08 / 20 / 98$ & $08 / 20 / 98$ & . & 350000 & 404000 & . & $-14.3236^{*}$ & . & . \\
\hline MCO-6 & $08 / 21 / 98$ & $08 / 21 / 98$ & . & 370000 & 374000 & . & $-1.0753^{*}$ & $\therefore$ & . \\
\hline $\mathrm{MCO}-7$ & $08 / 21 / 98$ & $08 / 21 / 98$ & . & 380000 & 381000 & . & $-0.2628 *$ & . & . \\
\hline TW-1 & . & $09 / 01 / 98$ & $05 / 28 / 98$ & . & 777000 & 304000 & . & . & $-87.512^{*}$ \\
\hline$T W-2 A$ & $:$ & $09 / 01 / 98$ & $09 / 01 / 98$ & . & 220000 & 740000 & . & . & $108.333^{*}$ \\
\hline TW-3 & . & $09 / 01 / 98$ & $09 / 01 / 98$ & . & 162000 & 238000 & . & . & $38.000^{*}$ \\
\hline TW-8 & . & $09 / 02 / 98$ & $09 / 02 / 98$ & . & 127000 & 144000 & 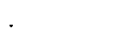 & . & $12.546^{*}$ \\
\hline
\end{tabular}

SUITE $=$ Inorganics Analyte $=$ TOT. KJELDAHL N Unit $=\mu \mathrm{G} / \mathrm{L}$

\begin{tabular}{|c|c|c|c|c|c|c|c|c|c|}
\hline Location & NM Date & EPA Date & $\begin{array}{l}\text { LANL } \\
\text { Date }\end{array}$ & $\begin{array}{l}\text { NMED } \\
\text { Result }\end{array}$ & $\begin{array}{l}\text { EPA } \\
\text { Result }\end{array}$ & $\begin{array}{l}\text { LANL } \\
\text { Result }\end{array}$ & $\begin{array}{l}\text { NMED vs } \\
\text { EPA }\end{array}$ & $\begin{array}{l}\text { NMED vs } \\
\text { LANL }\end{array}$ & $\begin{array}{l}\text { LANL vs } \\
\text { EPA }\end{array}$ \\
\hline BAYO-1 (OUTFALL) & $09 / 01 / 98$ & $09 / 01 / 98$ & . & 15000 & 225000.0 & . & $-175.000^{*}$ & . & \\
\hline DP SPRING & $09 / 02 / 98$ & $09 / 02 / 98$ & . & 200 & 125.0 & . & $46.154^{*}$ & $\because$ & . \\
\hline $\mathrm{MCO}-5$ & $08 / 20 / 98$ & $08 / 20 / 98$ & . & 1400 & $<50.0$ & & 186.207 & . & . \\
\hline $\mathrm{MCO}-7$ & $08 / 21 / 98$ & $08 / 21 / 98$ & . & 1100 & 57.5 & & $180.130^{*}$ & . & . \\
\hline TW-1 & $09 / 01 / 98$ & $09 / 01 / 98$ & . & $<100$ & 113.0 & & -12.207 & . & . \\
\hline TW-8 & $09 / 02 / 98$ & $09 / 02 / 98$ & . & 1700 & $<50.0$ & 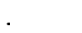 & 188.571 & . & . \\
\hline
\end{tabular}

SUITE $=$ Inorganics Analyte $=$ TSS Unit $=\mu \mathrm{G} / \mathrm{L}$

\begin{tabular}{|c|c|c|c|c|c|c|c|c|c|}
\hline Location & NM Date & EPA Date & $\begin{array}{l}\text { LANL } \\
\text { Date }\end{array}$ & $\begin{array}{l}\text { NMED } \\
\text { Result }\end{array}$ & $\begin{array}{l}\text { EPA } \\
\text { Result }\end{array}$ & $\begin{array}{l}\text { LANL } \\
\text { Result }\end{array}$ & $\begin{array}{l}\text { NMED vs } \\
\text { EPA }\end{array}$ & $\begin{array}{l}\text { NMED vs } \\
\text { LANL }\end{array}$ & $\begin{array}{l}\text { LANL vs } \\
\text { EPA }\end{array}$ \\
\hline APCO-1 & . & $09 / 03 / 98$ & $09 / 03 / 98$ & 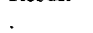 & 4000 & 2000 & 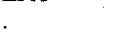 & 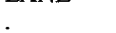 & $-66.667 *$ \\
\hline BASALT SPRING & . & $09 / 09 / 98$ & $06 / 04 / 98$ & . & 1000 & 9000 & . & . & $160.000^{*}$ \\
\hline DP SPRING & . & $09 / 02 / 98$ & $\because \quad 09 / 02 / 98$ & . & 4000 & 1000 & . & . & $-120.000^{*}$ \\
\hline LA MESITA SPRING & . & $09 / 08 / 98$ & $09 / 08 / 98$ & . & 63000 & 49000 & . & . & $-25.000^{*}$ \\
\hline $\mathrm{LAO}-2$ & . & $08 / 31 / 98$ & $08 / 31 / 98$ & . & 9000 & $<1000$ & . & . & -160.000 \\
\hline $\mathrm{LAO}-3 \mathrm{~A}$ & . & $08 / 31 / 98$ & $08 / 31 / 98$ & . & 6000 & $<1000$ & . & . & -142.857 \\
\hline $\mathrm{LAO}-4.5 \mathrm{C}$ & . & $08 / 31 / 98$ & $05 / 14 / 98$ & . & 9000 & 9000 & . & . & $0.000^{*}$ \\
\hline $\mathrm{MCO}-2$ & $08 / 19 / 98$ & $08 / 19 / 98$ & $\because$ & $<20000$ & 91000 & . & -127.928 & . & \\
\hline $\mathrm{MCO}-4 \mathrm{~B}$ & , & $08 / 20 / 98$ & $05 / 27 / 98$ & . & $<1000$ & 8000 & 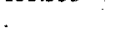 & & 155.556 \\
\hline MCO-5 & $08 / 20 / 98$ & $08 / 20 / 98$ & $05 / 27 / 98$ & $<20000$ & 10000 & 1000 & 66.667 & 180.952 & $-163.636^{*}$ \\
\hline MCO-6 & $08 / 21 / 98$ & $08 / 21 / 98$ & : & $<20000$ & 14000 & . & 35.294 & . & . \\
\hline $\mathrm{MCO}-7$ & $08 / 21 / 98$ & $08 / 21 / 98$ & . & 59000 . & 47000 & . & $22.642^{*}$ & . & . \\
\hline $\mathrm{MT}-3$ & . & $08 / 27 / 98$ & $09 / 04 / 98$ & & 4000 & 1000 & . & . & $-120.000^{*}$ \\
\hline $\mathrm{MT}-4$ & . & $08 / 26 / 98$ & $05 / 14 / 98$ & . & 9000 & 4000 & . & . & $-76.923^{*}$ \\
\hline OTOWI SPRING & . & $09 / 08 / 98$ & $09 / 08 / 98$ & . & 10000 & 5000 & . & . & $-66.667^{*}$ \\
\hline SACRED SPRING & . & $09 / 08 / 98$ & $09 / 08 / 98$ & 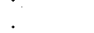 & 11000 & 5000 & & . & $-75.000^{*}$ \\
\hline$T W-1$ & . & $09 / 01 / 98$ & $05 / 28 / 98$ & . & 3000 & 8000 & 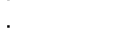 & . & $90.909 *$ \\
\hline TW-3 & . & $09 / 01 / 98$ & $09 / 01 / 98$ & $\dot{.}$ & 2000 & $<1000$ & & . & -66.667 \\
\hline TW-4 & . & $09 / 01 / 98$ & $09 / 01 / 98$ & $r^{\prime}$ & 6000 & 5000 & & . & $-18.182^{*}$ \\
\hline TW-8 & . & $09 / 02 / 98$ & $09 / 02 / 98$ & . & 3000 & $<1000$ & . & 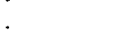 & -100.000 \\
\hline
\end{tabular}

SUITE $=$ Metals Analyte $=\mathrm{AL}$ Unit $=\mu \mathrm{G} / \mathrm{L}$

\begin{tabular}{|c|c|c|c|c|c|c|c|c|c|}
\hline Location & NM Date & EPA Date & $\begin{array}{l}\text { LANL } \\
\text { Date }\end{array}$ & $\begin{array}{l}\text { NMED } \\
\text { Result }\end{array}$ & $\begin{array}{l}\text { EPA } \\
\text { Result }\end{array}$ & $\begin{array}{l}\text { LANL } \\
\text { Result }\end{array}$ & $\begin{array}{l}\text { NMED vs } \\
\text { EPA }\end{array}$ & $\begin{array}{l}\text { NMED vs } \\
\text { LANL }\end{array}$ & $\begin{array}{l}\text { LANL vs } \\
\text { EPA }\end{array}$ \\
\hline APCO-1 & . & $09 / 03 / 98$ & $09 / 03 / 98$ & . & 60.50 & $<50$ & . & . & -19.005 \\
\hline $\mathrm{LAO}-2$ & . & $08 / 31 / 98$ & $08 / 31 / 98$ & . & 746.00 & 423 & . & . & $-55.261^{*}$ \\
\hline LAO-3A & . & $08 / 31 / 98$ & $08 / 31 / 98$ & . & 247.00 & 394 & . & . & $45.866^{*}$ \\
\hline $\mathrm{LAO}-4.5 \mathrm{C}$ & . & $08 / 31 / 98$ & $05 / 14 / 98$ & & 361.00 & 1200 & & . & $107.495^{*}$ \\
\hline MCO-2 & 08/19/98 & $08 / 19 / 98$ & & 8100 & 14900.00 & $=0$ & $-59.130^{*}$ & . & \\
\hline $\mathrm{MCO}-3$ & $08 / 20 / 98$ & $08 / 20 / 98$ & $08 / 20 / 98$ & $<200$ & 65.80 & 359 & 100.978 & -56.8873 & $138.041^{*}$ \\
\hline $\mathrm{MCO}-4 \mathrm{~B}$ & . & $08 / 20 / 98$ & $05 / 27 / 98$ & & 152.00 & 305 & & & $66.958 *$ \\
\hline MCO-5 & $08 / 20 / 98$ & $08 / 20 / 98$ & $05 / 27 / 98$ & $<200$ & 152.00 & 180 & 27.273 & 10.5263 & $16.867 *$ \\
\hline MT-3 & . & $08 / 27 / 98$ & $09 / 04 / 98$ & . & 237.00 & $<50$ & & . & -130.314 \\
\hline MT-4 & . & $08 / 26 / 98$ & $05 / 14 / 98$ & . & 623.00 & 330 & . & . & $-61.490 *$ \\
\hline TW-1 & . & $09 / 01 / 98$ & $05 / 28 / 98$ & . & $<23.60$ & 612 & & . & 185.148 \\
\hline TW-2A & . & $09 / 01 / 98$ & $09 / 01 / 98$ & . & 36.00 & $<50$ & . & . & 32.558 \\
\hline TW-3 & . & $09 / 01 / 98$ & $09 / 01 / 98$ & . & $<23.60$ & $<50$ & . & . & 71.739 \\
\hline TW-4 & . & $09 / 01 / 98$ & $09 / 01 / 98$ & . & $<23.60$ & 85 & . & . & 113.076 \\
\hline TW-8 & . & $09 / 02 / 98$ & $09 / 02 / 98$ & . & $<23.60$ & $<50$ & . & & 71.739 \\
\hline
\end{tabular}


SUITE $=$ Metals Analyte $=$ AS Unit $=\mu G / L$

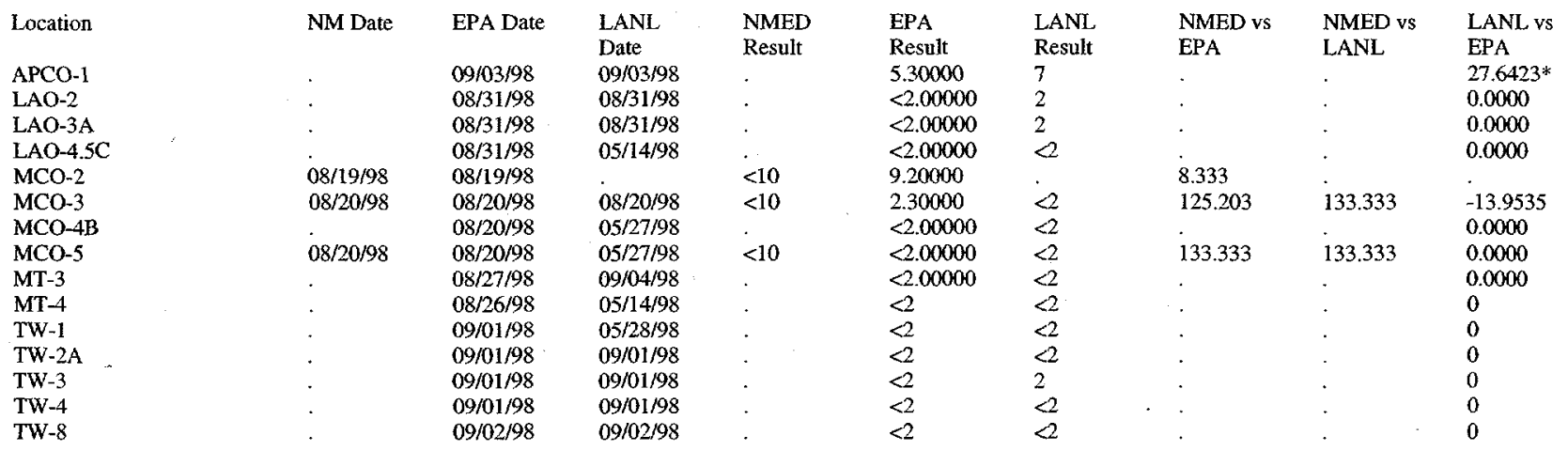

Unfiltered

SUTTE $=$ Metals Analyte $=\mathrm{B}$ Unit $=\mu \mathrm{G} / \mathrm{L}$

\begin{tabular}{|c|c|c|c|c|c|c|c|c|c|}
\hline Lacation & NM Date & EPA Date & $\begin{array}{l}\text { LANL } \\
\text { Date }\end{array}$ & $\begin{array}{l}\text { NMED } \\
\text { Result }\end{array}$ & $\begin{array}{l}\text { EPA } \\
\text { Result }\end{array}$ & $\begin{array}{l}\text { LANL } \\
\text { Result }\end{array}$ & $\begin{array}{l}\text { NMED vs } \\
\text { EPA }\end{array}$ & $\begin{array}{l}\text { NMED vs } \\
\text { LANL }\end{array}$ & $\begin{array}{l}\text { LANL vs } \\
\text { EPA }\end{array}$ \\
\hline APCO-1 & . & $09 / 03 / 98$ & $09 / 03 / 98$ & . & 334.000 & 290 & . & . & $-14.103^{*}$ \\
\hline LAO-2 & . & $08 / 31 / 98$ & $08 / 31 / 98$ & . & 64.500 & 58 & . & . & $-10.612^{*}$ \\
\hline LAO-3A & . & $08 / 31 / 98$ & $08 / 31 / 98$ & . & 62.100 & 47 & . & . & $-27.681 *$ \\
\hline $\mathrm{LAO}-4.5 \mathrm{C}$ & . & $08 / 31 / 98$ & $05 / 14 / 98$ & . & 61.800 & $<20$ & . & . & -102.200 \\
\hline $\mathrm{MCO}-2$ & $08 / 19 / 98$ & $08 / 19 / 98$ & . & $<100$ & 84.000 & . & 17.3913 & . & . \\
\hline $\mathrm{MCO}-3$ & $08 / 20 / 98$ & $08 / 20 / 98$ & $08 / 20 / 98$ & 100 & 179.000 & 173 & $-56.6308^{*}$ & $-53.4799 *$ & $-3.409^{*}$ \\
\hline $\mathrm{MCO}-4 \mathrm{~B}$ & . & $08 / 20 / 98$ & $05 / 27 / 98$ & . & 70.800 & 69 & . & . & $-2.575^{*}$ \\
\hline $\mathrm{MCO}-5$ & $08 / 20 / 98$ & $08 / 20 / 98$ & $05 / 27 / 98$ & $<100$ & 111.000 & 73 & -10.4265 & 31.2139 & $-41.304^{*}$ \\
\hline MT-3 & . & $08 / 27 / 98$ & $09 / 04 / 98$ & . & 109.000 & 82 & . & . & $-28.272^{*}$ \\
\hline MT-4 & . & $08 / 26 / 98$ & $05 / 14 / 98$ & . & 105.000 & 57 & . & . & $-59.259 *$ \\
\hline TW-1 & . & $09 / 01 / 98$ & $05 / 28 / 98$ & . & 112.000 & 62 & . & . & $-57.471 *$ \\
\hline TW- $2 A$ & . & $09 / 01 / 98$ & $09 / 01 / 98$ & . & 114.000 & 85 & . & . & $-29.146^{*}$ \\
\hline TW-3 & . & $09 / 01 / 98$ & $09 / 01 / 98$ & . & 51.000 & 47 & . & . & $-8.163^{*}$ \\
\hline TW-4 & . & $09 / 01 / 98$ & $09 / 01 / 98$ & . & 40.200 & $<20$ & . & . & -67.110 \\
\hline TW-8 & . & $09 / 02 / 98$ & $09 / 02 / 98$ & - & 36.600 & 33 & . & . & $-10.345^{*}$ \\
\hline
\end{tabular}

SUITE $=$ Metals Analyte $=$ BA Unit $=\mu G / L$

\begin{tabular}{|c|c|c|c|c|c|c|c|c|c|}
\hline Location & NM Date & EPA Date & $\begin{array}{l}\text { LANL } \\
\text { Date }\end{array}$ & $\begin{array}{l}\text { NMED } \\
\text { Result }\end{array}$ & $\begin{array}{l}\text { EPA } \\
\text { Result }\end{array}$ & $\begin{array}{l}\text { LANL } \\
\text { Result }\end{array}$ & $\begin{array}{l}\text { NMED vs } \\
\text { EPA }\end{array}$ & $\begin{array}{l}\text { NMED vs } \\
\text { LANL }\end{array}$ & $\begin{array}{l}\text { LANL vs } \\
\text { EPA }\end{array}$ \\
\hline APCO-1 & . & $09 / 03 / 98$ & $09 / 03 / 98$ & . & 44.900 & 57 & . & 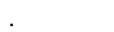 & $23.7488^{*}$ \\
\hline LAO-2 & . & $08 / 31 / 98$ & $08 / 31 / 98$ & . & 54.400 & 56 & . & . & $2.8985^{*}$ \\
\hline LAO-3A & . & $08 / 31 / 98$ & $08 / 31 / 98$ & . & 47.300 & 54 & . & . & $13.2280^{*}$ \\
\hline LAO $-4.5 \mathrm{C}$ & & $08 / 31 / 98$ & $05 / 14 / 98$ & & 40.800 & 39 & & . & $-4.5113^{*}$ \\
\hline $\mathrm{MCO}-2$ & $08 / 19 / 98$ & $08 / 19 / 98$ & . & 200 & 173.000 & $\cdot$ & $14.4772^{*}$ & . & - \\
\hline $\mathrm{MCO}-3$ & $08 / 20 / 98$ & $08 / 20 / 98$ & $08 / 20 / 98$ & $<100$ & 62.700 & 65 & 45.8513 & 42.4242 & $3.6022^{*}$ \\
\hline $\mathrm{MCO}-4 \mathrm{~B}$ & 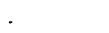 & $08 / 20 / 98$ & $05 / 27 / 98$ & . & 77.000 & 88 & & . & $13.3333^{*}$ \\
\hline $\mathrm{MCO}-5$ & $08 / 20 / 98$ & $08 / 20 / 98$ & $05 / 27 / 98$ & $<100$ & 93.800 & 90 & 6.3983 & 10.5263 & $-4.1349^{*}$ \\
\hline MT -4 & . & $08 / 26 / 98$ & $05 / 14 / 98$ & . & 109.000 & 110 & . & . & $0.9132 *$ \\
\hline TW-I & . & $09 / 01 / 98$ & $05 / 28 / 98$ & . & 74.400 & 87 & . & . & $15.6134^{*}$ \\
\hline TW-2A & . & $09 / 01 / 98$ & $09 / 01 / 98$ & . & 40.800 & 42 & . & . & $2.8986^{*}$ \\
\hline TW-3 & . & $09 / 01 / 98$ & $09 / 01 / 98$ & . & 23.400 & 26 & . & . & $10.5263^{*}$ \\
\hline$T W-4$ & . & $09 / 01 / 98$ & $09 / 01 / 98$ & . & 58.200 & 63 & . & . & $7.9208 *$ \\
\hline TW-8 & . & $09 / 02 / 98$ & $09 / 02 / 98$ & . & 7.000 & 8 & . & . & $13.3333^{*}$ \\
\hline
\end{tabular}

SUITE $=$ Metals Analyte $=$ BE Unit $=\mu \mathrm{G} / \mathrm{L}$

\begin{tabular}{|c|c|c|c|c|c|c|c|c|c|}
\hline Location & NM Date & EPA Date & $\begin{array}{l}\text { LANL } \\
\text { Date }\end{array}$ & $\begin{array}{l}\text { NMED } \\
\text { Result }\end{array}$ & $\begin{array}{l}\text { EPA } \\
\text { Result }\end{array}$ & $\begin{array}{l}\text { LANL } \\
\text { Result }\end{array}$ & $\begin{array}{l}\text { NMED vs } \\
\text { EPA }\end{array}$ & $\begin{array}{l}\text { NMED ys } \\
\text { LANL }\end{array}$ & $\begin{array}{l}\text { LANL vs } \\
\text { EPA }\end{array}$ \\
\hline APCO-1 & . & $09 / 03 / 98$ & $09 / 03 / 98$ & . & $<0.60000$ & $<3$ & . & . & 133.333 \\
\hline $\mathrm{LAO}-2$ & . & $08 / 31 / 98$ & $08 / 31 / 98$ & . & $<0.60000$ & $<3$ & . & . & 133.333 \\
\hline $\mathrm{LAO}-3 \mathrm{~A}$ & . & $08 / 31 / 98$ & $08 / 31 / 98$ & . & $<0.60000$ & $<3$ & . & $\therefore$ & 133.333 \\
\hline $\mathrm{LAO}-4.5 \mathrm{C}$ & . & $08 / 31 / 98$ & $05 / 14 / 98$ & . & 0.64000 & $<3$ & . & . & 129.670 \\
\hline $\mathrm{MCO}-2$ & $08 / 19 / 98$ & $08 / 19 / 98$ & . & $<5$ & 1.90000 & . & 89.855 & . & . \\
\hline $\mathrm{MCO}-3$ & $08 / 20 / 98$ & $08 / 20 / 98$ & $08 / 20 / 98$ & $<5$ & $<0.60000$ & $<3$ & 157.143 & 50 & 133.333 \\
\hline $\mathrm{MCO}-4 \mathrm{~B}$ & & $08 / 20 / 98$ & $05 / 27 / 98$ & . & $<0.60000$ & $<3$ & . & . & 133.333 \\
\hline MCO-5 & $08 / 20 / 98$ & $08 / 20 / 98$ & $05 / 27 / 98$ & $<5$ & $<0.60000$ & $<3$ & 157.143 & 50 & 133.333 \\
\hline $\mathrm{MT}-4$ & . & $08 / 26 / 98$ & $05 / 14 / 98$ & . & $<0.60000$ & $<3$ & . & . & 133.333 \\
\hline TW-1 & . & $09 / 01 / 98$ & $05 / 28 / 98$ & . & $<0.60000$ & $<3$ & . & . & 133.333 \\
\hline TW-2A & . & 09/01/98 & $09 / 01 / 98$ & . & $<6.00000$ & $<3$ & . & . & -66.667 \\
\hline TW-3 & . & $09 / 01 / 98$ & $09 / 01 / 98$ & . & $<0.60000$ & $<3$ & . & . & 133.333 \\
\hline TW-4 & . & $09 / 01 / 98$ & $09 / 01 / 98$ & . & $<0.60000$ & $<3$ & . & . & 133.333 \\
\hline TW-8 & - & $09 / 02 / 98$ & $09 / 02 / 98$ & . & $<0.60000$ & $<3$ & . & . & 133.333 \\
\hline
\end{tabular}


SUITE $=$ Metals Analyte $=$ CA Unit $=\mu G / L$

\begin{tabular}{|c|c|c|c|c|c|c|c|c|c|}
\hline Location & NM Date & EPA Date & $\begin{array}{l}\text { LANL } \\
\text { Date }\end{array}$ & $\begin{array}{l}\text { NMED } \\
\text { Result }\end{array}$ & $\begin{array}{l}\text { EPA } \\
\text { Result }\end{array}$ & $\begin{array}{l}\text { LANL } \\
\text { Result }\end{array}$ & $\begin{array}{l}\text { NMED vs } \\
\text { EPA }\end{array}$ & $\begin{array}{l}\text { NMED vs } \\
\text { LANL }\end{array}$ & $\begin{array}{l}\text { LANL vs } \\
\text { EPA }\end{array}$ \\
\hline TW-8 & $09 / 02 / 98$ & .. & $09 / 02 / 98$ & 11000 & . & 11605.00 & . & $-5.35279 *$ & . \\
\hline
\end{tabular}

- SUTTE $=$ Metals Analyte $=\mathrm{CR}$ Unit $=\mu \mathrm{G} / \mathrm{L}$

\begin{tabular}{|c|c|c|c|c|c|c|c|c|c|}
\hline Location & NM Date & EPA Date & $\begin{array}{l}\text { LANL } \\
\text { Date }\end{array}$ & $\begin{array}{l}\text { NMED } \\
\text { Result }\end{array}$ & $\begin{array}{l}\text { EPA } \\
\text { Result }\end{array}$ & $\begin{array}{l}\text { LANL } \\
\text { Result }\end{array}$ & $\begin{array}{l}\text { NMED vs } \\
\text { EPA }\end{array}$ & $\begin{array}{l}\text { NMED vs } \\
\text { LANL }\end{array}$ & $\begin{array}{l}\text { LANL vs } \\
\text { EPA }\end{array}$ \\
\hline APCO-1 & . & $09 / 03 / 98$ & $09 / 03 / 98$ & . & $<4,2000$ & $<7$ & . & . & 50.000 \\
\hline LAO-2 & . & $08 / 31 / 98$ & $08 / 31 / 98$ & . & $<4.2000$ & $<7$ & . & . & 50.000 \\
\hline LAO-3A & . & $08 / 31 / 98$ & $08 / 31 / 98$ & . & $<4.2000$ & $<7$ & . & . & 50.000 \\
\hline LAO-4.5C & . & $08 / 31 / 98$ & $05 / 14 / 98$ & . & $<4.2000$ & 12 & . & & 96.296 \\
\hline $\mathrm{MCO}-3$ & . & $08 / 20 / 98$ & $08 / 20 / 98$ & . & $<4.2000$ & $<7$ & . & . & 50.000 \\
\hline $\mathrm{MCO}-4 \mathrm{~B}$ & . & $08 / 20 / 98$ & $05 / 27 / 98$ & . & $<4.2000$ & $<7$ & . & . & 50.000 \\
\hline $\mathrm{MCO}-5$ & . & $08 / 20 / 98$ & $05 / 27 / 98$ & . & $<4.2000$ & 21 & . & . & 133.333 \\
\hline $\mathrm{MT}-4$ & . & $08 / 26 / 98$ & $05 / 14 / 98$ & . & $<4.2000$ & 9 & . & . & 72.727 \\
\hline TW-1 & . & $09 / 01 / 98$ & $05 / 28 / 98$ & . & $<4.2000$ & $<7$ & . & . & 50.000 \\
\hline TW-2A & . & $09 / 01 / 98$ & $09 / 01 / 98$ & . & $<4.2000$ & $<7$ & . & . & 50.000 \\
\hline TW-3 & & 09/01/98 & $09 / 01 / 98$ & . & 16.8000 & $<7$ & . & . & -82.353 \\
\hline$T W-4$ & . & $09 / 01 / 98$ & $09 / 01 / 98$ & . & 49.0000 & $<7$ & . & . & -150.000 \\
\hline TW-8 & & $09 / 02 / 98$ & $09 / 02 / 98$ & & $<4.2000$ & $<7$ & . & & 50.000 \\
\hline
\end{tabular}

Unfiltered

- SUITE $=$ Metals Analyte $=$ CU Unit $=\mu G / L$

\begin{tabular}{|c|c|c|c|c|c|c|c|c|c|}
\hline Location & NM Date & EPA Date & $\begin{array}{l}\text { LANL } \\
\text { Date }\end{array}$ & $\begin{array}{l}\text { NMED } \\
\text { Result }\end{array}$ & $\begin{array}{l}\text { EPA } \\
\text { Result }\end{array}$ & $\begin{array}{l}\text { LANL } \\
\text { Result }\end{array}$ & $\begin{array}{l}\text { NMED vs } \\
\text { EPA }\end{array}$ & $\begin{array}{l}\text { NMED vs } \\
\text { LANL }\end{array}$ & $\begin{array}{l}\text { LANL vs } \\
\text { EPA }\end{array}$ \\
\hline APCO-1 & . & $09 / 03 / 98$ & $09 / 03 / 98$ & . & 7.4000 & $<10$ & 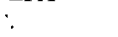 & . & 29.8851 \\
\hline LAO-2 & . & $08 / 31 / 98$ & $08 / 31 / 98$ & . & $<6.2000$ & $<10$ & . & . & 46.9136 \\
\hline LAO-3A & . & $08 / 31 / 98$ & $08 / 31 / 98$ & . & $<6.2000$ & $<10$ & . & . & 46.9136 \\
\hline $\mathrm{LAO}-4.5 \mathrm{C}$ & . & $08 / 31 / 98$ & $05 / 14 / 98$ & . & 8.2000 & $<10$ & . & . & 19.7802 \\
\hline $\mathrm{MCO}-3$ & . & $08 / 20 / 98$ & $08 / 20 / 98$ & . & 14.9000 & 18 & . &. & $18.8450^{*}$ \\
\hline $\mathrm{MCO}-4 \mathrm{~B}$ & . & $08 / 20 / 98$ & $05 / 27 / 98$ & . & $<6.2000$ & $<10$ & . & . & 46.9136 \\
\hline MCO-5 & . & $08 / 20 / 98$ & $05 / 27 / 98$ & . & 7.0000 & $<10$ & . & . & 35.2941 \\
\hline MT-3 & i. & $08 / 27 / 98$ & $09 / 04 / 98$ & i. & 9.3000 & $<10$ & . & . & 7.2539 \\
\hline MT-4 & . & $08 / 26 / 98$ & $05 / 14 / 98$ & . & 16.6000 & $<10$ & . & $\therefore$ & -49.6241 \\
\hline TW-1 & . & $09 / 01 / 98$ & $05 / 28 / 98$ & . & $<6.2000$ & $<10$ & . & . & 46.9136 \\
\hline TW-2A & . & $09 / 01 / 98$ & $09 / 01 / 98$ & . & $<6.2000$ & $<10$ & . & . & 46.9136 \\
\hline TW-3 & . & $09 / 01 / 98$ & $09 / 01 / 98$ & . & $<6.2000$ & $<10$ & . & . & 46.9136 \\
\hline TW-4 & . & $09 / 01 / 98$ & $09 / 01 / 98$ & . & 13.0000 & 31 & . & . & $81.8182^{*}$ \\
\hline TW-8 & . & $09 / 02 / 98$ & $09 / 02 / 98$ & . & $<6.2000$ & $<10$ & 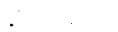 & . & 46.9136 \\
\hline
\end{tabular}

SUTTE $=$ Metals Analyte $=$ FE Unit $=\mu \mathrm{G} / \mathrm{L}$

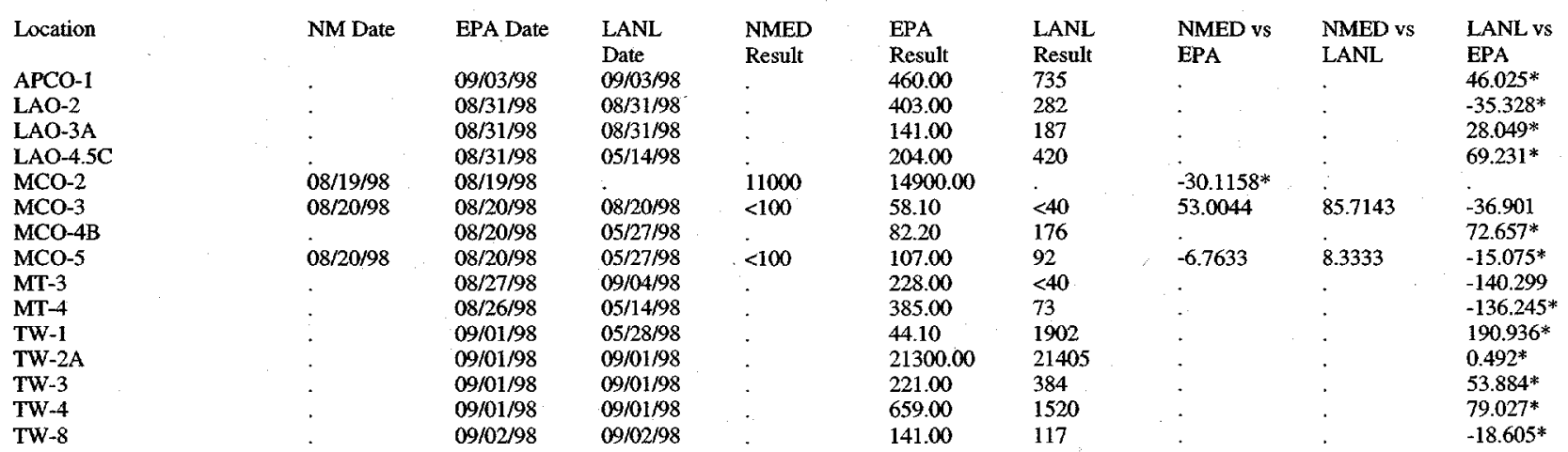

SUITE $=$ Metals Analyte $=$ HG Unit $=\mu \mathrm{G} / \mathrm{L}$

\begin{tabular}{|c|c|c|c|c|c|c|c|c|c|}
\hline Location & NM Date & EPA Date & $\begin{array}{l}\text { LANL } \\
\text { Date }\end{array}$ & $\begin{array}{l}\text { NMED } \\
\text { Result }\end{array}$ & $\begin{array}{l}\text { EPA } \\
\text { Result }\end{array}$ & $\begin{array}{l}\text { LANL } \\
\text { Result }\end{array}$ & $\begin{array}{l}\text { NMED vs } \\
\text { EPA }\end{array}$ & $\begin{array}{l}\text { NMED vs } \\
\text { LANL }\end{array}$ & $\begin{array}{l}\text { LANL vs } \\
\text { EPA }\end{array}$ \\
\hline$A P C O-I$ & - & $09 / 03 / 98$ & $09 / 03 / 98$ & . & $<0.10000$ & $<0.20000$ & . $\quad$. & . & 66.6667 \\
\hline BASALT SPRING & . & $09 / 09 / 98$ & $06 / 04 / 98$ & . & $<0.10000$ & $<0.20000$ & . & . & 66.6667 \\
\hline DP SPRING & . & $09 / 02 / 98$ & $09 / 02 / 98$ & . & $<0.10000$ & $<0.20000$ & . & . & 66.6667 \\
\hline$\angle A O-2$ & - & $08 / 31 / 98$ & $08 / 31 / 98$ & . & $<0.10000$ & $<0.20000$ & - & - & 66.6667 \\
\hline$L A O-3 A$ & . & $08 / 31 / 98$ & $08 / 31 / 98$ & . & $<0.10000$ & $<0.20000$ & - & - & 66.6667 \\
\hline$L A O-4.5 C$ & - & $08 / 31 / 98$ & $05 / 14 / 98$ & - & $<0.10000$ & $<0.20000$ & - & . & 66.6667 \\
\hline$M C O-3$ & . & $08 / 20 / 98$ & $08 / 20 / 98$ & . & $<0.10000$ & $<0.20000$ & - & - & 66.6667 \\
\hline$M C O-4 B$ & - & $08 / 20 / 98$ & $05 / 27 / 98$ & . & $<0.10000$ & $<0.20000$ & - & - & 66.6667 \\
\hline$M C O-5$ & - & $08 / 20 / 98$ & $05 / 27 / 98$ & . & $<0.10000$ & $<0.20000$ & - & - & 66.6667 \\
\hline$M T-3$ & . & $08 / 27 / 98$ & $09 / 04 / 98$ & . & $<0.10000$ & $<0.20000$ & . & - & 66.6667 \\
\hline$M T-4$ & . & $08 / 26 / 98$ & $05 / 14 / 98$ & . & $<0.10000$ & $<0.20000$ & . & . & 66.6667 \\
\hline OTOWI SPRING & . & $09 / 08 / 98$ & 09/08/98 & . & $<0.10000$ & $<0.20000$ & , & . & 66.6667 \\
\hline TW-1 & . & $09 / 01 / 98$ & $05 / 28 / 98$ & . & $<0.10000$ & $<0.20000$ & . & . & 66.6667 \\
\hline TW-2A & . & $09 / 01 / 98$ & $09 / 01 / 98$ & . & $<0.10000$ & $<0.20000$ & . & . & 66.6667 \\
\hline TW-3 & . & $09 / 01 / 98$ & 09/01/98 & . & $<0.10000$ & $<0.20000$ & . & . & 66.6667 \\
\hline TW-4 & ${ }^{\circ}$ & $09 / 01 / 98$ & $09 / 01 / 98$ & . & $<0.10000$ & $<0.20000$ & . & . & 66.6667 \\
\hline TW-8 & . & $09 / 02 / 98$ & $09 / 02 / 98$ & ${ }^{*}$ & $<0.10000$ & $<0.20000$ & . & . & 66.6667 \\
\hline
\end{tabular}


SUITE $=$ Metals Analyte $=\mathrm{K}$ Unit $=\mu \mathrm{G} / \mathrm{L}$

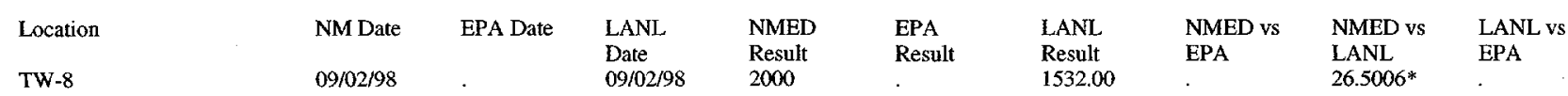

SUITE $=$ Metals Analyte $=$ MG Unit $=\mu G / L$

\begin{tabular}{|c|c|c|c|c|c|c|c|c|c|}
\hline Location & NM Date & EPA Date & $\begin{array}{l}\text { LANL } \\
\text { Date }\end{array}$ & $\begin{array}{l}\text { NMED } \\
\text { Result }\end{array}$ & $\begin{array}{l}\text { EPA } \\
\text { Result }\end{array}$ & $\begin{array}{l}\text { LANL } \\
\text { Result }\end{array}$ & $\begin{array}{l}\text { NMED vs } \\
\text { EPA }\end{array}$ & $\begin{array}{l}\text { NMED vs } \\
\text { LANL }\end{array}$ & $\begin{array}{l}\text { LANL vs } \\
\text { EPA }\end{array}$ \\
\hline TW-8 & $09 / 02 / 98$ & . & $09 / 02 / 98$ & 4000 & & 3939.00 & . & $1.53672 *$ & . \\
\hline
\end{tabular}

SUTTE $=$ Metals Analyte $=$ MN Unit $=\mu \mathrm{G} / \mathrm{L}$

\begin{tabular}{|c|c|c|c|c|c|c|c|c|c|}
\hline Location & NM Date & EPA Date & $\begin{array}{l}\text { LANL } \\
\text { Date }\end{array}$ & $\begin{array}{l}\text { NMED } \\
\text { Result }\end{array}$ & $\begin{array}{l}\text { EPA } \\
\text { Result }\end{array}$ & $\begin{array}{l}\text { LANL } \\
\text { Result }\end{array}$ & $\begin{array}{l}\text { NMED vs } \\
\text { EPA }\end{array}$ & $\begin{array}{l}\text { NMED vs } \\
\text { LANL }\end{array}$ & $\begin{array}{l}\text { LANL vs } \\
\text { EPA }\end{array}$ \\
\hline APCO-1 & . & $09 / 03 / 98$ & $09 / 03 / 98$ & . & 925.00 & 1008 & . & . & $8.5877^{*}$ \\
\hline $\mathrm{LAO}-2$ & . & $08 / 31 / 98$ & $08 / 31 / 98$ & . & 3.80 & 2 & . & . & $-62.0690^{*}$ \\
\hline LAO-3A & . & $08 / 31 / 98$ & $08 / 31 / 98$ & . & 1.30 & $<2$ & . & . & 42.4242 \\
\hline LAO-4.5C & & $08 / 31 / 98$ & $05 / 14 / 98$ & . & 3.40 & 3 & . & . & $-12.5000^{*}$ \\
\hline $\mathrm{MCO}_{2} 2$ & $08 / 19 / 98$ & $08 / 19 / 98$ & & 1300 & 13200.00 & . & $-164.138^{*}$ & 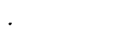 & . \\
\hline $\mathrm{MCO}-3$ & $08 / 20 / 98$ & $08 / 20 / 98$ & $08 / 20 / 98$ & $<10$ & 5.40 & $<2$ & 59.740 & 133.333 & -91.8919 \\
\hline $\mathrm{MCO}-4 \mathrm{~B}$ & . & $08 / 20 / 98$ & $05 / 27 / 98$ & . & 2.40 & 2 & . & 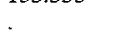 & $-18.1818^{*}$ \\
\hline MCO-5 & $08 / 20 / 98$ & $08 / 20 / 98$ & $05 / 27 / 98$ & $<10$ & 2.50 & $<2$ & 120.000 & 133.333 & -22.2222 \\
\hline MT-3 & . & $08 / 27 / 98$ & $09 / 04 / 98$ & 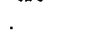 & 3.50 & $<2$ & . & . & -54.5455 \\
\hline MT-4 & . & $08 / 26 / 98$ & $05 / 14 / 98$ & . & 6.50 & 3 & . & . & $-73.6842^{*}$ \\
\hline TW-1 & . & $09 / 01 / 98$ & $05 / 28 / 98$ & . & 41.30 & 65 & . & . & $44.5908^{*}$ \\
\hline TW-2A & . & $09 / 01 / 98$ & $09 / 01 / 98$ & . & 377.00 & 370 & . & . & $-1.8742^{*}$ \\
\hline TW-3 & . & $09 / 01 / 98$ & $09 / 01 / 98$ & . & 8.70 & 13 & . & . & $39.6313^{*}$ \\
\hline$T_{-4}$ & . & $09 / 01 / 98$ & $09 / 01 / 98$ & . & 37.70 & 45 & . & . & $17.6542^{*}$ \\
\hline TW-8 & i. & $09 / 02 / 98$ & $09 / 02 / 98$ & . & 3.60 & 4 & . & . & $10.5263^{*}$ \\
\hline
\end{tabular}

SUITE $=$ Metals Analyte $=$ MO Unit $=\mu G / L$

\begin{tabular}{|c|c|c|c|c|c|c|c|c|c|}
\hline Location & NM Date & EPA Date & $\begin{array}{l}\text { LANL } \\
\text { Date }\end{array}$ & $\begin{array}{l}\text { NMED } \\
\text { Result }\end{array}$ & $\begin{array}{l}\text { EPA } \\
\text { Result }\end{array}$ & $\begin{array}{l}\text { LANL } \\
\text { Result }\end{array}$ & $\begin{array}{l}\text { NMED vs } \\
\text { EPA }\end{array}$ & $\begin{array}{l}\text { NMED vs } \\
\text { LANL }\end{array}$ & $\begin{array}{l}\text { LANL vs } \\
\text { EPA }\end{array}$ \\
\hline APCO-1 & . & $09 / 03 / 98$ & $09 / 03 / 98$ & . & 8.000 & $<30.000$ & . & . & 115.789 \\
\hline LAO-2 & . & $08 / 31 / 98$ & $08 / 31 / 98$ & . & 151.000 & 166.000 & . & . & $9.464^{*}$ \\
\hline LAO-3A & . & $08 / 31 / 98$ & $08 / 31 / 98$ & . & 315.000 & 335.000 & . & . & $6.154^{*}$ \\
\hline LAO-4.5C & . & $08 / 31 / 98$ & $05 / 14 / 98$ & . & 11.000 & $<30.000$ & . & . & 92.683 \\
\hline $\mathrm{MCO}-3$ & . & $08 / 20 / 98$ & $08 / 20 / 98$ & . & 153.000 & 158.000 & . & . & $3.215^{*}$ \\
\hline $\mathrm{MCO}-4 \mathrm{~B}$ & . & $08 / 20 / 98$ & $05 / 27 / 98$ & . & 124.000 & 121.000 & . & . & $-2.449^{*}$ \\
\hline $\mathrm{MCO}-5$ & . & $08 / 20 / 98$ & $05 / 27 / 98$ & . & 113.000 & 127.000 & . & . & $11.667 *$ \\
\hline $\mathrm{MT}-4$ & . & $08 / 26 / 98$ & $05 / 14 / 98$ & . & 33.000 & $<30.000$ & . & . & -9.524 \\
\hline TW-1 & . & $09 / 01 / 98$ & $05 / 28 / 98$ & . & $<7.300$ & $<30.000$ & . & . & 121.716 \\
\hline TW-2A & . & $09 / 01 / 98$ & $09 / 01 / 98$ & . & $<7.300$ & $<30.000$ & . & . & 121.716 \\
\hline TW-3 & . & $09 / 01 / 98$ & $09 / 01 / 98$ & . & $<7.300$ & $<30.000$ & . & . & 121.716 \\
\hline TW-4 & . & $09 / 01 / 98$ & $09 / 01 / 98$ & . & $<7.300$ & $<30.000$ & . & . & 121.716 \\
\hline TW-8 & . & $09 / 02 / 98$ & $09 / 02 / 98$ & . & $<7.300$ & $<30.000$ & . & . & 121.716 \\
\hline
\end{tabular}

SUITE $=$ Metals Analyte $=$ NA Unit $=\mu \mathrm{G} / \mathrm{L}$

\begin{tabular}{|c|c|c|c|c|c|c|c|c|c|}
\hline Location & NM Date & EPA Date & $\begin{array}{l}\text { LANL } \\
\text { Date }\end{array}$ & $\begin{array}{l}\text { NMED } \\
\text { Result }\end{array}$ & $\begin{array}{l}\text { EPA } \\
\text { Result }\end{array}$ & $\begin{array}{l}\text { LANL } \\
\text { Result }\end{array}$ & $\begin{array}{l}\text { NMED vs } \\
\text { EPA }\end{array}$ & $\begin{array}{l}\text { NMED vs } \\
\text { LANL }\end{array}$ & $\begin{array}{l}\text { LANL vs } \\
\text { EPA }\end{array}$ \\
\hline TW-8 & $09 / 02 / 98$ & . & $09 / 02 / 98$ & 9000 & . & 10569.00 & . & $-16.0356^{*}$ & \\
\hline
\end{tabular}

SUITE $=$ Metals Analyte $=P B$ Unit $=\mu G / L$

\begin{tabular}{|c|c|c|c|c|c|c|c|c|c|}
\hline Location & NM Date & EPA Date & $\begin{array}{l}\text { LANL } \\
\text { Date }\end{array}$ & $\begin{array}{l}\text { NMED } \\
\text { Result }\end{array}$ & $\begin{array}{l}\text { EPA } \\
\text { Result }\end{array}$ & $\begin{array}{l}\text { LANL } \\
\text { Result }\end{array}$ & $\begin{array}{l}\text { NMED vs } \\
\text { EPA }\end{array}$ & $\begin{array}{l}\text { NMED vs } \\
\text { LANL }\end{array}$ & $\begin{array}{l}\text { LANL vs } \\
\text { EPA }\end{array}$ \\
\hline APCO-1 & . & $09 / 03 / 98$ & $09 / 03 / 98$ & . & $<1.60000$ & $<3$ & . & . & 60.8696 \\
\hline LA MESITA SPRING & - & $09 / 08 / 98$ & $09 / 08 / 98$ & . & 2.00000 & $<3$ & . & . & 40.0000 \\
\hline LAO-2 & . & $08 / 31 / 98$ & $08 / 31 / 98$ & . & $<1.6000$ & $<3$ & . & . & 60.870 \\
\hline LAO-3A & . & $08 / 31 / 98$ & $08 / 31 / 98$ & . & $<1.6000$ & 16 & . & . & 163.636 \\
\hline $\mathrm{LAO}-4.5 \mathrm{C}$ & . & $08 / 31 / 98$ & $05 / 14 / 98$ & 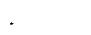 & $<1.6000$ & $<3$ & . & . & 60.870 \\
\hline $\mathrm{MCO}-2$ & $08 / 19 / 98$ & $08 / 19 / 98$ & . & 3 & 5.6000 & . & $-60.4651 *$ & & \\
\hline $\mathrm{MCO}-3$ & $08 / 20 / 98$ & $08 / 20 / 98$ & $08 / 20 / 98$ & $<3$ & $<1.6000$ & $<3$ & 60.8696 & 0 & 60.870 \\
\hline $\mathrm{MCO}-4 \mathrm{~B}$ & . & $08 / 20 / 98$ & $05 / 27 / 98$ & . & $<1.6000$ & $<3$ & . & & 60.870 \\
\hline MCO-5 & $08 / 20 / 98$ & $08 / 20 / 98$ & $05 / 27 / 98$ & $<3$ & $<1.6000$ & $<3$ & 60.8696 & 0 & 60.870 \\
\hline MT-3 & . & $08 / 27 / 98$ & $09 / 04 / 98$ & . & $<1.6000$ & $<3$ & . & . & 60.870 \\
\hline MT-4 & . & $08 / 26 / 98$ & $05 / 14 / 98$ & : & 3.5000 & $<3$ & . & . & -15.385 \\
\hline SACRED SPRING & . & $09 \% 08 / 98$ & $09 / 08 / 98$ & - & $<0.9000$ & $<3$ & . & . & 107.692 \\
\hline TW-1 & . & $09 / 01 / 98$ & $05 / 28 / 98$ & . & 4.0000 & 97 & . & . & $184.158^{*}$ \\
\hline TW-2A & . & $09 / 01 / 98$ & $09 / 01 / 98$ & . & 28.4000 & 53 & . & . & $60.442^{*}$ \\
\hline TW-3 & . & $09 / 01 / 98$ & $09 / 01 / 98$ & . & $<1.6000$ & 3 & . & . & 60.870 \\
\hline TW-4 & . & $09 / 01 / 98$ & $09 / 01 / 98$ & . & 19.4000 & 46 & . & . & $81.346^{*}$ \\
\hline TW-8 & . & $09 / 02 / 98$ & $09 / 02 / 98$ & . & 4.1000 & 5 & . & & $19.780^{*}$ \\
\hline
\end{tabular}


SUITE $=$ Metals Analyte $=S E$ Unit $=\mu G / L$

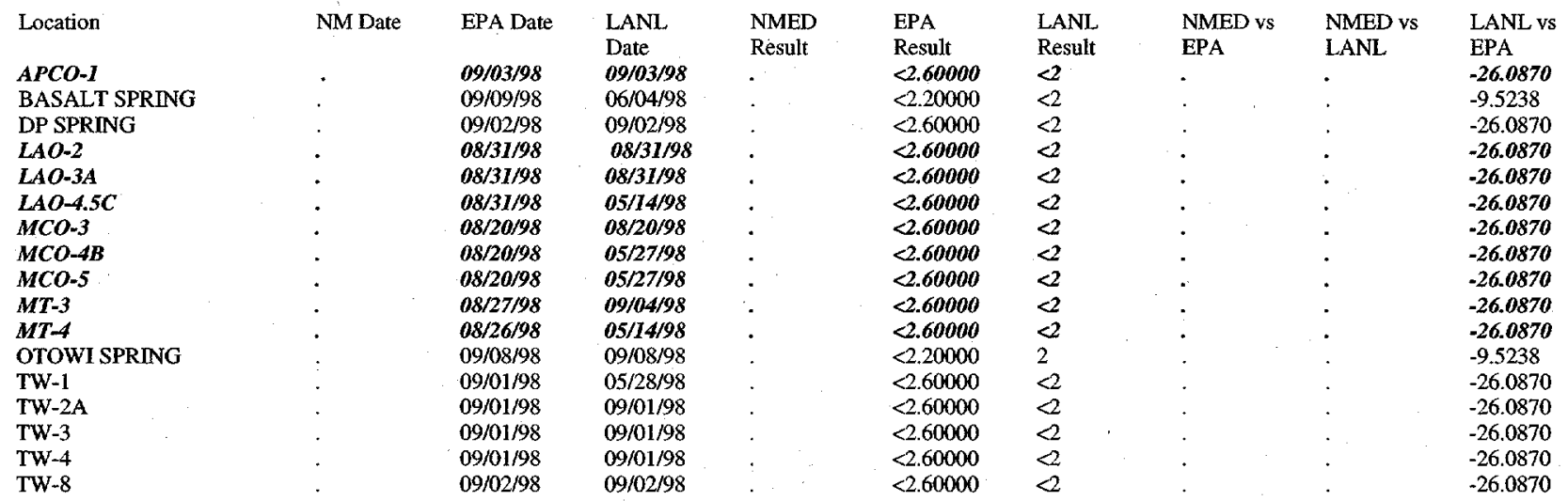

SUITE $=$ Metals Analyte $=$ SR Unit $=\mu \mathrm{G} / \mathrm{L}$

\begin{tabular}{|c|c|c|c|c|c|c|c|c|c|}
\hline Location & NM Date & EPA Date & $\begin{array}{l}\text { LANL } \\
\text { Date }\end{array}$ & $\begin{array}{l}\text { NMED } \\
\text { Result }\end{array}$ & $\begin{array}{l}\text { EPA } \\
\text { Result }\end{array}$ & $\begin{array}{l}\text { LANL } \\
\text { Result }\end{array}$ & $\begin{array}{l}\text { NMED vs } \\
\text { EPA }\end{array}$ & $\begin{array}{l}\text { NMED vs } \\
\text { LANL }\end{array}$ & $\begin{array}{l}\text { LANL vs } \\
\text { EPA }\end{array}$ \\
\hline $\mathrm{MCO}-3$ & $08 / 20 / 98$ & . & $08 / 20 / 98$ & 120 &. & 123 & - & $-2.46914^{*}$ & . \\
\hline MCO-5 & $08 / 20 / 98$ & & $05 / 27 / 98$ & 120 & . & 114 & & $5.12821^{*}$ & . \\
\hline
\end{tabular}

SUITE $=$ Metals Analyte $=$ URANIUM Unit $=\mu \mathrm{G} / \mathrm{L}$

\begin{tabular}{|c|c|c|c|c|c|c|c|c|c|}
\hline Location & NM Date & EPA Date & $\begin{array}{l}\text { LANL } \\
\text { Date }\end{array}$ & $\begin{array}{l}\text { NMED } \\
\text { Result }\end{array}$ & $\begin{array}{l}\text { EPA } \\
\text { Result }\end{array}$ & $\begin{array}{l}\text { LANL } \\
\text { Result }\end{array}$ & $\begin{array}{l}\text { NMED vs } \\
\text { EPA }\end{array}$ & $\begin{array}{l}\text { NMED vs } \\
\text { LANL }\end{array}$ & $\begin{array}{l}\text { LANL vs } \\
\text { EPA }\end{array}$ \\
\hline MCO-5 & $08 / 20 / 98$ & . & $05 / 27 / 98$ & 1.26 & . & 0.85125 & 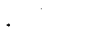 & $38.7211^{*}$ & . \\
\hline
\end{tabular}

Unfiltered

SUITE $=$ Organics Analyte $=1,1,1-$ TRICHLOROETHANE Unit $=\mu \mathrm{G} / \mathrm{L}$

\begin{tabular}{|c|c|c|c|c|c|c|c|c|c|}
\hline Location & NM Date & EPA Date & $\begin{array}{l}\text { LANL } \\
\text { Date }\end{array}$ & $\begin{array}{l}\text { NMED } \\
\text { Result }\end{array}$ & $\begin{array}{l}\text { EPA } \\
\text { Result }\end{array}$ & $\begin{array}{l}\text { LANL } \\
\text { Result }\end{array}$ & $\begin{array}{l}\text { NMED vs } \\
\text { EPA }\end{array}$ & $\begin{array}{l}\text { NMED vs } \\
\text { LANL }\end{array}$ & $\begin{array}{l}\text { LANL vs } \\
\text { EPA }\end{array}$ \\
\hline $\mathrm{MCO}-4 \mathrm{~B}$ & . & $08 / 20 / 98$ & $05 / 27 / 98$ & . & $<5$ & $<5$ & . & . & 0 \\
\hline MCO-5 & . & $08 / 20 / 98$ & $05 / 27 / 98$ & . & $<5$ & $<5$ & . & . & 0 \\
\hline
\end{tabular}

SUTTE $=$ Organics Analyte $=1,1,2,2-$ TETRACHLOROETHANE Unit $=\mu \mathrm{G} / \mathrm{L}$

\begin{tabular}{|c|c|c|c|c|c|c|c|c|c|}
\hline Location & NM Date & EPA Date & $\begin{array}{l}\text { LANL } \\
\text { Date }\end{array}$ & $\begin{array}{l}\text { NMED } \\
\text { Result }\end{array}$ & $\begin{array}{l}\text { EPA } \\
\text { Result }\end{array}$ & $\begin{array}{l}\text { LANL } \\
\text { Result }\end{array}$ & $\begin{array}{l}\text { NMED vs } \\
\text { EPA }\end{array}$ & $\begin{array}{l}\text { NMED vs } \\
\text { LANL }\end{array}$ & $\begin{array}{l}\text { LANL vs } \\
\text { EPA }\end{array}$ \\
\hline $\mathrm{MCO}-4 \mathrm{~B}$ & . & $08 / 20 / 98$ & $05 / 27 / 98$ & & $<5$ & $<5$ & . & . & 0 \\
\hline MCO-5 & . & $08 / 20 / 98$ & $05 / 27 / 98$ & . & $<5$ & $<5$ & 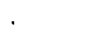 & . & 0 \\
\hline
\end{tabular}

- SUITE $=$ Organics Analyte $=1,1,2-$ TRICHLOROETHANE Unit $=\mu \mathrm{G} / \mathrm{L}$

\begin{tabular}{|c|c|c|c|c|c|c|c|c|c|}
\hline Location & NM Date & EPA Date & $\begin{array}{l}\text { LANL } \\
\text { Date }\end{array}$ & $\begin{array}{l}\text { NMED } \\
\text { Result }\end{array}$ & $\begin{array}{l}\text { EPA } \\
\text { Result }\end{array}$ & $\begin{array}{l}\text { LANL } \\
\text { Result }\end{array}$ & $\begin{array}{l}\text { NMED vs } \\
\text { EPA }\end{array}$ & $\begin{array}{l}\text { NMED vs } \\
\text { LANL }\end{array}$ & $\begin{array}{l}\text { LANL vs } \\
\text { EPA }\end{array}$ \\
\hline $\mathrm{MCO}-4 \mathrm{~B}$ & & $08 / 20 / 98$ & $05 / 27 / 98$ & . & $<5$ & $<5$ & $\theta^{\circ}$ & . & 0 \\
\hline MCO-5 & . & $08 / 20 / 98$ & $05 / 27 / 98$ & & $<5$ & $<5$ & . & . & 0 \\
\hline
\end{tabular}

SUITE $=$ Organics Analyte $=1,1-$ DICHLOROETHANE Unit $=\mu \mathrm{G} / \mathrm{L}$

\begin{tabular}{|c|c|c|c|c|c|c|c|c|c|}
\hline Location & NM Date & EPA Date & $\begin{array}{l}\text { LANL } \\
\text { Date }\end{array}$ & $\begin{array}{l}\text { NMED } \\
\text { Result }\end{array}$ & $\begin{array}{l}\text { EPA } \\
\text { Result }\end{array}$ & $\begin{array}{l}\text { LANL } \\
\text { Result }\end{array}$ & $\begin{array}{l}\text { NMED vs } \\
\text { EPA }\end{array}$ & $\begin{array}{l}\text { NMED vs } \\
\text { LANL }\end{array}$ & $\begin{array}{l}\text { LANL vs } \\
\text { EPA }\end{array}$ \\
\hline $\mathrm{MCO}-4 \mathrm{~B}$ & . & $08 / 20 / 98$ & $05 / 27 / 98$ & . & $<5$ & $<5$ & . &. & 0 \\
\hline MCO-5 & . & $08 / 20 / 98$ & $05 / 27 / 98$ & . & $<5$ & $<5$ & . & . & 0 \\
\hline
\end{tabular}

SUITE $=$ Organics Analyte $=1,1-$ DICHLOROETHENE Unit $=\mu \mathrm{G} /$

\begin{tabular}{|c|c|c|c|c|c|c|c|c|c|}
\hline Location & NM Date & EPA Date & LANL & NMED & EPA & LANL & NMED vs & NMED vs & LANL vs \\
\hline $\mathrm{MCO}-4 \mathrm{~B}$ & 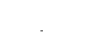 & & Date & Result & Result & Result & EPA & LANL & EPA \\
\hline $\begin{array}{l}\text { MCO-4B } \\
\text { MCO-5 }\end{array}$ & & $\begin{array}{l}08 / 20 / 98 \\
08 / 20 / 98\end{array}$ & $\begin{array}{l}05 / 27 / 98 \\
05 / 27 / 98\end{array}$ & : & $\begin{array}{l}<5 \\
<5\end{array}$ & $\begin{array}{l}<5 \\
<5\end{array}$ & . & . & $\begin{array}{l}0 \\
0\end{array}$ \\
\hline
\end{tabular}

SUTTE $=$ Organics Analyte $=1,2-$ DICHLOROETHANE Unit $=\mu \mathrm{G} / \mathrm{L}$

\begin{tabular}{|c|c|c|c|c|c|c|c|c|c|}
\hline Location & NM Date & EPA Date & $\begin{array}{l}\text { LANL } \\
\text { Date }\end{array}$ & $\begin{array}{l}\text { NMED } \\
\text { Result }\end{array}$ & $\begin{array}{l}\text { EPA } \\
\text { Result }\end{array}$ & $\begin{array}{l}\text { LANL } \\
\text { Result }\end{array}$ & $\begin{array}{l}\text { NMED vs } \\
\text { EPA }\end{array}$ & $\begin{array}{l}\text { NMED vs } \\
\text { LANL }\end{array}$ & $\begin{array}{l}\text { LANL vs } \\
\text { EPA }\end{array}$ \\
\hline MCO-4B & . & $08 / 20 / 98$ & $05 / 27 / 98$ & . & $<5$ & $<5$ & . & $\therefore$ & 0 \\
\hline MCO-5 & . & $08 / 20 / 98$ & $05 / 27 / 98$ & . & $<5$ & $<5$ & . & . & 0 \\
\hline
\end{tabular}


SUTTE $=$ Organics Analyte $=1,2$-DICHLOROETHENE Unit $=\mu \mathrm{G} / \mathrm{L}$

\begin{tabular}{|c|c|c|c|c|c|c|c|c|c|}
\hline Location & NM Date & EPA Date & $\begin{array}{l}\text { LANL } \\
\text { Date }\end{array}$ & $\begin{array}{l}\text { NMED } \\
\text { Result }\end{array}$ & $\begin{array}{l}\text { EPA } \\
\text { Result }\end{array}$ & $\begin{array}{l}\text { LANL } \\
\text { Result }\end{array}$ & $\begin{array}{l}\text { NMED vs } \\
\text { EPA }\end{array}$ & $\begin{array}{l}\text { NMED vs } \\
\text { LANL }\end{array}$ & $\begin{array}{l}\text { LANL vs } \\
\text { EPA }\end{array}$ \\
\hline $\mathrm{MCO}-4 \mathrm{~B}$ & . & $08 / 20 / 98$ & $05 / 27 / 98$ & . & $<5$ & $<5$ & . & & 0 \\
\hline MCO-5 & . & $08 / 20 / 98$ & $05 / 27 / 98$ & & $<5$ & $<5$ & . & & 0 \\
\hline
\end{tabular}

- SUTTE $=$ Organics Analyte $=1,2-$ DICHLOROPROPANE Unit $=\mu \mathrm{G} / \mathrm{L}$

\begin{tabular}{|c|c|c|c|c|c|c|c|c|c|}
\hline Location & NM Date & EPA Date & $\begin{array}{l}\text { LANL } \\
\text { Date }\end{array}$ & $\begin{array}{l}\text { NMED } \\
\text { Result }\end{array}$ & $\begin{array}{l}\text { EPA } \\
\text { Result }\end{array}$ & $\begin{array}{l}\text { LANL } \\
\text { Result }\end{array}$ & $\begin{array}{l}\text { NMED vs } \\
\text { EPA }\end{array}$ & $\begin{array}{l}\text { NMED vs } \\
\text { LANL }\end{array}$ & $\begin{array}{l}\text { LANL vs } \\
\text { EPA }\end{array}$ \\
\hline $\mathrm{MCO}-4 \mathrm{~B}$ & . & $08 / 20 / 98$ & $05 / 27 / 98$ & . & $<5$ & $<5$ & . & . & 0 \\
\hline $\mathrm{MCO}-5$ & . & $08 / 20 / 98$ & $05 / 27 / 98$ & . & $<5$ & $<5$ & . & . & 0 \\
\hline
\end{tabular}

- SUTTE $=$ Organics Analyte $=2-B U T A N O N E$ Unit $=\mu G / L$

\begin{tabular}{|c|c|c|c|c|c|c|c|c|c|}
\hline Location & NM Date & EPA Date & $\begin{array}{l}\text { LANL } \\
\text { Date }\end{array}$ & $\begin{array}{l}\text { NMED } \\
\text { Result }\end{array}$ & $\begin{array}{l}\text { EPA } \\
\text { Result }\end{array}$ & $\begin{array}{l}\text { LANL } \\
\text { Result }\end{array}$ & $\begin{array}{l}\text { NMED vs } \\
\text { EPA }\end{array}$ & $\begin{array}{l}\text { NMED vs } \\
\text { LANL }\end{array}$ & $\begin{array}{l}\text { LANL vs } \\
\text { EPA }\end{array}$ \\
\hline $\mathrm{MCO}-4 \mathrm{~B}$ & . & $08 / 20 / 98$ & $05 / 27 / 98$ & . & $<20$ & $<20$ & . & . & 0 \\
\hline MCO-5 & . & $08 / 20 / 98$ & $05 / 27 / 98$ & . & $<20$ & $<20$ & . & . & 0 \\
\hline
\end{tabular}

SUITE $=$ Organics Analyte $=2-$ HEXANONE Unit $=\mu \mathrm{G} / \mathrm{L}$

\begin{tabular}{|c|c|c|c|c|c|c|c|c|c|}
\hline Location & NM Date & EPA Date & $\begin{array}{l}\text { LANL } \\
\text { Date }\end{array}$ & $\begin{array}{l}\text { NMED } \\
\text { Result }\end{array}$ & $\begin{array}{l}\text { EPA } \\
\text { Result }\end{array}$ & $\begin{array}{l}\text { LANL } \\
\text { Result }\end{array}$ & $\begin{array}{l}\text { NMED vs } \\
\text { EPA }\end{array}$ & $\begin{array}{l}\text { NMED vs } \\
\text { LANL }\end{array}$ & $\begin{array}{l}\text { LANL vs } \\
\text { EPA }\end{array}$ \\
\hline $\mathrm{MCO}-4 \mathrm{~B}$ & . & $08 / 20 / 98$ & $05 / 27 / 98$ & & $<20$ & $<20$ & . & . & 0 \\
\hline MCO-5 & . & $08 / 20 / 98$ & $05 / 27 / 98$ & & $<20$ & $<20$ & . & 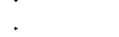 & 0 \\
\hline
\end{tabular}

SUTTE $=$ Organics Analyte $=4-\mathrm{METHYL}-2-\mathrm{PENTANONE} U_{\text {nit }}=\mu \mathrm{G} / \mathrm{L}$

\begin{tabular}{|c|c|c|c|c|c|c|c|c|c|}
\hline Location & NM Date & EPA Date & $\begin{array}{l}\text { LANL } \\
\text { Date }\end{array}$ & $\begin{array}{l}\text { NMED } \\
\text { Result }\end{array}$ & $\begin{array}{l}\text { EPA } \\
\text { Result }\end{array}$ & $\begin{array}{l}\text { LANL } \\
\text { Result }\end{array}$ & $\begin{array}{l}\text { NMED vs } \\
\text { EPA }\end{array}$ & $\begin{array}{l}\text { NMED vs } \\
\text { LANL }\end{array}$ & $\begin{array}{l}\text { LANL vs } \\
\text { EPA }\end{array}$ \\
\hline $\mathrm{MCO}-4 \mathrm{~B}$ & . & $08 / 20 / 98$ & $05 / 27 / 98$ & . & $<20$ & $<20$ & . & . & 0 \\
\hline MCO-5 & . & $08 / 20 / 98$ & $05 / 27 / 98$ & . & $<20$ & $<20$ & . & . & 0 \\
\hline
\end{tabular}

SUTTE $=$ Organics Analyte $=$ ACETONE Unit $=\mu \mathrm{G} / \mathrm{L}$

\begin{tabular}{|c|c|c|c|c|c|c|c|c|c|}
\hline Location & NM Date & EPA Date & $\begin{array}{l}\text { LANL } \\
\text { Date }\end{array}$ & $\begin{array}{l}\text { NMED } \\
\text { Result }\end{array}$ & $\begin{array}{l}\text { EPA } \\
\text { Result }\end{array}$ & $\begin{array}{l}\text { LANL } \\
\text { Result }\end{array}$ & $\begin{array}{l}\text { NMED vs } \\
\text { EPA }\end{array}$ & $\begin{array}{l}\text { NMED vs } \\
\text { LANL }\end{array}$ & $\begin{array}{l}\text { LANL vs } \\
\text { EPA }\end{array}$ \\
\hline $\mathrm{MCO}-4 \mathrm{~B}$ & . & $08 / 20 / 98$ & $05 / 27 / 98$ & & 12 & 86 & . & . & $151.020^{*}$ \\
\hline MCO-5 & . & $08 / 20 / 98$ & $05 / 27 / 98$ & & $<20$ & 146 & . & . & 151.807 \\
\hline
\end{tabular}

Unfiltered

SUTTE $=$ Organics Analyte $=$ BENZENE Unit $=\mu \mathrm{G} / \mathrm{L}$

\begin{tabular}{|c|c|c|c|c|c|c|c|c|c|}
\hline Location & NM Date & EPA Date & $\begin{array}{l}\text { LANL } \\
\text { Date }\end{array}$ & $\begin{array}{l}\text { NMED } \\
\text { Result }\end{array}$ & $\begin{array}{l}\text { EPA } \\
\text { Result }\end{array}$ & $\begin{array}{l}\text { LANL } \\
\text { Result }\end{array}$ & $\begin{array}{l}\text { NMED vs } \\
\text { EPA }\end{array}$ & $\begin{array}{l}\text { NMED vs } \\
\text { LANL }\end{array}$ & $\begin{array}{l}\text { LANL vs } \\
\text { EPA }\end{array}$ \\
\hline $\mathrm{MCO}-4 \mathrm{~B}$ & . & $08 / 20 / 98$ & $05 / 27 / 98$ & . & $<5$ & $<5$ & . & . & 0 \\
\hline MCO-5 & . & $08 / 20 / 98$ & $05 / 27 / 98$ & . & $<5$ & $<5$ & . & . & 0 \\
\hline
\end{tabular}

- SUTTE $=$ Organics Analyte $=$ BROMODICHLOROMETHANE Unit $=\mu \mathrm{G} / \mathrm{L}$

\begin{tabular}{|c|c|c|c|c|c|c|c|c|c|}
\hline Location & NM Date & EPA Date & $\begin{array}{l}\text { LANL } \\
\text { Date }\end{array}$ & $\begin{array}{l}\text { NMED } \\
\text { Result }\end{array}$ & $\begin{array}{l}\text { EPA } \\
\text { Result }\end{array}$ & $\begin{array}{l}\text { LANL } \\
\text { Result }\end{array}$ & $\begin{array}{l}\text { NMED vs } \\
\text { EPA }\end{array}$ & $\begin{array}{l}\text { NMED vs } \\
\text { LANL }\end{array}$ & $\begin{array}{l}\text { LANL vs } \\
\text { EPA }\end{array}$ \\
\hline MCO-4B & . & $08 / 20 / 98$ & $05 / 27 / 98$ & . & $<5$ & $<5$ & 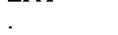 & . & 0 \\
\hline MCO-5 & . & $08 / 20 / 98$ & $05 / 27 / 98$ & . & $<5$ & $<5$ & . & : & 0 \\
\hline
\end{tabular}

SUTTE $=$ Organics Analyte $=$ BROMOFORM Unit $=\mu \mathrm{G} / \mathrm{L}$

\begin{tabular}{|c|c|c|c|c|c|c|c|c|c|}
\hline Location & NM Date & EPA Date & $\begin{array}{l}\text { LANL } \\
\text { Date }\end{array}$ & $\begin{array}{l}\text { NMED } \\
\text { Result }\end{array}$ & $\begin{array}{l}\text { EPA } \\
\text { Result }\end{array}$ & $\begin{array}{l}\text { LANL } \\
\text { Result }\end{array}$ & $\begin{array}{l}\text { NMED vs } \\
\text { EPA }\end{array}$ & $\begin{array}{l}\text { NMED vs } \\
\text { LANL }\end{array}$ & $\begin{array}{l}\text { LANL vs } \\
\text { EPA }\end{array}$ \\
\hline $\mathrm{MCO}-4 \mathrm{~B}$ & . & $08 / 20 / 98$ & $05 / 27 / 98$ & . & $<5$ & $<5$ & . & . & 0 \\
\hline MCO-5 & . & $08 / 20 / 98$ & $05 / 27 / 98$ & . & $<5$ & $<5$ & . & . & 0 \\
\hline
\end{tabular}

SUITE $=$ Organics Analyte $=$ BROMOMETHANE Unit $=\mu \mathrm{G} / \mathrm{L}$

\begin{tabular}{|c|c|c|c|c|c|c|c|c|c|}
\hline Location & NM Date & EPA Date & $\begin{array}{l}\text { LANL } \\
\text { Date }\end{array}$ & $\begin{array}{l}\text { NMED } \\
\text { Result }\end{array}$ & $\begin{array}{l}\text { EPA } \\
\text { Result }\end{array}$ & $\begin{array}{l}\text { LANL } \\
\text { Result }\end{array}$ & $\begin{array}{l}\text { NMED vs } \\
\text { EPA }\end{array}$ & $\begin{array}{l}\text { NMED vs } \\
\text { LANL }\end{array}$ & $\begin{array}{l}\text { LANL vs } \\
\text { EPA }\end{array}$ \\
\hline MCO-4B & . & $08 / 20 / 98$ & $05 / 27 / 98$ & . & $<10$ & $<10$ & . & . & 0 \\
\hline MCO-5 & . & $08 / 20 / 98$ & $05 / 27 / 98$ & & $<10$ & $<10$ & . & 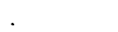 & 0 \\
\hline
\end{tabular}

SUITE $=$ Organics Analyte $=$ CARBON DISULFIDE Unit $=\mu G / L$

\begin{tabular}{|c|c|c|c|c|c|c|c|c|c|}
\hline Location & NM Date & EPA Date & $\begin{array}{l}\text { LANL } \\
\text { Date }\end{array}$ & $\begin{array}{l}\text { NMED } \\
\text { Result }\end{array}$ & $\begin{array}{l}\text { EPA } \\
\text { Result }\end{array}$ & $\begin{array}{l}\text { LANL } \\
\text { Result }\end{array}$ & $\begin{array}{l}\text { NMED vs } \\
\text { EPA }\end{array}$ & $\begin{array}{l}\text { NMED vs } \\
\text { LANL }\end{array}$ & $\begin{array}{l}\text { LANL vs } \\
\text { EPA }\end{array}$ \\
\hline $\mathrm{MCO}-4 \mathrm{~B}$ & . & $08 / 20 / 98$ & $05 / 27 / 98$ & . & $<5$ & $<5$ & . & . & 0 \\
\hline $\mathrm{MCO}-5$ & . & $08 / 20 / 98$ & $05 / 27 / 98$ & . & $<5$ & $<5$ & . & . & 0 \\
\hline
\end{tabular}


- SUITE $=$ Organics Analyte $=$ CHLOROBENZENE Unit $=\mu \mathrm{G} / \mathrm{L}$

\begin{tabular}{|c|c|c|c|c|c|c|c|c|c|}
\hline Location & NM Date & EPA Date & $\begin{array}{l}\text { LANL } \\
\text { Date }\end{array}$ & $\begin{array}{l}\text { NMED } \\
\text { Result }\end{array}$ & $\begin{array}{l}\text { EPA } \\
\text { Result }\end{array}$ & $\begin{array}{l}\text { LANL } \\
\text { Result }\end{array}$ & $\begin{array}{l}\text { NMED vs } \\
\text { EPA }\end{array}$ & $\begin{array}{l}\text { NMED vs } \\
\text { LANL }\end{array}$ & $\begin{array}{l}\text { LANL vs } \\
\text { EPA }\end{array}$ \\
\hline $\mathrm{MCO}-4 \mathrm{~B}$ & - & $08 / 20 / 98$ & $05 / 27 / 98$ & . & $<5$ & $<5$ & . & . & 0 \\
\hline MCO-5 & . & $08 / 20 / 98$ & $05 / 27 / 98$ & . & $<5$ & $<5$ & 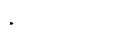 & . & 0 \\
\hline
\end{tabular}

SUTTE $=$ Organics Analyte $=$ CHLORODIBROMOMETHANE Unit $=\mu \mathrm{G} / \mathrm{L}$

\begin{tabular}{|c|c|c|c|c|c|c|c|c|c|}
\hline Location & NM Date & EPA Date & $\begin{array}{l}\text { LANL } \\
\text { Date }\end{array}$ & $\begin{array}{l}\text { NMED } \\
\text { Result }\end{array}$ & $\begin{array}{l}\text { EPA } \\
\text { Result }\end{array}$ & $\begin{array}{l}\text { LANL } \\
\text { Result }\end{array}$ & $\begin{array}{l}\text { NMED vs } \\
\text { EPA }\end{array}$ & $\begin{array}{l}\text { NMED vs } \\
\text { LANL }\end{array}$ & $\begin{array}{l}\text { LANL vs } \\
\text { EPA }\end{array}$ \\
\hline $\mathrm{MCO}-4 \mathrm{~B}$ & . & $08 / 20 / 98$ & $05 / 27 / 98$ & . & $<5$ & $<5$ & . & . & 0 \\
\hline MCO-5 & . & $08 / 20 / 98$ & $05 / 27 / 98$ & . & $<5$ & $<5$ & . & - & 0 \\
\hline
\end{tabular}

SUITE $=$ Organics Analyte $=$ CHLOROETHANE Unit $=\mu$ G/L

\begin{tabular}{|c|c|c|c|c|c|c|c|c|c|}
\hline Location & NM Date & EPA Date & $\begin{array}{l}\text { LANL } \\
\text { Date }\end{array}$ & $\begin{array}{l}\text { NMED } \\
\text { Result }\end{array}$ & $\begin{array}{l}\text { EPA } \\
\text { Result }\end{array}$ & $\begin{array}{l}\text { LANL } \\
\text { Result }\end{array}$ & $\begin{array}{l}\text { NMED vs } \\
\text { EPA }\end{array}$ & $\begin{array}{l}\text { NMED vs } \\
\text { LANL }\end{array}$ & $\begin{array}{l}\text { LANL vs } \\
\text { EPA }\end{array}$ \\
\hline $\mathrm{MCO}-4 \mathrm{~B}$ & . & $08 / 20 / 98$ & $05 / 27 / 98$ & . & $<10$ & $<10$ & . & & 0 \\
\hline MCO-5 & . & $08 / 20 / 98$ & $05 / 27 / 98$ & . & $<10$ & $<10$ & & & 0 \\
\hline
\end{tabular}

SUTTE $=$ Organics Analyte $=$ CHLOROFORM Unit $=\mu \mathrm{G} / \mathrm{L}$

\begin{tabular}{|c|c|c|c|c|c|c|c|c|c|}
\hline Location & NM Date & EPA Date & $\begin{array}{l}\text { LANL } \\
\text { Date }\end{array}$ & $\begin{array}{l}\text { NMED } \\
\text { Result }\end{array}$ & $\begin{array}{l}\text { EPA } \\
\text { Result }\end{array}$ & $\begin{array}{l}\text { LANL } \\
\text { Result }\end{array}$ & $\begin{array}{l}\text { NMED vs } \\
\text { EPA }\end{array}$ & $\begin{array}{l}\text { NMED vs } \\
\text { LANL }\end{array}$ & $\begin{array}{l}\text { LANL vs } \\
\text { EPA }\end{array}$ \\
\hline $\mathrm{MCO}-4 \mathrm{~B}$ & . & $08 / 20 / 98$ & $05 / 27 / 98$ & . & $<5$ & $<5$ & . & , & 0 \\
\hline MCO-5 & . & $08 / 20 / 98$ & $05 / 27 / 98$ & . & $<5$ & $<5$ & . & . & 0 \\
\hline
\end{tabular}

SUITE $=$ Organics Analyte $=$ CHLOROMETHANE Unit $=\mu G / L$

\begin{tabular}{|c|c|c|c|c|c|c|c|c|c|}
\hline Location & NM Date & EPA Date & $\begin{array}{l}\text { LANL } \\
\text { Date }\end{array}$ & $\begin{array}{l}\text { NMED } \\
\text { Result }\end{array}$ & $\begin{array}{l}\text { EPA } \\
\text { Result }\end{array}$ & $\begin{array}{l}\text { LANL } \\
\text { Result }\end{array}$ & $\begin{array}{l}\text { NMED vs } \\
\text { EPA }\end{array}$ & $\begin{array}{l}\text { NMED vs } \\
\text { LANL }\end{array}$ & $\begin{array}{l}\text { LANL vs } \\
\text { EPA }\end{array}$ \\
\hline $\mathrm{MCO}-4 \mathrm{~B}$ & . & $08 / 20 / 98$ & $05 / 27 / 98$ & . & $<10$ & $<10$ & . & . & 0 \\
\hline MCO-5 & $\therefore$ & $08 / 20 / 98$ & $05 / 27 / 98$ & . & $<10$ & $<10$ & . & . & 0 \\
\hline
\end{tabular}

SUITE $=$ Organics Analyte $=$ CIS-1,3-DICHLOROPROPENE Unit $=\mu \mathrm{G} / \mathrm{L}$

\begin{tabular}{|c|c|c|c|c|c|c|c|c|c|}
\hline Location & NM Date & EPA Date & $\begin{array}{l}\text { LANL } \\
\text { Date }\end{array}$ & $\begin{array}{l}\text { NMED } \\
\text { Result }\end{array}$ & $\begin{array}{l}\text { EPA } \\
\text { Result }\end{array}$ & $\begin{array}{l}\text { LANL } \\
\text { Result }\end{array}$ & $\begin{array}{l}\text { NMED vs } \\
\text { EPA }\end{array}$ & $\begin{array}{l}\text { NMED vs } \\
\text { LANL }\end{array}$ & $\begin{array}{l}\text { LANL vs } \\
\text { EPA }\end{array}$ \\
\hline $\mathrm{MCO}-4 \mathrm{~B}$ & . & $08 / 20 / 98$ & $05 / 27 / 98$ & . & $<$ & $<5$ & . & . & 0 \\
\hline MCO-5 & . & $08 / 20 / 98$ & $05 / 27 / 98$ & . & $<5$ & $<5$ & . & . & 0 \\
\hline
\end{tabular}

SUITE $=$ Organics Analyte $=$ METHYLENE CHLORIDE Unit $=\mu \mathrm{G} / \mathrm{L}$

\begin{tabular}{|c|c|c|c|c|c|c|c|c|c|}
\hline Location & NM Date & EPA Date & $\begin{array}{l}\text { LANL } \\
\text { Date }\end{array}$ & $\begin{array}{l}\text { NMED } \\
\text { Result }\end{array}$ & $\begin{array}{l}\text { EPA } \\
\text { Result }\end{array}$ & $\begin{array}{l}\text { LANL } \\
\text { Result }\end{array}$ & $\begin{array}{l}\text { NMED vs } \\
\text { EPA }\end{array}$ & $\begin{array}{l}\text { NMED vs } \\
\text { LANL }\end{array}$ & $\begin{array}{l}\text { LANL vs } \\
\text { EPA }\end{array}$ \\
\hline $\mathrm{MCO}-4 \mathrm{~B}$ & . & $08 / 20 / 98$ & $05 / 27 / 98$ & . & $<5$ & $<5$ & . & 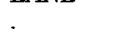 & 0 \\
\hline $\mathrm{MCO}-5$ & . & $08 / 20 / 98$ & $05 / 27 / 98$ & . & $\delta$ & $<5$ & . & . & 0 \\
\hline
\end{tabular}

Unfiltered

SUTTE $=$ Organics Analyte $=$ STYRENE Unit $=\mu \mathrm{G} / \mathrm{L}$

\begin{tabular}{|c|c|c|c|c|c|c|c|c|c|}
\hline Location & NM Date & EPA Date & $\begin{array}{l}\text { LANL } \\
\text { Date }\end{array}$ & $\begin{array}{l}\text { NMED } \\
\text { Result }\end{array}$ & $\begin{array}{l}\text { EPA } \\
\text { Result }\end{array}$ & $\begin{array}{l}\text { LANL } \\
\text { Result }\end{array}$ & $\begin{array}{l}\text { NMED vs } \\
\text { EPA }\end{array}$ & $\begin{array}{l}\text { NMED vs } \\
\text { LANL }\end{array}$ & $\begin{array}{l}\text { LANL vs } \\
\text { EPA }\end{array}$ \\
\hline $\mathrm{MCO}-4 \mathrm{~B}$ & . & $08 / 20 / 98$ & $05 / 27 / 98$ & . & $<5$ & $<5$ & . & . & 0 \\
\hline MCO-5 & . & $08 / 20 / 98$ & $05 / 27 / 98$ & . & 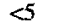 & $<5$ & . & . & 0 \\
\hline
\end{tabular}

SUITE $=$ Organics Analyte $=$ TETRACHLOROETHENE Unit $=\mu \mathrm{G} / \mathrm{L}$

\begin{tabular}{|c|c|c|c|c|c|c|c|c|c|}
\hline Location & NM Date & EPA Date & $\begin{array}{l}\text { LANL } \\
\text { Date }\end{array}$ & $\begin{array}{l}\text { NMED } \\
\text { Result }\end{array}$ & $\begin{array}{l}\text { EPA } \\
\text { Result }\end{array}$ & $\begin{array}{l}\text { LANL } \\
\text { Result }\end{array}$ & $\begin{array}{l}\text { NMED vs } \\
\text { EPA }\end{array}$ & $\begin{array}{l}\text { NMED vs } \\
\text { LANL }\end{array}$ & $\begin{array}{l}\text { LANL vs } \\
\text { EPA }\end{array}$ \\
\hline $\mathrm{MCO}-4 \mathrm{~B}$ & . & $08 / 20 / 98$ & $05 / 27 / 98$ & $\theta^{\circ}$ & $<5$ & $<5$ & . & . & 0 \\
\hline MCO-5 & . & $08 / 20 / 98$ & $05 / 27 / 98$ & . & $<5$ & $<5$ & . & . & 0 \\
\hline
\end{tabular}

- SUITE $=$ Organics Analyte $=$ TOLUENE Unit $=\mu \mathrm{G} / \mathrm{L}$

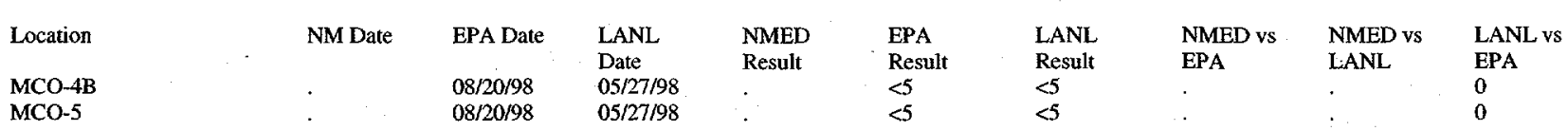

SUITE $=$ Organics Analyte $=$ TRICHLOROETHENE Unit $=\mu \mathrm{G} / \mathrm{L}$

\begin{tabular}{|c|c|c|c|c|c|c|c|c|c|}
\hline Location & NM Date & EPA Date & $\begin{array}{l}\text { LANL } \\
\text { Date }\end{array}$ & $\begin{array}{l}\text { NMED } \\
\text { Result }\end{array}$ & $\begin{array}{l}\text { EPA } \\
\text { Result }\end{array}$ & $\begin{array}{l}\text { LANL } \\
\text { Result }\end{array}$ & $\begin{array}{l}\text { NMED vs } \\
\text { EPA }\end{array}$ & $\begin{array}{l}\text { NMED vs } \\
\text { LANL }\end{array}$ & $\begin{array}{l}\text { LANL vs } \\
\text { EPA }\end{array}$ \\
\hline MCO-4B & 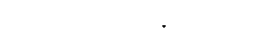 & $08 / 20 / 98$ & $05 / 27 / 98$ & & $<5$ & $<5$ & . & . & 0 \\
\hline $\mathrm{MCO}-5$ & . & $08 / 20 / 98$ & $05 / 27 / 98$ & & $<5$ & $<5$ & . & . & 0 \\
\hline
\end{tabular}


SUITE $=$ Organics Analyte $=$ VINYL CHLORIDE Unit $=\mu \mathrm{G} / \mathrm{L}$

\begin{tabular}{|c|c|c|c|c|c|c|c|c|c|}
\hline Location & NM Date & EPA Date & $\begin{array}{l}\text { LANL } \\
\text { Date }\end{array}$ & $\begin{array}{l}\text { NMED } \\
\text { Result }\end{array}$ & $\begin{array}{l}\text { EPA } \\
\text { Result }\end{array}$ & $\begin{array}{l}\text { LANL } \\
\text { Result }\end{array}$ & $\begin{array}{l}\text { NMED vs } \\
\text { EPA }\end{array}$ & $\begin{array}{l}\text { NMED vs } \\
\text { LANL }\end{array}$ & $\begin{array}{l}\text { LANL vs } \\
\text { EPA }\end{array}$ \\
\hline $\mathrm{MCO}-4 \mathrm{~B}$ & . & $08 / 20 / 98$ & $05 / 27 / 98$ & . & $<10$ & $<10$ & . & . & 0 \\
\hline MCO-5 & . & $08 / 20 / 98$ & $05 / 27 / 98$ & . & $<10$ & $<10$ & . & . & 0 \\
\hline
\end{tabular}

SUITE $=$ Rads Analyte $=$ AM-241 Unit $=$ PCI $/ L$

\begin{tabular}{|c|c|c|c|c|c|c|c|c|c|}
\hline Location & NM Date & EPA Date & $\begin{array}{l}\text { LANL } \\
\text { Date }\end{array}$ & $\begin{array}{l}\text { NMED } \\
\text { Result }\end{array}$ & $\begin{array}{l}\text { EPA } \\
\text { Result }\end{array}$ & $\begin{array}{l}\text { LANL } \\
\text { Result }\end{array}$ & $\begin{array}{l}\text { NMED vs } \\
\text { EPA }\end{array}$ & $\begin{array}{l}\text { NMED vs } \\
\text { LANL }\end{array}$ & $\begin{array}{l}\text { LANL vs } \\
\text { EPA }\end{array}$ \\
\hline $\mathrm{MCO}-3$ & $08 / 20 / 98$ & & $08 / 20 / 98$ & 0.425 & . & 0.4980 & . & $-15.818^{*}$ & . \\
\hline MCO-5 & $08 / 20 / 98$ & . & $05 / 27 / 98$ & 1.100 & . & 0.9067 & . & $19.265^{*}$ & . \\
\hline MORTANDAD AT GS-1 & $08 / 28 / 98$ & . & $08 / 28 / 98$ & 4.200 & . & 24.9014 & . & $-142.271^{*}$ & . \\
\hline TW-8 & $09 / 02 / 98$ & . & $09 / 02 / 98$ & 0.028 & . & 0.0215 & . & $26.263 *$ & . \\
\hline
\end{tabular}

SUITE $=$ Rads Analyte $=$ GROSS ALPHA Unit $=$ PCI/L

\begin{tabular}{|c|c|c|c|c|c|c|c|c|c|}
\hline Location & NM Date & EPA Date & $\begin{array}{l}\text { LANL } \\
\text { Date }\end{array}$ & $\begin{array}{l}\text { NMED } \\
\text { Result }\end{array}$ & $\begin{array}{l}\text { EPA } \\
\text { Result }\end{array}$ & $\begin{array}{l}\text { LANL } \\
\text { Result }\end{array}$ & $\begin{array}{l}\text { NMED vs } \\
\text { EPA }\end{array}$ & $\begin{array}{l}\text { NMED vs } \\
\text { LANL }\end{array}$ & $\begin{array}{l}\text { LANL vs } \\
\text { EPA }\end{array}$ \\
\hline APCO-1 & & $09 / 03 / 98$ & $09 / 03 / 98$ & & 4.200 & 0.723 & & . & $-141.273^{*}$ \\
\hline BAYO-1 (OUTFALL) & $09 / 01 / 98$ & $09 / 01 / 98$ & . & $<2.2$ & 3.390 & . & -42.5760 & . & . \\
\hline LAO-2 & . & $08 / 31 / 98$ & $08 / 31 / 98$ & . & 3.420 & -0.327 & . & . & $-242.325^{*}$ \\
\hline $\mathrm{LAO}-3 \mathrm{~A}$ & . & $08 / 31 / 98$ & $08 / 31 / 98$ & . & 11.200 & -0.717 & . & . & $-227.369 *$ \\
\hline $\mathrm{LAO}-4.5 \mathrm{C}$ & . & $08 / 31 / 98$ & $05 / 14 / 98$ & . & 2.860 & 0.613 & . & . & $-129.422 *$ \\
\hline $\mathrm{MCO}-3$ & . & $08 / 20 / 98$ & $08 / 20 / 98$ & . & 19.700 & 8.093 & . & . & $-83.527^{*}$ \\
\hline $\mathrm{MCO}-4 \mathrm{~B}$ & . & $08 / 20 / 98$ & $05 / 27 / 98$ & . & 12.700 & -5.027 & . & . & $-462.083^{*}$ \\
\hline $\mathrm{MCO}-5$ & . & $08 / 20 / 98$ & $05 / 27 / 98$ & . & 15.100 & -1.567 & . & . & $-246.325^{*}$ \\
\hline MORTANDAD AT GS-1 & $08 / 28 / 98$ & & $08 / 28 / 98$ & 20.9 & . & 200.413 & . & $-162.225 *$ & \\
\hline MT-3 & . & $08 / 27 / 98$ & $09 / 04 / 98$ & . & 5.520 & 1.483 & . & . & $-115.305^{*}$ \\
\hline MT-4 & & $08 / 26 / 98$ & $05 / 14 / 98$ & . & 4.620 & -0.747 & . & . & $-277.180^{*}$ \\
\hline TA-50 OUTFALL & $08 / 28 / 98$ & $08 / 28 / 98$ & . & 160.0 & 133.000 & & $18.4300^{*}$ & . & . \\
\hline TW-1 & . & $09 / 01 / 98$ & $05 / 28 / 98$ & . & 6.160 & 1.973 & . & . & $-102.973^{*}$ \\
\hline$T W-2 A$ & & $09 / 01 / 98$ & $09 / 01 / 98$ & . & 7.960 & -0.837 & . & . & $-247.018^{*}$ \\
\hline TW-3 & & $09 / 01 / 98$ & $09 / 01 / 98$ & . & 1.620 & 0.073 & . & . & $-182.809 *$ \\
\hline TW-4 & & $09 / 01 / 98$ & $09 / 01 / 98$ & . & 1.840 & 0.323 & . & . & $-140.307 *$ \\
\hline TW-8 & & $09 / 02 / 98$ & $09 / 02 / 98$ & . & 1.070 & 0.573 & . & . & $-60.539^{*}$ \\
\hline
\end{tabular}

- SUTTE $=$ Rads Analyte $=$ GROSS BETA Unit $=$ PCI/L

\begin{tabular}{|c|c|c|c|c|c|c|c|c|c|}
\hline Location & NM Date & EPA Date & $\begin{array}{l}\text { LANL } \\
\text { Date }\end{array}$ & $\begin{array}{l}\text { NMED } \\
\text { Result }\end{array}$ & $\begin{array}{l}\text { EPA } \\
\text { Result }\end{array}$ & $\begin{array}{l}\text { LANL } \\
\text { Result }\end{array}$ & $\begin{array}{l}\text { NMED vs } \\
\text { EPA }\end{array}$ & $\begin{array}{l}\text { NMED vs } \\
\text { LANL }\end{array}$ & $\begin{array}{l}\text { LANL vs } \\
\text { EPA }\end{array}$ \\
\hline APCO-1 & & $09 / 03 / 98$ & 09/03/98 & . & 12.000 & 9.007 & & . & $-28.490 *$ \\
\hline BAYO-1 (OUTFALL) & $09 / 01 / 98$ & $09 / 01 / 98$ & . & 14.2 & 13.200 & . & $7.2993^{*}$ & . & . \\
\hline $\mathrm{LAO}-2$ & . & $08 / 31 / 98$ & $08 / 31 / 98$ & . & 37.100 & 30.517 & . & . & $-19.470^{*}$ \\
\hline LAO-3A & & $08 / 31 / 98$ & $08 / 31 / 98$ & . & 80.400 & 72.517 & . & . & $-10.309^{*}$ \\
\hline $\mathrm{LAO}-4.5 \mathrm{C}$ & . & $08 / 31 / 98$ & $05 / 14 / 98$ & . & 9.710 & 5.807 & . & . & $-50.298^{*}$ \\
\hline MCO-3 & . & $08 / 20 / 98$ & $08 / 20 / 98$ & . & 148.000 & 89.417 & . & . & $-49.350^{*}$ \\
\hline $\mathrm{MCO}-4 \mathrm{~B}$ & . & $08 / 20 / 98$ & $05 / 27 / 98$ & . & 118.000 & 264.318 & . & . & $76.542 *$ \\
\hline MORTANDAD AT GS-1 & $08 / 28 / 98$ & & $08 / 28 / 98$ & 479.0 & & 294.318 & . & $47.7637 *$ & r -5 \\
\hline MT-3 & . & $08 / 27 / 98$ & $09 / 04 / 98$ & . & 32.700 & 27.217 & . & . & $-18.300^{*}$ \\
\hline MT-4 & & $08 / 26 / 98$ & $05 / 14 / 98$ & & 35.800 & 25.617 & . & . & $-33.158^{*}$ \\
\hline TA-50 OUTFALL & $08 / 28 / 98$ & $08 / 28 / 98$ & . & 391.0 & 489.000 & . & $-22.2727^{*}$ & . & . \\
\hline TW-1 & . & $09 / 01 / 98$ & $05 / 28 / 98$ & . & 13.900 & 4.328 & $\cdot$ & . & $-105.034^{*}$ \\
\hline TW-2A & . & $09 / 01 / 98$ & 09/01/98 & . & 4.720 & 1.288 & . & . & $-114.274^{*}$ \\
\hline TW-3 & . & $09 / 01 / 98$ & $09 / 01 / 98$ & . & 4.600 & 2.148 & . & . & $-72.694^{*}$ \\
\hline TW-4 & 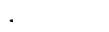 & $09 / 01 / 98$ & 09/01/98 & . & 4.020 & 2.008 & . & . & $-66.777 *$ \\
\hline TW-8 & . & $09 / 02 / 98$ & $09 / 02 / 98$ & $\cdot$ & 2.28000 & 1.70750 & . & . & $-28.7147^{*}$ \\
\hline
\end{tabular}

Unfiltered

- SUTTE $=$ Rads Analyte $=\mathrm{H}-3$ Unit $=$ PCI $/$

\begin{tabular}{|c|c|c|c|c|c|c|c|c|c|}
\hline Location & NM Date & EPA Date & $\begin{array}{l}\text { LANL } \\
\text { Date }\end{array}$ & $\begin{array}{l}\text { NMED } \\
\text { Result }\end{array}$ & $\begin{array}{l}\text { EPA } \\
\text { Result }\end{array}$ & $\begin{array}{l}\text { LANL } \\
\text { Result }\end{array}$ & $\begin{array}{l}\text { NMED vs } \\
\text { EPA }\end{array}$ & $\begin{array}{l}\text { NMED vs } \\
\text { LANL }\end{array}$ & $\begin{array}{l}\text { LANL vs } \\
\text { EPA }\end{array}$ \\
\hline APCO-1 & r. & $09 / 03 / 98$ & $09 / 03 / 98$ & . & 31.20 & 521.25 & . & . & $177.410^{*}$ \\
\hline BASALT SPRING & $\because$ & $09 / 09 / 98$ & $06 / 04 / 98$ & . & 42.10 & 21.25 & . & . & $-65.825^{*}$ \\
\hline DP SPRING & . & $09 / 02 / 98$ & $09 / 02 / 98$ & . & 156.00 & 361.25 & . & . & $79.362^{*}$ \\
\hline LA MESITA SPRING & . & $09 / 08 / 98$ & $09 / 08 / 98$ & . & 54.50 & 21.25 & . & . & $-87.789^{*}$ \\
\hline LAO-2 & . & $08 / 31 / 98$ & $08 / 31 / 98$ & . & 134.00 & 461.25 & . & . & $109.954^{*}$ \\
\hline LAO-3A & . & $08 / 31 / 98$ & $08 / 31 / 98$ & . & 99.80 & 351.25 & . & . & $111.495^{*}$ \\
\hline $\mathrm{LAO}-4.5 \mathrm{C}$ & . & $08 / 31 / 98$ & $05 / 14 / 98$ & 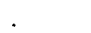 & 169.00 & 221.25 & . & . & $26.778^{*}$ \\
\hline $\mathrm{MCO}-2$ & $08 / 19 / 98$ & $08 / 19 / 98$ & . & 11 & 105.00 & & $-162.069^{*}$ & & \\
\hline $\mathrm{MCO}-3$ & $08 / 20 / 98$ & $08 / 20 / 98$ & $08 / 20 / 98$ & 19900 & 19200.00 & 19211.25 & $3.581 *$ & $3.5220^{*}$ & $0.059^{*}$ \\
\hline MCO-4B & & $08 / 20 / 98$ & $05 / 27 / 98$ & & 8720.00 & 12711.25 & & & $37.247 *$ \\
\hline MCO-5 & $08 / 20 / 98$ & $08 / 20 / 98$ & $05 / 27 / 98$ & 11800 & 11600.00 & 14011.25 & $1.709^{*}$ & $-17.1340^{*}$ & $18.830 *$ \\
\hline MCO-6 & $08 / 21 / 98$ & $08 / 21 / 98$ & . & 13600 & 13300.00 & . & $2.230^{*}$ & . & . \\
\hline $\mathrm{MCO}-7$ & $08 / 21 / 98$ & $08 / 21 / 98$ & & 16800 & . 17200.00 & & $-2.353^{*}$ & & . \\
\hline MORTANDAD AT GS-1 & $08 / 28 / 98$ & . & $08 / 28 / 98$ & 4640 & . & 3501.25 & 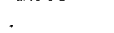 & $27.9748^{*}$ & \\
\hline MT-3 & . & $08 / 27 / 98$ & $09 / 04 / 98$ & . & 17500.00 & 18511.25 & . & 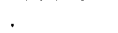 & $5.616^{*}$ \\
\hline MT-4 & . & $08 / 26 / 98$ & $05 / 14 / 98$ & . & 19200.00 & 17811.25 & . & . & $-7.504^{*}$ \\
\hline SACRED SPRING & . & $09 / 08 / 98$ & $09 / 08 / 98$ & . & 34.10 & -98.75 & . & . & $410.982 *$ \\
\hline TW-1 & . & $09 / 01 / 98$ & $05 / 28 / 98$ & . & 191.00 & 11.25 & . & . & $-177.750^{*}$ \\
\hline TW-3 & . & $09 / 01 / 98$ & $09 / 01 / 98$ & . & 3.14 & 441.25 & . & . & $197.174 *$ \\
\hline TW-4 & . & $09 / 01 / 98$ & $09 / 01 / 98$ & . & 2.07 & 231.25 & . & . & $196.451^{*}$ \\
\hline TW-8 & . & $09 / 02 / 98$ & $09 / 02 / 98$ & . & 18.50 & 311.25 & . & 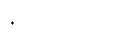 & $177.559^{*}$ \\
\hline
\end{tabular}


SUITE $=$ Rads Analyte $=$ PU-238 Unit $=\mathrm{PCL} /$

\begin{tabular}{|c|c|c|c|c|c|c|c|c|c|}
\hline Location & NM Date & EPA Date & $\begin{array}{l}\text { LANL } \\
\text { Date }\end{array}$ & $\begin{array}{l}\text { NMED } \\
\text { Result }\end{array}$ & $\begin{array}{l}\text { EPA } \\
\text { Result. }\end{array}$ & $\begin{array}{l}\text { LANL } \\
\text { Result }\end{array}$ & $\begin{array}{l}\text { NMED vs } \\
\text { EPA }\end{array}$ & $\begin{array}{l}\text { NMED vs } \\
\text { LANL }\end{array}$ & $\begin{array}{l}\text { LANL vs } \\
\text { EPA }\end{array}$ \\
\hline $\mathrm{MCO}-3$ & $08 / 20 / 98$ & . & $08 / 20 / 98$ & 0.700 & . & 0.8479 & 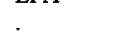 & $-19.111^{*}$ & . \\
\hline $\mathrm{MCO}-5$ & $08 / 20 / 98$ & . & $05 / 27 / 98$ & 0.006 & . & 0.0226 & . & $-116.121 *$ & . \\
\hline MORTANDAD AT GS-1 & $08 / 28 / 98$ & . & $08 / 28 / 98$ & 10.700 & . & 52.0198 & . & $-131.760^{*}$ & . \\
\hline TW-8 & $09 / 02 / 98$ & . & $09 / 02 / 98$ & 0.004 & . & -0.0292 & . & $-263.524^{*}$ & . \\
\hline
\end{tabular}

- SUITE $=$ Rads Analyte $=$ PU -239 Unit $=\mathrm{PCI} / \mathrm{L}$

\begin{tabular}{|c|c|c|c|c|c|c|c|c|c|}
\hline Location & NM Date & EPA Date & $\begin{array}{l}\text { LANL } \\
\text { Date }\end{array}$ & $\begin{array}{l}\text { NMED } \\
\text { Result }\end{array}$ & $\begin{array}{l}\text { EPA } \\
\text { Result }\end{array}$ & $\begin{array}{l}\text { LANL } \\
\text { Result }\end{array}$ & $\begin{array}{l}\text { NMED vs } \\
\text { EPA }\end{array}$ & $\begin{array}{l}\text { NMED vs } \\
\text { LANL }\end{array}$ & $\begin{array}{l}\text { LANL vs } \\
\text { EPA }\end{array}$ \\
\hline $\mathrm{MCO}-3$ & $08 / 20 / 98$ & . & $08 / 20 / 98$ & 0.145 & 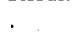 & 0.2054 & . & $-34.457 *$ & . \\
\hline MCO-5 & $08 / 20 / 98$ & . & $05 / 27 / 98$ & 0.034 & . & 0.0169 & . & $67.388^{*}$ & . \\
\hline MORTANDAD AT GS-1 & $08 / 28 / 98$ & . & $08 / 28 / 98$ & 2.960 & . & 16.9102 & . & $-140.413^{*}$ & : \\
\hline TW-8 & $09 / 02 / 98$ & . & $09 / 02 / 98$ & 0.004 & . & -0.0064 & . & $-856.410^{*}$ & . \\
\hline
\end{tabular}

SUTE $=$ Rads Analyte $=$ SR-90 Unit $=$ PCVI

\begin{tabular}{|c|c|c|c|c|c|c|c|c|c|}
\hline Location & NM Date & EPA Date & $\begin{array}{l}\text { LANL } \\
\text { Date }\end{array}$ & $\begin{array}{l}\text { NMED } \\
\text { Result }\end{array}$ & $\begin{array}{l}\text { EPA } \\
\text { Result }\end{array}$ & $\begin{array}{l}\text { LANL } \\
\text { Result }\end{array}$ & $\begin{array}{l}\text { NMED vs } \\
\text { EPA }\end{array}$ & $\begin{array}{l}\text { NMED vs } \\
\text { LANL }\end{array}$ & $\begin{array}{l}\text { LANL vs } \\
\text { EPA }\end{array}$ \\
\hline APCO-1 & . & $09 / 03 / 98$ & $09 / 03 / 98$ & . & 0.0600 & 0.0650 & . & . & $8.000^{*}$ \\
\hline LAO-2 & . & $08 / 31 / 98$ & $08 / 31 / 98$ & . & 7.2100 & 13.5750 & . & . & $61.246 *$ \\
\hline LAO-3A & . & $08 / 31 / 98$ & $08 / 31 / 98$ & . & 15.2000 & 33.8850 & . & . & $76.133^{*}$ \\
\hline $\mathrm{LAO}-4.5 \mathrm{C}$ & . & $08 / 31 / 98$ & $05 / 14 / 98$ & 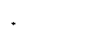 & 0.9100 & 2.0150 & & . & $75.556^{*}$ \\
\hline $\mathrm{MCO}-2$ & $08 / 19 / 98$ & $08 / 19 / 98$ & . & 0.79 & 0.4400 & . & $56.911^{*}$ & . & . \\
\hline $\mathrm{MCO}-3$ & $08 / 20 / 98$ & $08 / 20 / 98$ & $08 / 20 / 98$ & 47.20 & 28.1000 & 45.9250 & $50.730^{*}$ & $2.738^{*}$ & $48.159^{*}$ \\
\hline $\mathrm{MCO}-4 \mathrm{~B}$ & & $08 / 20 / 98$ & $05 / 27 / 98$ & 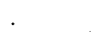 & 32.4000 & 48.3850 & & & $39.574 *$ \\
\hline $\mathrm{MCO}-5$ & $08 / 20 / 98$ & $08 / 20 / 98$ & $05 / 27 / 98$ & 35.10 & 19.0000 & 35.9550 & $59.519^{*}$ & $-2.407^{*}$ & $61.705^{*}$ \\
\hline MCO-6 & $08 / 21 / 98$ & $08 / 21 / 98$ & . & 31.70 & 63.5000 & . & $-66.807^{*}$ & . & . \\
\hline $\mathrm{MCO}-7$ & $08 / 21 / 98$ & $08 / 21 / 98$ & . & 0.89 & 0.5800 & . & $42.177^{*}$ & . & . \\
\hline MCWB-7.7B & $08 / 26 / 98$ & $08 / 26 / 98$ & $\therefore$ & $<0.50$ & 0.1200 & & 122.581 & & . \\
\hline MORTANDAD AT GS-1 & $08 / 28 / 98$ & . & $08 / 28 / 98$ & 12.00 & . & 10.9850 & . & $8.832 *$ & . \\
\hline MT-3 & $08 / 27 / 98$ & $08 / 27 / 98$ & $09 / 04 / 98$ & $<0.55$ & 0.0100 & 0.4650 & 192.857 & 16.749 & $191.579 *$ \\
\hline MT-4 & . & $08 / 26 / 98$ & $05 / 14 / 98$ & . & -0.0500 & 0.4390 & . & . & $251.414^{*}$ \\
\hline TA-50 OUTFALI & $08 / 28 / 98$ & $08 / 28 / 98$ & . & 14.70 & 0.3900 & & $189.662 *$ & . & . \\
\hline TW-1 & & $09 / 01 / 98$ & 05/28/98 & & 0.8100 & -0.0250 & & & $-212.739 *$ \\
\hline TW-2A & $09 / 01 / 98$ & $09 / 01 / 98$ & $.09 / 01 / 98$ & 1.20 & 0.0200 & 0.2950 & $193.443^{*}$ & $121.070^{*}$ & $174.603^{*}$ \\
\hline TW-3 & . & $09 / 01 / 98$ & $09 / 01 / 98$ & . & 0.2500 & 0.4650 & . & . & $60.140^{*}$ \\
\hline TW-8 & $09 / 02 / 98$ & $09 / 02 / 98$ & $09 / 02 / 98$ & $<0.58$ & -0.1500 & 0.6150 & 339.535 & -5.858 & $329.032 *$ \\
\hline
\end{tabular}

SUITE $=$ Rads Analyte $=$ U-234 Unit $=$ PCI $/ \mathrm{L}$

\begin{tabular}{|c|c|c|c|c|c|c|c|c|c|}
\hline Location & NM Date & EPA Date & $\begin{array}{l}\text { LANL } \\
\text { Date }\end{array}$ & $\begin{array}{l}\text { NMED } \\
\text { Result }\end{array}$ & $\begin{array}{l}\text { EPA } \\
\text { Result }\end{array}$ & $\begin{array}{l}\text { LANL } \\
\text { Result }\end{array}$ & $\begin{array}{l}\text { NMED vs } \\
\text { EPA }\end{array}$ & $\begin{array}{l}\text { NMED vs } \\
\text { LANL }\end{array}$ & $\begin{array}{l}\text { LANL vs } \\
\text { EPA }\end{array}$ \\
\hline $\mathrm{MCO}-2$ & $08 / 19 / 98$ & $08 / 19 / 98$ & . & 0.35 & 0.75000 & . & $-72.7273 *$ & . & er \\
\hline $\mathrm{MCO}-3$ & $08 / 20 / 98$ & $08 / 20 / 98$ & . & 5.67 & 5.62000 & . & $0.8857 *$ & . & . \\
\hline MCO-5 & $08 / 20 / 98$ & $08 / 20 / 98$ & . & 1.18 & 0.59000 & . & $66.6667^{*}$ & . & . \\
\hline MCO-6 & $08 / 21 / 98$ & $08 / 21 / 98$ & . & 1.46 & 3.23000 & ${ }^{\circ}$ & $-75.4797^{*}$ & . & . \\
\hline MCWB-7.7B & $08 / 26 / 98$ & $08 / 26 / 98$ & . & 0.90 & 0.65000 & . & $32.2581^{*}$ & . & . \\
\hline MT-3 & $08 / 27 / 98$ & $08 / 27 / 98$ & . & 0.83 & 0.60000 & . & $32,1678 *$ & $\therefore$ & . \\
\hline TA-50 OUTFALL & $08 / 28 / 98$ & $08 / 28 / 98$ & . & 1.03 & 0.95000 & . & $8.0808^{*}$ & . & . \\
\hline TW-1 & $09 / 01 / 98$ & $09 / 01 / 98$ & . & 1.70 & 2.31000 & . & $-30.4239 *$ & & . \\
\hline
\end{tabular}

Unfiltered

SUTTE $=$ Rads Analyte $=\mathrm{U}-235$ Unit $=\mathrm{PCI} / \mathrm{L}$

\begin{tabular}{|c|c|c|c|c|c|c|c|c|c|}
\hline Location & NM Date & EPA Date & $\begin{array}{l}\text { LANL } \\
\text { Date }\end{array}$ & $\begin{array}{l}\text { NMED } \\
\text { Result }\end{array}$ & $\begin{array}{l}\text { EPA } \\
\text { Result }\end{array}$ & $\begin{array}{l}\text { LANL } \\
\text { Result }\end{array}$ & $\begin{array}{l}\text { NMED vs } \\
\text { EPA }\end{array}$ & $\begin{array}{l}\text { NMED vs } \\
\text { LANL }\end{array}$ & $\begin{array}{l}\text { LANL vs } \\
\text { EPA }\end{array}$ \\
\hline $\mathrm{MCO}-2$ & $08 / 19 / 98$ & $08 / 19 / 98$ & . & 0.046 & 0.17000 & . & $-114.815^{*}$ & . & . \\
\hline $\mathrm{MCO}-3$ & $08 / 20 / 98$ & $08 / 20 / 98$ & . & 0.177 & 0.34000 & . & $-63.056^{*}$ & . & . \\
\hline MCO-5 & $08 / 20 / 98$ & $08 / 20 / 98$ & . & 0.030 & 0.15000 & . & $-133.333^{*}$ & . & . \\
\hline MCO-6 & $08 / 21 / 98$ & $08 / 21 / 98$ & . & 0.059 & 0.08000 & . & $-30.216^{*}$ & . & . \\
\hline MCWB-7.7B & $08 / 26 / 98$ & $08 / 26 / 98$ & . & 0.023 & 0.08000 & . & $-110.680^{*}$ & . & . \\
\hline MT-3 & $08 / 27 / 98$ & $08 / 27 / 98$ & . & 0.053 & 0.10000 & . & $-61.438^{*}$ & . & . \\
\hline TA-50 OUTFALL & $08 / 28 / 98$ & $08 / 28 / 98$ & . & 0.034 & $<0.72000$ & . & -181.963 & . & . \\
\hline TW-1 & $09 / 01 / 98$ & $09 / 01 / 98$ & . & 0.100 & 0.01000 & . & $163.636^{*}$ & . & . \\
\hline TW-8 & $09 / 02 / 98$ & $09 / 02 / 98$ & . & 0.020 & 0.07000 & . & $-111.111^{*}$ & . & . \\
\hline
\end{tabular}

SUTTE $=$ Rads Analyte $=\mathrm{U}-238$ Unit $=$ PCU

\begin{tabular}{|c|c|c|c|c|c|c|c|c|c|}
\hline Location & NM Date & EPA Date & $\begin{array}{l}\text { LANL } \\
\text { Date }\end{array}$ & $\begin{array}{l}\text { NMED } \\
\text { Result }\end{array}$ & $\begin{array}{l}\text { EPA } \\
\text { Result }\end{array}$ & $\begin{array}{l}\text { LANL } \\
\text { Result }\end{array}$ & $\begin{array}{l}\text { NMED vs } \\
\text { EPA }\end{array}$ & $\begin{array}{l}\text { NMED vs } \\
\text { LANL }\end{array}$ & $\begin{array}{l}\text { LANL vs } \\
\text { EPA }\end{array}$ \\
\hline $\mathrm{MCO}-2$ & $08 / 19 / 98$ & $08 / 19 / 98$ & . & 0.36 & 0.67000 & . & $-60.1942 *$ & . & . \\
\hline $\mathrm{MCO}-3$ & $08 / 20 / 98$ & $08 / 20 / 98$ & . & 2.250 & 2.07000 & . & $8.3333 *$ & . & . \\
\hline $\mathrm{MCO}-5$ & $08 / 20 / 98$ & $08 / 20 / 98$ & . & 0.480 & 0.37000 & . & $25.8824^{*}$ & . & . \\
\hline $\mathrm{MCO}-6$ & $08 / 21 / 98$ & $08 / 21 / 98$ & . & 0.400 & 0.93000 & . & $-79.6992 *$ & . & . \\
\hline MCWB-7.7B & $08 / 26 / 98$ & $08 / 26 / 98$ & . & 0.740 & 0.44000 & . & $50.8475^{*}$ & . & . \\
\hline MT-3 & $08 / 27 / 98$ & $08 / 27 / 98$ & . & 0.850 & 0.83000 & . & $2.3810^{*}$ & . & . \\
\hline TA-50 OUTFALL & $08 / 28 / 98$ & $08 / 28 / 98$ & . & 1.100 & 0.79000 & . & $32.8042 *$ & . & . \\
\hline TW-1 & $09 / 01 / 98$ & $09 / 01 / 98$ & . & 0.900 & 0.92000 & . & $-2.1978^{*}$ & . & . \\
\hline \multirow[t]{2}{*}{ TW-8 } & $09 / 02 / 98$ & $09 / 02 / 98$ & . & 0.175 & 0.47000 & . & $-91.4729 *$ & . & \\
\hline & & & & & 31 & & & & \\
\hline
\end{tabular}


SUITE $=$ Metals Analyte $=$ AG Unit $=\mu \mathrm{G} / \mathrm{L}$

\begin{tabular}{|c|c|c|c|c|c|c|c|c|c|}
\hline Location & NM Date & EPA Date & $\begin{array}{l}\text { LANL } \\
\text { Date }\end{array}$ & $\begin{array}{l}\text { NMED } \\
\text { Result }\end{array}$ & $\begin{array}{l}\text { EPA } \\
\text { Result }\end{array}$ & $\begin{array}{l}\text { LANL } \\
\text { Result }\end{array}$ & $\begin{array}{l}\text { NMED vs } \\
\text { EPA }\end{array}$ & $\begin{array}{l}\text { NMED vs } \\
\text { LANL }\end{array}$ & $\begin{array}{l}\text { LANL vs } \\
\text { EPA }\end{array}$ \\
\hline DP SPRING & $09 / 02 / 98$ & . & $09 / 02 / 98$ & $<0.5$ & . & $<10$ & . & -180.952 & . \\
\hline PUEBLO AT SR-502 & $09 / 03 / 98$ & . & $09 / 03 / 98$ & $<0.5$ & . & $<10$ & . & -180.952 & 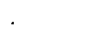 \\
\hline
\end{tabular}

SUITE $=$ Metals Analyte $=$ AL Unit $=\mu \mathrm{G} / \mathrm{L}$

\begin{tabular}{|c|c|c|c|c|c|c|c|c|c|}
\hline Location & NM Date & EPA Date & $\begin{array}{l}\text { LANL } \\
\text { Date }\end{array}$ & $\begin{array}{l}\text { NMED } \\
\text { Result }\end{array}$ & $\begin{array}{l}\text { EPA } \\
\text { Result }\end{array}$ & $\begin{array}{l}\text { LANL } \\
\text { Result }\end{array}$ & $\begin{array}{l}\text { NMED vs } \\
\text { EPA }\end{array}$ & $\begin{array}{l}\text { NMED vs } \\
\text { LANL }\end{array}$ & $\begin{array}{l}\text { LANL vs } \\
\text { EPA }\end{array}$ \\
\hline $\mathrm{APCO}-1$ & . & $09 / 03 / 98$ & $09 / 03 / 98$ & . & 33.800 & $<50$ & . & . & 38.663 \\
\hline BASALT SPRING & & $09 / 09 / 98$ & $06 / 04 / 98$ & 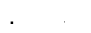 & 31.200 & $<50$ & . & . & 46.305 \\
\hline DP SPRING & $09 / 02 / 98$ & $09 / 02 / 98$ & $09 / 02 / 98$ & 1600 & 707.000 & 272 & $77.417^{*}$ & $141.880^{*}$ & $-88.866^{*}$ \\
\hline LAO-0.91 & $09 / 03 / 98$ & . & . & 500 & 612.000 & . & $-20.144^{*}$ & . & . \\
\hline LAO-1.2 & $08 / 31 / 98$ & $08 / 31 / 98$ & & 200 & 152.000 & 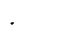 & $27.273^{*}$ & . & . \\
\hline LAO-2 & . & $08 / 31 / 98$ & $08 / 31 / 98$ & . & 577.000 & 495 & . & . & $-15.299^{*}$ \\
\hline LAO-3A & . & $08 / 31 / 98$ & $08 / 31 / 98$ & . & 195.000 & 250 & . & . & $24.719^{*}$ \\
\hline LAO-4.5C & . & $08 / 31 / 98$ & $05 / 14 / 98$ & . & 248.000 & 270 & . & . & $8.494^{*}$ \\
\hline $\mathrm{MCO}-3$ & . & $08 / 20 / 98$ & $08 / 20 / 98$ & . & 61.000 & 387 & . & . & $145.536^{*}$ \\
\hline $\mathrm{MCO}-4 \mathrm{~B}$ & . & $08 / 20 / 98$ & $05 / 27 / 98$ & . & 150.000 & $<50$ & . & . & -100.000 \\
\hline $\mathrm{MCO}-5$ & . & $08 / 20 / 98$ & $05 / 27 / 98$ & . & 121.000 & 61 & & . & $-65.934 *$ \\
\hline $\mathrm{MCO}-6$ & $08 / 21 / 98$ & $08 / 21 / 98$ & . & $<200$ & 96.800 & . & 69.542 & . & . \\
\hline $\mathrm{MCO}-7$ & $08 / 21 / 98$ & $08 / 21 / 98$ & & $<200$ & 365.000 & & -58.407 & . & . \\
\hline MT-3 & . & $08 / 27 / 98$ & $09 / 04 / 98$ & . & 87.800 & 66 & . & . & $-28.349^{*}$ \\
\hline MT-4 & . & $08 / 26 / 98$ & $05 / 14 / 98$ & . & 85.200 & 250 & . & . & $98.329^{*}$ \\
\hline OTOWI SPRING & . & $09 / 08 / 98$ & $09 / 08 / 98$ & . & 26.000 & $<50$ & . & . & 63.158 \\
\hline PUEBLO AT SR-502 & $09 / 03 / 98$ & . & $09 / 03 / 98$ & 200 & & $<50$ & . & 120.000 & . \\
\hline TA-50 OUTFALL & $08 / 28 / 98$ & $08 / 28 / 98$ & . & 200 & 312.000 & . & $-43.750^{*}$ & . & . \\
\hline TW-8 & $09 / 02 / 98$ & $09 / 02 / 98$ & . & $<200$ & $<23.600$ & . & 157.782 & . & . \\
\hline
\end{tabular}

SUITE $=$ Metals Analyte $=$ AS Unit $=\mu G / L$

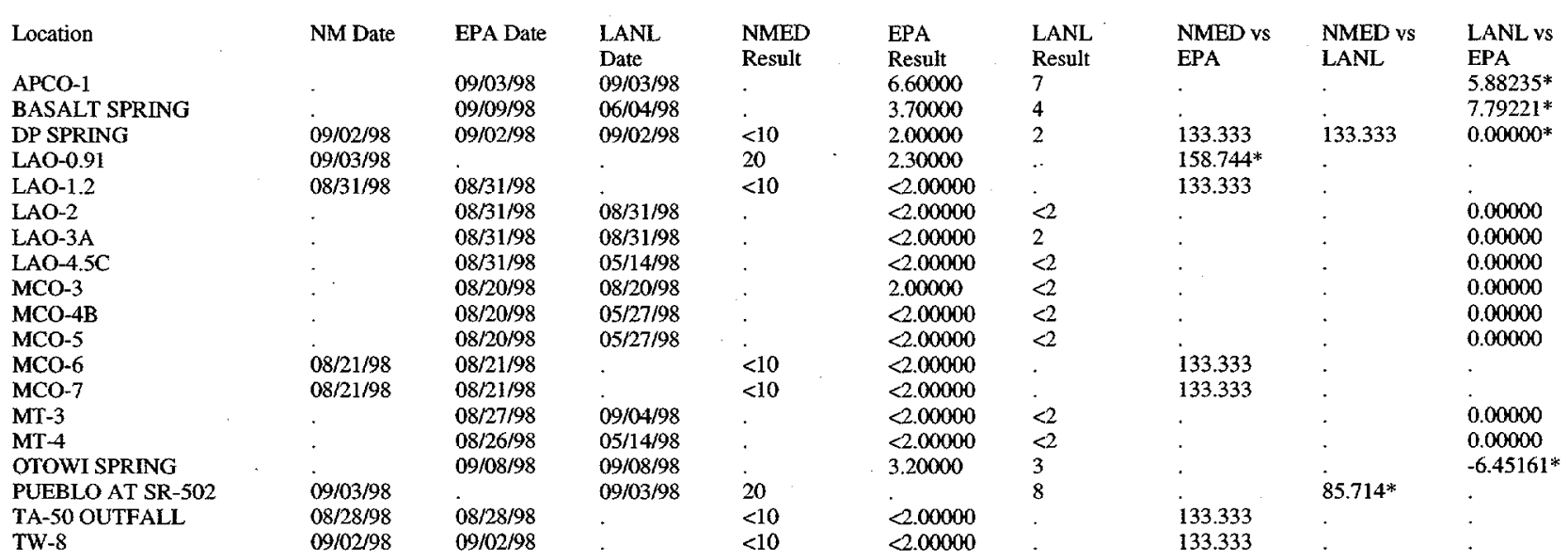

Filtered

SUITE $=$ Metals Analyte $=$ B Unit $=\mu G /$

\begin{tabular}{|c|c|c|c|c|c|c|c|c|c|}
\hline Location & NM Date & EPA Date & $\begin{array}{l}\text { LANL } \\
\text { Date }\end{array}$ & $\begin{array}{l}\text { NMED } \\
\text { Result }\end{array}$ & $\begin{array}{l}\text { EPA } \\
\text { Result }\end{array}$ & $\begin{array}{l}\text { LANL } \\
\text { Result }\end{array}$ & $\begin{array}{l}\text { NMED vs } \\
\text { EPA }\end{array}$ & $\begin{array}{l}\text { NMED vs } \\
\text { LANL }\end{array}$ & $\begin{array}{l}\text { LANL vs } \\
\text { EPA }\end{array}$ \\
\hline APCO-1 & . & $09 / 03 / 98$ & $09 / 03 / 98$ & . & 314.000 & 273 & . & . & $-13.969^{*}$ \\
\hline BASALT SPRING & & $09 / 09 / 98$ & $06 / 04 / 98$ & . & 262.000 & 228 & - & & $-13.878 *$ \\
\hline DP SPRING & $09 / 02 / 98$ & $09 / 02 / 98$ & $09 / 02 / 98$ & $<50$ & 62.900 & 43 & -22.852 & 15.0538 & $-37.583^{*}$ \\
\hline LAO-0.91 & $09 / 03 / 98$ & & . & $<50$ & 40.300 & $0^{\circ}$ & 21.484 & . & . \\
\hline LAO-1.2 & 08/31/98 & $08 / 31 / 98$ & & 190 & 31.900 & & $142.497^{*}$ & . & . \\
\hline LAO-2 & . & $08 / 31 / 98$ & $08 / 31 / 98$ & . & 103.000 & 59 & . & . & $-54.321^{*}$ \\
\hline LAO-3A & . & $08 / 31 / 98$ & $08 / 31 / 98$ & . & 57.000 & 60 & . & . & $5.128 *$ \\
\hline $\mathrm{LAO}-4.5 \mathrm{C}$ & . & $08 / 31 / 98$ & $05 / 14 / 98$ & . & 77.300 & $<20$ & . & . & -117.780 \\
\hline $\mathrm{MCO}-3$ & . & $08 / 20 / 98$ & $08 / 20 / 98$ & . & 220.000 & 170 & . & . & $-25.641^{*}$ \\
\hline $\mathrm{MCO}-4 \mathrm{~B}$ & . & $08 / 20 / 98$ & $05 / 27 / 98$ & . & 113.000 & 64 & . & . & $-55.367 *$ \\
\hline $\mathrm{MCO}-5$ & . & $08 / 20 / 98$ & $05 / 27 / 98$ & . & 114.000 & 77 & . & . & $-38.743^{*}$ \\
\hline $\mathrm{MCO}-6$ & $08 / 21 / 98$ & $08 / 21 / 98$ & . & $<100$ & 90.900 & . & 9.534 & . & . \\
\hline $\mathrm{MCO}-7$ & $08 / 21 / 98$ & $08 / 21 / 98$ & . & $<100$ & 107.000 & $\therefore$ & -6.763 & . & . \\
\hline MORTANDAD AT GS- 1 & $08 / 28 / 98$ & & $08 / 28 / 98$ & $<100$ & & 66 & . & 40.9639 & . \\
\hline MT-3 & . & $08 / 27 / 98$ & $09 / 04 / 98$ & . & 102.000 & 86 & . & . & $-17.021 *$ \\
\hline MT-4 & . & $08 / 26 / 98$ & $05 / 14 / 98$ & . & 125.000 & 81 & . & . & $-42.718^{*}$ \\
\hline OTOWI SPRING & . & $09 / 08 / 98$ & $09 / 08 / 98$ & - & 119.000 & 57 & . & . & $-70.455^{*}$ \\
\hline PUEBLO AT SR-502 & $09 / 03 / 98$ & . & $09 / 03 / 98$ & 360 & & 303 & . & $17.1946^{*}$ & . \\
\hline TA-50 OUTFALL & $08 / 28 / 98$ & $08 / 28 / 98$ & + & $<100$ & 106.000 & . & -5.825 & . & . \\
\hline TW-8 & $09 / 02 / 98$ & $09 / 02 / 98$ & . & $<100$ & 36.000 & . & 94.118 & . & . \\
\hline
\end{tabular}


SUITE $=$ Metals Analyte $=$ BA Unit $=\mu \mathrm{G} / \mathrm{L}$

\begin{tabular}{|c|c|c|c|c|c|c|c|c|c|}
\hline Location & NM Date & EPA Date & $\begin{array}{l}\text { LANL } \\
\text { Date }\end{array}$ & $\begin{array}{l}\text { NMED } \\
\text { Result }\end{array}$ & $\begin{array}{l}\text { EPA } \\
\text { Result }\end{array}$ & $\begin{array}{l}\text { LANL } \\
\text { Result }\end{array}$ & $\begin{array}{l}\text { NMED vs } \\
\text { EPA }\end{array}$ & $\begin{array}{l}\text { NMED vs } \\
\text { LANL }\end{array}$ & $\begin{array}{l}\text { LANL vs } \\
\text { EPA }\end{array}$ \\
\hline APCO-1 & . & $09 / 03 / 98$ & $09 / 03 / 98$ & . & 43.300 & 45 & . & . & $3.8505^{\star}$ \\
\hline BASALT SPRING & & $09 / 09 / 98$ & $06 / 04 / 98$ & . & 62.600 & 70 & 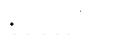 & 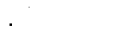 & $11.1614 *$ \\
\hline DP SPRING & $09 / 02 / 98$ & $09 / 02 / 98$ & $09 / 02 / 98$ & 51 & 40.800 & 41 & $22.222 *$ & $21.739 *$ & $0.4890^{*}$ \\
\hline LAO-0.91 & $09 / 03 / 98$ & & . & 48 & 36.000 & . & $28.571 *$ & . & . \\
\hline LAO-1.2 & $08 / 31 / 98$ & $08 / 31 / 98$ & . & 45 & 39.000 & . & $14.286^{*}$ & . & . \\
\hline LAO-2 & . & $08 / 31 / 98$ & $08 / 31 / 98$ & . & 52.600 & 53 &.$\quad$. & . & $0.7576^{*}$ \\
\hline LAO-3A & . & $08 / 31 / 98$ & $08 / 31 / 98$ & . & 47.100 & 49 & . & . & $3.9542 *$ \\
\hline $\mathrm{LAO}-4.5 \mathrm{C}$ & . & $08 / 31 / 98$ & $05 / 14 / 98$ & . & 40.200 & 35 & . & . & $-13.8298^{*}$ \\
\hline $\mathrm{MCO}-3$ & . & $08 / 20 / 98$ & $08 / 20 / 98$ & . & 62.400 & 65 & : & . & $4.0816^{*}$ \\
\hline $\mathrm{MCO}-4 \mathrm{~B}$ & . & $08 / 20 / 98$ & $05 / 27 / 98$ & . & 78.800 & 87 & . & $\therefore$ & $9.8914^{*}$ \\
\hline $\mathrm{MCO}-5$ & . & $08 / 20 / 98$ & $05 / 27 / 98$ & . & 91.600 & 87 & & . & $-5.1512^{*}$ \\
\hline MCO-6 & $08 / 21 / 98$ & $08 / 21 / 98$ & . & $<100$ & 88.400 & . & 12.314 & . & . \\
\hline $\mathrm{MCO}-7$ & 08/21/98 & $08 / 21 / 98$ & . & 200 & 187.000 & & $6.718^{*}$ & & . \\
\hline MORTANDAD AT GS-1 & $08 / 28 / 98$ & . & $08 / 28 / 98$ & $<100$ & . & 30 & . & 107.692 & : \\
\hline MT-3 & . & $08 / 27 / 98$ & $09 / 04 / 98$ & . & 146.000 & 148 & . & . & $1.3605^{*}$ \\
\hline MT-4 & . & $08 / 26 / 98$ & $05 / 14 / 98$ & . & 105.000 & 110 & . & . & $4.6512 *$ \\
\hline OTOWI SPRING & - & $09 / 08 / 98$ & $09 / 08 / 98$ & . & 144.000 & 144 & . & . & $0.0000^{*}$ \\
\hline PUEBLO AT SR-502 & $09 / 03 / 98$ & . & $09 / 03 / 98$ & 28 & . & 20 & . & $33.333^{*}$ & . \\
\hline TW-8 & $09 / 02 / 98$ & $09 / 02 / 98$ & . & $<100$ & 6.800 & . & 174.532 & . & . \\
\hline
\end{tabular}

SUTTE $=$ Metals Analyte $=$ BE Unit $=\mu \mathrm{G} / \mathrm{L}$

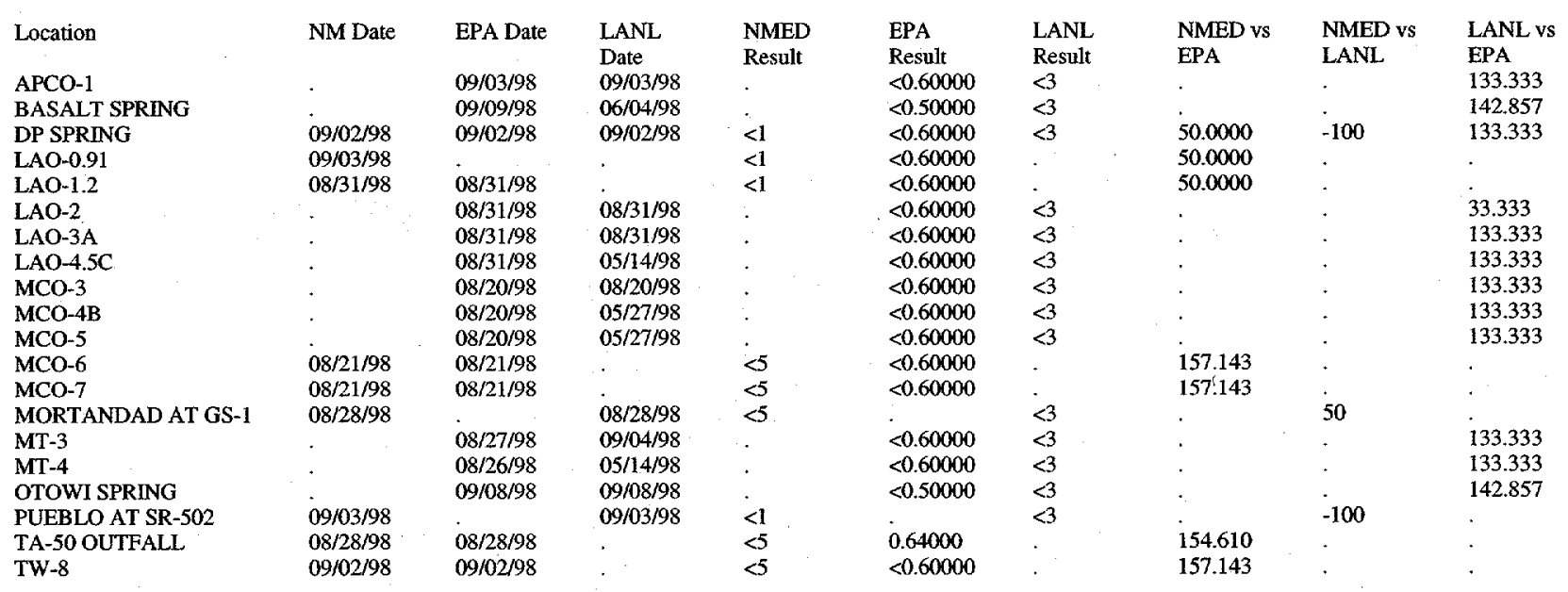

SUITE $=$ Metals Analyte $=\mathrm{CD}$ Unit $=\mu \mathrm{G} / \mathrm{L}$

\begin{tabular}{|c|c|c|c|c|c|c|c|c|c|}
\hline Location & NM Date & EPA Date & $\begin{array}{l}\text { LANL } \\
\text { Date }\end{array}$ & $\begin{array}{l}\text { NMED } \\
\text { Result }\end{array}$ & $\begin{array}{l}\text { EPA } \\
\text { Result }\end{array}$ & $\begin{array}{l}\text { LANL } \\
\text { Result }\end{array}$ & $\begin{array}{l}\text { NMED vs } \\
\text { EPA }\end{array}$ & $\begin{array}{l}\text { NMED vs } \\
\text { LANL }\end{array}$ & $\begin{array}{l}\text { LANL vs } \\
\text { EPA }\end{array}$ \\
\hline DP SPRING & $09 / 02 / 98$ & . & $09 / 02 / 98$ & $<1$ & & $<7$ & . & -150 & 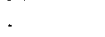 \\
\hline PUEBLO AT SR-502 & $09 / 03 / 98$ & . & $09 / 03 / 98$ & $<1$ & . & $<7$ & . & -150 & . \\
\hline
\end{tabular}

SUTTE $=$ Metals Analyte $=$ CO Unit $=\mu \mathrm{G} / \mathrm{L}$

\begin{tabular}{|c|c|c|c|c|c|c|c|c|c|}
\hline Location & NM Date & EPA Date & $\begin{array}{l}\text { LANL } \\
\text { Date }\end{array}$ & $\begin{array}{l}\text { NMED } \\
\text { Result }\end{array}$ & $\begin{array}{l}\text { EPA } \\
\text { Result }\end{array}$ & $\begin{array}{l}\text { LANL } \\
\text { Result }\end{array}$ & $\begin{array}{l}\text { NMED vs } \\
\text { EPA }\end{array}$ & $\begin{array}{l}\text { NMED vs } \\
\text { LANL }\end{array}$ & $\begin{array}{l}\text { LANL vs } \\
\text { EPA }\end{array}$ \\
\hline DP SPRING & $09 / 02 / 98$ & . & $09 / 02 / 98$ & $<1$ & 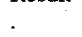 & $<8$ & & -155.556 & $\therefore$ \\
\hline MORTANDAD AT GS-1 & $08 / 28 / 98$ & . & $08 / 28 / 98$ & $<10$ & . & $<8$ & . & 22.222 & . \\
\hline PUEBLO AT SR-502 & $09 / 03 / 98$ & . & $09 / 03 / 98$ & 7 & . & $<8$ & . & -13.333 & . \\
\hline
\end{tabular}


SUTTE $=$ Metals Analyte $=$ CR Unit $=\mu \mathrm{G} /$

\begin{tabular}{|c|c|c|c|c|c|c|c|c|c|}
\hline Location & NM Date & EPA Date & $\begin{array}{l}\text { LANL } \\
\text { Date }\end{array}$ & $\begin{array}{l}\text { NMED } \\
\text { Result }\end{array}$ & $\begin{array}{l}\text { EPA } \\
\text { Result }\end{array}$ & $\begin{array}{l}\text { LANL } \\
\text { Result }\end{array}$ & $\begin{array}{l}\text { NMED vs } \\
\text { EPA }\end{array}$ & $\begin{array}{l}\text { NMED vs } \\
\text { LANL }\end{array}$ & $\begin{array}{l}\text { LANL vs } \\
\text { EPA }\end{array}$ \\
\hline $\mathrm{APCO}-1$ & . & $09 / 03 / 98$ & $09 / 03 / 98$ & . & $<4.2000$ & $<7$ & 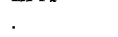 & 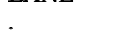 & 50.0000 \\
\hline BASALT SPRING & . & $09 / 09 / 98$ & $06 / 04 / 98$ & . & $<2.7000$ & $<7$ & . & . & 88.6598 \\
\hline DP SPRING & $09 / 02 / 98$ & $09 / 02 / 98$ & $09 / 02 / 98$ & $<50$ & $<4.2000$ & $<7$ & 169.004 & 150.877 & 50.0000 \\
\hline LAO-0.91 & $09 / 03 / 98$ & . & . & $<50$ & $<4.2000$ & . & 169.004 & $\therefore$ & . \\
\hline LAO-1.2 & $08 / 31 / 98$ & $08 / 31 / 98$ & . & $<50$ & 12.0000 & . & 122.581 & : & . \\
\hline $\mathrm{LAO}-2$ & 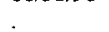 & $08 / 31 / 98$ & $08 / 31 / 98$ & . & $<4.2000$ & $<7$ & . & . & 50.0000 \\
\hline LAO-3A & . & $08 / 31 / 98$ & $08 / 31 / 98$ & . & $<4.2000$ & $<7$ & . & . & 50.0000 \\
\hline $\mathrm{LAO}-4.5 \mathrm{C}$ & . & $08 / 31 / 98$ & $05 / 14 / 98$ & . & $<4.2000$ & 8 & . & . & 62.2951 \\
\hline $\mathrm{MCO}-3$ & . & $08 / 20 / 98$ & $08 / 20 / 98$ & . & $<4.2000$ & $<7$ & . & . & 50.0000 \\
\hline MCO-4B & . & $08 / 20 / 98$ & $05 / 27 / 98$ & . & $<4.2000$ & $<7$ & . & . & 50.0000 \\
\hline $\mathrm{MCO}-5$ & & $08 / 20 / 98$ & $05 / 27 / 98$ & . & $<4.2000$ & $<7$ & . & . & 50.0000 \\
\hline $\mathrm{MCO}-6$ & $08 / 21 / 98$ & $08 / 21 / 98$ & . & $<10$ & $<4.2000$ & . & 81.690 & . & . \\
\hline $\mathrm{MCO}-7$ & $08 / 21 / 98$ & $08 / 21 / 98$ & & $<10$ & $<4.2000$ & . & 81.690 & $\cdot$ & . \\
\hline MORTANDAD AT GS- 1 & $08 / 28 / 98$ & . & $08 / 28 / 98$ & $<10$ & . & $<7$ & . & 35.294 & . \\
\hline MT-3 & . & $08 / 27 / 98$ & $09 / 04 / 98$ & . & $<4.2000$ & $<7$ & . & . & 50.0000 \\
\hline $\mathrm{MT}-4$ & . & $08 / 26 / 98$ & $05 / 14 / 98$ & . & 4.5000 & $<7$ & . & . & 43.4783 \\
\hline OTOWI SPRING & . & $09 / 08 / 98$ & $09 / 08 / 98$ & . & $<2.7000$ & $<7$ & . & & 88.6598 \\
\hline PUEBLO AT SR-502 & $09 / 03 / 98$ & . & $09 / 03 / 98$ & $<50$ & & $<7$ & . & 150.877 & . \\
\hline TA-50 OUTFALL & $08 / 28 / 98$ & $08 / 28 / 98$ & . & $<10$ & 5.2000 & . & 63.158 & . & . \\
\hline TW-8 & $09 / 02 / 98$ & $09 / 02 / 98$ & . & $<10$ & $<4.20000$ & . & 81.6901 & . & . \\
\hline
\end{tabular}

SUITE $=$ Metals Analyte $=\mathrm{CU}$ Unit $=\mu \mathrm{G} / \mathrm{L}$

\begin{tabular}{|c|c|c|c|c|c|c|c|c|c|}
\hline Location & NM Date & EPA Date & $\begin{array}{l}\text { LANL } \\
\text { Date }\end{array}$ & $\begin{array}{l}\text { NMED } \\
\text { Result }\end{array}$ & $\begin{array}{l}\text { EPA } \\
\text { Result }\end{array}$ & $\begin{array}{l}\text { LANL } \\
\text { Result }\end{array}$ & $\begin{array}{l}\text { NMED vs } \\
\text { EPA }\end{array}$ & $\begin{array}{l}\text { NMED vs } \\
\text { LANL }\end{array}$ & $\begin{array}{l}\text { LANL vs } \\
\text { EPA }\end{array}$ \\
\hline APCO-1 & . & $09 / 03 / 98$ & $09 / 03 / 98$ & . & $<6.200$ & $<10$ & . & . & 46.914 \\
\hline BASALT SPRING & & $09 / 09 / 98$ & $06 / 04 / 98$ & . & 14.800 & $<10$ & & & -38.710 \\
\hline DP SPRING & $09 / 02 / 98$ & $09 / 02 / 98$ & $09 / 02 / 98$ & $<20$ & $<6.200$ & $<10$ & 105.344 & 66.6667 & 46.914 \\
\hline LAO-0.91 & $09 / 03 / 98$ & & . & $<20$ & $<6.200$ & . & 105.344 & . & . \\
\hline $\mathrm{LAO}-1.2$ & $08 / 31 / 98$ & $08 / 31 / 98$ & . & $<20$ & $<6.200$ & . & 105.344 & . & . \\
\hline $\mathrm{LAO}-2$ & . & $08 / 31 / 98$ & $08 / 31 / 98$ & . & $<6.200$ & $<10$ & . & . & 46.914 \\
\hline $\mathrm{LAO}-3 \mathrm{~A}$ & . & $08 / 31 / 98$ & $08 / 31 / 98$ & . & $<6.200$ & $<10$ & . & . & 46.914 \\
\hline $\mathrm{LAO}_{-4.5 \mathrm{C}}$ & . & $08 / 31 / 98$ & $05 / 14 / 98$ & . & $<6.200$ & $<10$ & . & . & 46.914 \\
\hline $\mathrm{MCO}-3$ & . & $08 / 20 / 98$ & $08 / 20 / 98$ & . & 17.000 & 15 & . & . & $-12.500^{*}$ \\
\hline $\mathrm{MCO}-4 \mathrm{~B}$ & . & $08 / 20 / 98$ & $05 / 27 / 98$ & . & $<6.200$ & $<10$ & . & . & 46.914 \\
\hline MCO-5 & & $08 / 20 / 98$ & $05 / 27 / 98$ & . & 9.000 & $<10$ & . & . & 10.526 \\
\hline MCO-6 & $08 / 21 / 98$ & $08 / 21 / 98$ & . & $<10$ & $<6.200$ & . & 46.914 & . & . \\
\hline MCO-7 & $08 / 21 / 98$ & $08 / 21 / 98$ & . & $<10$ & $<6.200$ & . & 46.914 & 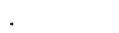 & . \\
\hline MORTANDAD AT GS-1 & $08 / 28 / 98$ & . & $08 / 28 / 98$ & 100 & . & 101 & . & $-0.9950^{*}$ & \\
\hline $\mathrm{MT}-3$ & 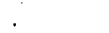 & $08 / 27 / 98$ & $09 / 04 / 98$ & . & $<6.200$ & $<10$ & . & . & 46.914 \\
\hline MT-4 & . & $08 / 26 / 98$ & $05 / 14 / 98$ & . & 8.200 & $<10$ & . & . & 19.780 \\
\hline OTOWI SPRING & & $09 / 08 / 98$ & $09 / 08 / 98$ & . & $<2.900$ & $<10$ & . & . & 110.078 \\
\hline PUEBLO AT SR-502 & $09 / 03 / 98$ & . & $09 / 03 / 98$ & $<20$ & & $<10$ & . & 66.6667 & . \\
\hline TW-8 & $09 / 02 / 98$ & $09 / 02 / 98$ & . & $<10$ & $<6.200$ & . & 46.914 & . & \\
\hline
\end{tabular}

SUITE $=$ Metals Analyte $=$ FE Unit $=\mu G / L$

\begin{tabular}{|c|c|c|c|c|c|c|c|c|c|}
\hline Location & NM Date & EPA Date & $\begin{array}{l}\text { LANL } \\
\text { Date }\end{array}$ & $\begin{array}{l}\text { NMED } \\
\text { Result }\end{array}$ & $\begin{array}{l}\text { EPA } \\
\text { Result }\end{array}$ & $\begin{array}{l}\text { LANL } \\
\text { Result }\end{array}$ & $\begin{array}{l}\text { NMED vs } \\
\text { EPA }\end{array}$ & $\begin{array}{l}\text { NMED vs } \\
\text { LANL }\end{array}$ & $\begin{array}{l}\text { LANL vs } \\
\text { EPA }\end{array}$ \\
\hline APCO-1 & . & $09 / 03 / 98$ & $09 / 03 / 98$ & . & 452.000 & 455 & . & 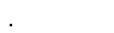 & $0.662^{*}$ \\
\hline BASALT SPRING & & $09 / 09 / 98$ & $06 / 04 / 98$ & . & 38.100 & 130 & & & $109.340 *$ \\
\hline DP SPRING & $09 / 02 / 98$ & $09 / 02 / 98$ & $09 / 02 / 98$ & 600 & 376.000 & 177 & $45.902 *$ & $108.880^{*}$ & $-71.971 *$ \\
\hline $\mathrm{LAO}-0.91$ & $09 / 03 / 98$ & & . & 100 & 329.000 & 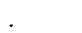 & $-106.760^{*}$ & . & . \\
\hline LAO-1.2 & $08 / 31 / 98$ & $08 / 31 / 98$ & . & $<100$ & 72.900 & 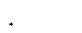 & 31.348 & . & . \\
\hline $\mathrm{LAO}-2$ & . & $08 / 31 / 98$ & $08 / 31 / 98$ & . & 5.000 & 250 & . & . & $192.157 *$ \\
\hline LAO-3A & . & 08/31/98 & $08 / 31 / 98$ & . & 111.000 & 112 & . & . & $0.897 *$ \\
\hline $\mathrm{LAO}-4.5 \mathrm{C}$ & . & $08 / 31 / 98$ & $05 / 14 / 98$ & . & 139.000 & 54 & . & . & $-88.083^{*}$ \\
\hline $\mathrm{MCO}-3$ & . & $08 / 20 / 98$ & $08 / 20 / 98$ & . & 34.700 & $<40$ & . & . & 14.190 \\
\hline MCO-4B & . & $08 / 20 / 98$ & $05 / 27 / 98$ & . & 71.500 & $<40$ & . & . & -56.502 \\
\hline $\mathrm{MCO}-5$ & & $08 / 20 / 98$ & $05 / 27 / 98$ & . & 56.600 & $<40$ & . & . & -34.369 \\
\hline MCO-6 & $08 / 21 / 98$ & $08 / 21 / 98$ & . & $<100$ & 63.300 & . & 44.948 & . & . \\
\hline $\mathrm{MCO}-7$ & $08 / 21 / 98$ & $08 / 21 / 98$ & . & $<100$ & 213.000 & . & -72.204 & . & . \\
\hline MORTANDAD AT GS-1 & $08 / 28 / 98$ & & $08 / 28 / 98$ & $<100$ & & $<40$ & . & 85.714 & \\
\hline MT-3 & . & $08 / 27 / 98$ & $09 / 04 / 98$ & . & 60.300 & 250 & . & 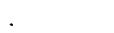 & $122.269 *$ \\
\hline MT-4 & . & $08 / 26 / 98$ & $05 / 14 / 98$ & . & 277.000 & 74 & . & . & $-115.670^{*}$ \\
\hline OTOWI SPRING & & $09 / 08 / 98$ & $09 / 08 / 98$ & 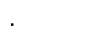 & 33.800 & $<40$ & . & . & 16.802 \\
\hline PUEBLO AT SR-502 & $09 / 03 / 98$ & . & $09 / 03 / 98$ & 800 & . & 593 & . & $29.720^{*}$ & . \\
\hline TW-8 & $09 / 02 / 98$ & $09 / 02 / 98$ & . & $<100$ & 57.800 & . & 53.485 & . & . \\
\hline
\end{tabular}


SUITE $=$ Metals Analyte $=$ MN Unit $=\mu G / L$

\begin{tabular}{|c|c|c|c|c|c|c|c|c|c|}
\hline Location & NM Date & EPA Date & $\begin{array}{l}\text { LANL } \\
\text { Date }\end{array}$ & $\begin{array}{l}\text { NMED } \\
\text { Result }\end{array}$ & $\begin{array}{l}\text { EPA } \\
\text { Result }\end{array}$ & $\begin{array}{l}\text { LANL } \\
\text { Result }\end{array}$ & $\begin{array}{l}\text { NMED vs } \\
\text { EPA }\end{array}$ & $\begin{array}{l}\text { NMED vs } \\
\text { LANL }\end{array}$ & $\begin{array}{l}\text { LANL vs } \\
\text { EPA }\end{array}$ \\
\hline APCO-1 & . & $09 / 03 / 98$ & $09 / 03 / 98$ & . & 942.000 & 967 & 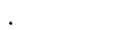 & . & $2.619^{*}$ \\
\hline BASALT SPRING & . & $09 / 09 / 98$ & $06 / 04 / 98$ & . & 0.910 & 4 & - & & $125866^{*}$ \\
\hline DP SPRING & $09 / 02 / 98$ & $09 / 02 / 98$ & $09 / 02 / 98$ & 4 & 8.300 & 5 & $-69.919^{*}$ & $-22.2222 *$ & $-49.624^{*}$ \\
\hline LAO-0.91 & $09 / 03 / 98$ & . & . & $<3$ & 2.400 & . & 22.222 & . & . \\
\hline LAO- 1.2 & $08 / 31 / 98$ & $08 / 31 / 98$ & . & $<3$ & 0.940 & . & 104.569 & . & - \\
\hline LAO-2 & . & $08 / 31 / 98$ & $08 / 31 / 98$ & . & 149.000 & $<2$ & . & . & -194.702 \\
\hline LAO-3A & . & $08 / 31 / 98$ & $08 / 31 / 98$ & . & 2.000 & $<3$ & . & . & 40.000 \\
\hline LAO-4.5C & . & $08 / 31 / 98$ & $05 / 14 / 98$ & . & 13.700 & $<2$ & . & . & -149.045 \\
\hline $\mathrm{MCO}-3$ & . & $08 / 20 / 98$ & $08 / 20 / 98$ & . & 3.900 & $<2$ &. & . & -64.407 \\
\hline MCO-4B & . & $08 / 20 / 98$ & $05 / 27 / 98$ & . & 2.500 & $<2$ & . & . & -22.222 \\
\hline MCO-5 & . & $08 / 20 / 98$ & $05 / 27 / 98$ & . & 2.900 & $<2$ & . & . & -36.735 \\
\hline MCO-6 & $08 / 21 / 98$ & $08 / 21 / 98$ & . & $<10$ & 2.400 & . & 122.581 & . & . \\
\hline $\mathrm{MCO}-7$ & $08 / 21 / 98$ & $08 / 21 / 98$ & & $<10$ & 2.900 & & 110.078 & & \\
\hline MORTANDAD AT GS-1 & $08 / 28 / 98$ & & $08 / 28 / 98$ & $<10$ & . & 8 & $\cdot$ & 22.2222 & . \\
\hline MT-3 & . & $08 / 27 / 98$ & $09 / 04 / 98$ & . & 1.000 & 12 & . & . & $169.231 *$ \\
\hline MT-4 & . & $08 / 26 / 98$ & $05 / 14 / 98$ & . & 3.000 & 3 & . & . & $0.000^{*}$ \\
\hline OTOWI SPRING & & $09 / 08 / 98$ & $09 / 08 / 98$ & & 1.700 & $<2$ & $\ddots$ & & 16.216 \\
\hline PUEBLO AT SR-502 & $09 / 03 / 98$ & & $09 / 03 / 98$ & 330 & $\cdot$ & 299 & & $9.8569^{*}$ & . \\
\hline TA-50 OUTFALL & $08 / 28 / 98$ & $08 / 28198$ & . & $<10$ & 6.400 & - & 43.902 & . & . \\
\hline TW-8- & $09 / 02 / 98$ & $09 / 02 / 98$ & . & $<10$ & 3.700 & . & 91.971 & . & + \\
\hline
\end{tabular}

SUITE $=$ Metals Analyte $=$ MO Unit $=\mu G /$

\begin{tabular}{|c|c|c|c|c|c|c|c|c|c|}
\hline Location & NM Date & EPA Date & $\begin{array}{l}\text { LANL } \\
\text { Date }\end{array}$ & $\begin{array}{l}\text { NMED } \\
\text { Result }\end{array}$ & $\begin{array}{l}\text { EPA } \\
\text { Result }\end{array}$ & $\begin{array}{l}\text { LANL } \\
\text { Result }\end{array}$ & $\begin{array}{l}\text { NMED vs } \\
\text { EPA }\end{array}$ & $\begin{array}{l}\text { NMED vs } \\
\text { LANL }\end{array}$ & $\begin{array}{l}\text { LANL vs } \\
\text { EPA }\end{array}$ \\
\hline APCO-1 & . & $09 / 03 / 98$ & $09 / 03 / 98$ & . & $<7.300$ & $<30$ & . & . & 121.716 \\
\hline BASALT SPRING & & $09 / 09 / 98$ & $06 / 04 / 98$ & . & $<8.400$ & $<30$ & + & . & 112.500 \\
\hline DP SPRING & $09 / 02 / 98$ & $09 / 02 / 98$ & $09 / 02 / 98$ & $<10$ & $<7.300$ & $<30$ & 31.2139 & -100 & 121.716 \\
\hline $\mathrm{LAO}-0.91$ & $09 / 03 / 98$ & & . & $<10$ & 9.200 & . & 8.3333 & . & . \\
\hline LAO- 1.2 & $08 / 31 / 98$ & $08 / 31 / 98$ & 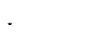 & $<10$ & 8.500 & . & 16.2162 & . & - \\
\hline $\mathrm{LAO}-2$ & . & $08 / 31 / 98$ & $08 / 31 / 98$ & 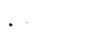 & 149.000 & 153 & & . & $2.649^{*}$ \\
\hline $\mathrm{LAO}-3 \mathrm{~A}$ & . & $08 / 31 / 98$ & $08 / 31 / 98$ & . & 317.000 & 328 &. & . & $3.411 *$ \\
\hline $\mathrm{LAO}-4.5 \mathrm{C}$ & . & $08 / 31 / 98$ & $05 / 14 / 98$ & . & 16.200 & $<30$ & . & . & 59.740 \\
\hline $\mathrm{MCO}-3$ & . & $08 / 20 / 98$ & $08 / 20 / 98$ & . & 156.000 & 157 & . & . & $0.639 *$ \\
\hline $\mathrm{MCO}-4 \mathrm{~B}$ & . & $08 / 20 / 98$ & $05 / 27 / 98$ & . & 127.000 & 122 & . & . & $-4.016^{*}$ \\
\hline MCO-5 & . & $08 / 20 / 98$ & $05 / 27 / 98$ & . & 110.000 & 129 & . & . & $15.900^{*}$ \\
\hline MCO-6 & $08 / 21 / 98$ & $08 / 21 / 98$ & . & 120 & 126.000 & . & $-4.8780^{*}$ & . & . \\
\hline $\mathrm{MCO}-7$ & $08 / 21 / 98$ & $08 / 21 / 98$ & . & 100 & 117.000 & . & $-15.6682 *$ & . & . \\
\hline MT-3 & . & $08 / 27 / 98$ & $09 / 04 / 98$ & . & 58.500 & 68 & . & . & $15.020 *$ \\
\hline MT-4 & . & $08 / 26 / 98$ & $05 / 14 / 98$ & . & 33.900 & $<30$ & . & . & -12.207 \\
\hline OTOWI SPRING & & $09 / 08 / 98$ & $09 / 08 / 98$ & & $<8.400$ & $<30$ & . & . & 112.500 \\
\hline PUEBLO AT SR-502 & $09 / 03 / 98$ & & $09 / 03 / 98$ & $<10$ & & $<30$ & + & -100 & . \\
\hline TA-50 OUTFALL & $08 / 28 / 98$ & $08 / 28 / 98$ & . & 80 & 118.000 & . & $-38.3838 *$ & . & . \\
\hline TW-8 & $09 / 02 / 98$ & $09 / 02 / 98$ & . & $<10$ & $<7.300$ & . & 31.2139 & & \\
\hline
\end{tabular}

SUITE $=$ Metals Analyte $=$ NI Unit $=\mu \mathrm{G} / \mathrm{L}$

\begin{tabular}{|c|c|c|c|c|c|c|c|c|c|}
\hline Location & NM Date & EPA Date & $\begin{array}{l}\text { LANL } \\
\text { Date }\end{array}$ & $\begin{array}{l}\text { NMED } \\
\text { Result }\end{array}$ & $\begin{array}{l}\text { EPA } \\
\text { Result }\end{array}$ & $\begin{array}{l}\text { LANL } \\
\text { Result }\end{array}$ & $\begin{array}{l}\text { NMED vs } \\
\text { EPA }\end{array}$ & $\begin{array}{l}\text { NMED vs } \\
\text { LANL }\end{array}$ & $\begin{array}{l}\text { LANL vs } \\
\text { EPA }\end{array}$ \\
\hline DP SPRING & 09/02/98 & . & $09 / 02 / 98$ & $<5$ & . & $<20$ & . & -120.000 & . \\
\hline PUEBLO AT SR-502 & $09 / 03 / 98$ & . & $09 / 03 / 98$ & 10 & & 20 & . & $-66.667^{*}$ & . \\
\hline
\end{tabular}

- SUTTE $=$ Metals Analyte $=P B$ Unit $=\mu G / \mathrm{L}$

\begin{tabular}{|c|c|c|c|c|c|c|c|c|c|}
\hline Location & NM Date & EPA Date & $\begin{array}{l}\text { LANL } \\
\text { Date }\end{array}$ & $\begin{array}{l}\text { NMED } \\
\text { Result }\end{array}$ & $\begin{array}{l}\text { EPA } \\
\text { Result }\end{array}$ & $\begin{array}{l}\text { LANL } \\
\text { Result }\end{array}$ & $\begin{array}{l}\text { NMED vs } \\
\text { EPA }\end{array}$ & $\begin{array}{l}\text { NMED vs } \\
\text { LANL }\end{array}$ & $\begin{array}{l}\text { LANL vs } \\
\text { EPA }\end{array}$ \\
\hline APCO-1 & . & 09/03/98 & $09 / 03 / 98$ & 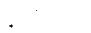 & 7.1000 & $<3$ & . & . & -81.188 \\
\hline BASALT SPRING & . & $09 / 09 / 98$ & $06 / 04 / 98$ & . & 5.1000 & $<3$ & . & . & -51.852 \\
\hline DP SPRING & $09 / 02 / 98$ & $09 / 02 / 98$ & $09 / 02 / 98$ & $<5$ & 7.2000 & $<3$ & -36.066 & 50.0000 & -82.353 \\
\hline LAO-0.91 & $09 / 03 / 98$ & . & . & $<5$ & 5.9000 & . & -16.514 & . & . \\
\hline LAO-1.2 & $08 / 31 / 98$ & $08 / 31 / 98$ & . & 5 & 4.0000 & . & $22.222 *$ & . & . \\
\hline LAO-2 & . & $08 / 31 / 98$ & $08 / 31 / 98$ & . & 2.6000 & $<3$ & . & . & 14.286 \\
\hline LAO-3A & . & $08 / 31 / 98$ & $08 / 31 / 98$ & . & 3.2000 & 61 & . & . & $180.0620 *$ \\
\hline $\mathrm{LAO}-4.5 \mathrm{C}$ & . & $08 / 31 / 98$ & $05 / 14 / 98$ & . & 5.7000 & $<3$ & . & . & -62.069 \\
\hline $\mathrm{MCO}-4 \mathrm{~B}$ & . & $08 / 20 / 98$ & $05 / 27 / 98$ & . & $<1.6000$ & $<3$ & . & . & 60.870 \\
\hline $\mathrm{MCO}-5$ & . & $08 / 20 / 98$ & $05 / 27 / 98$ & . & $<1.6000$ & $<3$ & . & . & 60.870 \\
\hline MCO-6 & $08 / 21 / 98$ & $08 / 21 / 98$ & . & $<3$ & 43.3000 & . & -174.082 & . & . \\
\hline $\mathrm{MCO}-7$ & $08 / 21 / 98$ & $08 / 21 / 98$ & . & $<3$ & 11.1000 & . & -114.894 & . & . \\
\hline MORTANDAD AT GS- 1 & $08 / 28 / 98$ & & $08 / 28 / 98$ & $<3$ & & 4 & . & -28.5714 & 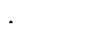 \\
\hline MT-3 & . & $08 / 27 / 98$ & $09 / 04 / 98$ & . & 15.2000 & $<3$ & . & . & -134.066 \\
\hline MT-4 & . & $08 / 26 / 98$ & $05 / 14 / 98$ & . & 10.5000 & $<3$ & . & . & -111.111 \\
\hline OTOWI SPRING & & $09 / 08 / 98$ & $09 / 08 / 98$ & . & 4.5000 & $<3$ & . & 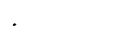 & -40.000 \\
\hline PUEBLO AT SR-502 & $09 / 03 / 98$ & . & $09 / 03 / 98$ & $<5$ & . & 3 & . & 50.0000 & . \\
\hline TW-8 & $09 / 02 / 98$ & $09 / 02 / 98$ & . & $<3$ & 4.8000 & . & -46.154 & . & . \\
\hline
\end{tabular}


SUTTE $=$ Metals Analyte $=$ SB Unit $=\mu G / L$

\begin{tabular}{|c|c|c|c|c|c|c|c|c|c|}
\hline Location & NM Date & EPA Date & $\begin{array}{l}\text { LANL } \\
\text { Date }\end{array}$ & $\begin{array}{l}\text { NMED } \\
\text { Result }\end{array}$ & $\begin{array}{l}\text { EPA } \\
\text { Result }\end{array}$ & $\begin{array}{l}\text { LANL } \\
\text { Result }\end{array}$ & $\begin{array}{l}\text { NMED vs } \\
\text { EPA }\end{array}$ & $\begin{array}{l}\text { NMED vs } \\
\text { LANL }\end{array}$ & $\begin{array}{l}\text { LANL vs } \\
\text { EPA }\end{array}$ \\
\hline DP SPRING & $09 / 02 / 98$ & . & $09 / 02 / 98$ & $<10$ & . & $<3$ & . & 107.692 & . \\
\hline PUEBLO AT SR-502 & $09 / 03 / 98$ & . & $09 / 03 / 98$ & $<10$ & . & $<3$ & . & 107.692 & . \\
\hline
\end{tabular}

SUITE $=$ Metals Analyte $=\mathrm{SN}$ Unit $=\mu \mathrm{G} / \mathrm{L}$

\begin{tabular}{|c|c|c|c|c|c|c|c|c|c|}
\hline Location & NM Date & EPA Date & $\begin{array}{l}\text { LANL } \\
\text { Date }\end{array}$ & $\begin{array}{l}\text { NMED } \\
\text { Result }\end{array}$ & $\begin{array}{l}\text { EPA } \\
\text { Result }\end{array}$ & $\begin{array}{l}\text { LANL } \\
\text { Result }\end{array}$ & $\begin{array}{l}\text { NMED vs } \\
\text { EPA }\end{array}$ & $\begin{array}{l}\text { NMED vs } \\
\text { LANL }\end{array}$ & $\begin{array}{l}\text { LANL vs } \\
\text { EPA }\end{array}$ \\
\hline DP SPRING & $09 / 02 / 98$ & . & $09 / 02 / 98$ & $<300$ & . & $<30$ & . & 163.636 & . \\
\hline PUEBLO AT SR-502 & $09 / 03 / 98$ & . & $09 / 03 / 98$ & $<300$ & & $<163$ & . & 59.179 & . \\
\hline
\end{tabular}

Filtered

- SUITE $=$ Metals Analyte $=$ SR Unit $=\mu G / L$

\begin{tabular}{|c|c|c|c|c|c|c|c|c|c|}
\hline Location & NM Date & EPA Date & $\begin{array}{l}\text { LANL } \\
\text { Date }\end{array}$ & $\begin{array}{l}\text { NMED } \\
\text { Result }\end{array}$ & $\begin{array}{l}\text { EPA } \\
\text { Result }\end{array}$ & $\begin{array}{l}\text { LANL } \\
\text { Result }\end{array}$ & $\begin{array}{l}\text { NMED vs } \\
\text { EPA }\end{array}$ & $\begin{array}{l}\text { NMED vs } \\
\text { LANL }\end{array}$ & $\begin{array}{l}\text { LANL vs } \\
\text { EPA }\end{array}$ \\
\hline DP SPRING & 09/02/98 & . & $09 / 02 / 98$ & 100 & . & 97 & . & $3.04569 *$ & . \\
\hline MORTANDAD AT GS- 1 & $08 / 28 / 98$ & . & $08 / 28 / 98$ & 70 & . & 75 & . & $-6.89655 *$ & . \\
\hline PUEBLO AT SR-502 & $09 / 03 / 98$ & . & $09 / 03 / 98$ & 90 & . & 87 & : & $3.38983 *$ & . \\
\hline
\end{tabular}

SUTTE $=$ Metals Analyte $=\mathrm{V}$ Unit $=\mu \mathrm{G} \Omega$

\begin{tabular}{|c|c|c|c|c|c|c|c|c|c|}
\hline Location & NM Date & EPA Date & $\begin{array}{l}\text { LANL } \\
\text { Date }\end{array}$ & $\begin{array}{l}\text { NMED } \\
\text { Result }\end{array}$ & $\begin{array}{l}\text { EPA } \\
\text { Result }\end{array}$ & $\begin{array}{l}\text { LANL } \\
\text { Result }\end{array}$ & $\begin{array}{l}\text { NMED vs } \\
\text { EPA }\end{array}$ & $\begin{array}{l}\text { NMED vs } \\
\text { LANL }\end{array}$ & $\begin{array}{l}\text { LANL vs } \\
\text { EPA }\end{array}$ \\
\hline DP SPRING & $09 / 02 / 98$ & . & $09 / 02 / 98$ & $<10$ & . & $<8$ & 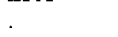 & 22.2222 & 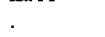 \\
\hline MORTANDAD AT GS-I & $08 / 28 / 98$ & . & $08 / 28 / 98$ & $<10$ & . & $<8$ & . & 22.2222 & . \\
\hline PUEBLO AT SR-502 & $09 / 03 / 98$ & . & $09 / 03 / 98$ & 10 & . & $<8$ & . & 22.2222 & . \\
\hline
\end{tabular}

SUITE $=$ Metals Analyte $=\mathrm{ZN}$ Unit $=\mu \mathrm{G} / \mathrm{L}$

\begin{tabular}{|c|c|c|c|c|c|c|c|c|c|}
\hline Location & NM Date & EPA Date & $\begin{array}{l}\text { LANL } \\
\text { Date }\end{array}$ & $\begin{array}{l}\text { NMED } \\
\text { Result }\end{array}$ & $\begin{array}{l}\text { EPA } \\
\text { Result }\end{array}$ & $\begin{array}{l}\text { LANL } \\
\text { Result }\end{array}$ & $\begin{array}{l}\text { NMED vs } \\
\text { EPA }\end{array}$ & $\begin{array}{l}\text { NMED vs } \\
\text { LANL }\end{array}$ & $\begin{array}{l}\text { LANL vs } \\
\text { EPA }\end{array}$ \\
\hline DP SPRING & $09 / 02 / 98$ & . & $09 / 02 / 98$ & $<20$ & 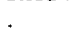 & $<50$ & . & -85.7143 & . \\
\hline MORTANDAD AT GS-1 & $08 / 28 / 98$ & . & $08 / 28 / 98$ & $<20$ & . & $<50$ & . & -85.7143 & . \\
\hline PUEBLO AT SR-502 & $09 / 03 / 98$ & . & $09 / 03 / 98$ & 20 & . & $<50$ & . & -85.7143 & . \\
\hline Location & NM Date & EPA Date & LANL & NMED & EPA & LANL & NMED vs & NMED vs & LANL vs \\
\hline DP SPRING & $09 / 02 / 98$ & & $\begin{array}{l}\text { Date. } \\
09 / 02 / 98\end{array}$ & $\begin{array}{l}\text { Result } \\
51.6\end{array}$ & Result & $\begin{array}{l}\text { Result } \\
68.7850\end{array}$ & EPA & $\begin{array}{l}\text { LANL } \\
-28.5501 *\end{array}$ & EPA \\
\hline
\end{tabular}




\section{APPENDIX C \\ DATA USED IN PAIRED TESTS}

This appendix tabulates the results of the paired difference tests. Paired difference tests (paired t-test and signed-ranks test) consider the direction and magnitude of the differences between paired results (i.e., Is the average, or median, paired difference between Lab 1's results and Lab 2's results significantly different from zero?). The paired t-test involves calculation of the differences between paired data ( $\mathrm{Lab} 1$ minus Lab 2), calculation of the average paired difference, and a statistical test to determine if this average is large enough to suggest different performance of one laboratory compared to the other. The signed-ranks test involves ranking the absolute values of the differences between paired results and counting the number of ranks with positive signs. A statistical test determines if this sum is small enough to indicate a median difference unequal to zero. A paired t-test is run unless the assumption of normality is violated, in which case, a signed ranks test is run.

Results were paired by location and date and differences were calculated only for pairs of detected results.

Difference $=$ Result from Lab $1-$ Result from Lab 2

Chemical concentrations are presented in the unit of $\mu \mathrm{g} / \mathrm{L}$ (micrograms per liter). Radionuclides or radioactivity are presented in the unit of $\mathrm{pCi} / \mathrm{L}$ (picocuries per liter).

The table is organized by:

A. Labs being compared:

1. NMED (Lab 1) vs. EPA (Lab 2)

2. NMED (Lab 1) vs. LANL (Lab 2)

3. NMED (Lab 1) vs. EPA (Lab 2)

B. Sample Size:

1. $N>=5$

2. $\mathrm{N}<5$

C. Analytical Suite

1. Inorganics

2. Metals

3. Organics

4. Rads (Radionuclides and Radioactivity)

D. Sample Type

1. $\mathrm{T}=$ Unfiltered

2. $F=$ Filtered

E. Analyte

A 2-sided paired t-test was used if data were normally distributed (denoted (1) in table), otherwise a signed ranks test was used (denoted (2) in table). Statistically significant results (defined by $\mathrm{p}<0.05$ ) are bolded and italicized. 


\section{Explanation of Column Headers:}

Analyte:

Min.-NMED:

Max.-NMED:

Min.-EPA:

Max.-EPA:

Min.-LANL:

Max.-LANL:

Median-NMED:

Median-EPA:

Median-LANL:

N-Diff:

Min.-Diff:

Max.-Diff:

Mean.-Diff:

Median-Diff:
Analyte under consideration

Minimum result reported for the analyte by NMED

Maximum result reported for the analyte by NMED

Minimum result reported for the analyte by EPA

Maximum result reported for the analyte by EPA

Minimum result reported for the analyte by LANL

Maximum result reported for the analyte by LANL

Median result reported for the analyte by NMED

Median result reported for the analyte by EPA

Median result reported for the analyte by LANL

Number of differences between paired results from Lab 1 and Lab 2, which was calculated for that analyte Minimum difference between paired results from Lab 1 and Lab 2, which was calculated for that analyte

Maximum difference between paired results from Lab 1 and Lab 2, which was calculated for that analyte

Mean difference between paired results from Lab 1 and Lab 2, which was calculated for that analyte Median difference between paired results from Lab 1 and Lab 2, which was calculated for that analyte 
Difference $=$ NMED result - EPA result (Sample Size $>=5$ )

\begin{tabular}{|c|c|c|c|c|c|c|c|c|c|c|c|}
\hline Analyte & Min.-NMED & Max.-NMED & Min.-EPA & Max.-EPA & $\begin{array}{l}\text { Median- } \\
\text { NMED }\end{array}$ & Median-EPA & N-Diff. & Min.-Diff. & Max.-Diff. & Mean-Diff. & Median-Diff. \\
\hline $\mathrm{CL}$ & 2200 & 36400 & 2070.00 & 39800.00 & 20000 & 20800.00 & 7 & -3400.00 & 1200.00 & -1067.14 & -800.00 \\
\hline $\mathrm{F}$ & 130 & 2500 & 160.00 & 3090.00 & 1530 & 1270.00 & 7 & -590.00 & 730.00 & 155.71 & 140.00 \\
\hline NITRATE/NITRITE (l) & 280 & 15000 & 226.00 & 18200.00 & 4900 & 5570.00 & 7 & -3500.00 & 85.00 & -1434.43 & -670.00 \\
\hline SO4 & 2100 & 98000 & 2030.00 & 125000.00 & 16000 & 16400.00 & 7 & -27000.00 & 1080.00 & -3964.29 & -400.00 \\
\hline TDS & 350000 & 560000 & 374000.00 & 538000.00 & 380000 & 404000.00 & 5 & -54000.00 & 22000.00 & -8400.00 & -4000.00 \\
\hline Analyte & Min.-NMED & Max.-NMED & Min.-EPA & Max.-EPA & $\begin{array}{l}\text { Median- } \\
\text { NMED }\end{array}$ & Median-EPA & N-Diff. & Min.-Diff. & Max.-Diff. & Mean-Diff. & Median-Diff. \\
\hline $\mathrm{H}-3$ & 11.000 & 19900.00 & 105,000 & 19200.00 & 13600.00 & 13300.00 & 5 & -400.000 & 700.000 & 141.200 & 200.000 \\
\hline SR-90 & 0.790 & 47.20 & 0.020 & 63.50 & 14.70 & 0.58 & 7 & -31.800 & 19.100 & 2.793 & 1.180 \\
\hline $\mathrm{U}-234$ & 0.350 & 5.67 & 0.590 & 5.62 & 1.03 & 0.75 & 9 & -1.770 & 0.590 & -0.193 & 0.050 \\
\hline U-235 & 0.020 & 0.18 & 0.010 & 0.34 & 0.05 & 0.09 & 8 & -0.163 & 0.090 & -0.062 & -0.053 \\
\hline U-238 & 0.175 & 2.25 & 0.370 & 2.07 & 0.74 & 0.79 & 9 & -0.530 & 0.310 & -0.026 & 0.020 \\
\hline & & & & Differen & NMED res & EPA result (Sa & ple Size & & & & \\
\hline & & & & & te $=$ & cs Type & 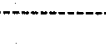 & 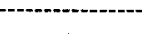 & (1) & & \\
\hline Analyte & Min.-NMED & Max.-NMED & Min.-EPA & Max.-EPA & $\begin{array}{l}\text { Median- } \\
\text { NMED }\end{array}$ & Median-EPA & N-Diff. & Min.-Diff. & Max.-Diff. & Mean-Diff. & Median-Diff. \\
\hline AMMONIA & 11000 & 11000 & 11500.0 & 11500 & 11000 & 11500 & 1 & -500 & -500 & $-500: 00$ & -500.00 \\
\hline TOT. KJELDAHL N & 200 & 15000 & 57.5 & 225000 & 1300 & 145 & 4 & -210000 & 1335 & -51886.88 & 558.75 \\
\hline TSS & 59000 & 59000 & 47000.0 & 47000 & 59000 & 47000 & 1 & 12000 & 12000 & 12000.00 & 12000.00 \\
\hline Analyte & Min.-NMED & Max.-NMED & Min.-EPA & Max.-EPA & $\begin{array}{l}\text { Median- } \\
\text { NMED }\end{array}$ & Median-EPA & N-Diff. & Min.-Diff. & Max,-Diff. & Mean-Diff. & Median-Diff. \\
\hline $\mathrm{AL}$ & 200 & 1600 & 152.000 & 707.000 & 350.0 & 462.000 & 4 & -112.000 & 893.000 & 179.250 & -32.000 \\
\hline AS & 20 & 20 & 2.300 & 2.300 & 20.0 & 2.300 & 1 & 17.700 & 17.700 & 17.700 & 17.700 \\
\hline B & 190 & 190 & 31.900 & 31.900 & 190.0 & 31.900 & 1 & 158.100 & 158.100 & 158.100 & 158.100 \\
\hline $\boldsymbol{B A}(\boldsymbol{I}$ & 45 & 200 & 36.000 & 187.000 & 49.5 & 39.900 & 4 & 6.000 & 13.000 & 10.300 & 11.100 \\
\hline $\mathrm{CU}$ & 110 & 110 & 155.000 & 155.000 & 110.0 & 155.000 & 1 & -45.000 & -45.000 & -45.000 & -45.000 \\
\hline $\mathrm{FE}$ & 100 & 600 & 123.000 & 376.000 & 100.0 & 329.000 & 3 & -229.000 & 224.000 & -9.333 & -23.000 \\
\hline$M N$ & 4 & 4 & 8.300 & 8.300 & 4.0 & 8.300 & 1 & -4.300 & -4.300 & -4.300 & -4.300 \\
\hline MO & 80 & 120 & 117.000 & 126.000 & 100.0 & 118.000 & 3 & -38.000 & -6.000 & -20.333 & -17.000 \\
\hline $\mathrm{PB}$ & 5 & 5 & 4.000 & 4.000 & 5.0 & 4.000 & 1 & 1.000 & 1.000 & 1.000 & 1.000 \\
\hline Analyte & Min.-NMED & Max.-NMED & Min.-EPA & Max.-EPA & $\begin{array}{l}\text { Median- } \\
\text { NMED }\end{array}$ & Median-EPA & N-Diff. & Min.-Diff. & Max.-Diff. & Mean-Diff. & Median-Diff. \\
\hline $\mathrm{AL}$ & 8100 & 8100 & 14900.00 & 14900.00 & 8100 & 14900.00 & 1 & -6800.00 & -6800.00 & -6800.00 & -6800.00 \\
\hline B & 100 & 100 & 179.00 & 179.00 & 100 & 179.00 & 1 & -79.00 & -79.00 & -79.00 & -79.00 \\
\hline BA & 200 & 200 & 173.00 & 173.00 & 200 & 173.00 & 1 & 27.00 & 27.00 & 27.00 & 27.00 \\
\hline FE & 11000 & 11000 & 14900.00 & 14900.00 & 11000 & 14900.00 & 1 & -3900.00 & -3900.00 & -3900.00 & -3900.00 \\
\hline MN & 1300 & 1300 & 13200.00 & 13200.00 & 1300 & 13200.00 & 1 & -11900.00 & -11900.00 & -11900.00 & -11900.00 \\
\hline PB & 3 & 3 & 5.60 & 5.60 & 3 & 5.60 & 1 & -2.60 & -2.60 & -2.60 & -2.60 \\
\hline
\end{tabular}


Analyte

GROSS ALPHA GROSS BETA

\section{Min.-NMED}

160.0

160

160

391
Min.-EPA

133.000

13.200

$\begin{array}{ll}\text { Max.-EPA } & \text { Median- } \\ & \text { NMED } \\ 133 & 160.0 \\ 489 & 202.6\end{array}$

202.6

\section{Median-EPA N-Diff.}

133.000
251.100
Min.-Diff.

27
-98
Max.-Diff.

27.0000

1.0000
Mean-Diff.

27.0000

$-48.5000$
Median-Diff.

Difference $=$ NMED result $-\mathbf{L A N L}$ result $($ Sample Size $<\mathbf{5})$ Suite $=$ Inorganics Type $=\mathrm{T}$

\begin{tabular}{|c|c|c|c|c|c|c|c|c|c|c|}
\hline Min.-NMED & Max.-NMED & Min.-LANL & Max.-LANL & $\begin{array}{l}\text { Median- } \\
\text { NMED }\end{array}$ & Median-LANL & N-Diff. & Min.-Diff. & Max.-Diff. & Mean-Diff. & Median-Diff. \\
\hline 2200 & 2200 & 3600.00 & 3600.00 & 2200 & 3600.00 & 1 & -1400.00 & -1400.00 & -1400.00 & -1400.00 \\
\hline 130 & 130 & 150.00 & 150.00 & 130 & 150.00 & 1 & -20.00 & -20.00 & -20.00 & -20.00 \\
\hline 2100 & 2100 & 3000.00 & 3000.00 & 2100 & 3000.00 & 1 & -900.00 & -900.00 & -900.00 & -900.00 \\
\hline Min.-NMED & Max.-NMED & Min.-LANL & Max.-LANL & $\begin{array}{l}\text { Median- } \\
\text { NMED }\end{array}$ & Median-LANL & N-Diff. & Min.-Diff. & Max.-Diff. & Mean-Diff. & Median-Diff. \\
\hline 1600 & 1600 & 272 & 272 & 1600.0 & 272.0 & 1 & 1328 & 1328 & 1328.00 & 1328 \\
\hline 20 & 20 & 8 & 8 & 20.0 & 8.0 & 1 & 12 & 12 & 12.00 & 12 \\
\hline 360 & 360 & 303 & 303 & 360.0 & 303.0 & 1 & 57 & 57 & 57.00 & 57 \\
\hline 28 & 51 & 20 & 41 & 39.5 & 30.5 & 2 & 8 & 10 & 9.00 & 9 \\
\hline 100 & 100 & 101 & 101 & 100.0 & 101.0 & 1 & -1 & -1 & -1.00 & -1 \\
\hline 600 & 800 & 177 & 593 & 700.0 & 385.0 & 2 & 207 & 423 & 315.00 & 315 \\
\hline 4 & 330 & 5 & 299 & 167.0 & 152.0 & 2 & -1 & 31 & 15.00 & 15 \\
\hline 10 & 10 & 20 & 20 & 10.0 & 20.0 & 1 & -10 & -10 & -10.00 & -10 \\
\hline 70 & 100 & 75 & 97 & 90.0 & 87.0 & 3 & -5 & 3 & 0.33 & 3 \\
\hline
\end{tabular}

$\begin{array}{llllllll}\text { Min.-NMED } & \text { Max.-NMED } & \text { Min.-LANL } & \text { Max.-LANL } & \begin{array}{l}\text { Median- } \\ \text { NMED }\end{array} & \text { Median-LANL } & \text { N-Diff. } & \text { Min.-Diff. } \\ 100.00 & 100.00 & 173.00 & 173.00 & 100.00 & 173.00 & 1 & -73.00 \\ 11000.00 & 11000.00 & 11605.00 & 11605.00 & 11000.00 & 11605.00 & 1 & -605.00 \\ 2000.00 & 2000.00 & 1532.00 & 1532.00 & 2000.00 & 1532.00 & 1 & 468.00 \\ 4000.00 & 4000.00 & 3939.00 & 3939.00 & 4000.00 & 3939.00 & 1 & 61.00 \\ 9000.00 & 9000.00 & 10569.00 & 10569.00 & 9000.00 & 10569.00 & 1 & -1569.00 \\ 120.00 & 120.00 & 114.00 & 123.00 & 120.00 & 118.50 & 2 & -3.00 \\ 1.26 & 1.26 & 0.85 & 0.85 & 1.26 & 0.85 & 1 & 0.41 \\ & & & & & & & \end{array}$

Analyte

Min.-NMED

Max.-NMED

Min -LANL

Max.-LANL Median-

Median-LANL N-Diff.

Min.-Diff.

68.7850
Max.-Diff.

$-73.00$

$-605.00$

61.00

$-1569.00$

6.00

0.41
Mean-Diff

\section{$-73.00$}

$-605.00$

468.00

61.00

$-1569.00$

1.50

0.41
Median-Diff.

$-73.00$

$-605.00$

468.00

61.00

$-1569.00$

1.50

0.41

\section{Max.-Diff. Mean-Diff}

$-17.1850$

Median-Diff

$-17.1850$ 
Difference $=$ NMED result - LANL result $($ Sample Size $<5)$

Analyte

AM-241

GROSS ALPHA

GROSS BETA

H-3

PU-238

PU-239

Analyte

Analyte

CL

NO3/NO2

SO4

TDS

TSS

Analyte
AL
$B(1)$
BA
FE
MN
MO

Ánalyte

AL

$B(1)$

FE

MN

MO

Analyte

GROSS ALPHA(2)

GROSS BETA

SR-90 (2)

$\begin{array}{lll}\text { Min.-NMED } & \text { Max.-NMED } & \text { Min.-LANL } \\ 0.03 & 4.20 & 0.02 \\ 20.90 & 20.90 & 200.41 \\ 479.00 & 479.00 & 294.32 \\ 4640.00 & 19900.00 & 3501.25 \\ 0.00 & 10.70 & -0.03 \\ 0.00 & 2.96 & -0.01 \\ 1.20 & 47.20 & 0.29\end{array}$

$\begin{array}{ll}\text { Max.-LANL } & \text { Median- } \\ & \text { NMED } \\ 24.90 & 0.76 \\ 200.41 & 20.90 \\ 294.32 & 479.00 \\ 19211.25 & 11800.00 \\ 52.02 & 0.35 \\ 16.91 & 0.09 \\ 45.92 & 23.55\end{array}$

Median-LAN
0.70
200.41
294.32
14011.25
0.44
0.11
23.47

Min.-Diff.

$-20.70$

$-179: 51$

184.68

$-2211.25$

$-41.32$

$-13.95$

Difference $=$ LANL result - EPA result (Sample Size >=5)

Min.-LANL
57000.00
3100.00
150.00
280.00
3000.00
144000.00
1000.00

$\begin{array}{ll}\text { Max.-LANL } & \text { Min.-EPA } \\ 109000.00 & 56600.00 \\ 60000.00 & 1980.00 \\ 420.00 & 160.00 \\ 5270.00 & 226.00 \\ 22000.00 & 2030.00 \\ 740000.00 & 125000.00 \\ 49000.00 & 1000.00\end{array}$

$\begin{array}{ll}\text { Max.-EPA } & \text { Median-LANL } \\ 111000.00 & 65000.00 \\ 69900.00 & 3900.00 \\ 350.00 & 200.00 \\ 5570.00 & 400.00 \\ 23200.00 & 4000.00 \\ 777000.00 & 238000.00 \\ 63000.00 & 5000.00\end{array}$

Median-EPA
74700.00
3150.00
240.00
569.00
2920.00
162000.00
9000.00

N-Diff.
5
5
5
5
5
5
13

Min.-Diff. $-23000.00$ $-9900.00$$$
-40.00
$$

$-367.00$

-1820.00
-473000.00

$-14000.00$

Suite $=$ Metals Type $=$

Min.-LANL
61
43
35
54
3
68

$\begin{array}{lll}\text { Max.-LANL } & \text { Min.-EPA } & \text { Max.-EPA } \\ 495 & 61.0000 & 707 \\ \mathbf{2 7 3} & \mathbf{5 7 . 0 0 0 0} & \mathbf{3 1 4} \\ 148 & 40.2000 & 146 \\ 455 & 5.0000 & 452 \\ 967 & 0.9100 & 942 \\ 328 & \mathbf{5 8 . 5 0 0 0} & 317\end{array}$

$\begin{array}{ll}\text { Median-LANL } & \text { Median-EPA } \\ 260.0 & 158.0 \\ 77.0 & 114.0 \\ 67.5 & 62.5 \\ 153.5 & 125.0 \\ 5.0 & 3.0 \\ 141.0 & 138.0\end{array}$

N-Diff.
8
11
12
8
5
6

$\begin{array}{ll}\text { Min.-Diff. } & \text { Max.-Diff. } \\ -435.000 & 326.000 \\ -62.000 & \mathbf{3 . 0 0 0} \\ -5.200 & 8.200 \\ -203.000 & 245.000 \\ -3.300 & 25.000 \\ -5.000 & 19.000\end{array}$

$-3.8750$

$\mathbf{- 3 5 . 8 0 9 1}$

1.6333

5.4500

7.1580

6.5833

$\begin{array}{ll}\text { Max.-LANL } & \text { Min.-EPA } \\ 1200 & 65.800 \\ \mathbf{2 9 0} & \mathbf{3 6 . 6 0 0} \\ \mathbf{1 5 0} & \mathbf{7 . 0 0 0} \\ 21405 & 44.100 \\ 1008 & 2.400 \\ 335 & 113.000\end{array}$

Max.-EPA
746
$\mathbf{3 3 4}$
$\mathbf{1 4 7}$
21300
925
315

$\begin{array}{ll}\text { Median-LANL } & \text { Median-EPA } \\ \mathbf{3 5 9 . 0} & 247.000 \\ \mathbf{6 5 . 5} & \mathbf{1 0 7 . 0 0 0} \\ \mathbf{6 0 . 0} & \mathbf{5 6 . 3 0 0} \\ 333.0 & 212.500 \\ 8.5 & 7.600 \\ 158.0 & 151.000\end{array}$

N-Diff.
7
12
14
12
10
5

Min.-Diff.
$-\mathbf{3 2 3 . 0 0 0}$
$\mathbf{- 5 0 . 0 0 0}$
$\mathbf{- 3 . 8 0 0}$
-312.000
-7.000
-3.000

Max.Diff

839.00

$-1.80$

12.60

1857.90

83.00

- Suite $=$ Rads Type $=T$

$\mathbf{- 5 . 0 2 7 2}$

. .2875
.98 .7500

-98.7500
-0.0250

$\begin{array}{ll}\text { Max.-LANL } & \text { Min.-EPA } \\ 8.09 & 1.07000 \\ 264.32 & 2.28000 \\ 19211.25 & \mathbf{2 . 0 7 0 0 0} \\ \mathbf{4 8 . 3 8} & -0.15000\end{array}$

Max.-EPA
19.70
148.00
19200.00
$\mathbf{3 2 . 4 0}$

$\begin{array}{ll}\text { Median-LANL } & \text { Median-EPA } \\ \boldsymbol{0 . 1 9 8} & \mathbf{5 . 0 7 0} \\ 17.312 & 23.300 \\ \mathbf{4 0 1 . 2 5 0} & \mathbf{1 4 5 . 0 0 0} \\ \mathbf{0 . 5 4 0} & \mathbf{0 . 6 2 5}\end{array}$

N-Diff.
14
14
18
14

Min.-Diff.

$-17.73$

$-58.58$

$-1388.75$

.0 .84

$\begin{array}{ll}\text { Max.-Diff, } & \text { Mean-Diff. } \\ \mathbf{- 0 . 5 0} & \mathbf{- 6 . 6 6 7} \\ 146.32 & 6.499 \\ \mathbf{3 9 9 1 . 2 5} & \mathbf{5 4 8 . 7 2 7} \\ \mathbf{1 8 . 6 8} & \mathbf{5 . 5 6 5}\end{array}$

Median-Diff.

$-0.033$

$-179.513$

184.682

688.750

$-0.025$

0.960 
Difference $=$ LANL result - EPA result (Sample Size $<$ 5)

\begin{tabular}{|c|c|c|c|c|c|c|c|c|c|c|c|}
\hline $\begin{array}{l}\text { Analyte } \\
\text { AS }\end{array}$ & $\begin{array}{l}\text { Min.-LANL } \\
2\end{array}$ & $\begin{array}{l}\text { Max.-LANL } \\
7\end{array}$ & $\begin{array}{l}\text { Min.-EPA } \\
2.0000\end{array}$ & $\begin{array}{l}\text { Max.-EPA } \\
6.6000\end{array}$ & $\begin{array}{l}\text { Median-LANL } \\
3.5\end{array}$ & $\begin{array}{l}\text { Median-EPA } \\
3.4500\end{array}$ & $\begin{array}{l}\text { N-Diff. } \\
4\end{array}$ & $\begin{array}{l}\text { Min.-Diff. } \\
-0.2000\end{array}$ & $\begin{array}{l}\text { Max.-Diff. } \\
0.4000\end{array}$ & $\begin{array}{l}\text { Mean-Diff. } \\
0.1250\end{array}$ & $\begin{array}{l}\text { Median-Diff. } \\
0.1500\end{array}$ \\
\hline $\mathrm{CU}$ & 15 & 15 & 17.0000 & 17.0000 & 15.0 & 17.0000 & 1 & -2.0000 & -2.0000 & -2.0000 & -2.0000 \\
\hline PB & 61 & 61 & 3.2000 & 3.2000 & 61.0 & 3.2000 & 1 & 57.8000 & 57.8000 & 57.8000 & 57.8000 \\
\hline Analyte & Min.-LANL & Max.-LANL & Min.-EPA & Max.-EPA & Median-LANL & Median-EPA & N-Diff. & Min.-Diff. & Max.-Diff. & Mean-Diff. & Median-Diff. \\
\hline & 7 & 7 & 5.3000 & 5.3000 & 7.0 & 5.3000 & 1 & 1.70000 & 1.7000 & 1.7000 & 1.7000 \\
\hline $\mathrm{CU}$ & 18 & 31 & 13.0000 & 14.9000 & 24.5 & 13.9500 & 2 & 3.10000 & 18.0000 & 10.5500 & 10.5500 \\
\hline PB & 5 & 97 & 4.0000 & 28.4000 & 49.5 & 11.7500 & 4 & 0.90000 & 93.0000 & 36.2750 & 25.6000 \\
\hline $\begin{array}{l}\text { Analyte } \\
\text { ACETONE }\end{array}$ & $\begin{array}{l}\text { Min.-LANL } \\
86\end{array}$ & $\begin{array}{l}\text { Max.-LANL } \\
86\end{array}$ & $\begin{array}{l}\text { Min.-EPA } \\
12\end{array}$ & $\begin{array}{l}\text { Max.-EPA } \\
12\end{array}$ & $\begin{array}{l}\text { Median-LANL } \\
86\end{array}$ & $\begin{array}{l}\text { Median-EPA } \\
12\end{array}$ & $\begin{array}{l}\text { N-Diff. } \\
1\end{array}$ & $\begin{array}{l}\text { Min.-Diff. } \\
74\end{array}$ & $\begin{array}{l}\text { Max.-Diff. } \\
74\end{array}$ & $\begin{array}{l}\text { Mean-Diff. } \\
74\end{array}$ & $\begin{array}{l}\text { Median-Diff. } \\
74\end{array}$ \\
\hline \multicolumn{12}{|c|}{ Difference $=$ NMED result - EPA result $($ Sample Size $>=5$ ) } \\
\hline Analyte & Min.-NMED & Max.-NMED & Min.-EPA & Max.-EPA & $\begin{array}{l}\text { Median- } \\
\text { NMED }\end{array}$ & Median-EPA & N-Diff. & Mị.-Diff. & Max.-Diff. & Mean-Diff. & Median-Diff. \\
\hline CL & 2200 & 36400 & 2070.00 & 39800.00 & 20000 & 20800.00 & 7 & -3400.00 & 1200.00 & -1067.14 & -800.00 \\
\hline $\mathbf{F}$ & 130 & 2500 & 160.00 & 3090.00 & 1530 & 1270.00 & 7 & .590 .00 & 730.00 & 155.71 & 140.00 \\
\hline NITRATE/NITRITE (l) & 280 & 15000 & 226.00 & 18200.00 & 4900 & 5570.00 & 7 & -3500.00 & 85.00 & -1434.43 & -670.00 \\
\hline SO4 & 2100 & 98000 & 2030.00 & 125000.00 & 16000 & 16400.00 & 7 & -27000.00 & 1080.00 & -3964.29 & -400.00 \\
\hline TDS & 350000 & 560000 & 374000.00 & 538000.00 & 380000 & 404000.00 & 5 & -54000.00 & 22000.00 & -8400.00 & -4000.00 \\
\hline Analyte & Min.-NMED & Max.-NMED & Min.-EPA & Max.-EPA & $\begin{array}{l}\text { Median- } \\
\text { NMED }\end{array}$ & Median-EPA & N-Diff. & Min.-Diff. & Max.-Diff. & Mean-Diff. & Median-Diff. \\
\hline $\mathrm{H}-3$ & 11.000 & 19900.00 & 105.000 & 19200.00 & 13600.00 & 13300.00 & 5 & -400.000 & 700.000 & 141.200 & 200.000 \\
\hline SR-90 & 0.790 & 47.20 & 0.020 & 63.50 & 14.70 & 0.58 & 7 & -31.800 & 19.100 & 2.793 & 1.180 \\
\hline U-234 & 0.350 & 5.67 & 0.590 & 5.62 & 1.03 & 0.75 & 9 & -1.770 & 0.590 & -0.193 & 0.050 \\
\hline U-235 & 0.020 & 0.18 & 0.010 & 0.34 & 0.05 & 0.09 & 8 & -0.163 & 0.090 & -0.062 & -0.053 \\
\hline U-238 & 0.175 & 2.25 & 0.370 & 2.07 & 0.74 & 0.79 & 9 & -0.530 & 0.310 & -0.026 & 0.020 \\
\hline \multicolumn{12}{|c|}{ Difference $=$ NMED result - EPA result $($ Sample Size $<5)$} \\
\hline Analyte & Min.-NMED & Max.-NMED & Min.-EPA & Max.-EPA & $\begin{array}{l}\text { Median- } \\
\text { NMED }\end{array}$ & Median-EPA & N-Diff. & Min.-Diff. & Max.-Diff. & Mean-Diff. & Median-Diff. \\
\hline AMMONIA & 11000 & 11000 & 11500.0 & 11500 & 11000 & 11500 & 1 & -500 & -500 & -500.00 & -500.00 \\
\hline TOT. KJELDAHL N & 200 & 15000 & 57.5 & 225000 & 1300 & 145 & 4 & -210000 & 1335 & -51886.88 & 558.75 \\
\hline TSS & 59000 & 59000 & 47000.0 & 47000 & 59000 & 47000 & 1 & 12000 & 12000 & 12000.00 & 12000.00 \\
\hline
\end{tabular}




\begin{tabular}{|c|c|c|c|c|c|c|c|c|c|c|c|}
\hline Analyte & Min.-NMED & Max.-NMED & Min.-EPA & Max.-EPA & $\begin{array}{l}\text { Median- } \\
\text { NMED }\end{array}$ & Median-EPA & N-Diff. & Min.-Diff. & Max.-Diff. & Mean-Diff. & Median-Diff. \\
\hline AL & 200 & 1600 & 152.000 & 707.000 & 350.0 & 462.000 & 4 & -112.000 & 893.000 & 179.250 & -32.000 \\
\hline AS & 20 & 20 & 2.300 & 2.300 & 20.0 & 2.300 & 1 & 17.700 & 17.700 & 17.700 & 17.700 \\
\hline B & 190 & 190 & 31.900 & 31.900 & 190.0 & 31.900 & 1 & 158.100 & 158.100 & 158.100 & 158,100 \\
\hline$B A(I)$ & 45 & 200 & 36.000 & 187.000 & 49.5 & 39.900 & 4 & 6.000 & 13.000 & 10.300 & 11.100 \\
\hline $\mathrm{CU}$ & 110 & 110 & 155.000 & 155.000 & 110.0 & 155.000 & 1 & -45.000 & -45.000 & .45 .000 & -45.000 \\
\hline FE & 100 & 600 & 123.000 & 376.000 & 100.0 & 329.000 & 3 & -229.000 & 224.000 & -9.333 & -23.000 \\
\hline $\mathrm{MN}$ & 4 & 4 & 8.300 & $8: 300$ & 4.0 & 8.300 & 1 & -4.300 & -4.300 & -4.300 & -4.300 \\
\hline MO & 80 & 120 & 117.000 & 126.000 & 100.0 & 118.000 & 3 & -38.000 & -6.000 & -20.333 & -17.000 \\
\hline PB & 5 & 5 & 4.000 & 4.000 & 5.0 & 4.000 & 1 & 1.000 & 1.000 & 1.000 & 1.000 \\
\hline
\end{tabular}

\begin{tabular}{|c|c|c|c|c|c|c|c|c|c|c|c|}
\hline Analyte & Min.-NMED & Max.-NMED & Min.-EPA & Max.-EPA & $\begin{array}{l}\text { Median- } \\
\text { NMED }\end{array}$ & Median-EPA & N-Diff. & Min.-Diff. & Max.-Diff. & Mean-Diff. & Median-Diff. \\
\hline $\mathrm{AL}$ & 8100 & 8100 & 14900.00 & 14900.00 & 8100 & 14900.00 & 1 & -6800.00 & -6800.00 & -6800.00 & -6800.00 \\
\hline B & 100 & 100 & 179.00 & 179.00 & 100 & 179.00 & 1 & -79.00 & -79.00 & .79 .00 & -79.00 \\
\hline BA & 200 & 200 & 173.00 & 173.00 & 200 & 173.00 & 1 & 27.00 & 27.00 & 27.00 & 27.00 \\
\hline FE & 11000 & 11000 & 14900.00 & 14900.00 & 11000 & 14900.00 & 1 & -3900.00 & -3900.00 & -3900.00 & -3900.00 \\
\hline MN & 1300 & 1300 & 13200.00 & 13200.00 & 1300 & 13200.00 & 1 & -11900.00 & -11900.00 & -11900.00 & -11900.00 \\
\hline PB & 3 & 3 & 5.60 & 5.60 & 3 & 5.60 & 1 & -2.60 & -2.60 & -2.60 & -2.60 \\
\hline Analyte & Min.-NMED & Max.-NMED & Min.-EPA & Max.-EPA & $\begin{array}{l}\text { Median- } \\
\text { NMED }\end{array}$ & Median-EPA & N-Diff. & Min.-Diff. & Max.-Diff. & Mean-Diff. & Median-Diff. \\
\hline GROSS ALPHA & 160.0 & 160 & 133.000 & 133 & 160.0 & 133.000 & 1 & 27 & 27.0000 & 27.0000 & 27.0000 \\
\hline GROSS BETA & 14.2 & 391 & 13.200 & 489 & 202.6 & 251.100 & 2 & -98 & 1.0000 & -48.5000 & -48.5000 \\
\hline
\end{tabular}

Difference $=$ NMED result $\mathbf{~}$ LANL result $($ Sample Size $<$ 5)

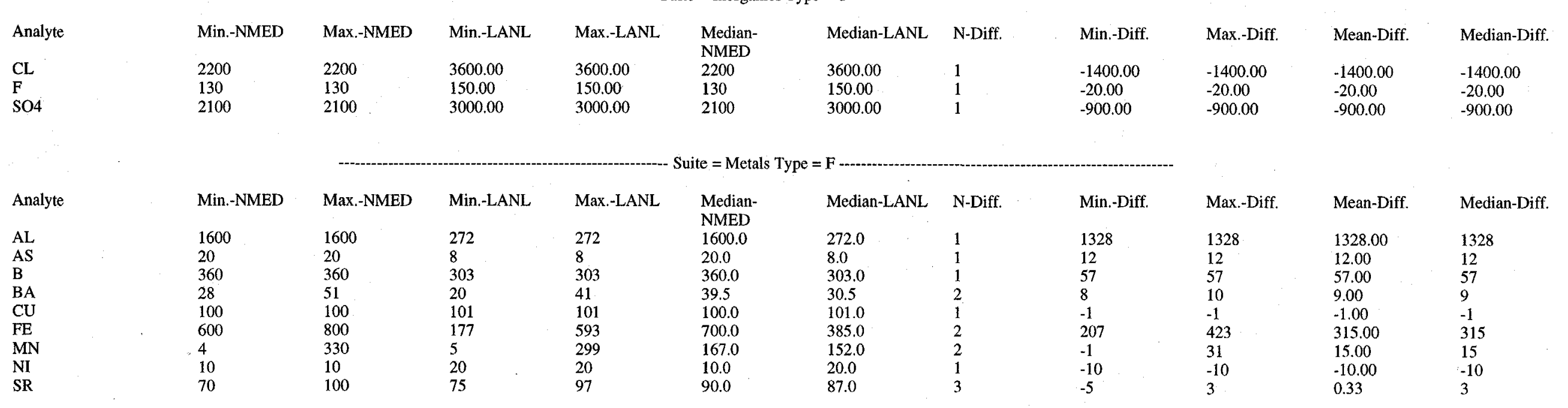




\begin{tabular}{|c|c|c|c|c|c|c|c|c|c|c|c|}
\hline Analyte & Min.-NMED & Max.-NMED & Min.-LANL & Max.-LANL & $\begin{array}{l}\text { Median- } \\
\text { NMED }\end{array}$ & Median-LANL & N-Diff. & Min.-Diff. & Max.-Diff. & Mean-Diff. & Median-Diff. \\
\hline $\mathbf{B}$ & 100.00 & 100.00 & 173.00 & 173.00 & 100.00 & 173.00 & 1 & -73.00 & -73.00 & -73.00 & -73.00 \\
\hline $\mathrm{CA}$ & 11000.00 & 11000.00 & 11605.00 & 11605.00 & 11000.00 & 11605.00 & 1 & -605.00 & -605.00 & -605.00 & -605.00 \\
\hline $\mathbf{K}$ & 2000.00 & 2000.00 & 1532.00 & 1532.00 & 2000.00 & 1532.00 & 1 & 468.00 & 468.00 & 468.00 & 468.00 \\
\hline MG & 4000.00 & 4000.00 & 3939.00 & 3939.00 & 4000.00 & 3939.00 & 1 & 61.00 & 61.00 & 61.00 & 61.00 \\
\hline NA & 9000.00 & 9000.00 & 10569.00 & 10569.00 & 9000.00 & 10569.00 & 1 & -1569.00 & -1569.00 & -1569.00 & -1569.00 \\
\hline SR & 120.00 & 120.00 & 114.00 & 123.00 & 120.00 & 118.50 & 2 & -3.00 & 6.00 & 1.50 & 1.50 \\
\hline URANIUM & 1.26 & 1.26 & 0.85 & 0.85 & 1.26 & 0.85 & 1 & 0.41 & 0.41 & 0.41 & 0.41 \\
\hline Analyte & Min,-NMED & Max.-NMED & Min.-LANL & Max.-LANL & Median- & Median-LANL & N-Diff. & Min.-Diff. & Max.-Diff. & Mean-Diff. & Median-Diff. \\
\hline SR-90 & 51.6 & 51.6 & 68.7850 & 68.7850 & $\begin{array}{l}\text { NIMED } \\
51.6\end{array}$ & 68.7850 & 1 & -17.1850 & -17.1850 & -17.1850 & -17.1850 \\
\hline \multicolumn{12}{|c|}{ Difference $=$ NMED result - LANL result $($ Sample Size $<5)$} \\
\hline Analyte & Min.-NMED & Max.-NMED & Min.-LANL & Max.-LANL & $\begin{array}{l}\text { Median- } \\
\text { NMED }\end{array}$ & Median-LANL & N-Diff. & Min.-Diff. & Max.-Diff. & Mean-Diff. & Median-Diff. \\
\hline AM-241 & 0.03 & 4.20 & 0.02 & 24.90 & 0.76 & 0.70 & 4 & -20.70 & 0.19 & -5.144 & -0.033 \\
\hline GROSS ALPHA & 20.90 & 20.90 & 200.41 & 200.41 & 20.90 & 200.41 & 1 & -179.51 & -179.51 & -179.513 & -179.513 \\
\hline GROSS BETA & 479.00 & 479.00 & 294.32 & 294.32 & 479.00 & 294.32 & 1 & 184.68 & 184.68 & 184.682 & 184.682 \\
\hline H-3 & 4640.00 & 19900.00 & 3501.25 & 19211.25 & 11800.00 & 14011.25 & 3 & -2211.25 & 1138.75 & -127.917 & 688.750 \\
\hline PU-238 & 0.00 & 10.70 & -0.03 & 52.02 & 0.35 & 0.44 & 4 & -41.32 & 0.03 & -10.363 & -0.082 \\
\hline PU-239 & 0.00 & 2.96 & -0.01 & 16.91 & 0.09 & 0.11 & 4 & -13.95 & 0.02 & -3.496 & -0.025 \\
\hline SR-90 & 1.20 & 47.20 & 0.29 & 45.92 & 23.55 & 23.47 & 4 & -0.85 & 1.28 & 0.585 & 0.960 \\
\hline \multicolumn{12}{|c|}{ Difference $=$ LANL result - EPA result (Sample Size $>=5$ ) } \\
\hline Analyte & Min.-LANL & Max.-LANL & Min.-EPA & Max.-EPA & Median-LANL & Median-EPA & N-Diff. & Min.-Diff. & Max.-Diff. & Mean-Diff. & Median-Diff. \\
\hline ALKALINITY & 57000.00 & 109000.00 & 56600.00 & 111000.00 & 65000.00 & 74700.00 & 5 & -23000.00 & 400.00 & -8600.00 & -8700.00 \\
\hline CL & 3100.00 & 60000.00 & 1980.00 & 69900.00 & 3900.00 & 3150.00 & 5 & -9900.00 & 1530.00 & -1760.00 & 750.00 \\
\hline F & 150.00 & 420.00 & 160.00 & 350.00 & 200.00 & 240.00 & 5 & -40.00 & 70.00 & 6.00 & 0.00 \\
\hline NO3/NO2 & 280.00 & 5270.00 & 226.00 & 5570.00 & 400.00 & 569.00 & 5 & -367.00 & 81.00 & -99.40 & 35.00 \\
\hline $\mathrm{SO} 4$ & 3000.00 & 22000.00 & 2030.00 & 23200.00 & 4000.00 & 2920.00 & 5 & -1820.00 & 1080.00 & -12.00 & 910.00 \\
\hline TDS & 144000.00 & 740000.00 & 125000.00 & 777000.00 & 238000.00 & 162000.00 & 5 & -473000.00 & 520000.00 & 43400.00 & 76000.00 \\
\hline TSS & 1000.00 & 49000.00 & 1000.00 & 63000.00 & 5000.00 & 9000.00 & 13 & -14000.00 & 8000.00 & -2692.31 & -3000.00 \\
\hline Analyte & Min.-LANL & Max.-LANL & Min.-EPA & Max.-EPA & Median-LANL & Median-EPA & N-Diff. & Min.-Diff. & Max.-Diff. & Mean-Diff. & Median-Diff. \\
\hline & 61 & 495 & 61.0000 & 707 & 260.0 & 158.0 & 8 & -435.000 & 326.000 & -3.8750 & 0.1000 \\
\hline$B(I)$ & 43 & 273 & 57.0000 & 314 & 77.0 & 114.0 & 11 & -62.000 & 3.000 & -35.8091 & -41.0000 \\
\hline $\mathrm{BA}$ & 35 & 148 & 40.2000 & 146 & 67.5 & 62.5 & 12 & -5.200 & 8.200 & 1.6333 & 1.8000 \\
\hline $\mathrm{FE}$ & 54 & 455 & 5.0000 & 452 & 153.5 & 125.0 & 8 & -203.000 & 245.000 & 5.4500 & 2.0000 \\
\hline MN & 3 & 967 & 0.9100 & 942 & 5.0 & 3.0 & 5 & -3.300 & 25.000 & 7.1580 & 3.0900 \\
\hline MO & 68 & 328 & 58.5000 & 317 & 141.0 & 138.0 & 6 & -5.000 & 19.000 & 6.5833 & 6.7500 \\
\hline
\end{tabular}


Suite $=$ Metals Type $=\mathrm{T}$

\begin{tabular}{|c|c|c|c|c|c|c|c|c|c|c|c|}
\hline Analyte & Min.-LANL & Max.-LANL & Min.-EPA & Max.-EPA & Median-LANL & Median-EPA & N-Diff. & Min,-Diff. & Max.-Diff. & Mean-Diff. & Median-Diff. \\
\hline & 180 & 1200 & 65.800 & & 359.0 & 247.000 & & -323.000 & 839.00 & 120.600 & 147.000 \\
\hline$B(I)$ & 33 & 290 & 36.600 & 334 & 65.5 & 107.000 & 12 & -50.000 & -1.80 & -22.750 & -21.050 \\
\hline$B A(I)$ & 8 & 150 & 7.000 & 147 & 60.0 & 56.300 & 14 & -3.800 & 12.60 & 3.879 & 2.450 \\
\hline $\mathrm{FE}$ & 73 & 21405 & 44.100 & 21300 & 333.0 & 212.500 & 12 & -312.000 & 1857.90 & 262.142 & 99.400 \\
\hline MN & 2 & 1008 & 2.400 & 925 & 8.5 & 7.600 & 10 & -7.000 & 83.00 & 10.560 & 0.000 \\
\hline MO & 121 & 335 & 113.000 & 315 & 158.0 & 151.000 & 5 & -3.000 & 20.00 & 10.200 & 14.000 \\
\hline Analyte & Min-LANL & Max.-LANL & Min.-EPA & Max.-EPA & Median-LANL & Median-EPA & N-Diff. & Min.-Diff. & Max.-Diff. & Mean-Diff. & Median-Diff. \\
\hline $\begin{array}{l}\text { GROSS ALPHA(2) } \\
\text { GROSS BETA }\end{array}$ & -5.0272 & $\begin{array}{l}8.09 \\
26432\end{array}$ & 1.07000 & 19.70 & 0.198 & 5.070 & 14 & .17 .73 & -0.50 & -6.667 & -4.112 \\
\hline $\begin{array}{l}\text { GROSS BETA } \\
H-3(2)\end{array}$ & $\begin{array}{l}1.2875 \\
.98 .7500\end{array}$ & $\begin{array}{l}264.32 \\
19211.25\end{array}$ & $\begin{array}{l}2.28000 \\
2.07000\end{array}$ & $\begin{array}{l}148.00 \\
19200.00\end{array}$ & 17.312 & 23.300 & 14 & -58.58 & 146.32 & 6.499 & -3.667 \\
\hline $\begin{array}{l}H-3(2) \\
S R-90(2)\end{array}$ & $\begin{array}{l}-98.7500 \\
-0.0250\end{array}$ & $\begin{array}{l}19211.25 \\
48.38\end{array}$ & $\begin{array}{l}2.07000 \\
-0.15000\end{array}$ & $\begin{array}{l}19200.00 \\
32.40\end{array}$ & $\begin{array}{l}401.250 \\
0.540\end{array}$ & $\begin{array}{l}145.000 \\
0.625\end{array}$ & $\begin{array}{l}18 \\
14\end{array}$ & $\begin{array}{l}-1388.75 \\
-0.84\end{array}$ & $\begin{array}{l}3991.25 \\
18.68\end{array}$ & $\begin{array}{l}548.727 \\
5565\end{array}$ & $\begin{array}{l}240.315 \\
0.627\end{array}$ \\
\hline
\end{tabular}

Difference = LANL result - EPA result $($ Sample Size <5)

\begin{tabular}{|c|c|c|c|c|c|c|c|c|c|}
\hline $\begin{array}{l}\text { Max.-LANL } \\
7 \\
15 \\
61\end{array}$ & $\begin{array}{l}\text { Min.-EPA } \\
2.0000 \\
17.0000 \\
3.2000\end{array}$ & $\begin{array}{l}\text { Max.-EPA } \\
6.6000 \\
17.0000 \\
3.2000\end{array}$ & $\begin{array}{l}\text { Median-LANL } \\
3.5 \\
15.0 \\
61.0\end{array}$ & $\begin{array}{l}\text { Median-EPA } \\
3.4500 \\
17.0000 \\
3.2000\end{array}$ & $\begin{array}{l}\text { N-Diff, } \\
4 \\
1 \\
1\end{array}$ & $\begin{array}{l}\text { Min.-Diff. } \\
-0.2000 \\
-2.0000 \\
57.8000\end{array}$ & $\begin{array}{l}\text { Max.-Diff. } \\
0.4000 \\
-2.0000 \\
57.8000\end{array}$ & $\begin{array}{l}\text { Mean-Diff. } \\
0.1250 \\
-2.0000 \\
57.8000\end{array}$ & $\begin{array}{l}\text { Median-Diff } \\
0.1500 \\
-2.0000 \\
57.8000\end{array}$ \\
\hline $\begin{array}{l}\text { Max.-LANL } \\
7 \\
31 \\
97\end{array}$ & $\begin{array}{l}\text { Min.-EPA } \\
5.3000 \\
13.0000 \\
4.0000\end{array}$ & $\begin{array}{l}\text { Max.-EPA } \\
5.3000 \\
14.9000 \\
28.4000\end{array}$ & $\begin{array}{l}\text { Median-LANL } \\
7.0 \\
24.5 \\
49.5\end{array}$ & $\begin{array}{l}\text { Median-EPA } \\
5.3000 \\
13.9500 \\
11.7500\end{array}$ & $\begin{array}{l}\text { N-Diff. } \\
1 \\
2 \\
4\end{array}$ & $\begin{array}{l}\text { Min.-Diff. } \\
1.70000 \\
3.10000 \\
0.90000\end{array}$ & $\begin{array}{l}\text { Max.-Diff. } \\
1.7000 \\
18.0000 \\
93.0000\end{array}$ & $\begin{array}{l}\text { Mean-Diff. } \\
1.7000 \\
10.5500 \\
36.2750\end{array}$ & $\begin{array}{l}\text { Median-Diff } \\
1.7000 \\
10.5500 \\
25.6000\end{array}$ \\
\hline $\begin{array}{l}\text { Max.-LANL } \\
86\end{array}$ & $\begin{array}{l}\text { Min.-EPA } \\
12\end{array}$ & $\begin{array}{l}\text { Max.-EPA } \\
12\end{array}$ & $\begin{array}{l}\text { Median-LANL } \\
86\end{array}$ & $\begin{array}{l}\text { Median-EPA } \\
12\end{array}$ & $\begin{array}{l}\text { N-Diff, } \\
1\end{array}$ & $\begin{array}{l}\text { Min.-Diff. } \\
74\end{array}$ & $\begin{array}{l}\text { Max.-Diff. } \\
74\end{array}$ & $\begin{array}{l}\text { Mean-Diff. } \\
74\end{array}$ & $\begin{array}{l}\text { Median-Diff } \\
74\end{array}$ \\
\hline
\end{tabular}




\section{APPENDIX D \\ PLOTS OF DATA USED IN REGRESSION ANALYSES}

Plots of paired data used in the regression analyses are presented in this Appendix. A reference line is provided to show the line which has a slope of 1 and an intercept of $(0,0)$. Points falling on this line indicate prefect agreement between paired results. Actual data pairs are plotted with symbols indicating whether the results came from filtered or unfiltered samples. If the regression analysis showed a statistically significant relationship between filtered and/or unfiltered results, these lines are also shown on the plot. 
Inorganics: LANL vs. EPA
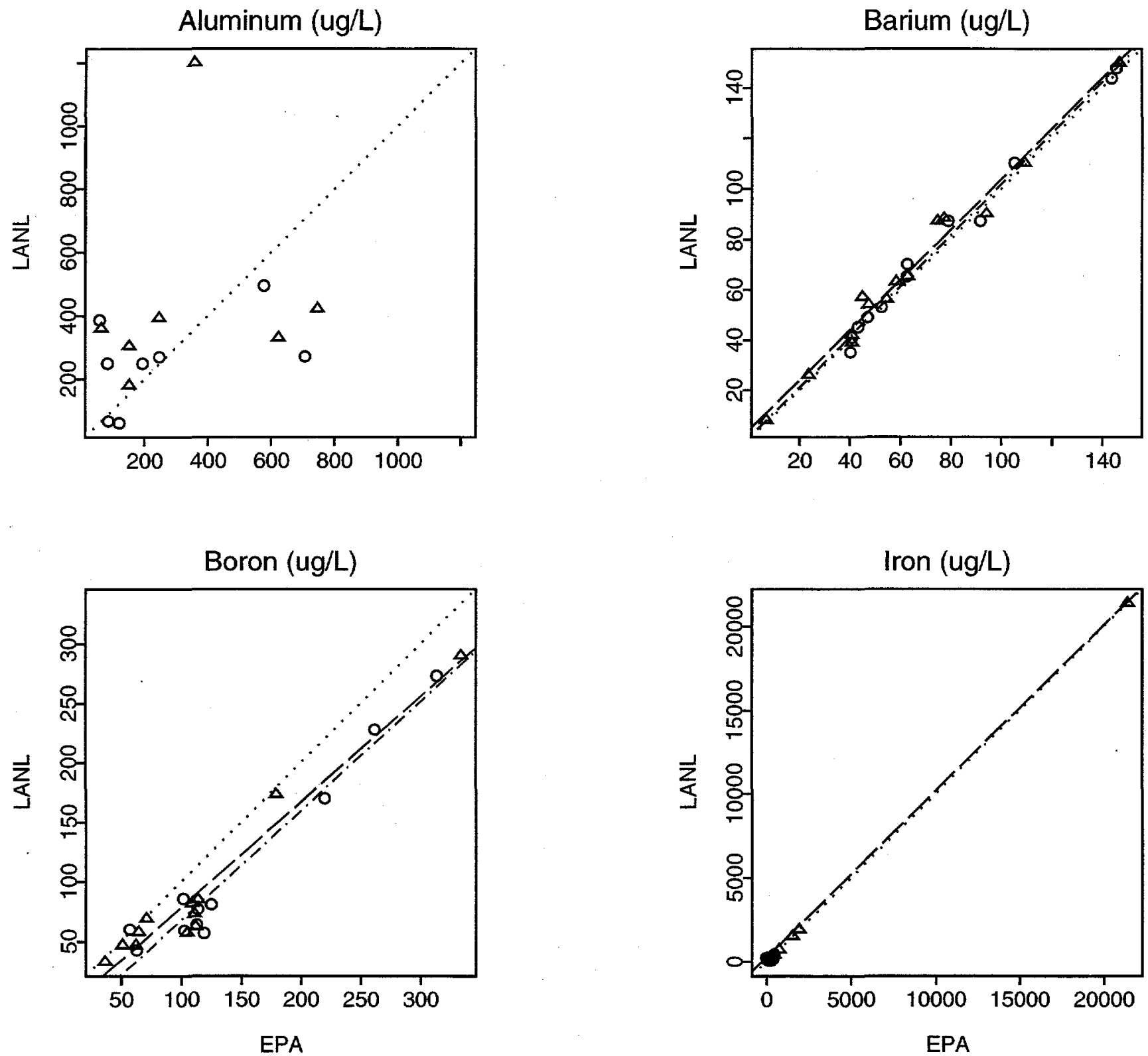

..... Reference Line

-...- Filtered regression

- $\quad$ Filtered samples

- Unfiltered regression

$\Delta \quad$ Unfiltered samples Missing line = no regression relationship 
Inorganics: LANL vs. EPA
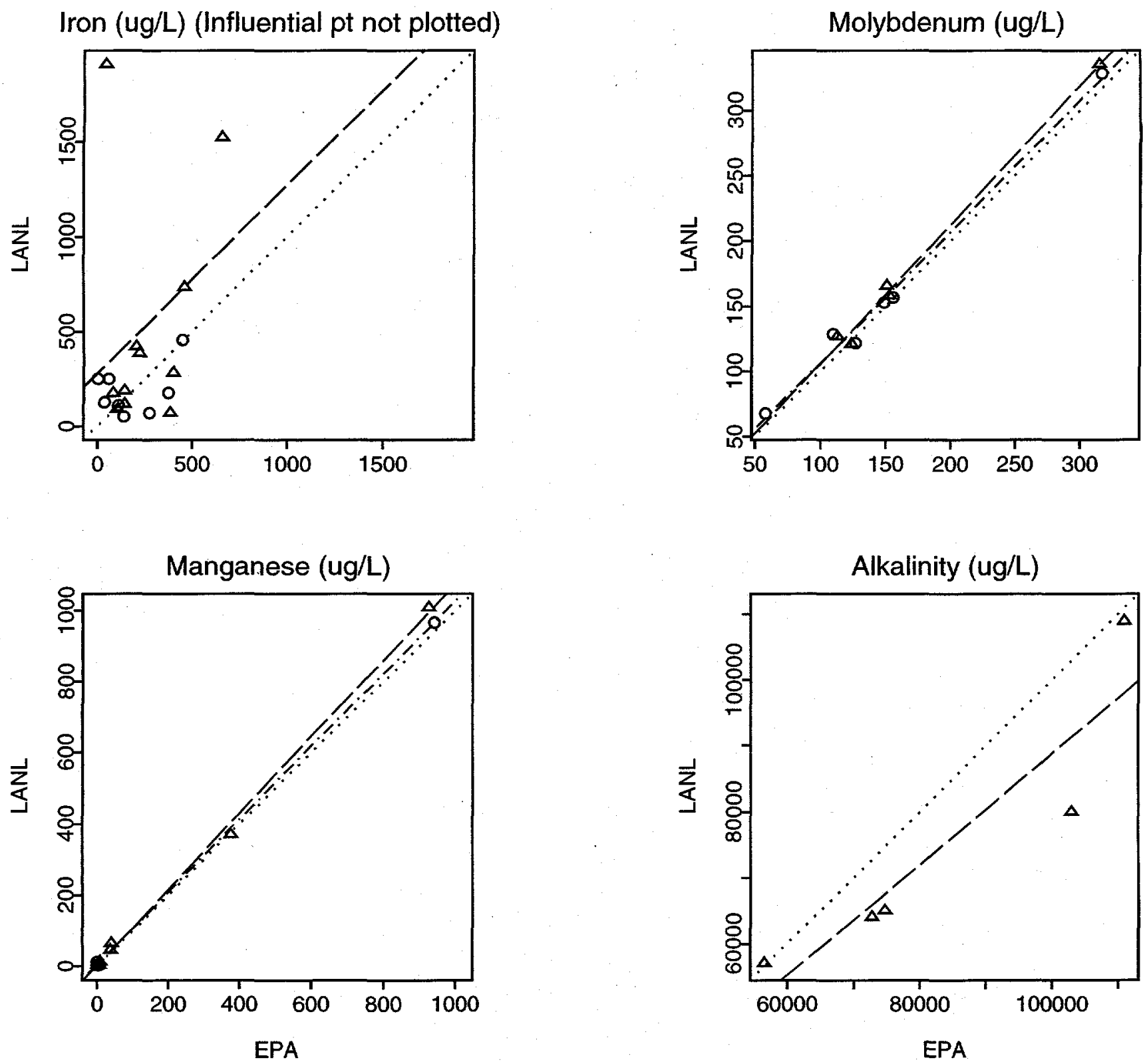

..... Reference Line

-..- Filtered regression

_- Unfiltered regression $\Delta \quad$ Unfiltered samples Missing line = no regression relationship 
Inorganics: LANL vs. EPA
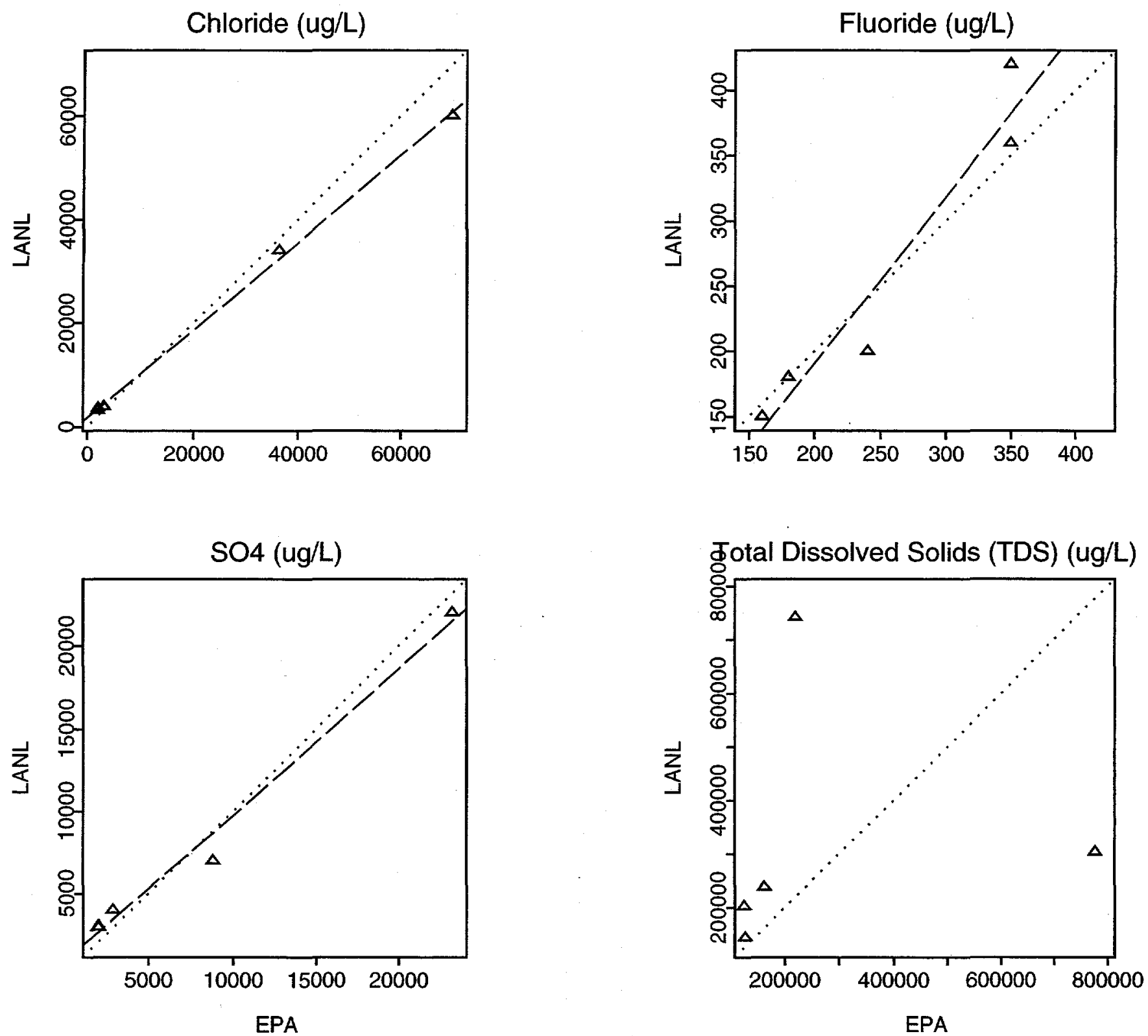

..... Reference Line

-... Filtered regression

- $\quad$ Filtered samples

- Unfiltered regression Missing line =

$\Delta \quad$ Unfiltered samples no regression relationship 
Inorganics: LANL vs. EPA
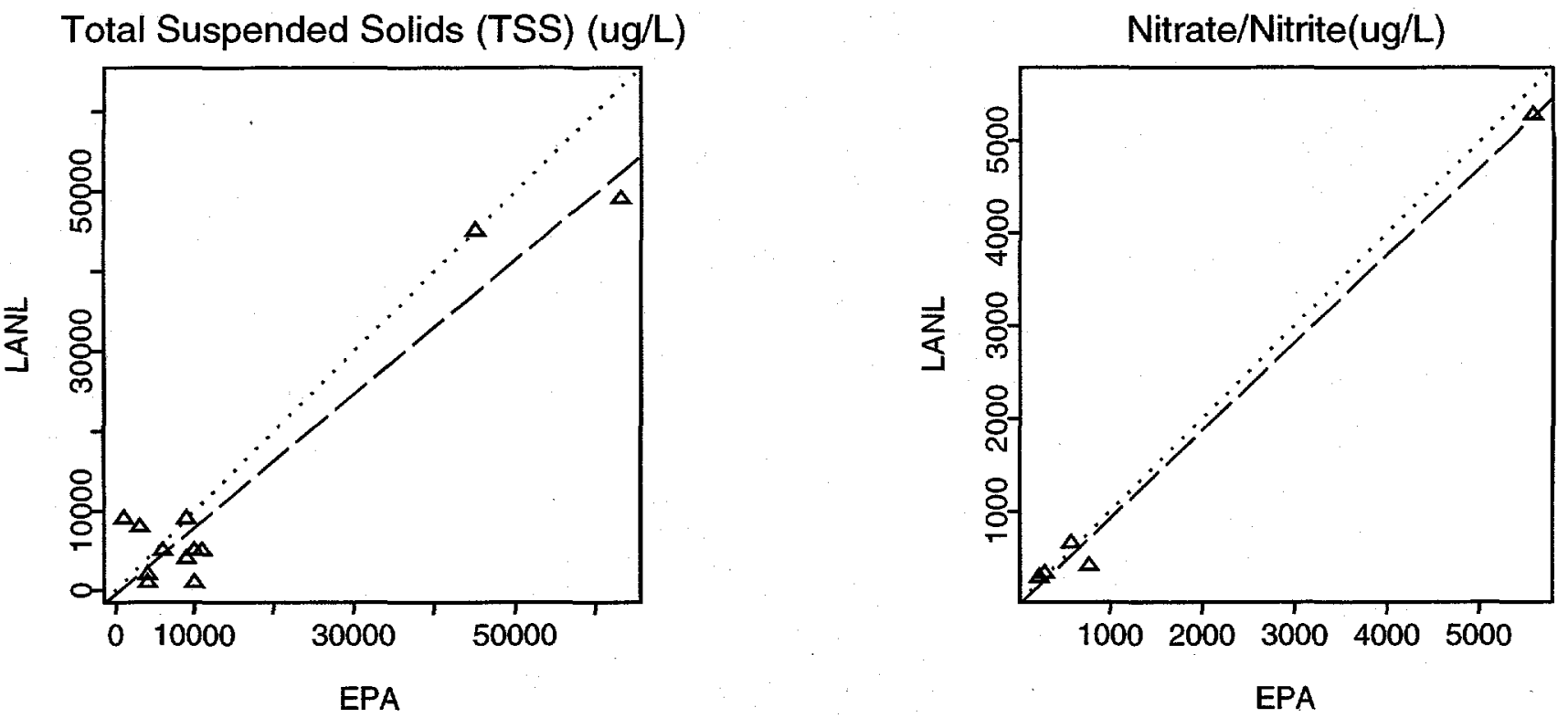

..... Reference Line

.-.- Filtered regression

- Unfiltered regression Missing line = no regression relationship
- $\quad$ Filtered samples

$\Delta \quad$ Unfiltered samples 
Inorganics: NMED vs. EPA
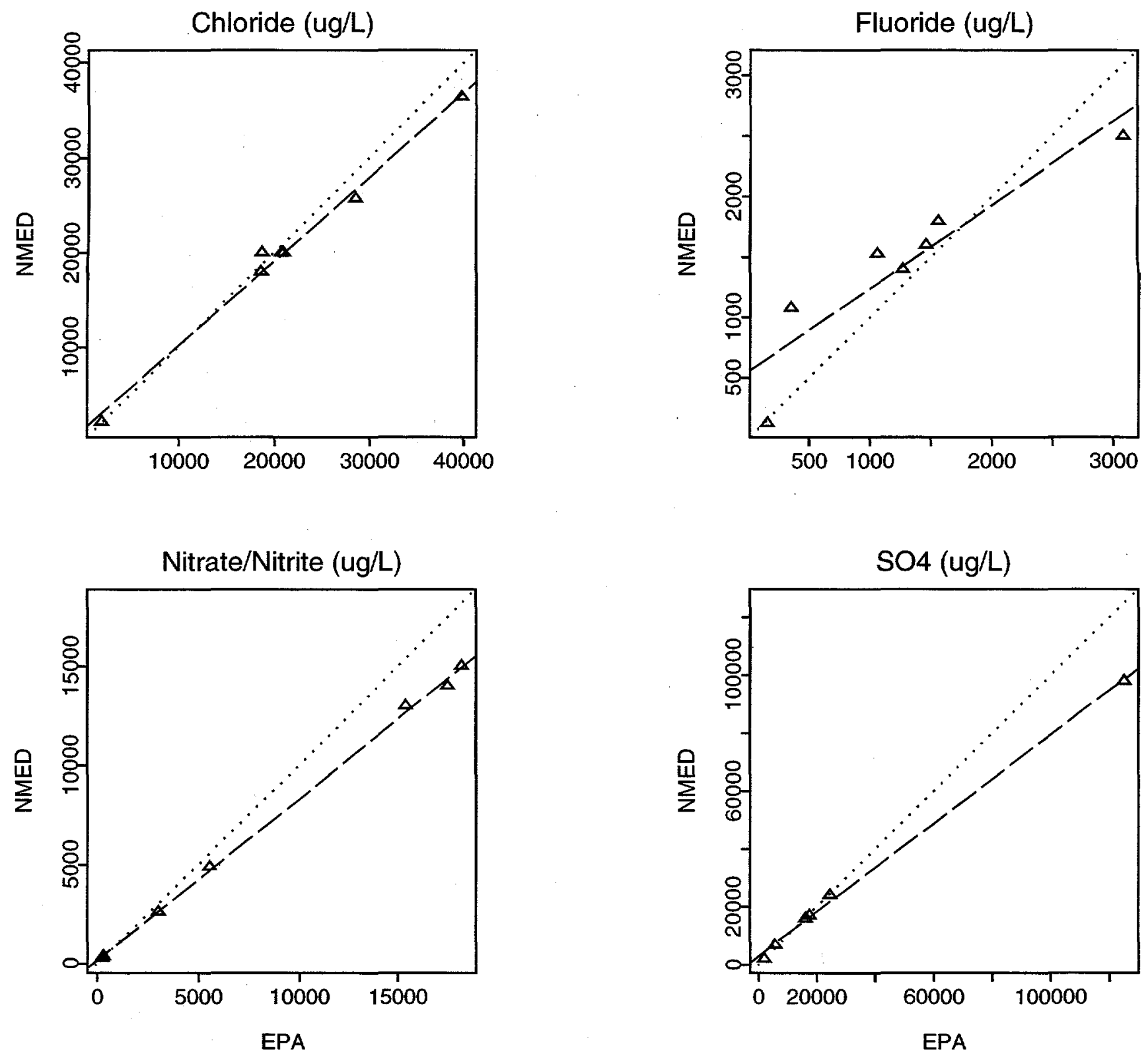

..... Reference Line

Filtered regression

- $\quad$ Filtered samples

- Unfiltered regression

$\Delta \quad$ Unfiltered samples Missing line $=$ no regression relationship 
Inorganics: NMED vs. EPA

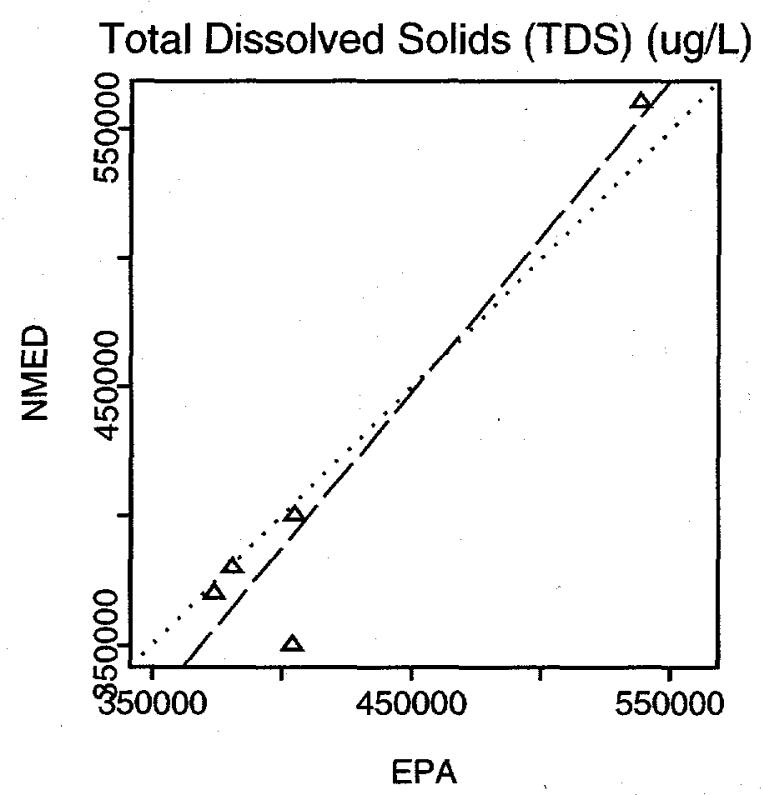

..... Reference Line

-... Filtered regression

- Filtered samples

- Unfiltered regression

$\Delta \quad$ Unfiltered samples Missing line = no regression relationship 
Radionuclides: LANL vs. EPA
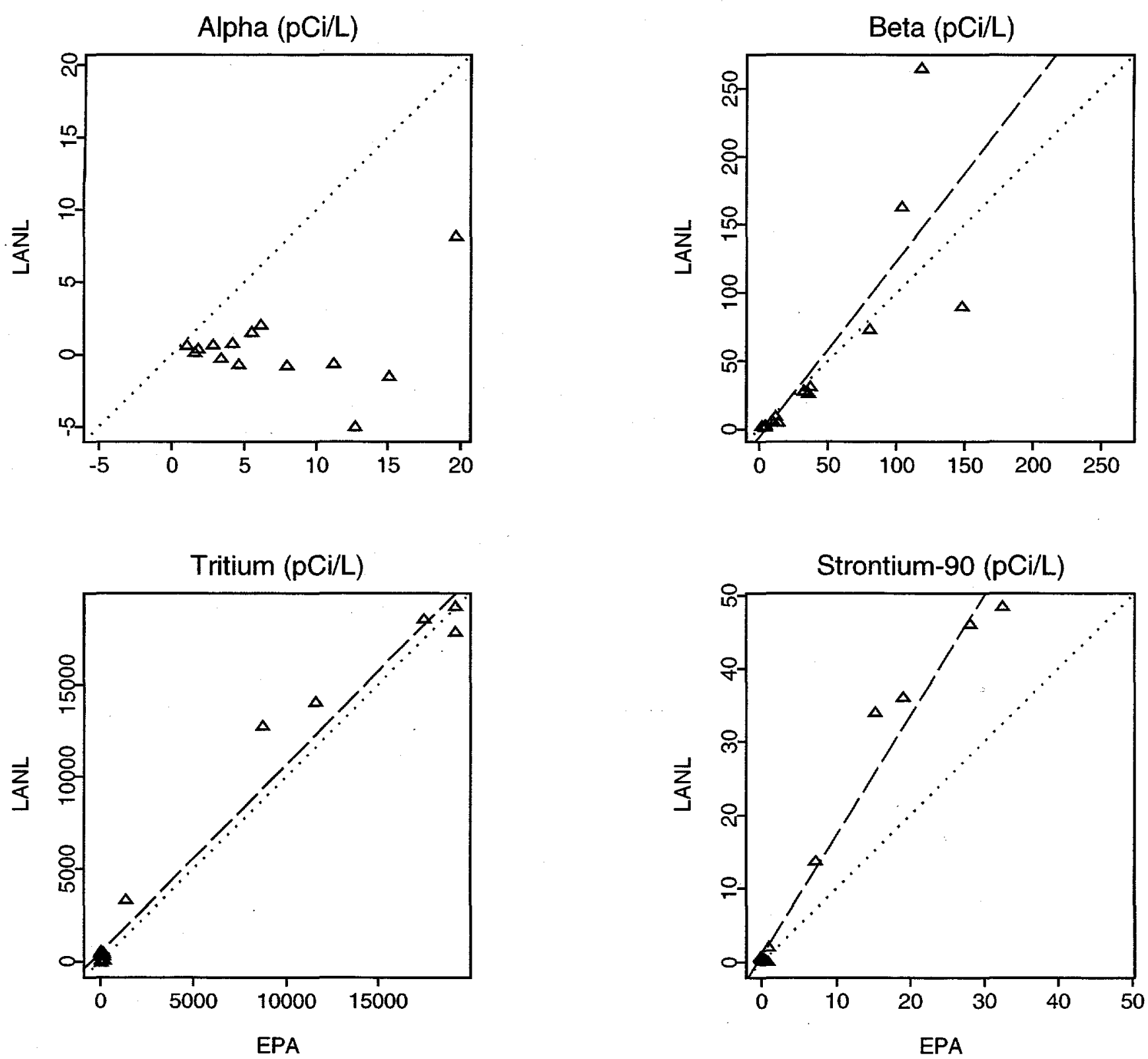

..... Reference Line

-..- Filtered regression

- $\quad$ Filtered samples

- Unfiltered regression

$\Delta \quad$ Unfiltered samples Missing line = no regression relationship 
Radionuclides: NMED vs. EPA
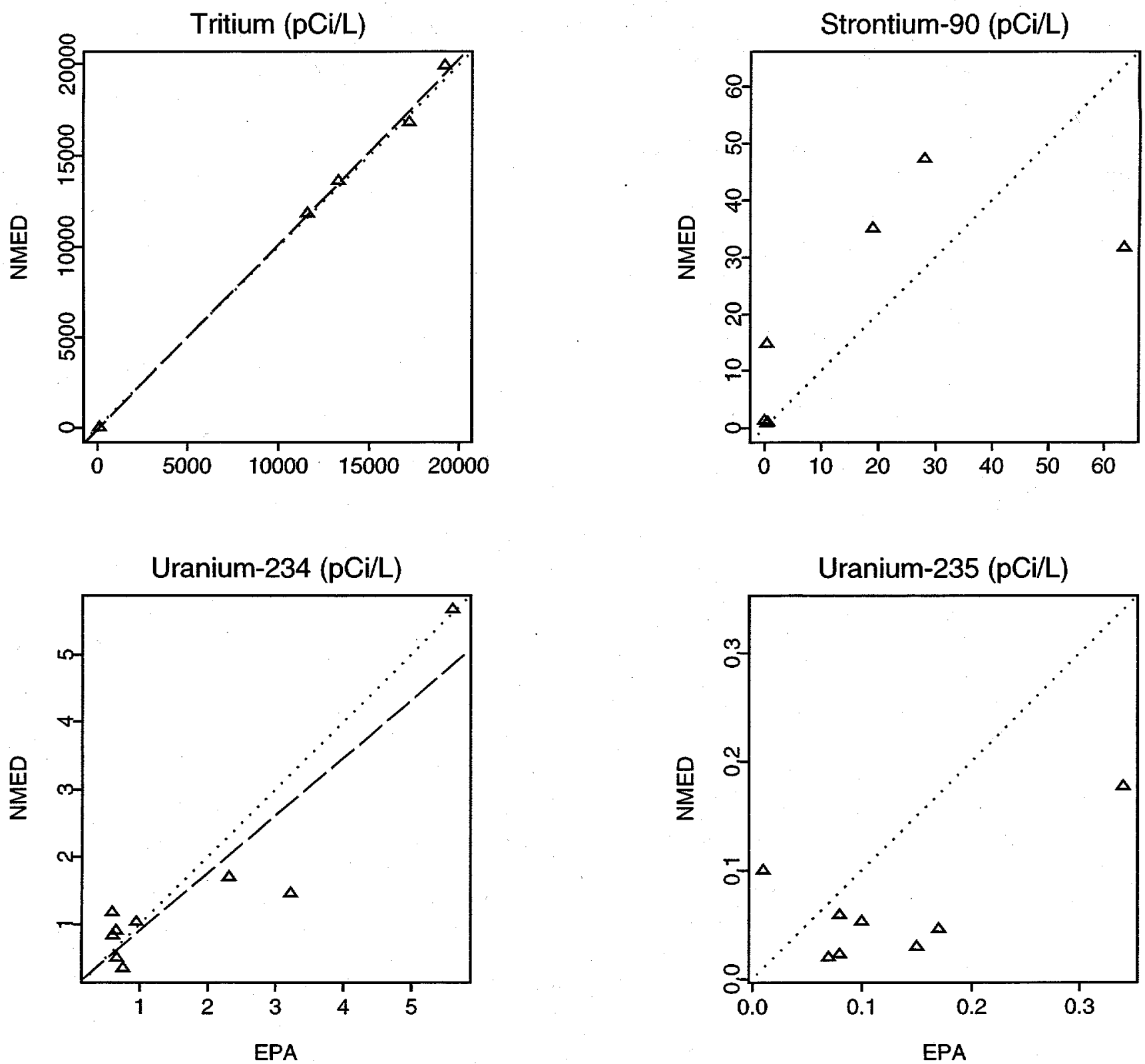

..... Reference Line

-.-. Filtered regression

- Unfiltered regression Missing line = 
Radionuclides: NMED vs. EPA

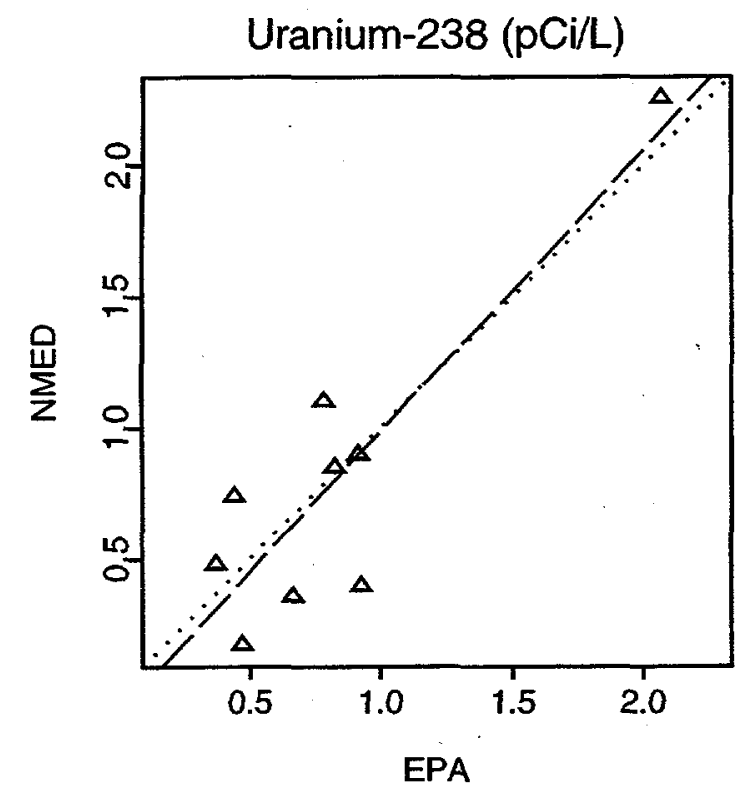

..... Reference Line

-.-.- Filtered regression Unfiltered regression

- Filtered samples

$\Delta \quad$ Unfiltered samples Missing line = no regression relationship 


\section{APPENDIX E GROUNDWATER QUALITY STANDARDS AND GUIDELINES}

Groundwater quality standards and guidelines set by the U.S. Department of Energy (DOE) and the State of New Mexico are included in this table for comparison and reference. DOE guidelines included for reference are the DOE Derived Concentration Guides for drinking water systems and for water ingestion in uncontrolled areas. The drinking water guidelines are included for comparison, as these guidelines are only directly applicable to the public water supply and not to monitoring wells. The New Mexico Environmental Improvement Board Maximum Contaminant Levels (MCLs) for inorganic chemicals and radioactivity in drinking water systems are provided. Other New Mexico standards included for reference and comparison are the New Mexico Water Quality Control Commission standards for groundwater of less than $10,000 \mathrm{mg} / \mathrm{L}$ total dissolved solids concentration or less; and the New Mexico Standards for Interstate and Intrastate Streams for Livestock Watering Limits 


\begin{tabular}{|c|c|c|c|c|c|c|c|c|c|}
\hline Suite & Analyie & UNITS & DOE DCG & $\begin{array}{l}\text { OE DW } \\
\text { DCG }\end{array}$ & $\begin{array}{c}\text { NM } \\
\text { Primary } \\
\text { DW } \\
\end{array}$ & $\begin{array}{c}\text { NM } \\
\text { Sec/HA/ } \\
\text { Act DW } \\
\end{array}$ & $\begin{array}{c}\text { NM } \\
\text { Livestock }\end{array}$ & $\begin{array}{l}\text { NM } \\
\text { GW }\end{array}$ & Min Std \\
\hline $\operatorname{Rad}$ & Am-24l & $\mathrm{PCl} / \mathrm{L}$ & 30 & 1.2 & & & & & 1.2 \\
\hline Rad & Cs- 137 & $\mathrm{PCl} / \mathrm{L}$ & 3000 & 120 & & & & & 120 \\
\hline Rad & ALPHA & $\mathrm{PCl} / \mathrm{L}$ & 30 & & Is & & 15 & & 15 \\
\hline Rad & BETA & $\mathrm{PCl} / \mathrm{L}$ & 1000 & & & 50 & & & 50 \\
\hline Rad & GAMMA & $\mathrm{PCl} / \mathrm{L}$ & & & & & & & \\
\hline Rad & $\mathrm{H}-3$ & $\mathrm{PCI} / \mathrm{L}$ & 2000000 & 80000 & 20000 & & 20000 & & 20000 \\
\hline Rad & Pu-238 & $\mathrm{PCI} / \mathrm{L}$ & 40 & 1.6 & & & & & 1.6 \\
\hline Rad & Pu-239.240 & $\mathrm{PCl} / \mathrm{L}$ & 30 & 1.2 & & & & & 1.2 \\
\hline Rad & Sr-90 & $\mathrm{PCI} / \mathrm{L}$ & 1000 & 40 & $\varepsilon$ & & 8 & & \\
\hline Rad & u & UG/L & 800 & 30 & 20 & & & 5000 & 20 \\
\hline Metals & $\mathrm{Ag}$ & UG/L & & & & & & 50 & 50 \\
\hline Metals & $\mid \mathrm{Al}$ & UG/L & & & & 200 & 5000 & 5000 & 200 \\
\hline Metals & As & $U G / L$ & & & 50 & & 200 & 100 & 50 \\
\hline Metals & $B$ & UG $/ L$ & & & & & 5000 & 750 & 750 \\
\hline Metals & $\mathrm{Ba}$ & UG $/ \mathrm{L}$ & & & 2000 & & & 1000 & 1000 \\
\hline Metals & $\mathrm{Be}$ & UG/L & & & $z$ & & & & 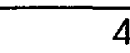 \\
\hline Metals & Cd & $U G / L$ & & & 5 & & 50 & 10 & \\
\hline Metals & Co & $U \in / L$ & & & & & 1000 & 50 & 50 \\
\hline Metals & $\mathrm{Cr}$ & UUG $/ \mathrm{L}$ & & & 100 & & 1000 & 50 & 50 \\
\hline Metals & $\mathrm{Cu}$ & $U G / L$ & & & & 1300 & 500 & 1000 & 500 \\
\hline Metals & $\mathrm{Fe}$ & $U G / L$ & & & & 300 & & 1000 & 300 \\
\hline Metals & $\mathrm{Hg}$ & UG/L & & & 2 & 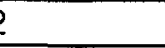 & 10 & 2 & \\
\hline Metals & $M n$ & UG $/ \mathrm{L}$ & & & & 50 & & 200 & 50 \\
\hline Metals & Mo & UG/L & & & & & & 1000 & 1000 \\
\hline Metals & $\mathrm{Ni}$ & UG/L & & & 100 & & & 200 & 100 \\
\hline Metals & $\mathrm{Pb}$ & $U \in / L$ & & & & 15 & 100 & 50 & 15 \\
\hline Metals & $\mathrm{Sb}$ & UG $/ \mathrm{L}$ & & & 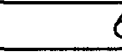 & & & & \\
\hline Metals & $\mathrm{Se}$ & $U G / L$ & & & $\overline{5}$ & & 2 & 50 & \\
\hline Metals & Sr & UG/L & & & & 25000 & & & 25000 \\
\hline Metals & $\pi$ & $U G / L$ & & & 2 & $?$ & & & 20 \\
\hline Metals & V & UG/L & & & & 80 & 100 & & 80 \\
\hline Metals & $\mathrm{Zn}$ & UG $/ \mathrm{L}$ & & & & 5000 & 25000 & 10000 & 5000 \\
\hline General Inorg. & $\mathrm{Cl}$ & $M G / L$ & & & & 250 & & 250 & 250 \\
\hline General Inorg. & $\mathrm{CN}$ & $M G / L$ & & & 0.2 & & & 0.2 & 0.2 \\
\hline General Inorg. & $F$ & $M G / L$ & & & 2 & 4 & & 1.6 & 1.6 \\
\hline General Inorg. & $\mathrm{Na}$ & $M G / L$ & & & & 20 & & & 20 \\
\hline General Inorg. & NO3-N & $M G / L$ & & & 10 & & & 10 & 10 \\
\hline General Inorg. & $\mathrm{PH}$ & & & & & 8.5 & & 9 & 8.5 \\
\hline General Inorg. & 504 & $M G / L$ & & & 500 & 250 & & .600 & 250 \\
\hline General Inorg. & TDS & $\mathrm{MG} / \mathrm{L}$ & & & & 500 & & 1000 & 500 \\
\hline
\end{tabular}

* EPA proposed drinking water standard for uranium. 
DOE DCG (Derived Concentration Guidelines for water ingestion in uncontrolled areas): US Department of Energy, "Radiation Protection of the Public and the Environment," US Department of Energy Order 5400.5 (February 1990).

DOE DW DCG (Derived Concentration Guidelines for drinking water systems): US Department of Energy, "Radiation Protection of the Public and the Environment," US Department of Energy Order 5400.5 (February 1990).

NM Primary DW (Primary Drinking Water Standards adopted by NMEIB and equivalent to EPA MCLs ): New Mexico Environmental Improvement Board, State of New Mexico, "New Mexico Drinking Water Regulations" (as amended through January 1995).

NM Sec/HA/Act DW (Secondary/Health Advisory/Action Levels standards adopted by NMEIB and equivalent to EPA MCLs): New Mexico Environmental Improvement Board, State of New Mexico, "New Mexico Drinking Water Regulations" (as amended through January 1995).

NM Livestock (Stream standards to protect livestock watering): New Mexico Water Quality Control Commission, "Water Quality Standards for Interstate and Intrastate Streams in New Mexico," (as amended through January 25, 1995).

NM GW (New Mexico Ground Water Standards): New Mexico Water Quality Control Commission, "New Mexico.Water Quality Control Commission Regulations" (as amended through January 23, 1995). 


\section{APPENDIX F \\ COMPARISON OF DATA PAIRS AGAINST STANDARDS}

This appendix tabulates the proportion of data pairs that are in agreement on whether contaminant levels exceed regulatory standards. The table does not show the number of results exceeding a regulatory limit. Rather, the table shows the proportion of analytical results from Lab 1 that agrees with Lab 2's when compared against the regulatory limit (concordant results). If one result from the pair is above the regulatory standard and the second result is below, the pairs are not in agreement and are discordant. The proportion in agreement is:

Number of concordant pairs that are both above (or below) the regulatory limit/ Total number of data pairs for a specific analyte.

Data pairs for this comparison were selected from Appendix B. The minimum groundwater quality standards and guidelines used in this analysis are taken from Appendix E. Results from unfiltered and filtered waters were pooled.

\begin{tabular}{|cccc|}
\hline ANALYTE & NMED vs. EPA & NMED vs. LANL & LANL vs. EPA \\
\hline $\mathrm{Cl}$ & $6 / 6$ & $1 / 1$ & $5 / 5$ \\
$\mathrm{~F}$ & $5 / 6$ & $1 / 1$ & $5 / 5$ \\
NO3/NO2 & $6 / 6$ & $1 / 1$ & $5 / 5$ \\
$\mathrm{SO} 4$ & $6 / 6$ & $1 / 1$ & $5 / 5$ \\
$\mathrm{TDS}$ & $5 / 5$ &.-- & $3 / 5$ \\
$\mathrm{Al}$ & $10 / 10$ & $4 / 4$ & $19 / 26$ \\
$\mathrm{As}$ & $10 / 10$ & $4 / 4$ & $26 / 26$ \\
$\mathrm{~B}$ & $10 / 10$ & $4 / 4$ & $26 / 26$ \\
$\mathrm{Ba}$ & $10 / 10$ & $5 / 5$ & $26 / 26$ \\
$\mathrm{Be}$ & --- & --- & $26 / 26$ \\
$\mathrm{Cr}$ & $6 / 6$ & $3 / 3$ & $25 / 25$ \\
$\mathrm{Cu}$ & $7 / 7$ & $3 / 3$ & $26 / 26$ \\
$\mathrm{Fe}$ & $9 / 10$ & $5 / 5$ & $20 / 26$ \\
$\mathrm{Hg}$ & --- & -5 & $17 / 17$ \\
$\mathrm{Mn}$ & $10 / 10$ & $5 / 5$ & $24 / 26$ \\
$\mathrm{Mo}$ & $7 / 7$ & $2 / 2$ & $25 / 25$ \\
$\mathrm{~Pb}$ & $6 / 7$ & $3 / 3$ & $23 / 26$ \\
Am-241 &.-- & --- & $4 / 4$ \\
Gross alpha & $2 / 2$ & $1 / 1$ & $10 / 14$ \\
Gross beta & $2 / 2$ & $1 / 1$ & $14 / 14$ \\
$\mathrm{H}-3$ & $5 / 5$ & $3 / 3$ & $18 / 18$ \\
Pu-238 & $4 / 4$ & $4 / 4$ &.-- \\
Pu-239,240 & $4 / 4$ & $4 / 4$ &.-- \\
$\mathrm{Sr}-90$ & $9 / 10$ & $6 / 6$ & $13 / 14$ \\
\hline
\end{tabular}




\section{APPENDIX G EXPLORATORY GRAPHICAL ANALYSIS}

These exploratory graphical analyses depict the available data. The graphical analyses include box plots and histograms. These analyses are performed to provide a visual representation of the data, to determine the presence of outliers or other anomalous data that might affect statistical results and interpretations, and to gain a general understanding of the data. The plots allow a visual comparison between distributions of concentrations for results from pairs of laboratories. The differences of interest may include an overall shift in concentration (shift of central location), or when the centers are nearly equal, a difference in the tail of one distribution (numerous larger concentrations).

Histograms: The horizontal axis provides the concentrations in $\mathrm{pCi} / \mathrm{L}$ for radionuclides, or $\mu \mathrm{g} / \mathrm{L}$ for metals and general inorganic analytes, and the vertical axis provides the counts or number of observations in each concentration class. Concentration units are provided with each histogram, and the total number of observations included is also presented. An indication of the center of the distribution (the average value) is plotted as a dot. A vertical line represents the minimum regulatory standard or guideline for the analyte, taken from Appendix E.

Box Plots: Box plots can consist of a box, a line across the box, line brackets outside the box, and points outside the line brackets. The box area of the plot is the region between the $25^{\text {th }}$ percentile and the $75^{\text {th }}$ percentile of the data, or the interquartile range. The line in the box represents the median of the data. The line brackets give an interval of 1.5 times the interquartile range, outside of which data may be evaluated for their potential to be outliers.

Distributional Comparisons: Some care should be taken when interpreting these graphs with respect to the statistical analyses summarized in the main text and presented in earlier appendices. The statistical analyses performed evaluate the distribution of differences between pairs of results (e.g., paired t-tests to assess if the mean of the differences is zero), rather than evaluating the differences between distributions of each of two data sets (e.g., unpaired t-tests to assess if the means for two distributions are the same). The paired statistical analyses performed are warranted under an assumption that the data are reasonably paired. Although the distinction might appear subtle, direct comparison of the pairs of histograms or box plots can potentially be misleading. If differences are seen between the plots, then paired statistical analyses can be expected to provide confirmation. For example, uranium-235 results from NMED and EPA and gross alpha results from LANL and EPA appear to differ significantly. However, if a difference is not seen in a pair of plots, it is still reasonable for the corresponding paired difference tests to indicate a difference (because the paired data might not match the implicit ordering of the data in the plots). 


\section{Comparison of Chloride $(\mu \mathrm{g} / \mathrm{L})$ : NMED vs. EPA}
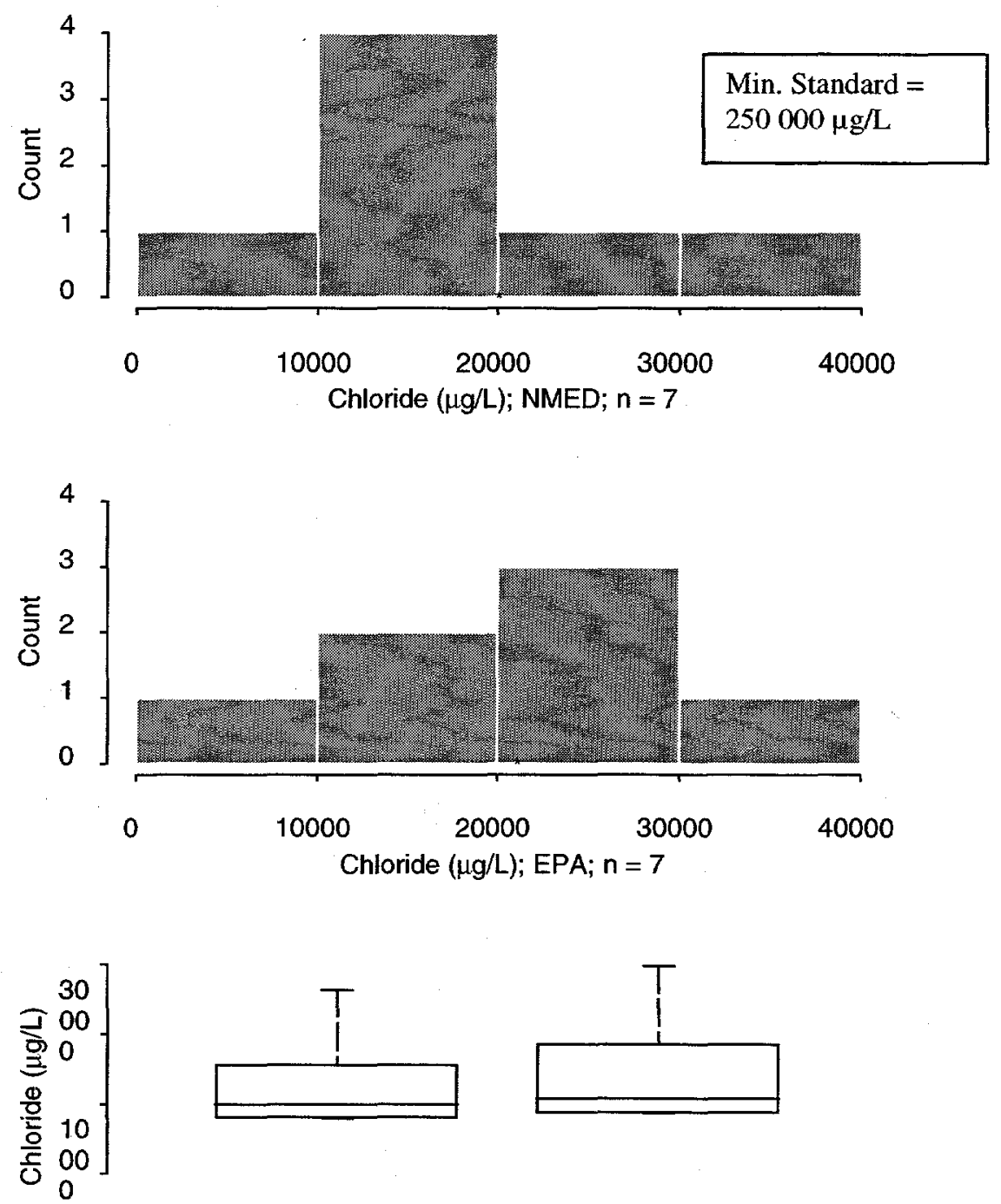

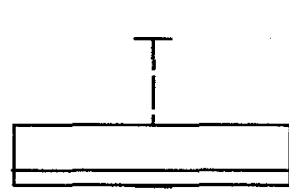

$\circ$

NMED

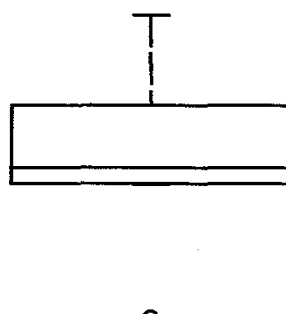

EPA 
Comparison of Chloride $(\mu \mathrm{g} / \mathrm{L})$ : NMED vs. EPA
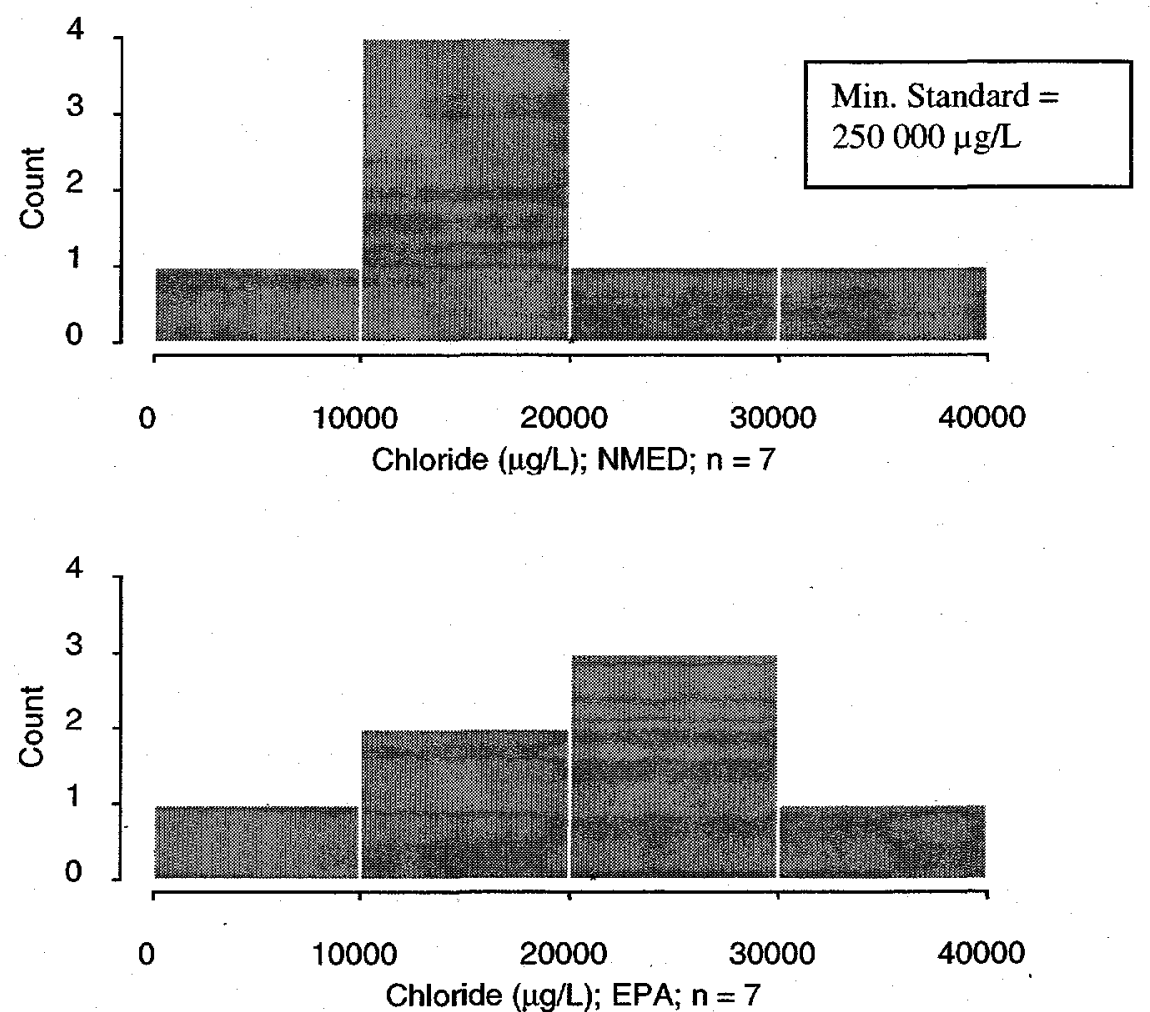

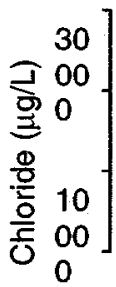

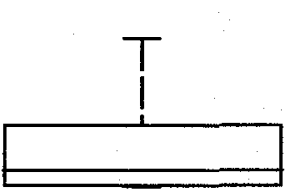

0

NMED

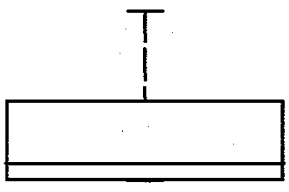

$\circ$

EPA 
Comparison of Fluoride $(\mu \mathrm{g} / \mathrm{L})$ : NMED vs. EPA
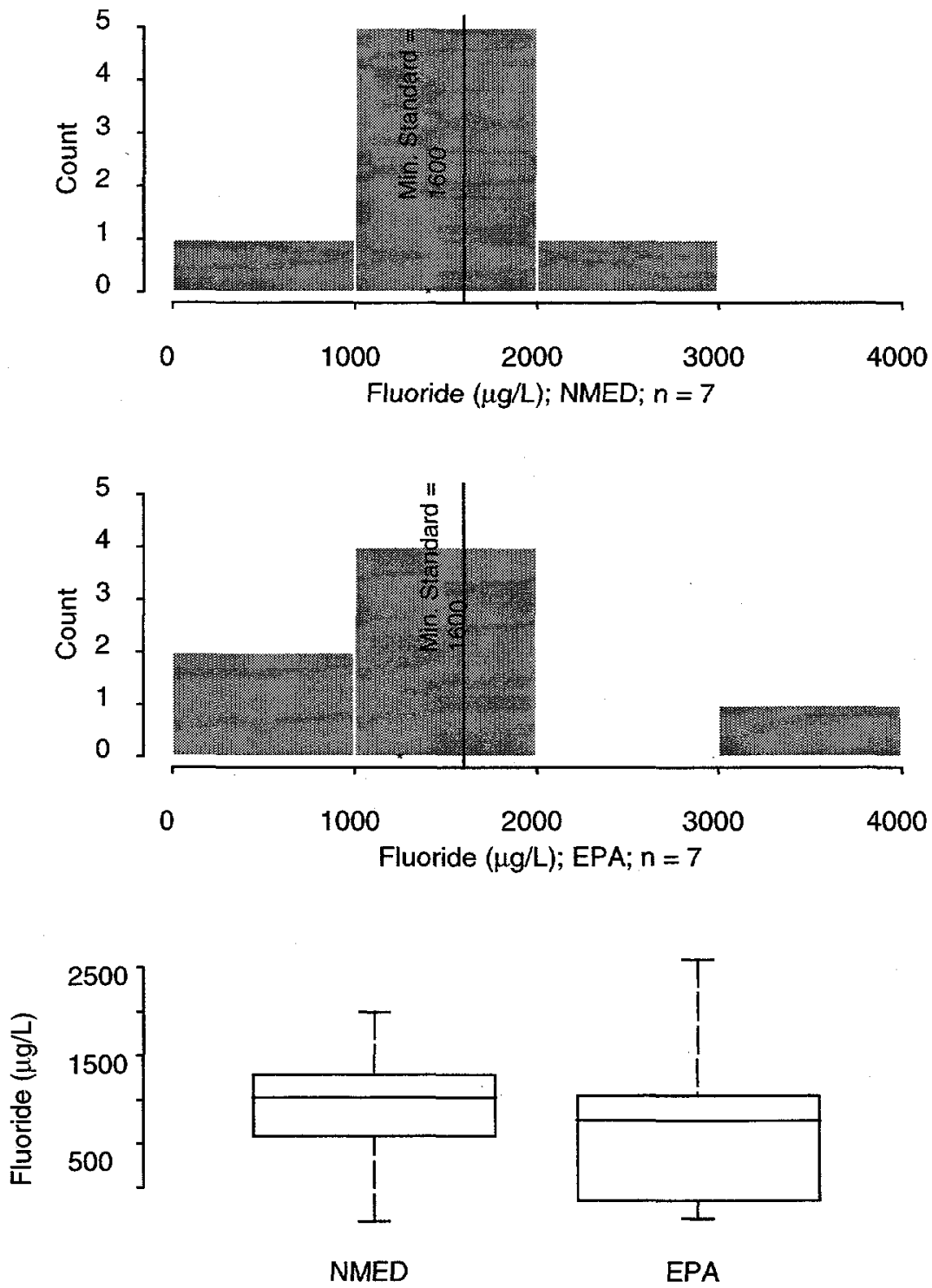


\section{Comparison of Nitrate/Nitrite-N ( $\mu \mathrm{g} / \mathrm{L})$ : NMED vs. EPA}
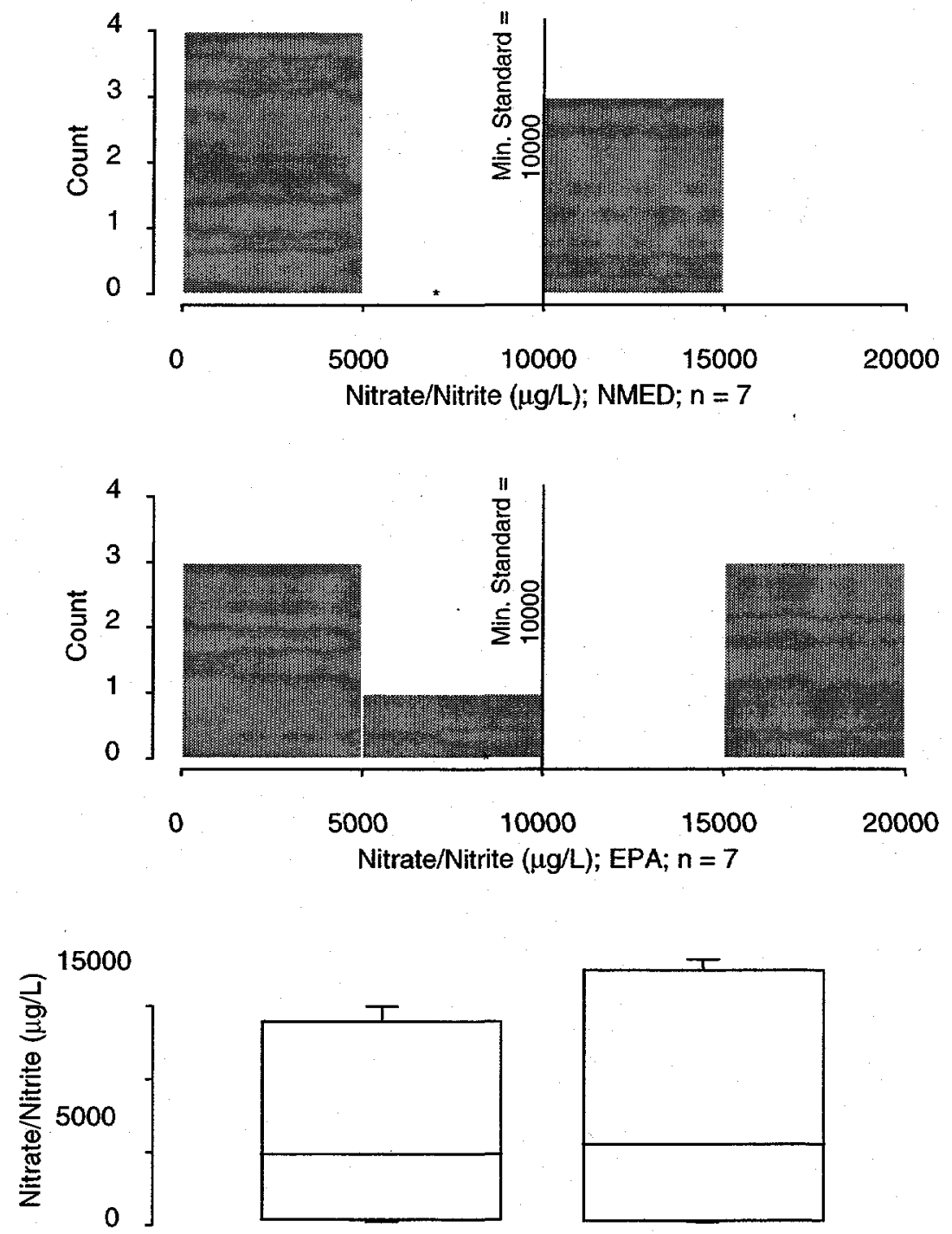

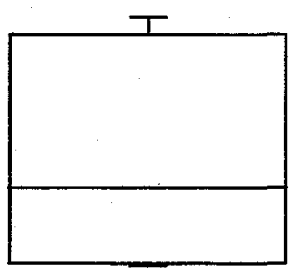

NMED

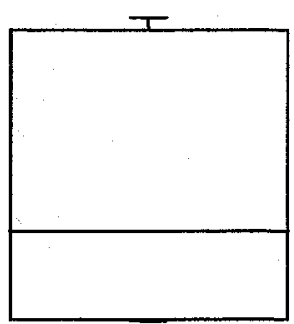

EPA 
Comparison of Sulfate $(\mu \mathrm{g} / \mathrm{L}) \quad$ : NMED vs. EPA
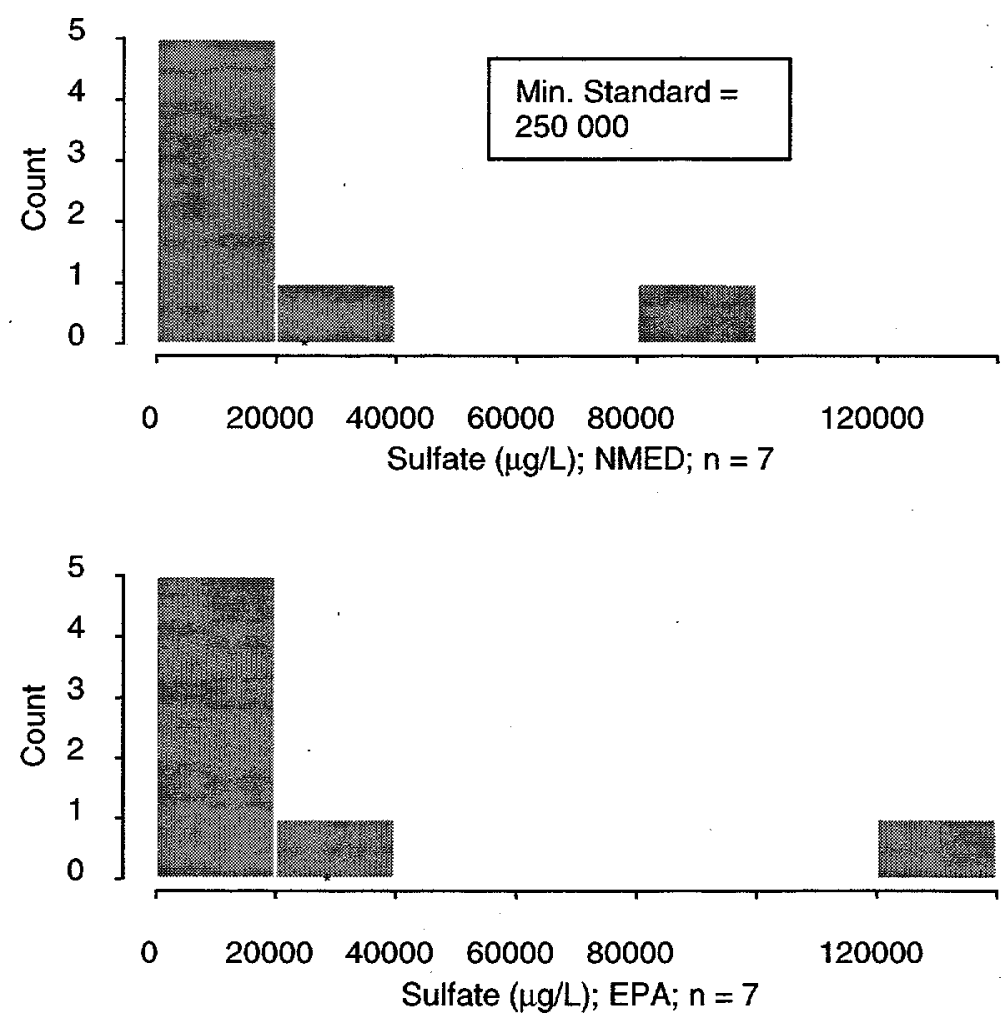

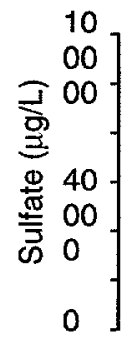

o

0
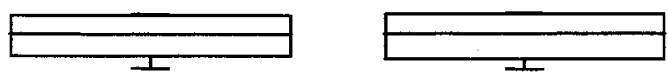

NMED

EPA 
Comparison of Total Dissolved Solids ( $\mu \mathrm{g} / \mathrm{L})$ : NMED vs. EPA
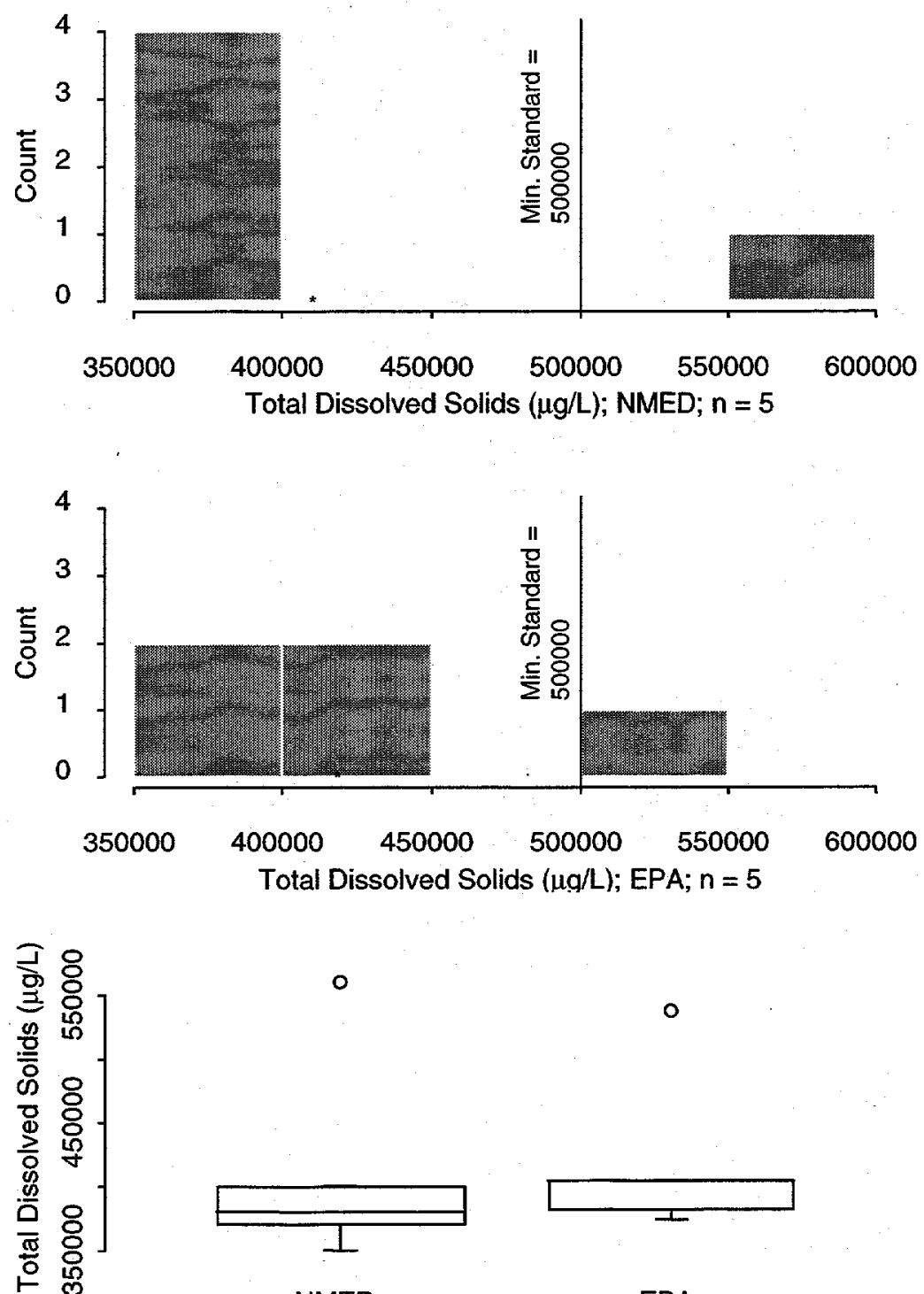

$\circ$

$\circ$
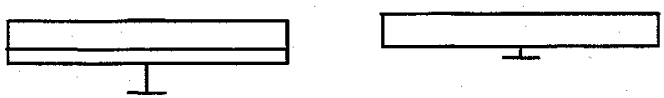

NMED

EPA 
Comparison of $\mathrm{Sr}-90$ (pCi/L): NMED vs. EPA
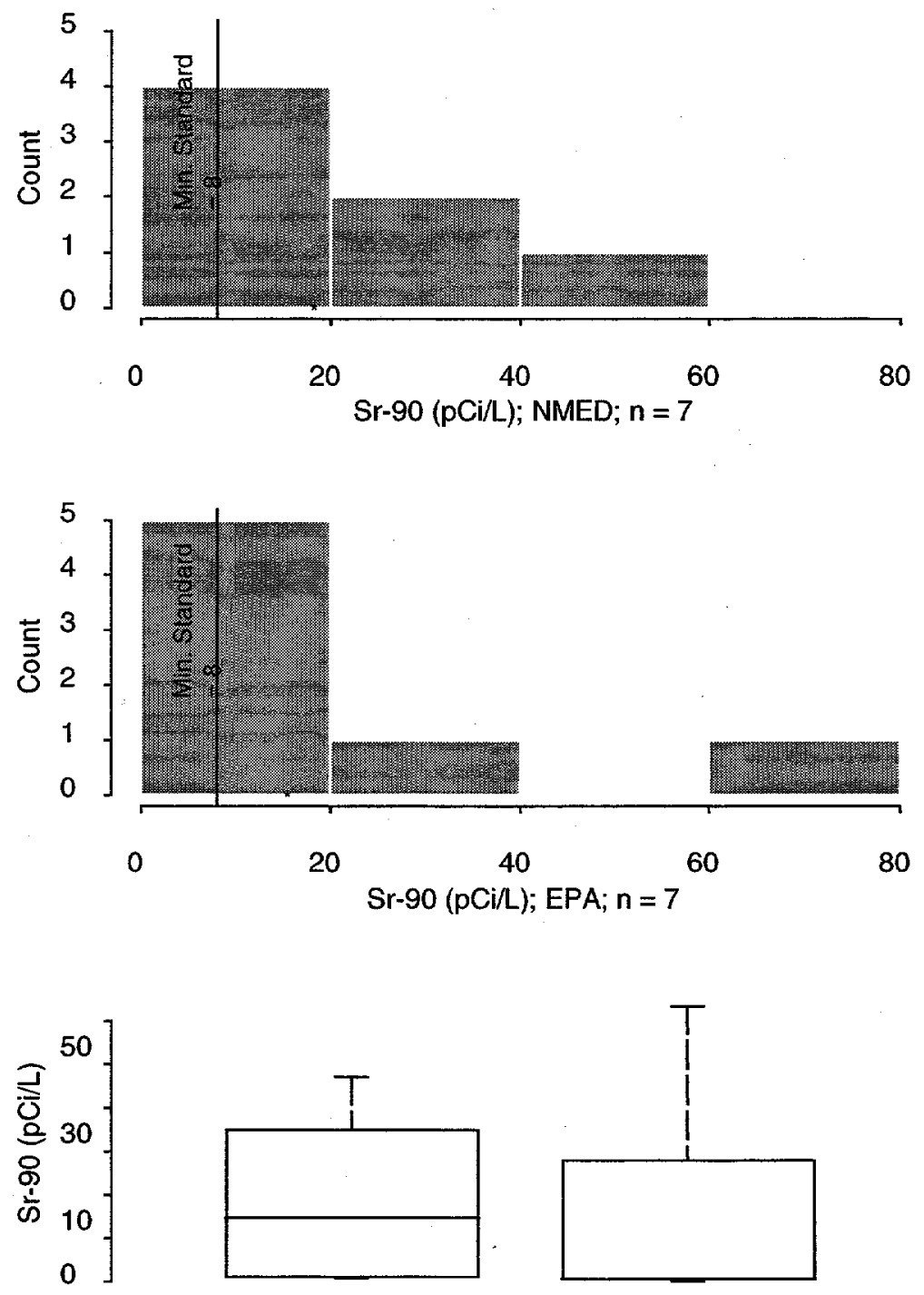

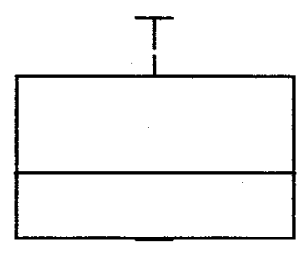

NMED

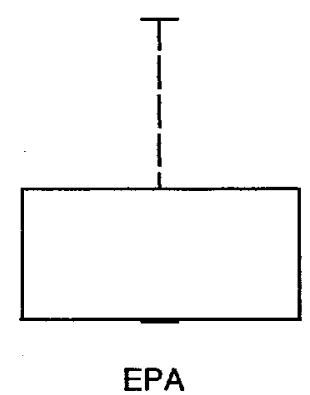


Comparison of U-234 (pCi/L) : NMED vs. EPA
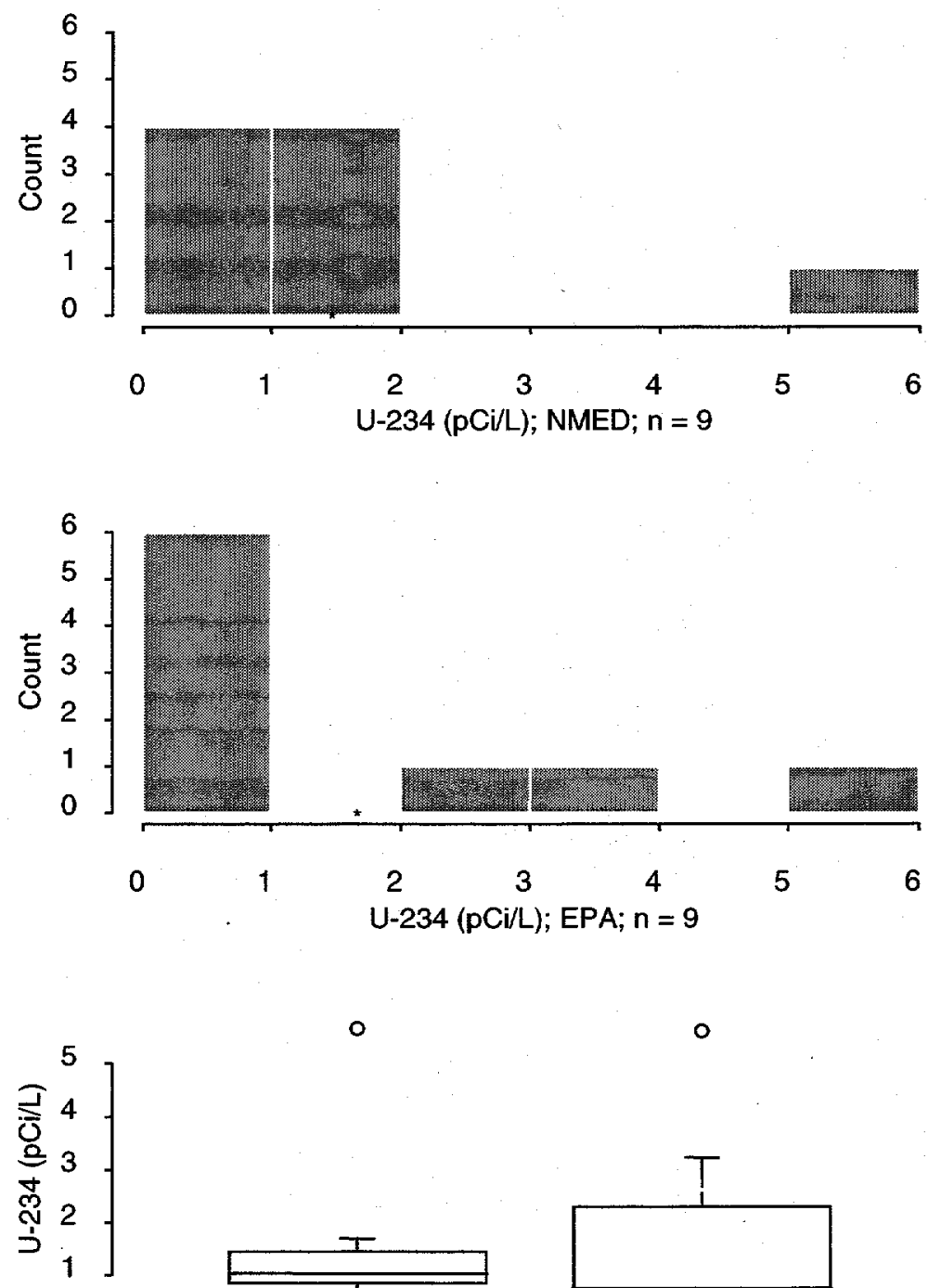

$\circ$

$\circ$

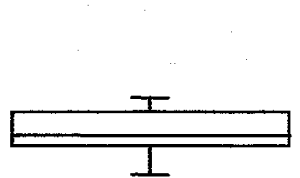

NMED

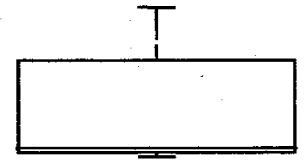

EPA 
Comparison of U-235 (pCi/L) : NMED vs. EPA
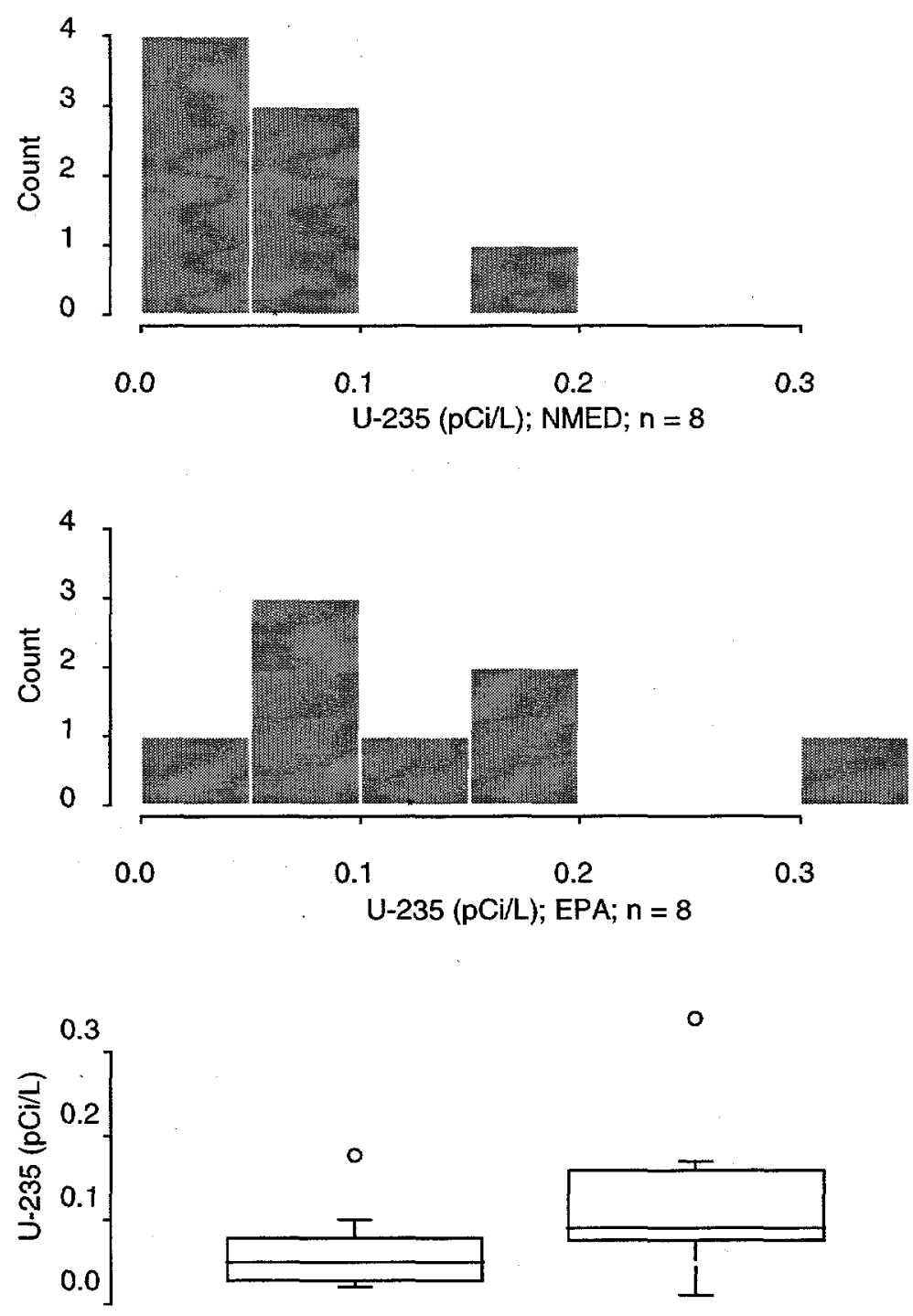

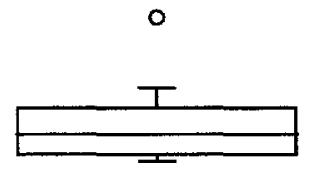

NMED $\circ$

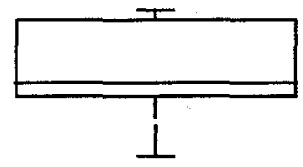

EPA 
Comparison of U-238 (pCi/L) : NMED vs. EPA
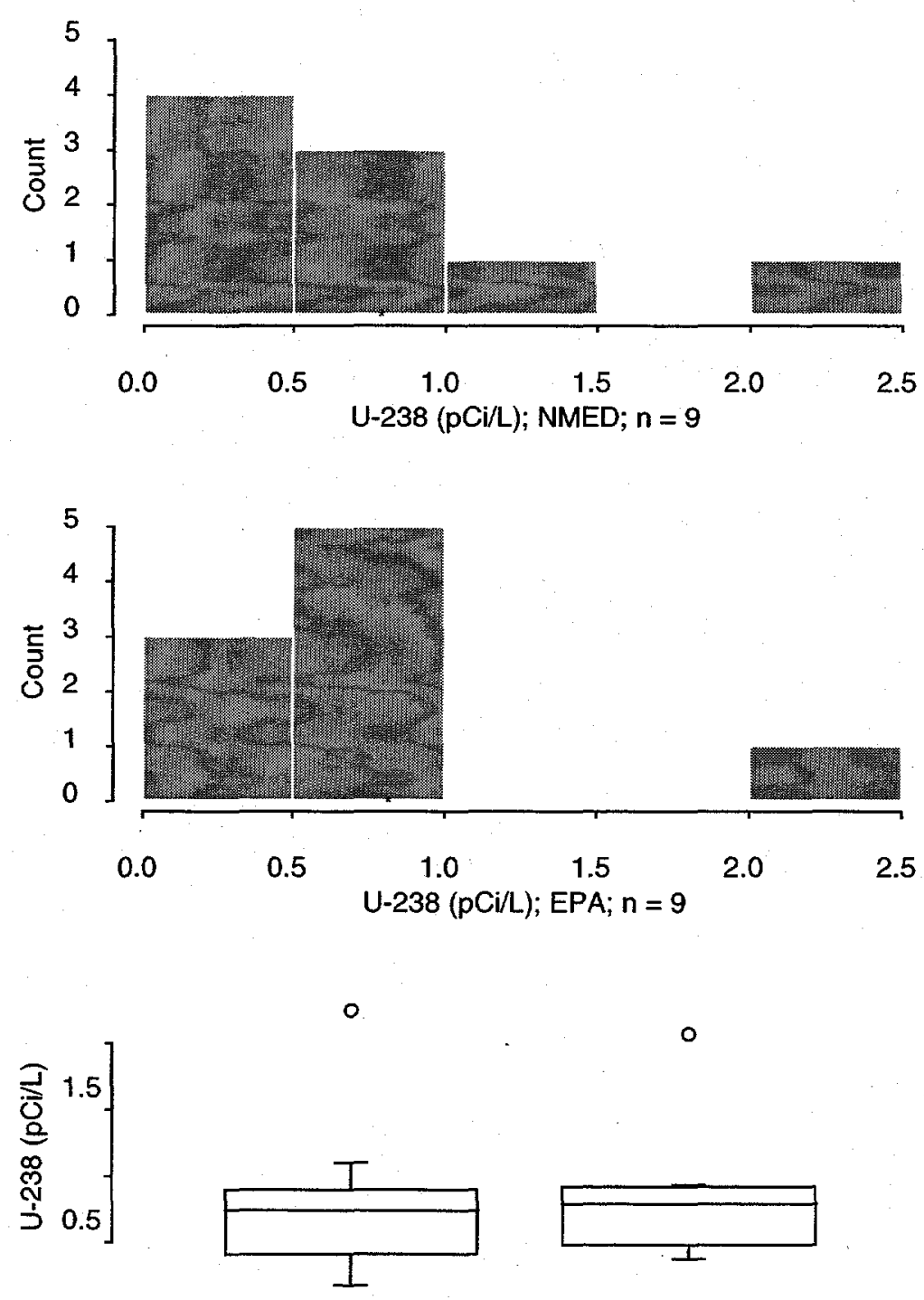

$\circ$

NMED

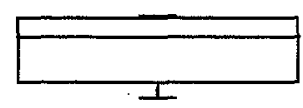

EPA 
Comparison of Alkalinity ( $\mu \mathrm{g} / \mathrm{L})$ : LANL vs. EPA
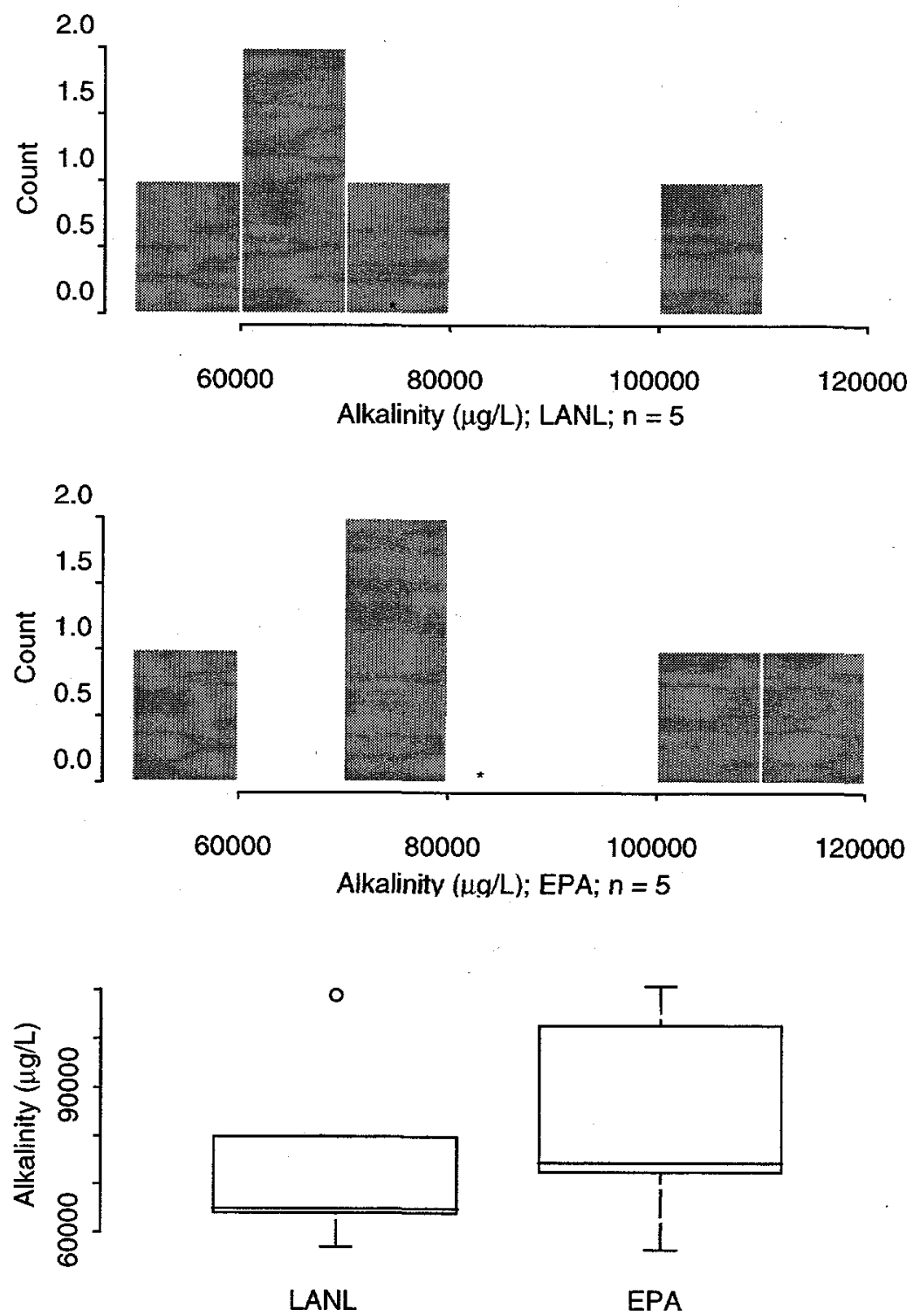
Comparison of Fluoride $(\mu \mathrm{g} / \mathrm{L})$ : LANL vs. EPA
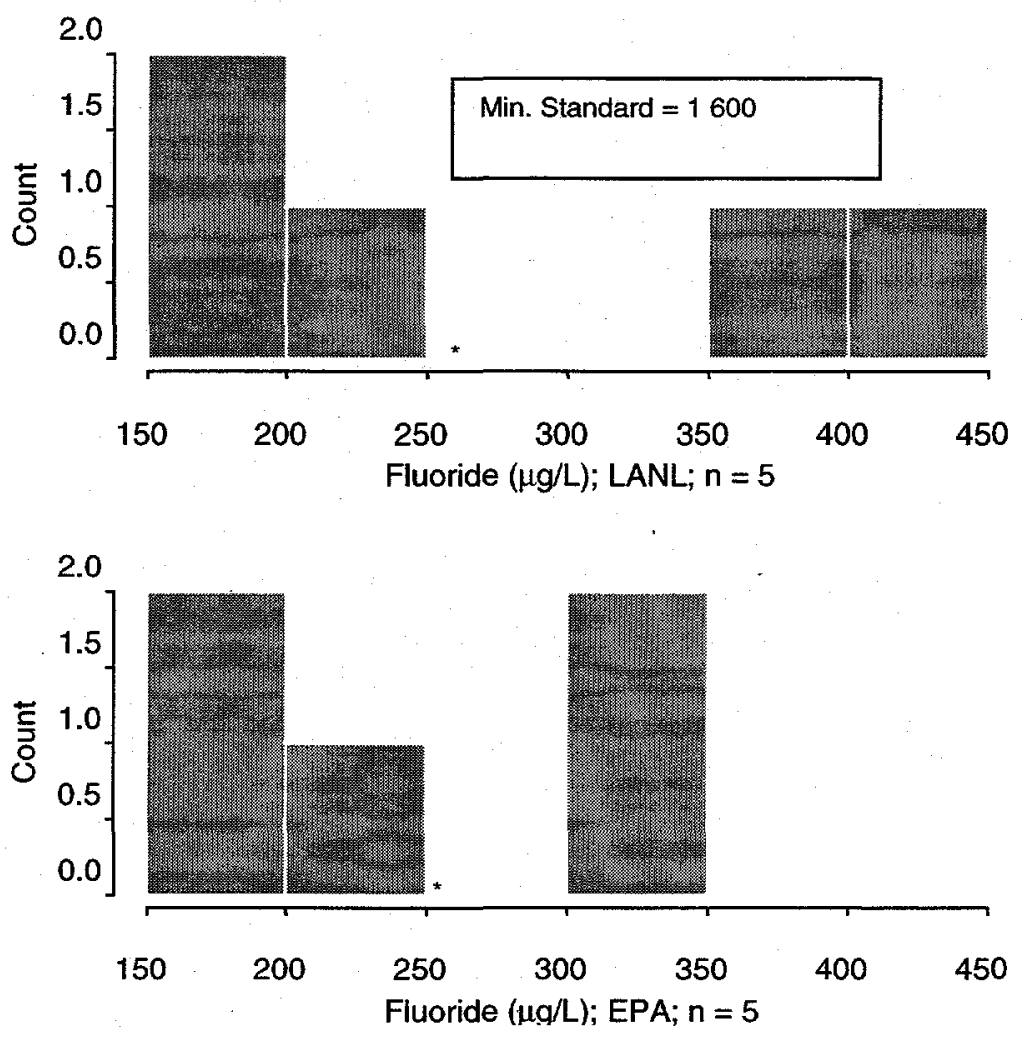

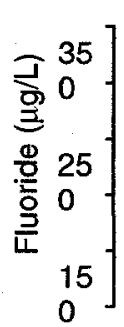

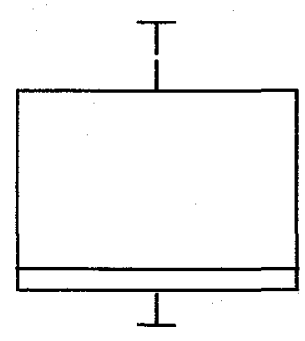

LANL

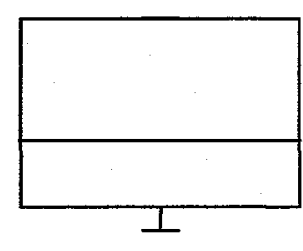

EPA 
Comparison of Chloride ( $\mu \mathrm{g} / \mathrm{L})$ : LANL vs. EPA
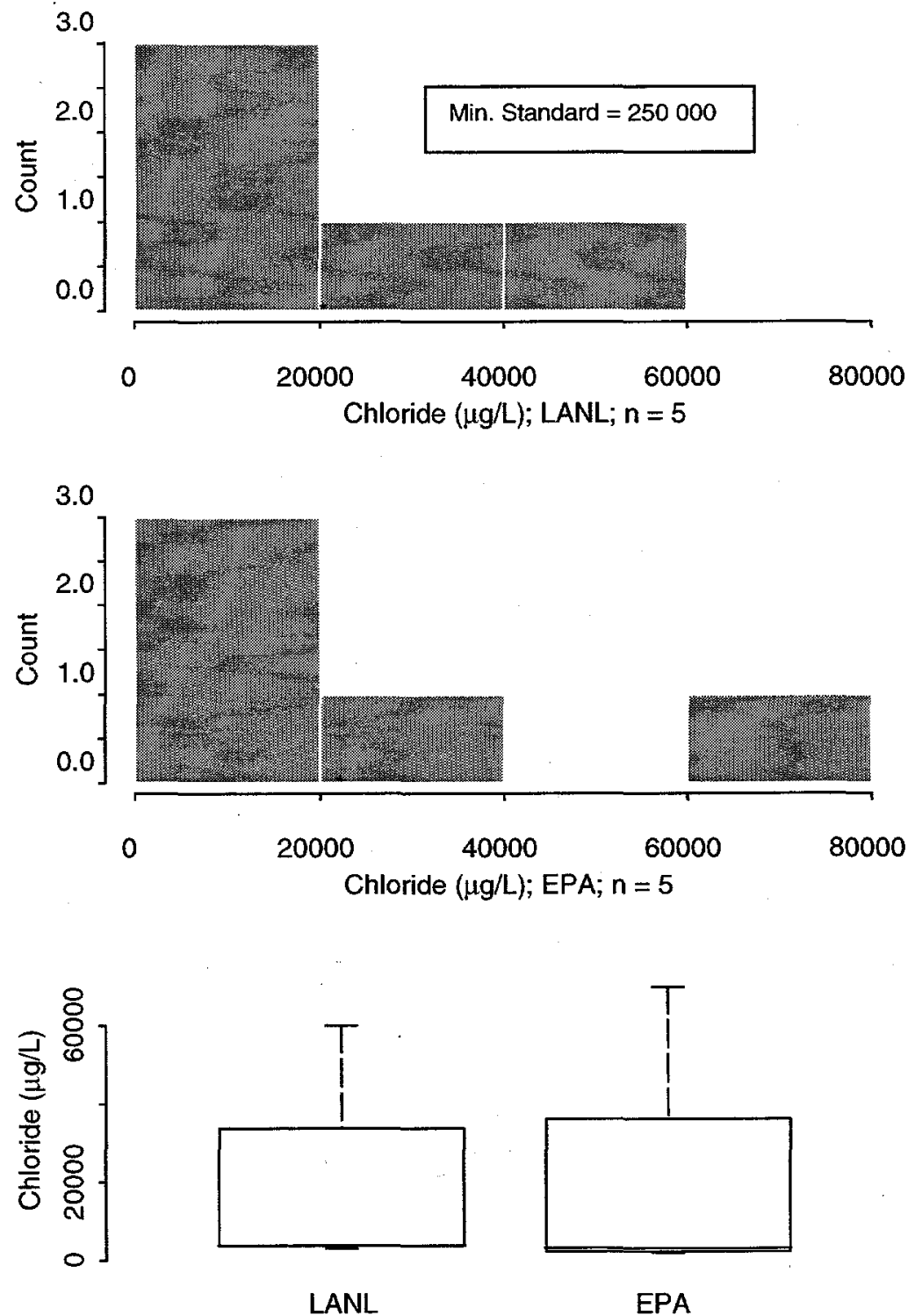
Comparison of Nitrate/Nitrite-N ( $\mu \mathrm{g} / \mathrm{L})$ : LANL vs. EPA
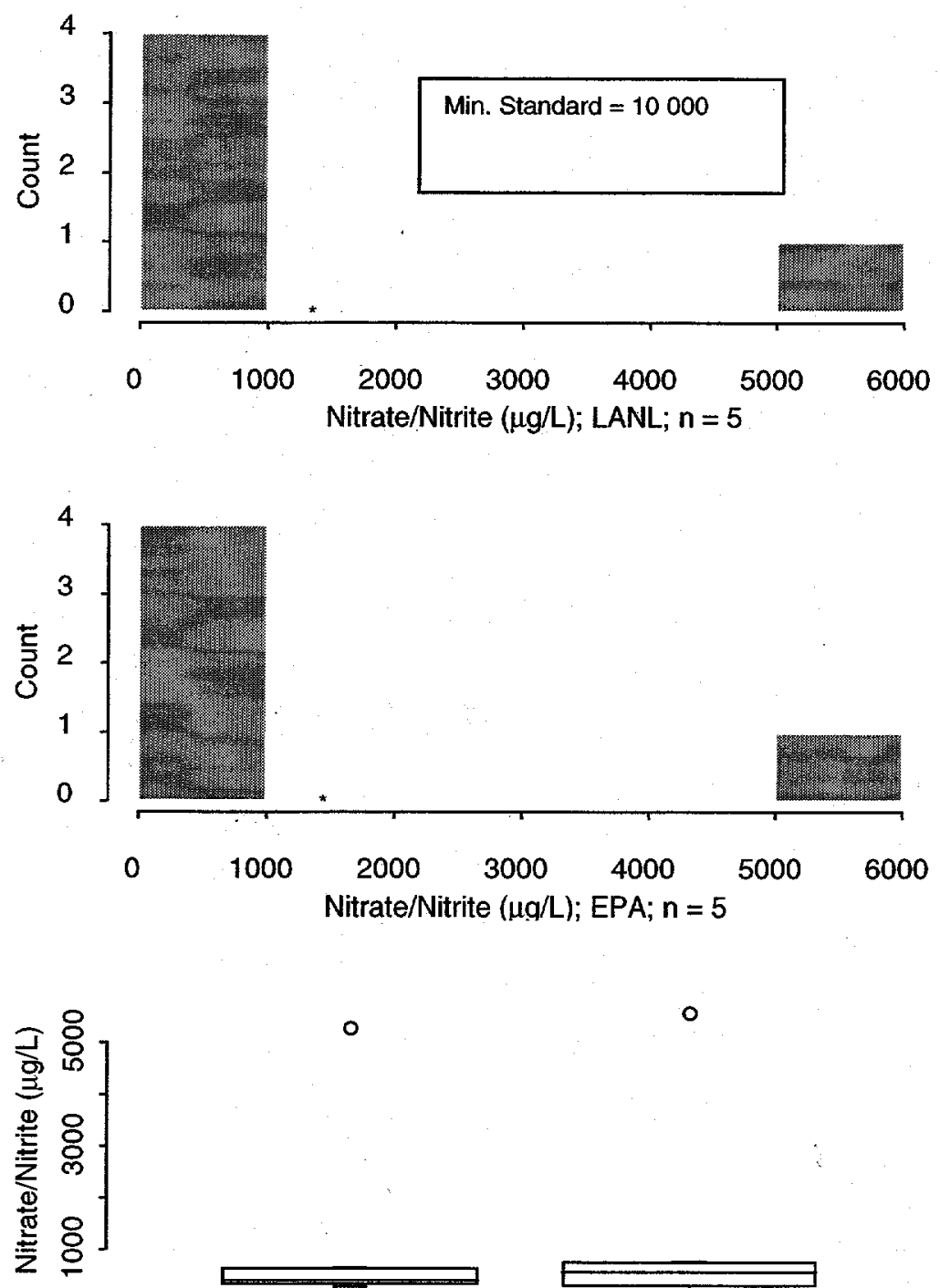

$\circ$

$\circ$

LANL

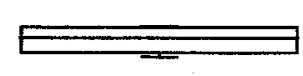

EPA 
Comparison of Sulfate $(\mu \mathrm{g} / \mathrm{L})$ : LANL vs. EPA
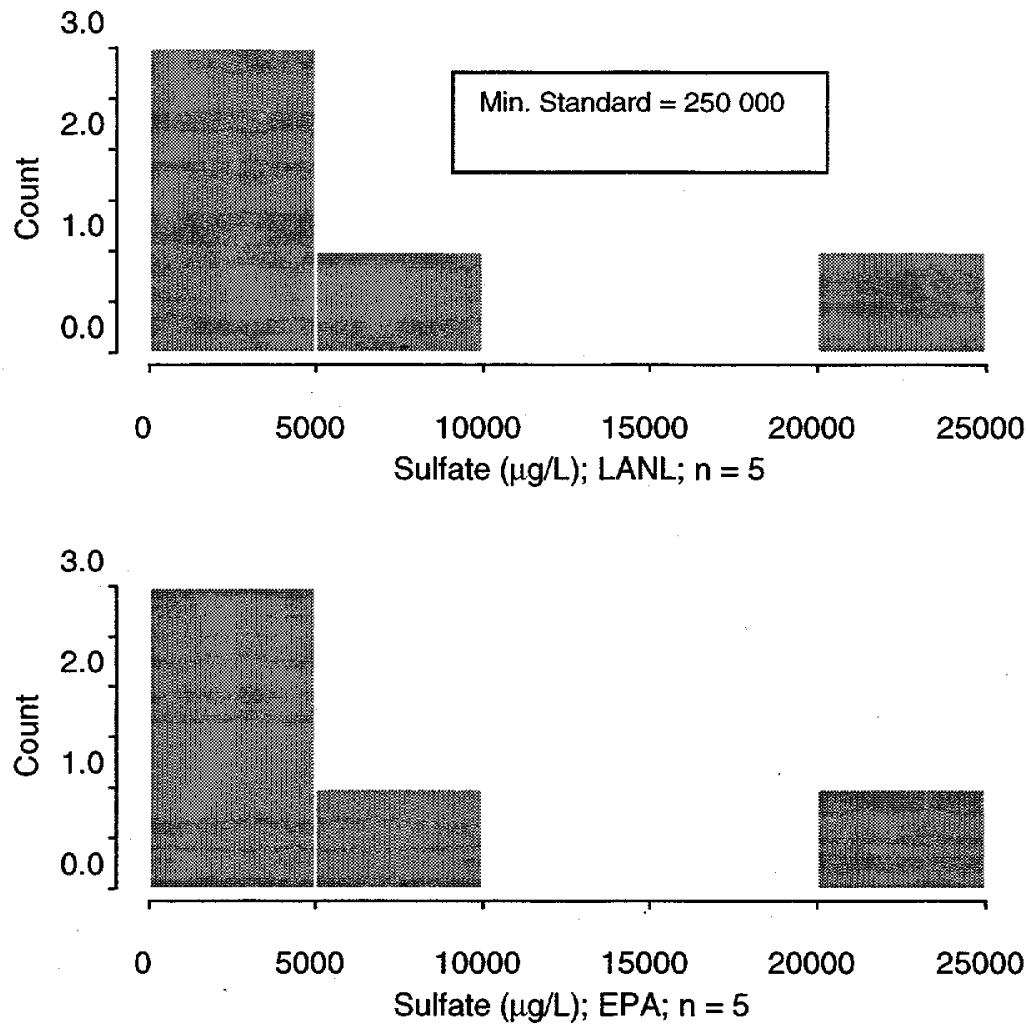

o

$\circ$

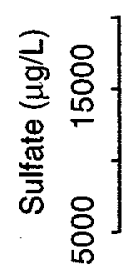

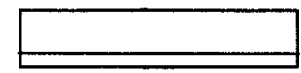

LANL

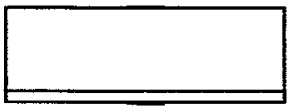

EPA 
Comparison of Total Dissolved Solids ( $\mu \mathrm{g} / \mathrm{L})$ : LANL vs. EPA
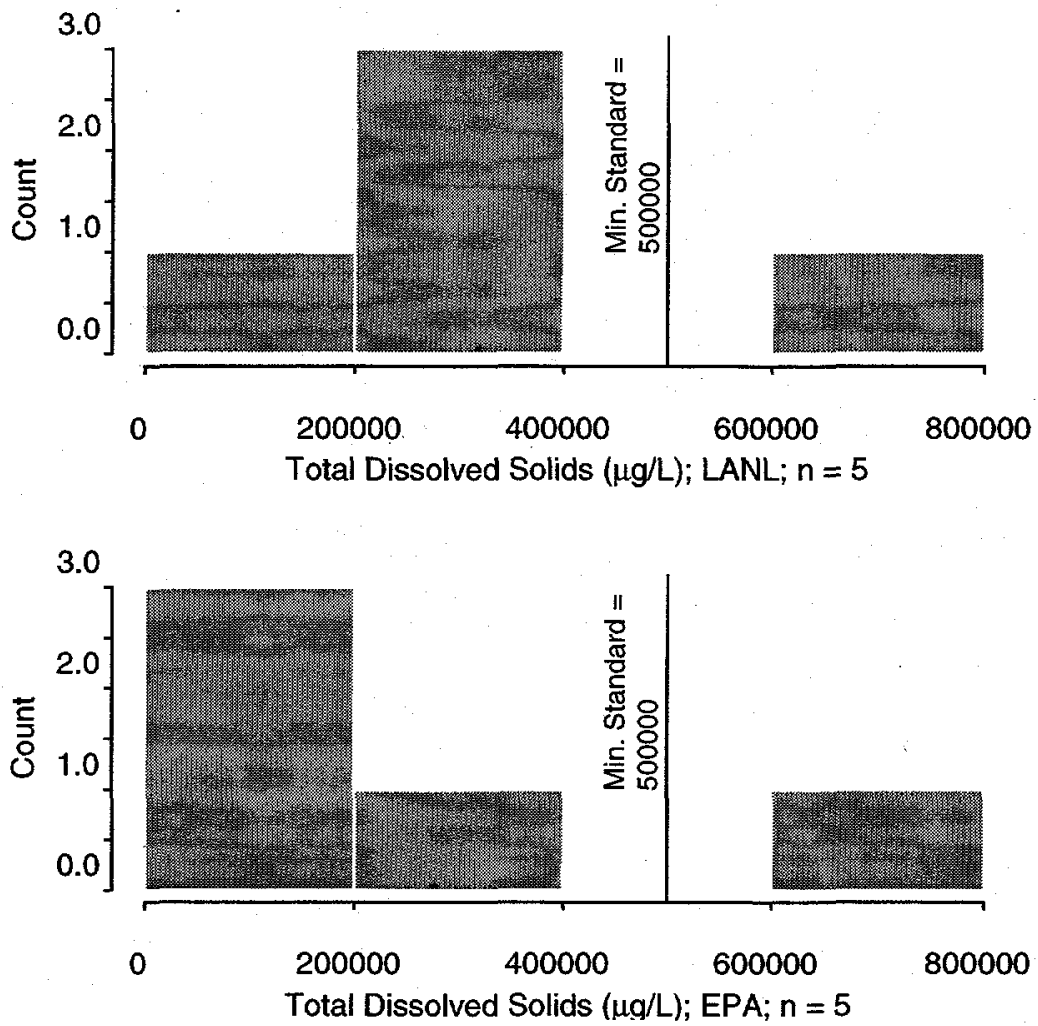

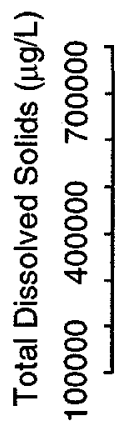

$\circ$

$\circ$

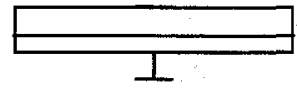

LANL

EPA 
Comparison of Total Suspended Solids ( $\mu \mathrm{g} / \mathrm{L})$ : LANL vs. EPA
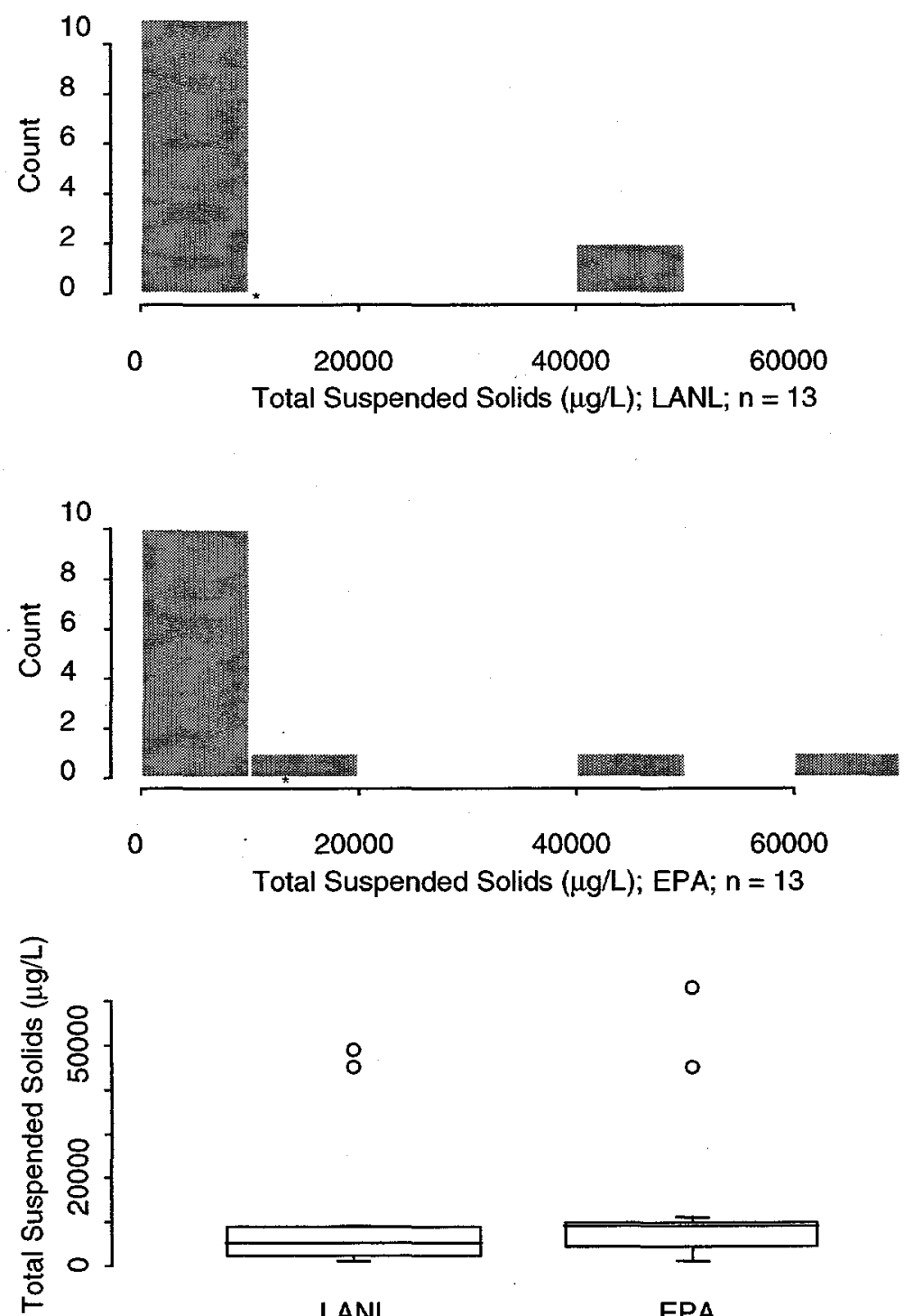

0

8

용

$0]$

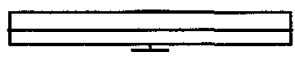

LANL

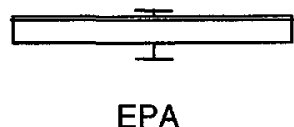


Comparison of Filtered Aluminum ( $\mu \mathrm{g} / \mathrm{L})$ : LANL vs. EPA
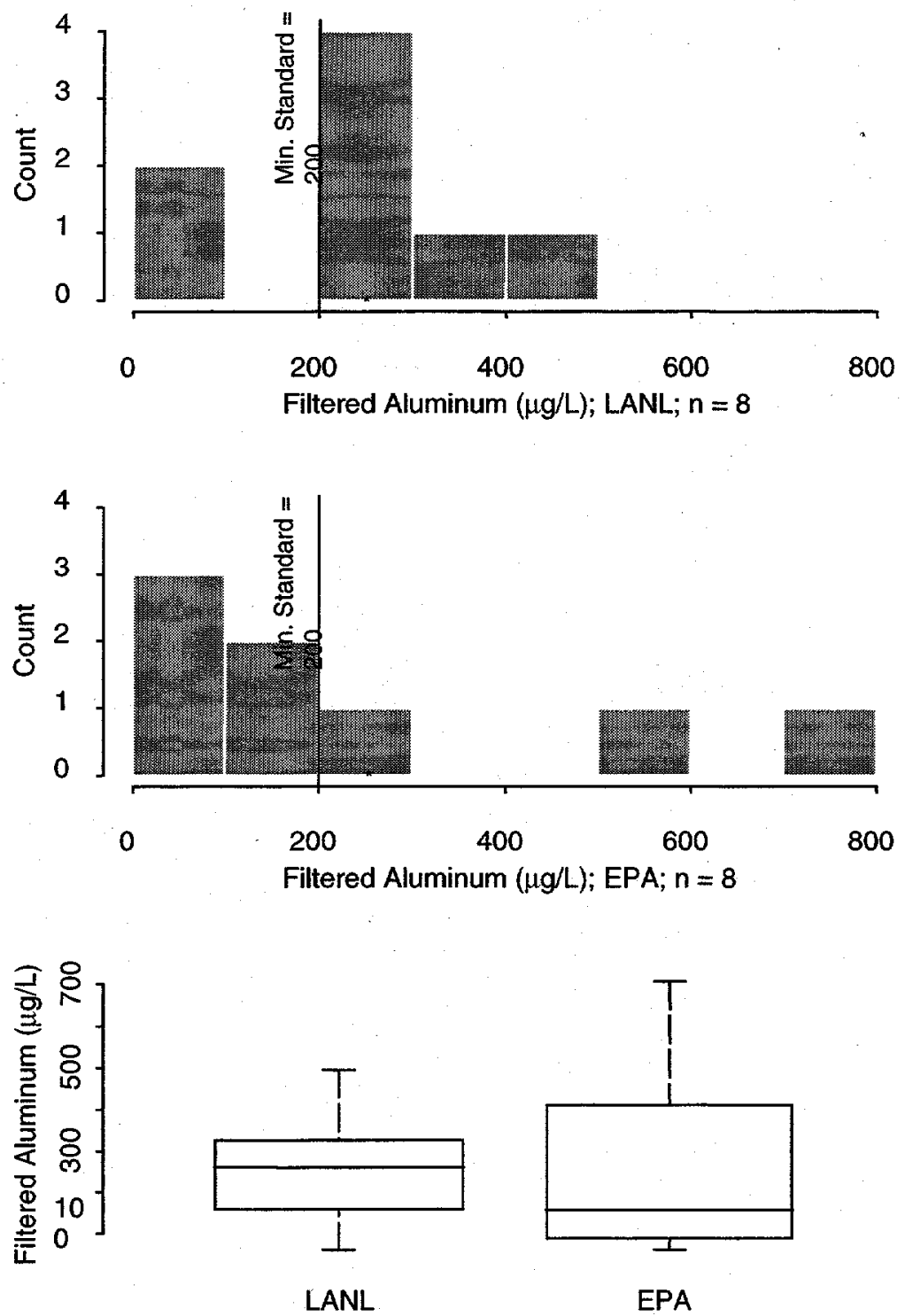
Comparison of Filtered Boron ( $\mu \mathrm{g} / \mathrm{L})$ : LANL vs. EPA
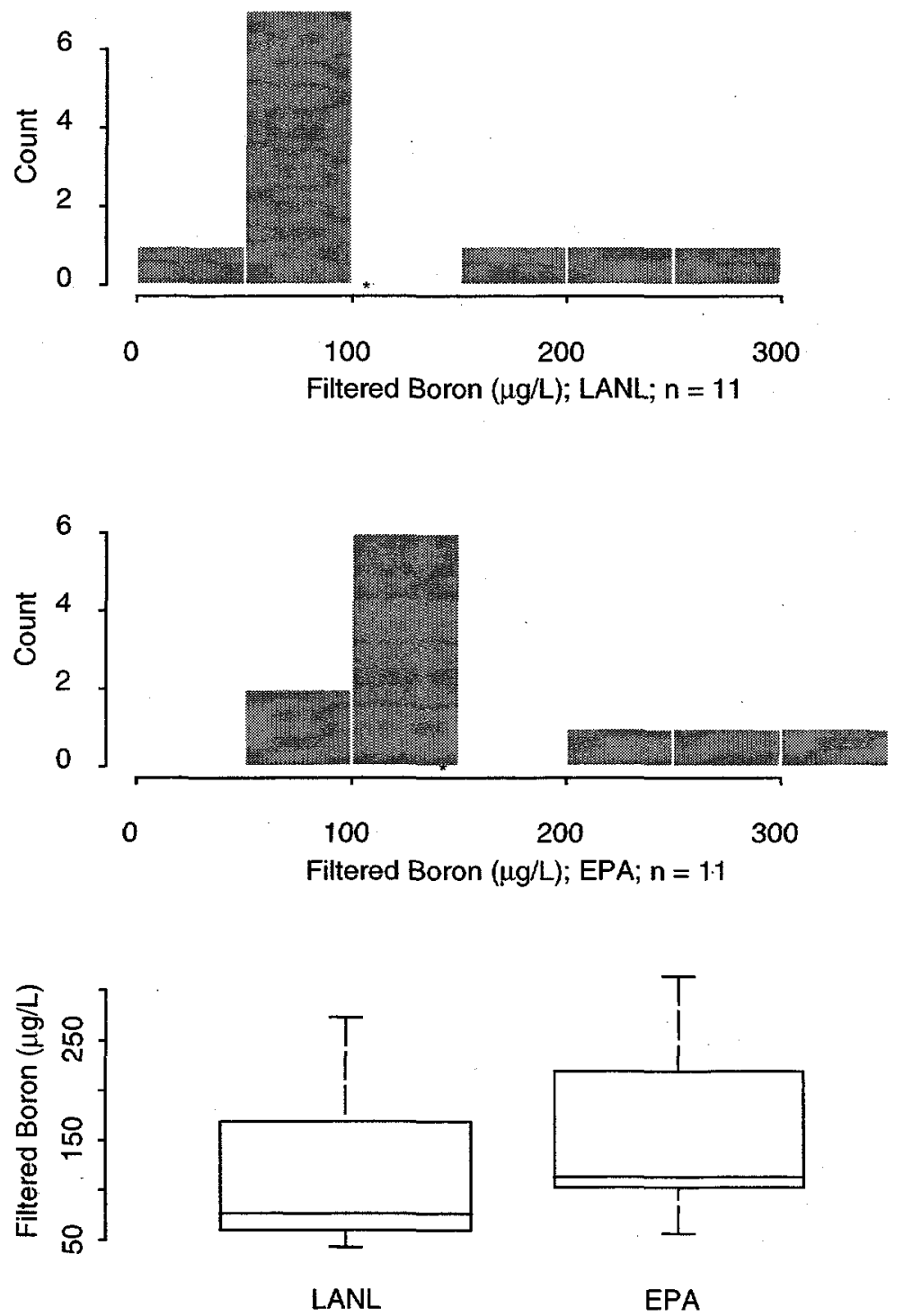
Comparison of Filtered Barium ( $\mu \mathrm{g} / \mathrm{L})$ : LANL vs. EPA
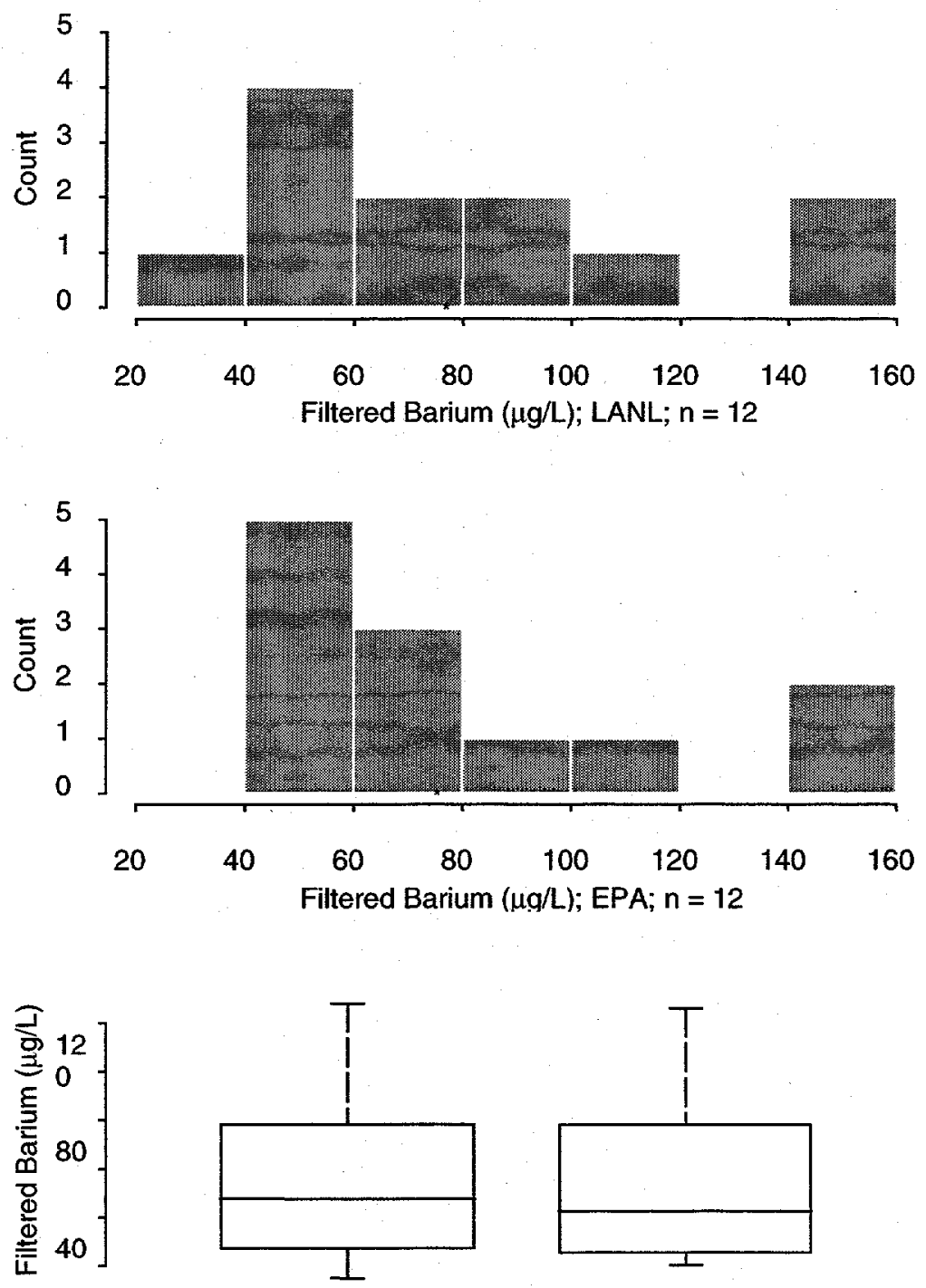

LANL

EPA 
Comparison of Filtered Iron ( $\mu \mathrm{g} / \mathrm{L})$ : LANL vs. EPA
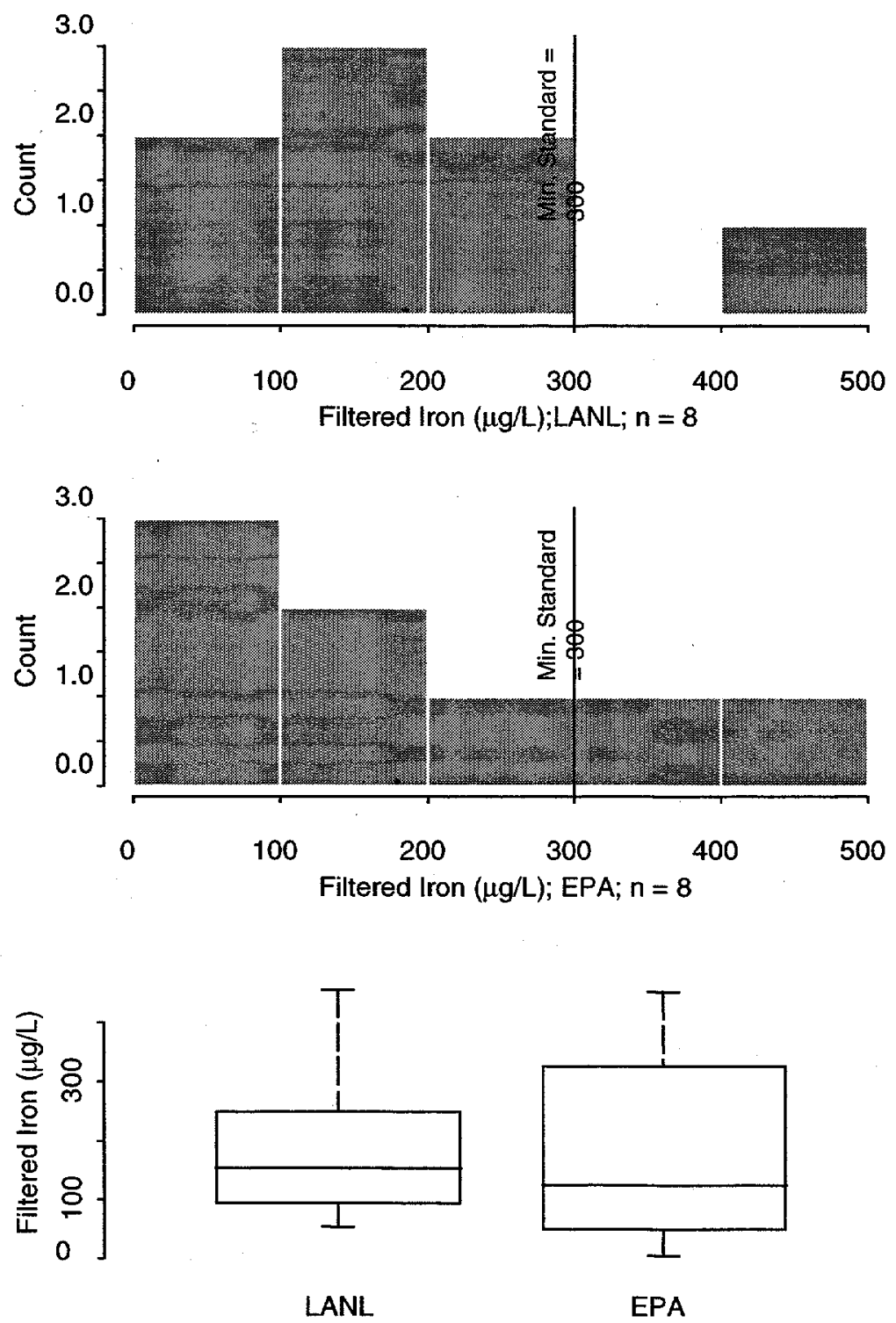
Comparison of Filtered Manganese ( $\mu \mathrm{g} / \mathrm{L})$ : LANL vs. EPA
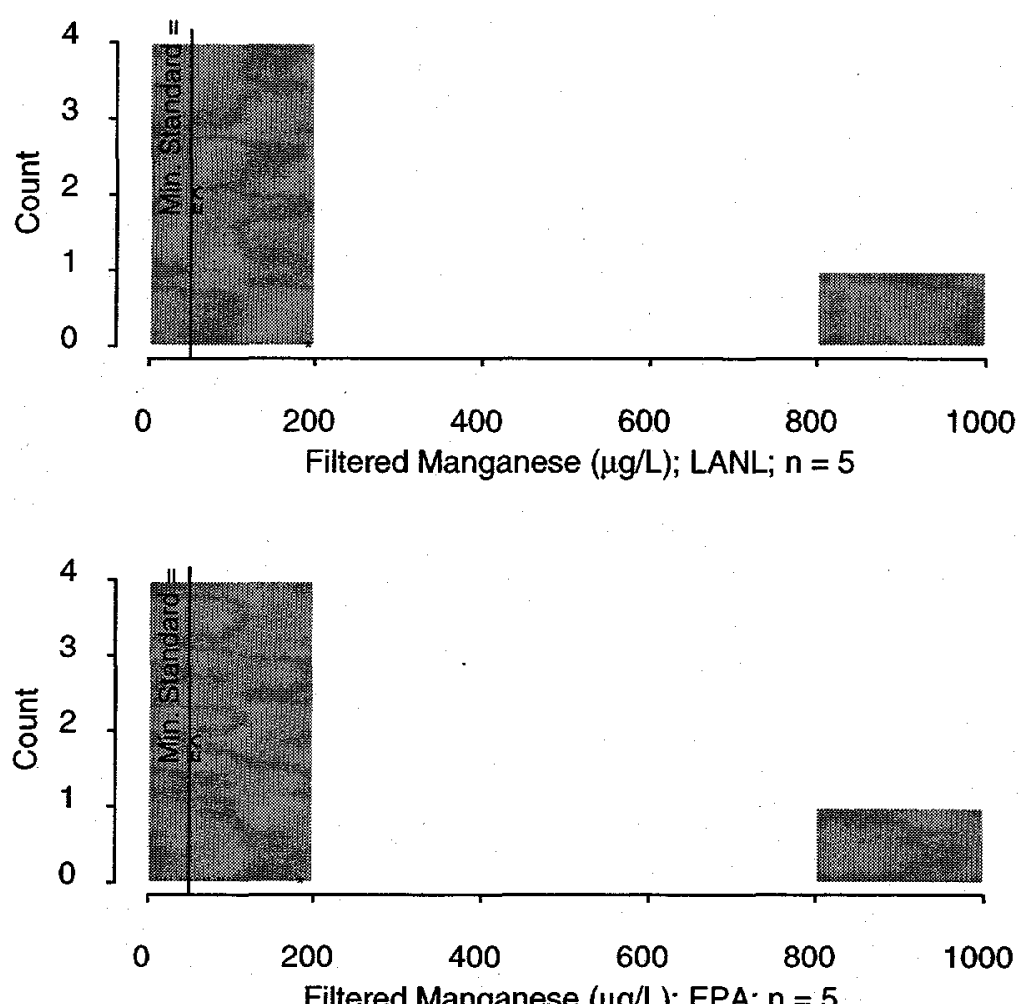

Filtered Manganese $(\mu \mathrm{g} / \mathrm{L}) ; \mathrm{EPA} ; \mathrm{n}=5$

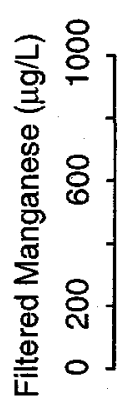

0

$\circ$

LANL

EPA 
Comparison of Filtered Molybdenum ( $\mu \mathrm{g} / \mathrm{L}$ ): LANL vs. EPA
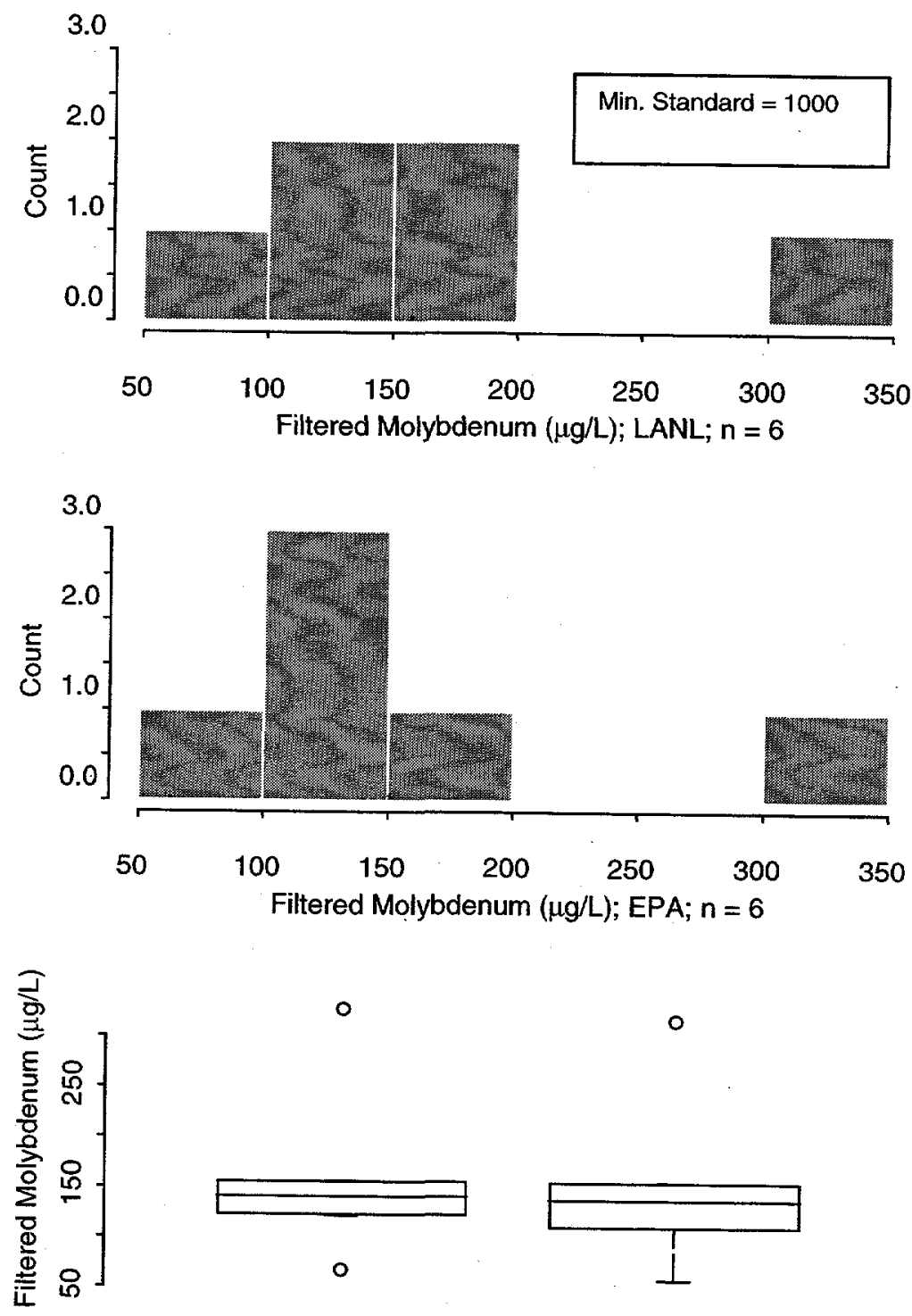

$\circ$

$\circ$

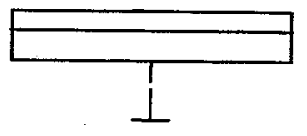

LANL

EPA 
Comparison of Unfiltered Aluminum ( $\mu \mathrm{g} / \mathrm{L})$ : LANL vs. EPA
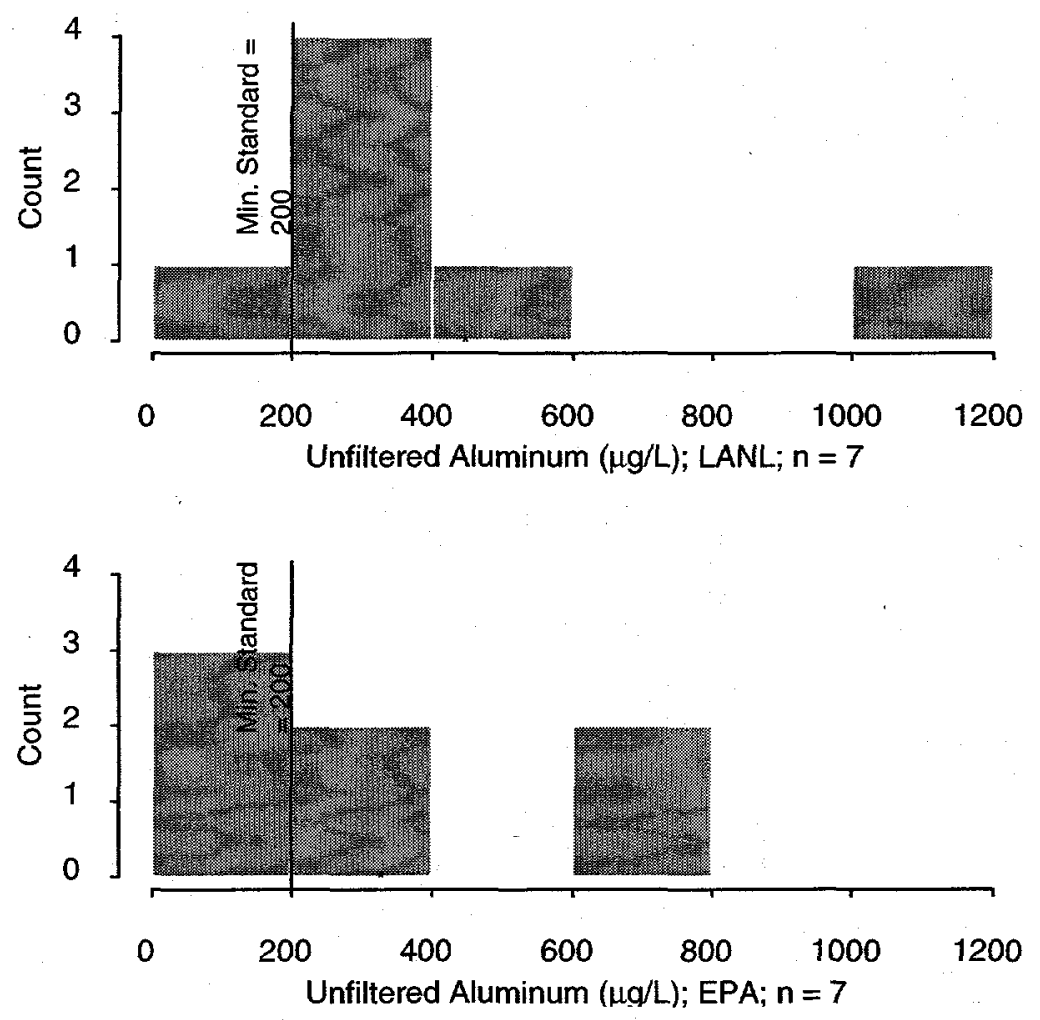

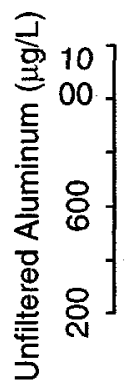

o

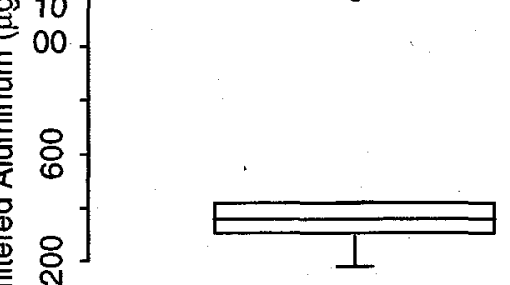

LANL

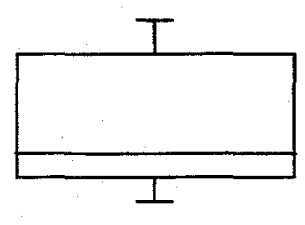

EPA 
Comparison of Unfiltered Boron $(\mu \mathrm{g} / \mathrm{L})$ : LANL vs. EPA
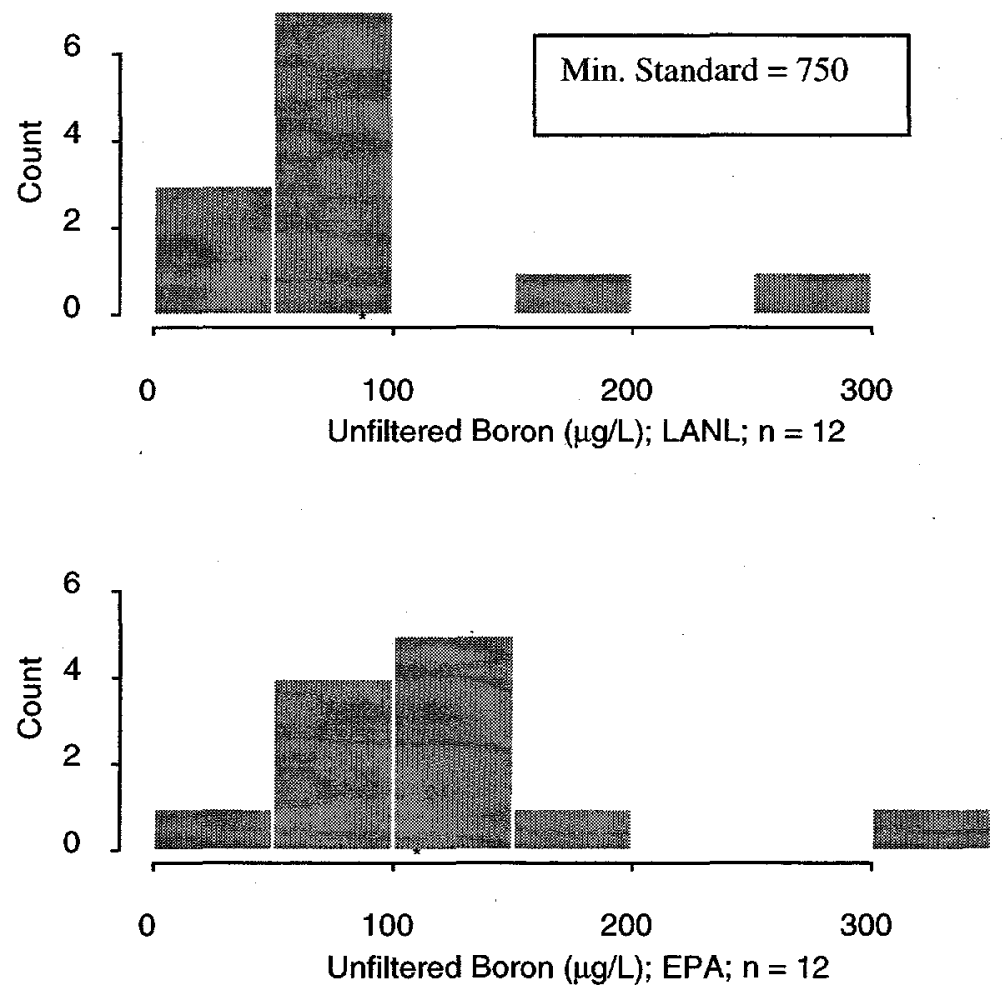

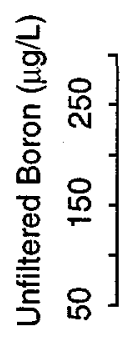

0

$\circ$

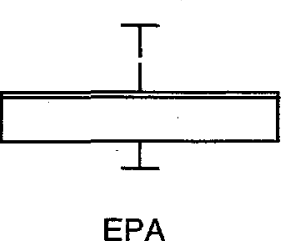


Comparison of Unfiltered Barium ( $\mu \mathrm{g} / \mathrm{L})$ : LANL vs. EPA
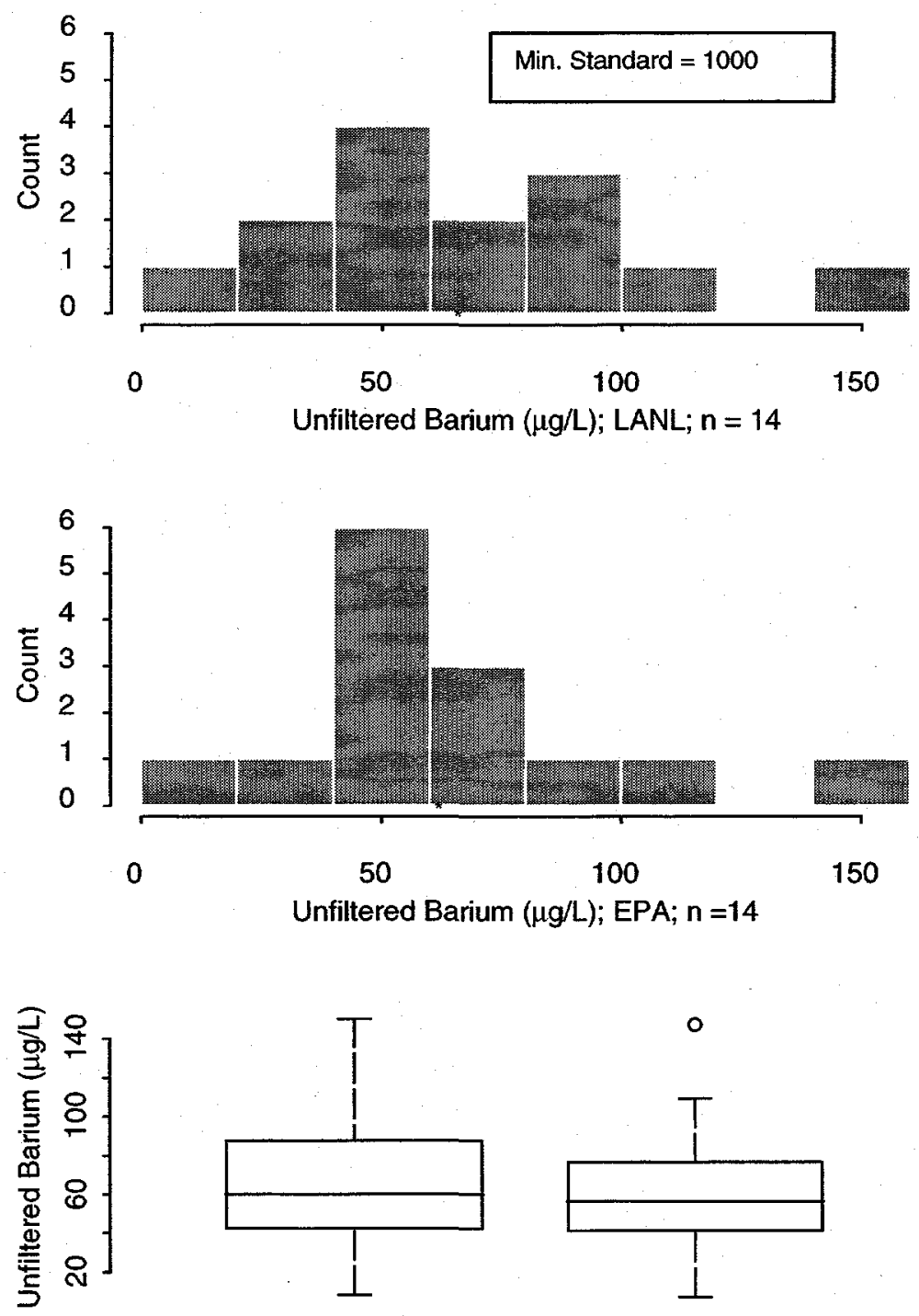

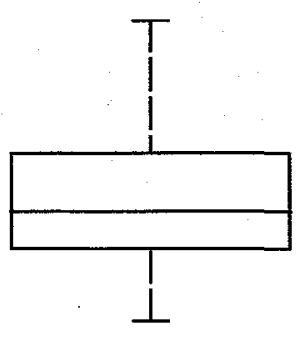

LANL

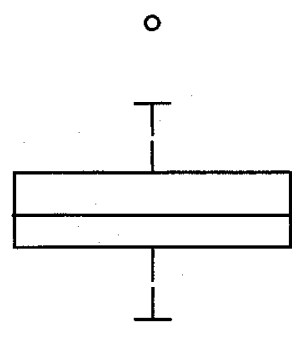

EPA 
Comparison of Unfiltered Iron ( $\mu \mathrm{g} / \mathrm{L})$ : LANL vs. EPA
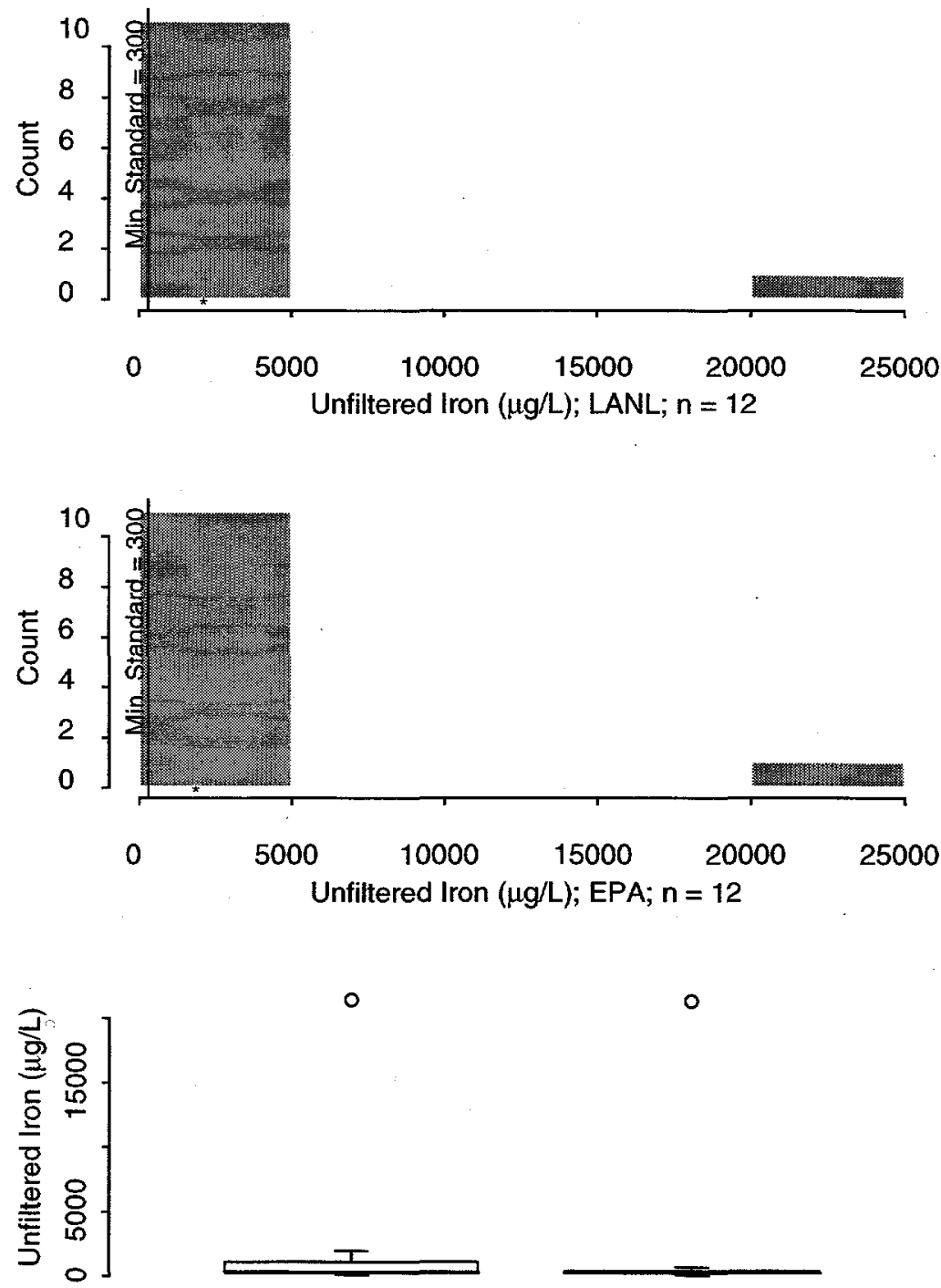

0

$\circ$
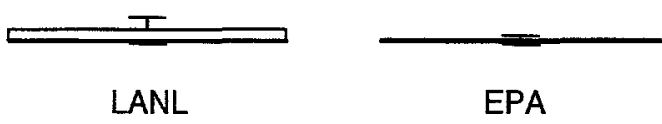
Comparison of Unfiltered Manganese ( $\mu \mathrm{g} / \mathrm{L})$ : LANL vs. EPA
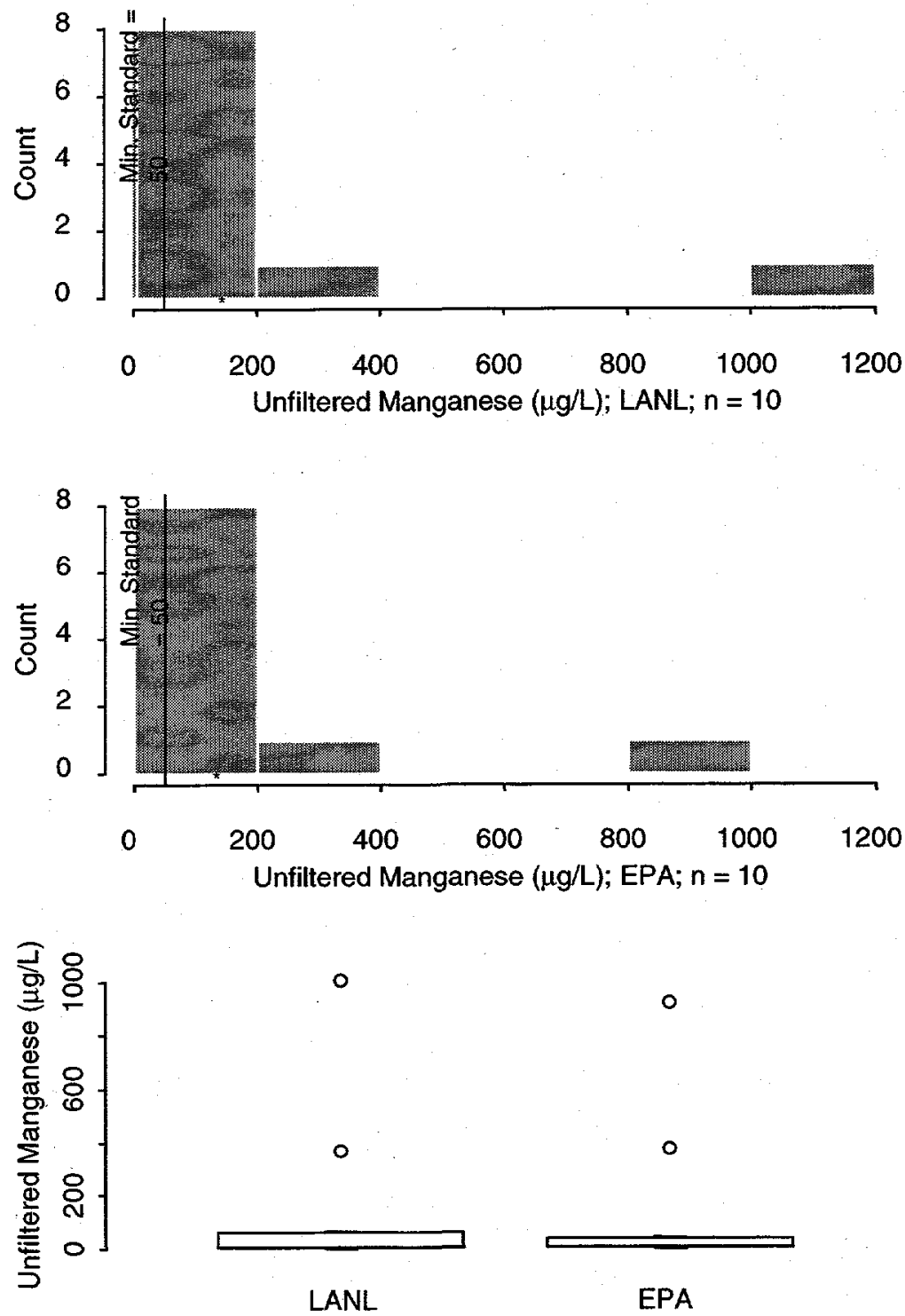
Comparison of Unfiltered Molybdenum ( $\mu \mathrm{g} / \mathrm{L}$ ): LANL vs. EPA
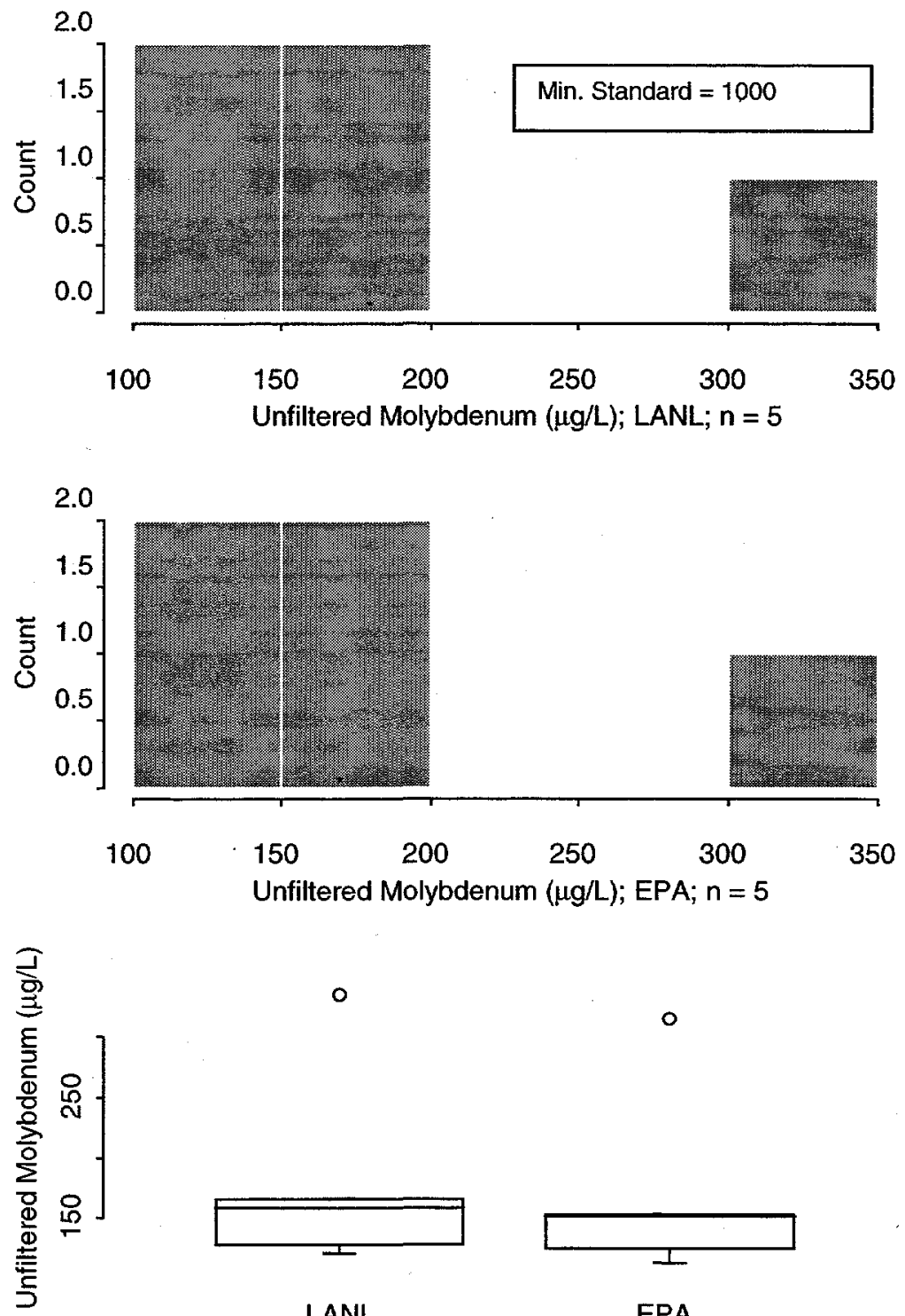

$\circ$

$\circ$

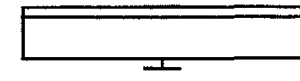

LANL

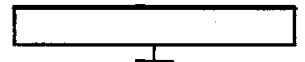

EPA 
Comparison of Gross Alpha (pCi/L): LANL vs. EPA
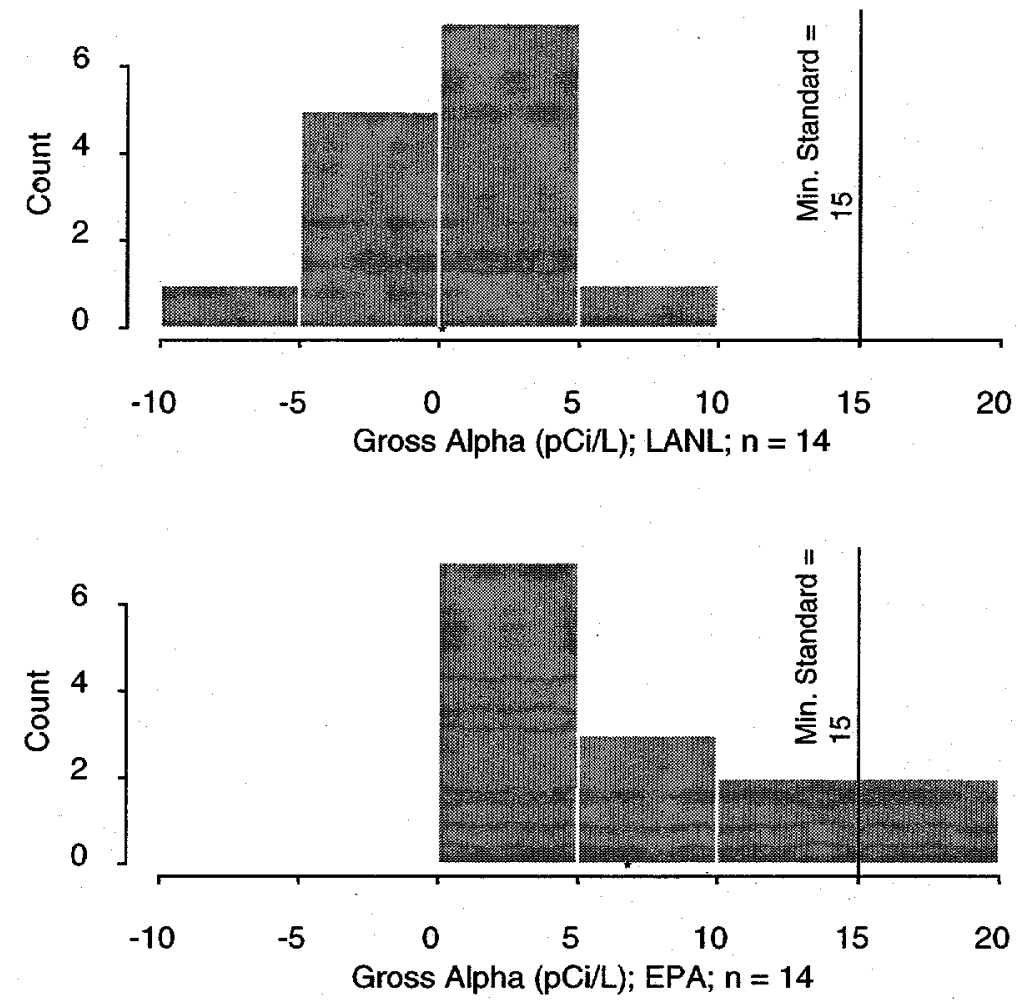

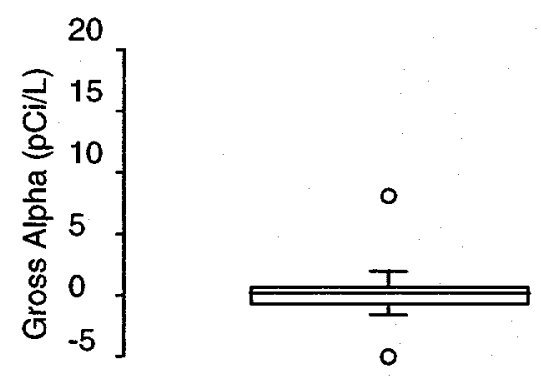

LANL

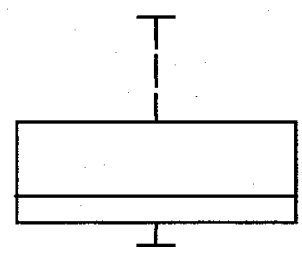

EPA 
Comparison of Gross Beta ( $\mathrm{pCi} / \mathrm{L})$ : LANL vs. EPA
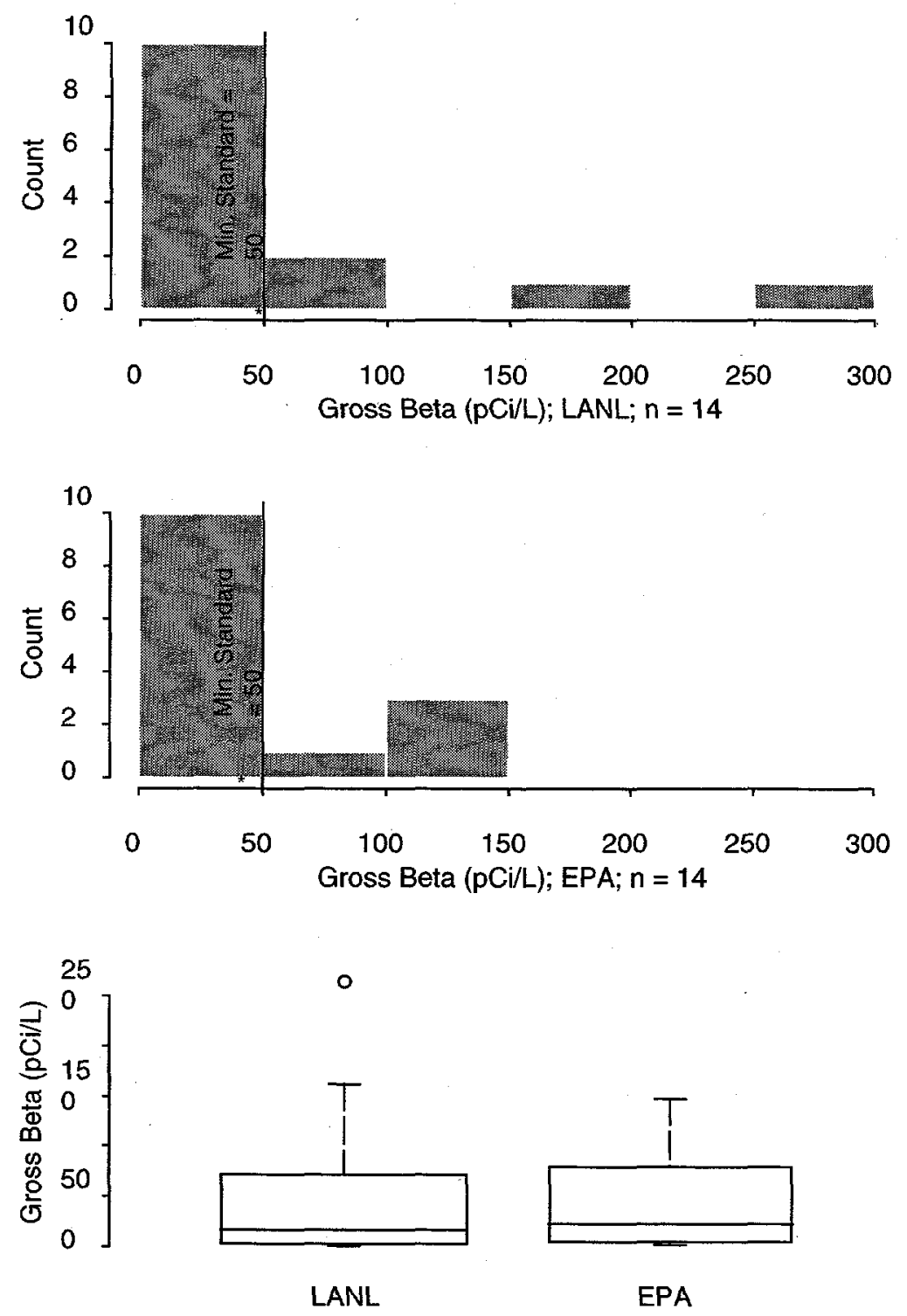
Comparison of Tritium ( $\mathrm{pCi} / \mathrm{L}$ ): LANL vs. EPA
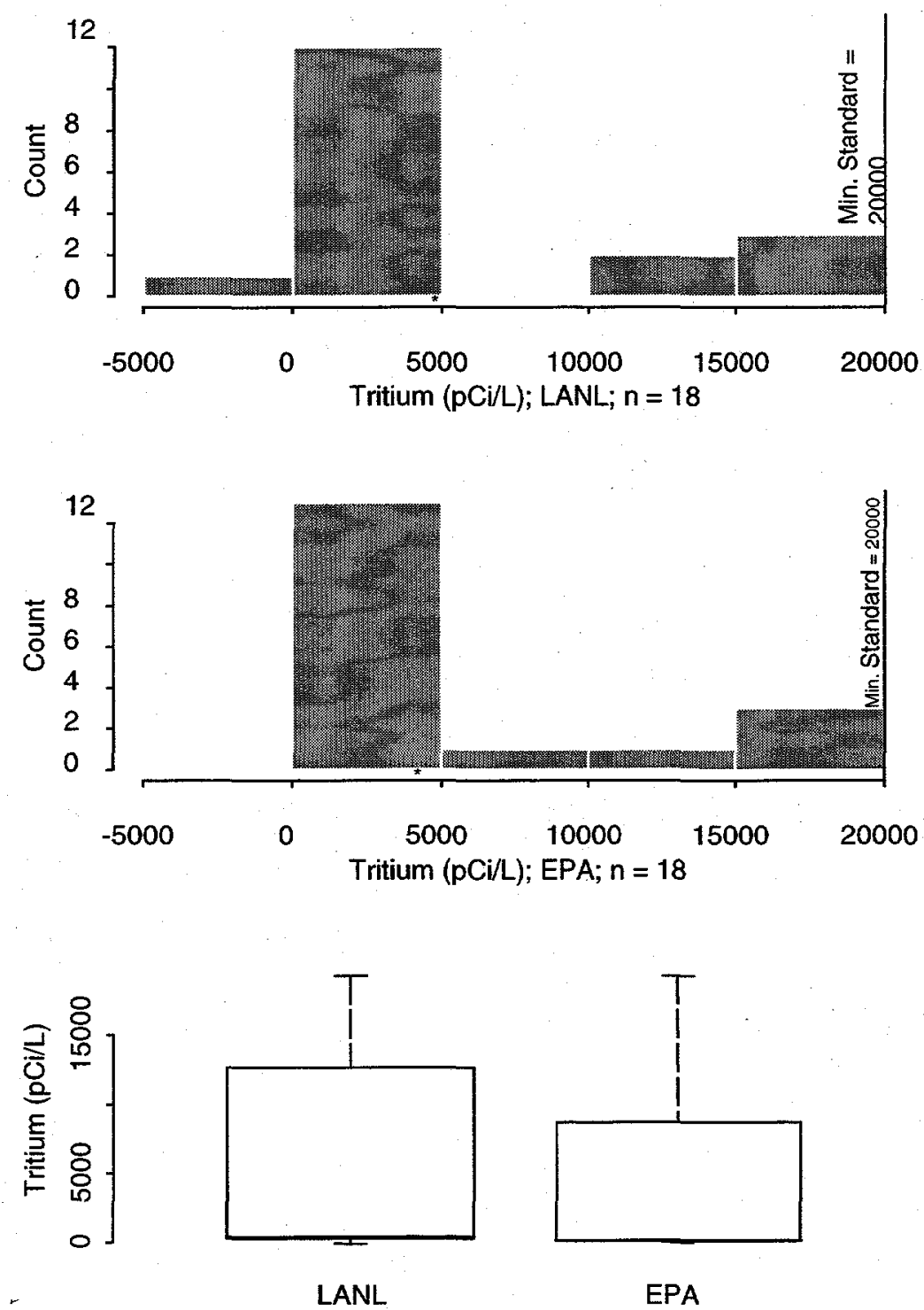
Comparison of $\mathrm{Sr}-90$ (pCi/L): LANL vs. EPA
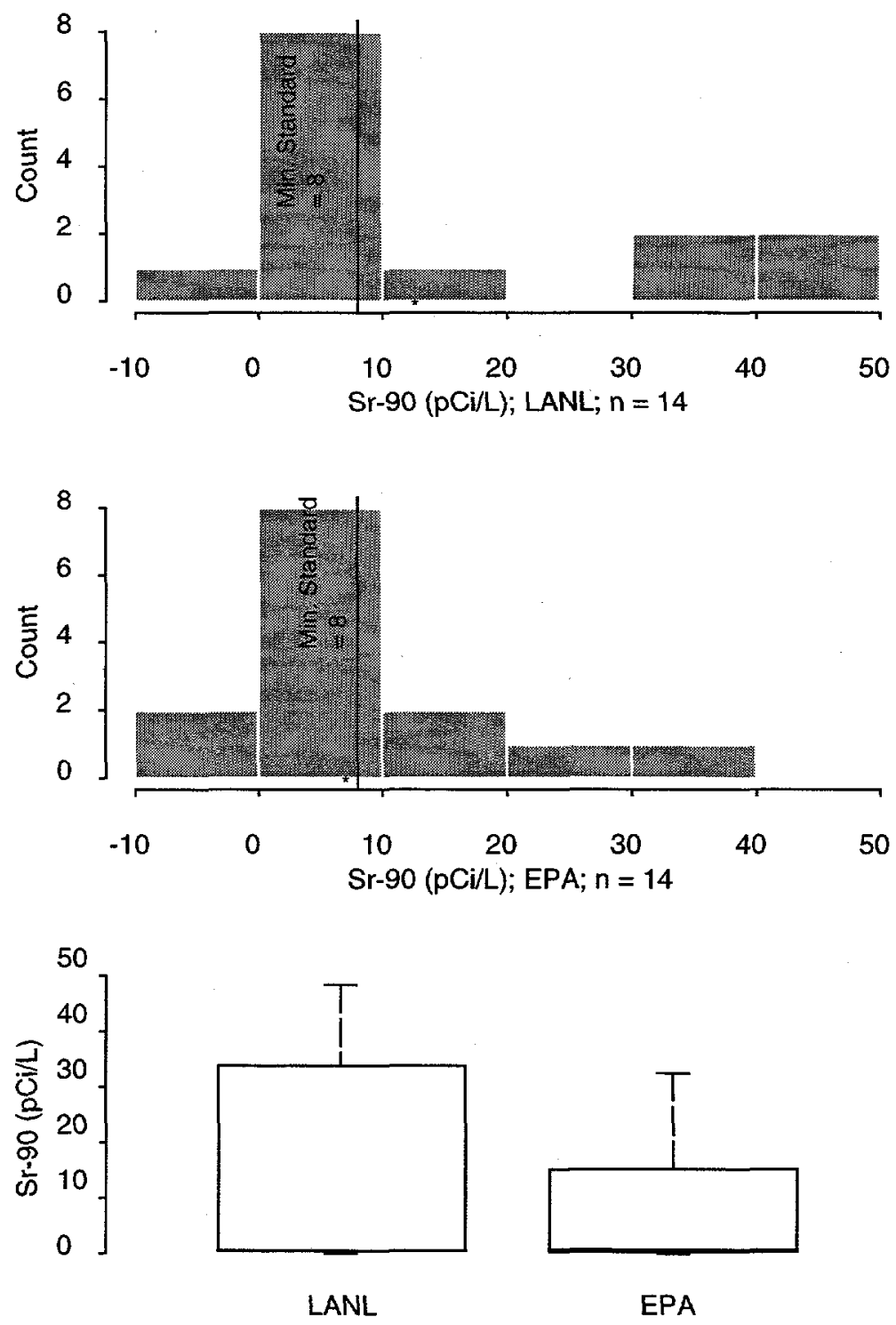